\title{
Grafos de Lyapunov, desigualdades de Poincaré-Hopf e de Morse ${ }^{l}$
}

\author{
Maria Alice Bertolim \\ Orientadora: Profa. Dra. Ketty Abaroa de Rezende. \\ Co-orientador: Prof. Dr. Oziride Manzoli Neto
}

Tese aprescntada ao Instituto de Ciências Matemáticas c de Computação - ICMC-USP, como parte dos requisitos para obtenção do título de Doutor em Ciências - Matemática.

\section{USP - São Cartos}

Outubro de 2002

\footnotetext{
${ }^{1}$ Fiste trabalho teve suporte financeiro da FAPLSP, processo n $n^{n}$ 98/13434-7

P'alavras chaves: (iratos de Lyapunow, fluxos do tipo gradiente, Continuação, desigualdades de Poincaré-l lopt.

Desigualdades de Morse

F-mail: bertolim $a$ icme.sc.usp. br
} 
A Comissão Julgadora:

Profa. Dra. Kelly Abaroa de Rezende

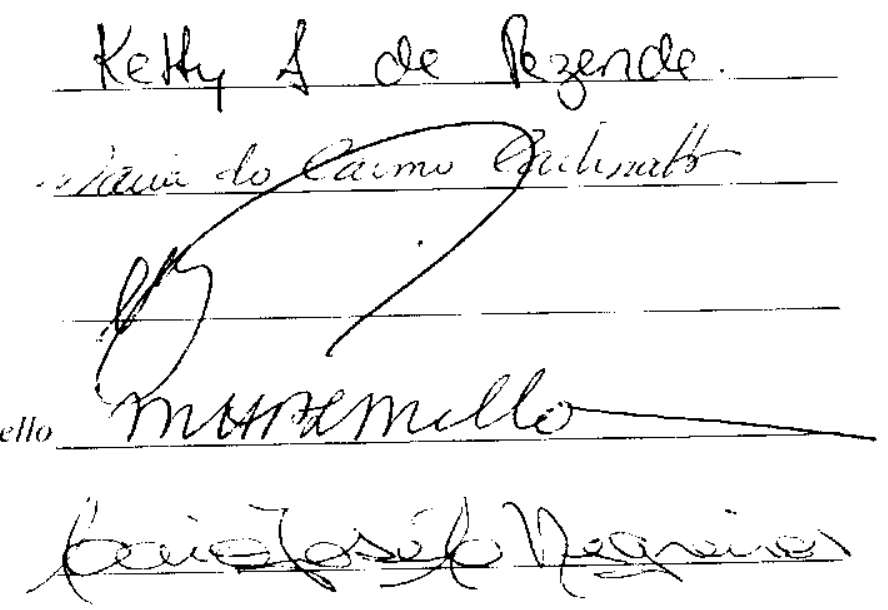

Prof. Dr. Marco Antonio Teixeira

Prola. Dra. Maria do (armo (arbinatite

Prof. Dr. Caio Jose C olletti Negreiros

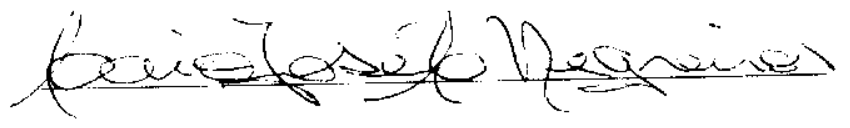


$\checkmark$ Aos meus pais Casemiro e Santina $\odot$ 
"Entrega teu caminho ao Senhor, confia Nele e o mais Ele fará" Sl 36

Viver, e não ter a vergonha de ser feliz.

Cantar e cantar e cantar

A beleza de ser um cterno aprendiz...

(Gonzaguinha) 


\section{Agradecimentos}

Existem pessoas que são importantes pelo simples fato de existirem. Elas são capazes de nos ajudar com uma simples palavra, um sorriso ou até mesmo com um olhar. Foram muitas as pessoas que, de alguma forma, me ajudaram na realização deste trabalho, $e$ tentarei registrar nestas linhas o meu agradecimento. São elas:

Ketty A. de Rezende, não só pela orientação, mas também por toda amizade e acima de tudo pela confiança depositada em mim em todos os aspectos, e a toda a sua familia, cm especial a Pedro $J$. de Rezende, que sempre esteve presente me auxiliando na parte técnica, e a Djairo e Maruja que me acolheram em sua própria casa.

Oziride Manzoli Neto, por todo carinho e incentivo para que eu continuasse meus estudos.

Aos meus pais Casemiro e Santina, meus irmãos Edson e Wilson, minhas cunhadas Shirlei e Mirian, minhas sobrinhas Mariana, Ana Alice e 'aquele' que há de vir com muita saúde. Obrigada por todo carinho, incentivo e por sempre terem compreendido a minha ausência. Amo vocês!

$A$ todos os professores e funcionários de:

- E.E.P.S.G. "Ferdinando Ienny" de Ouro Verde, onde iniciei os meus estudos;

- FCT-UNESP de Presidente Prudente, que me incentivaram a continuar os meus estudos;

- ICMC-USP de São Carlos, em especial Farid, Maria do Carmo e Ires;

- IMECC-UNICAMP, em especial a Profa. Margarida P. Mello pela importante participação na realização deste trabalho;

- Northwestern University, em especial ao Prof. John Franks por toda atenção e a Marco por todo o carinho.

Aos amigos:

- "de guerra": Vera, Luciana, José, Márcio e Eliane, presentes desde o meu mestrado;

- "de música": Peter, Thelma, Fabiano, Ezequiel, Daniela, Gian e Lucas, vocês são especiais!

- "de noite": Daniela, Cláudia, Ana Paula e Marcinha, vou sentir saudades dos nossos passeios!

- "anjo da guarda do lab": Sadao;

- "engenheiras de Campinas" Rúbia, Tatiane e Aline;

- Andréa, Luciene, Liane, Humberto, Auri, Cecilia, Ana Lúcia e a todos os meus amigos.

$A$ você, por talvez ter apenas sorrido para mim e tornado meus dias mais bonitos.

Agradeço enfim a Deus não somente pelo dom da vida, mas também por ter me orientado durante todo este trabalho e por ter me dado a oportunidade de conviver com todas as pessoas acima citadas.

Obrigada! 


\section{Resumo}

Grafos de Lyapunov carregam informações dinâmicas de fluxos do tipo gradiente bem como informaçōes topológicas do espaço de fase correspondente, o qual tomamos como uma variedade orientável fechada de dimensão $n$. Neste trabalho, os grafos de Lyapunov $L\left(h_{0}, \ldots, h_{n}, \kappa\right)$ considerados podem representar fluxos suaves em variedades orientáveis fechadas de dimensão maior ou igual a dois, com cycle number $\kappa$. Mostramos que as desigualdades de Poincaré-Hopf são condições necessárias e suficientes para um grafo abstrato de Lyapunov $L\left(h_{0}, \ldots, h_{n}, \kappa\right)$ ser continuado a um grafo abstrato de Lyapunov do tipo Morse com cycle rank maior ou igual a $\kappa$. A continuação, que é apresentada por meio de um algoritmo, é mostrada ser única em dimensões dois e três. Em outras dimensões, apresentamos números exatos de possíveis continuações de $L\left(h_{0}, \ldots, h_{n}, \kappa\right)$. Mostramos também que um grafo abstrato de Lyapunov em dimensão maior ou igual a dois, com cycle number $\kappa$, satisfaz as desigualdades de Poincaré-Hopf se, e somente se, satisfaz as desigualdades de Morse e o primeiro número de Betti $\gamma_{1}$ é pelo menos $\kappa$. Definimos o politopo de Morse, $P_{\kappa}\left(h_{0}, \ldots, h_{n}\right)$, como sendo o casco convexo da coleção de todos os vetores de números de Betti obtidos das desigualdades de Morse e da desigualdade $\gamma_{1} \geq \kappa$ para dados pré-fixados $\left(h_{0}, \ldots, h_{n}, \kappa\right)$. Finalmente, associamos um politopo de Morse a uma família de grafos de Lyapunov $\mathcal{L}\left(h_{0}, \ldots, h_{n}, \kappa\right)$ e estabelecemos propriedades geométricas deste politopo. 


\begin{abstract}
Lyapunov graphs carry dynamical information of gradient-like flows as well as topological information of its phase space which is taken to be a closed orientable $n$-manifold. In this thesis the Lyapunov graphs $L\left(h_{0}, \ldots, h_{n}, \kappa\right)$ considered may represent smooth flows on closed orientable $n$-manifolds, $n \geq 2$, with cycle number $\kappa$. We will show that the Poincarê-Hopf inequalitics are necessary and sufficient conditions for an abstract Lyapunov graph $L\left(h_{0}, \ldots, h_{n}, \kappa\right)$ to be continued to an abstract Lyapunov graph of Morse type with cycle rank greater or equal to $\kappa$. The continuation which is presented by means of a constructive algorithm, is shown to be unique in dimensions two and threc. In all other dimensions, the exact number of possible continuations of $L$ are presented. We show that an abstract Lyapunov graph $L\left(h_{0}, \ldots, h_{n}, \kappa\right)$ in dimension $n$ greater than or equal to two, with cycle number $\kappa$, satisfies the Poincaré-Hopf inequalities if and only if it satisfies the Morse inequalities and the first Betti number $\gamma_{1}$ is at least equal to $\kappa$. The convex hull of the collection of all Betti number vectors which satisfy the Morse inequalities and the inequality $\gamma_{1} \geq \kappa$ for a pre-assigned data determines a Morse polytope $\mathcal{P}_{\kappa}\left(h_{0}, \ldots, h_{n}\right)$. Finally, we associate a Morse polytope, $\mathcal{P}_{\kappa}\left(h_{0}, \ldots, h_{n}\right)$ to a family of Lyapunov graphs $\mathcal{L}\left(h_{0}, \ldots, h_{n}, \kappa\right)$ and determine geometrical properties of this polytope.
\end{abstract}




\section{Índice}

$\begin{array}{ll}\text { Introdução } & 1\end{array}$

1 Preliminares $\quad 7$

1.1 Fluxos do tipo gradiente $\ldots \ldots \ldots \ldots \ldots$

1.2 Grafos de Lyapunov $\ldots \ldots \ldots \ldots \ldots$

1.3 Teoria de Conley . . . . . . . . . . . . . . . . 11

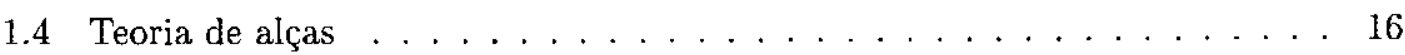

1.5 Explosões e implosões de vértices . . . . . . . . . . . . . . . . . 22

2 Desigualdades de Poincaré-Hopf 25

2.1 Desigualdades de Morse . . . . . . . . . . . . . . . . . . 25

2.2 Filtração do grafo . . . . . . . . . . . . . . . . . . . 27

2.3 Desigualdades de Poincarć-Hopf . . . . . . . . . . . . . . . . . 29

2.3 .1 Dualidade do índice de Conlcy . . . . . . . . . . . . . . . 29

2.3.2 Scqüência longa exata de um par índice . . . . . . . . . . 30

2.3.3 Caso de dimensão ímpar, $n=2 i+1 \ldots \ldots 31$

2.3.4 Caso de dimensão par, $n=2 i \ldots \ldots \ldots \ldots$

2.4 Desigualdades de Poincaré-Hopf para variedades fechadas . . . . . . . . . 37

3 Continuação de Grafos de Lyapunov 39

3.1 Algoritmo da Explosão . . . . . . . . . . . . . . . . . . . . . . 40

3.1.1 Explosão Parcial de um vértice . . . . . . . . . . . . . . . 40

3.1.2 Algoritmo da explosão de um vértice do tipo sela . . . . . . . . . 44

3.1.3 Algoritmo de Explosão para $n=2 \ldots \ldots \ldots 3$ 
iv.

3.2 Soluções para os Sistemas Lineares . . . . . . . . . . . . . . . . . . 54

3.2 .1 Caso $n$ ímpar . . . . . . . . . . . . . . . . . . . . 54

$3.2 .2 \quad$ Caso $n=0 \bmod 4 \ldots \ldots \ldots \ldots \ldots$

$3.2 .3 \quad$ Caso $n=2 \bmod 4 \ldots \ldots \ldots \ldots$

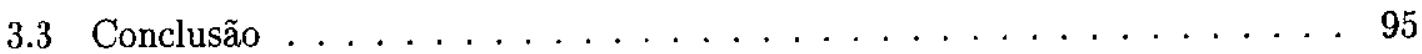

4 Desigualdades de Poincaré-Hopf e Desigualdades de Morse 99

4.1 Resultados de equivalência . . . . . . . . . . . . . . . 100

4.1 .1 Caso n ímpar . . . . . . . . . . . . . . . . . . . . . 101

$4.1 .2 \quad$ Caso $\mathrm{n}=\mathbf{0} \bmod 4 \ldots \ldots \ldots \ldots \ldots$

$4.1 .3 \quad$ Caso $n=2 \bmod 4 \ldots \ldots \ldots \ldots$

4.2 Politopos . . . . . . . . . . . . . . . . . . . . . . . 121

4.2 .1 Caso $n$ ímpar . . . . . . . . . . . . . . . . . . . . . 122

$4.2 .2 \quad$ Caso $\mathrm{n}=0 \bmod 4 \ldots \ldots \ldots \ldots$

$4.2 .3 \quad$ Caso $n=2 \bmod 4 \ldots \ldots \ldots \ldots \ldots \ldots$

5 Grafos de Lyapunov, Desigualdades de Poincaré-Hopf e Desigualdades

$\begin{array}{ll}\text { de Morse } & 137\end{array}$

5.1 Resultados de Continuação . . . . . . . . . . . . . . . . . . . . 139

5.2 Resultados sobre as desigualdades de Morse . . . . . . . . . . . 150

5.3 Politopo de Morse . . . . . . . . . . . . . . . . . . . . . . . 159

5.3 .1 Caso $n$ ímpar . . . . . . . . . . . . . . . . . . . 160

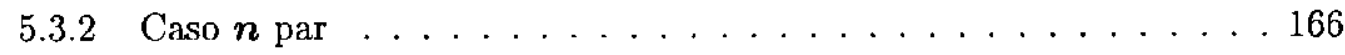

$\begin{array}{ll}\text { Lista de figuras } & 171\end{array}$

$\begin{array}{lc}\text { Tabela de símbolos } & 173\end{array}$

$\begin{array}{ll}\text { Referências Bibliográficas } & 175\end{array}$

$\begin{array}{ll}\text { Índice Remissivo } & 178\end{array}$ 


\section{Introdução}

Grafos de Lyapunov foram inicialmente introduzidos por Franks cm [12]. Usamos estes grafos como um organizador de informações homológicas locais e globais do fluxo e do espaço de fase $M$, uma variedade orientável fechada de dimensão $n$.

Dado um fluxo contínuo $\phi_{t}: M \rightarrow M$, resultados de Conley [5] implicam na existência de uma função contínua de Lyapunov $f: M \rightarrow \mathbb{R}$ associada ao fluxo com a propriedade que $f$ decresce ao longo das órbitas fora do conjunto recorrente por cadeia, isto é, se $x \notin R$ então $f\left(\phi_{t}(x)\right)<f\left(\phi_{s}(x)\right)$ para $t>s$ e é constante sobre as componentes do conjunto recorrente por cadeia $R$.

Definimos a relação de equivalência sobre $M$ onde $x \sim \rho$ se, e somente se, $x$ e $y$ pertencem a mesma componente conexa de um conjunto de nível de $f$. Chamamos $M / \sim_{f}$ um grafo de Lyapunov. Cada vértice $v_{k}$ representa uma componente $R_{k}$ do conjunto recorrente por cadeia $R$ ou, mais geralmente, um conjunto de Morse e logo pode ser rotulado com invariantes dinâmicos. Cada aresta representa um conjunto de nível cartesiano um intervalo e logo pode ser rotulada com invariantes topológicos do conjunto de nível.

Um grafo abstrato de Lyapunov em dimensão $n$ é um grafo orientado, finito e conexo, sem ciclos orientados, e com rótulos nos seus vértices e arestas. Cada vértice pode ser rotulado com um fluxo recorrente por cadeia sobre um espaço compacto de dimensão $n$ ou com algum invariante dinâmico deste fluxo. Cada aresta pode ser rotulada com invariantes topológicos de uma variedade fechada de dimensão $(n-1)$. Neste trabalho, rotulamos o vértice $v_{k}$ de um grafo abstrato de Lyapunov com as dimensões dos índices homológicos de Conley de uma componente do conjunto recorrente por cadeia, $R_{k}, \operatorname{dim} C H_{j}\left(R_{k}\right)=h_{j}\left(v_{k}\right)$, com $j=0, \ldots n$. Logo, cada vértice é rotulado com uma lista de inteiros não negativos $\left(h_{0}\left(v_{k}\right), \ldots, h_{n}\left(v_{k}\right), \kappa\left(v_{k}\right)\right)$, onde $\kappa\left(v_{k}\right)$ é o cycle number do vértice $v_{k}$. Uma notação 
alternativa é rotular o vértice com $h_{j}\left(v_{k}\right)=n_{j}$ sempre que $n_{j} \neq 0$ e $\kappa\left(v_{j}\right)=k_{j}$ sempre que $k_{j} \neq 0$. A última notação é mais conveniente sempre que $\left(h_{0}\left(v_{k}\right), \ldots, h_{n}\left(v_{k}\right), \kappa\left(v_{k}\right)\right)$ tenha muitas entradas iguais a zero. Também, por simplicidade omitimos a referência ao vértice $v_{k}$ sempre que possível. Escolhemos rotular as arestas com os números de Betti de uma variedade fechada de dimensāo $(n-1)$ o qual agrupamos num vetor de números de Betti. Um vetor de números de Betti de dimensão né um vetor de inteiros não negativos $\operatorname{com}\left(\gamma_{0}, \gamma_{1}, \ldots, \gamma_{n-1}, \gamma_{n}\right)$ onde $\gamma_{n-k}=\gamma_{k}, \gamma_{0}=\gamma_{n}=1$ e $\gamma_{n / 2}$ é par se $n$ é par ${ }^{2}$. Denotamos por $L\left(h_{0}, \ldots, h_{n}, \kappa\right)$ um grafo abstrato de Lyapunov $L$ com conjunto de vértices $V$ e cycle rank $\kappa_{L}$, onde $h_{j}=\sum_{v_{k} \in V} h_{j}\left(v_{k}\right)$ e $\kappa_{V}=\sum_{v_{k} \in V} \kappa\left(v_{k}\right)$ e $\kappa=\kappa_{L}+\kappa_{V}$. Nos referimos a $\kappa$ como o cycle number de um grafo abstrato de Lyapunov.

Um grafo abstrato de Lyapunov do tipo Morse será definido subseqüentemente, mas basicamente é um grafo abstrato de Lyapunov com cada vértice $v$ rotulado com singularidades não degeneradas de índice de Morse $j$, i.e., $h_{j}(v)=1$ e todos os cycle numbers dos vértices iguais a zero. É fácil ver que o cycle number de um grafo abstrato de Lyapunov do tipo Morse é igual a scu cycle rank.

Certos invariantes topológicos da variedade, bem como invariantes do fluxo impoem restrições sobre o grafo de Lyapunov. Por exemplo, em [8] foi mostrado que o cycle rank ${ }^{3} \kappa$ de um grafo de Lyapunov é o limite inferior para o gênero de Cornea ${ }^{4}$ da variedade $g(M)$, que é sempre menor ou igual ao primeiro número de Betti de $M, \kappa \leq g(M) \leq \gamma_{1}(M)$. Isto generaliza um teorema de Franks [12] que afirma que se $M$ é simplesmente conexa então $\kappa=0$. Outros teoremas de caracterização de fluxos sobre variedades usando grafos de Lyapunov e alguns resultados de realização de um grafo abstrato de Lyapunov para casos particulares encontram-se cm [7], [9], [10], [11].

A motivação inicial deste trabalho foi verificar quais grafos abstratos de Lyapunov $L$ poderiam ser realizados como fluxos em variedades. Esta pergunta é demasiadamente

\footnotetext{
${ }^{2}$ Relaxamos a condição de $\gamma_{i}$ ser par no caso $2 i=0 \bmod 4$ quando consideramos vetores de números de Betti nas desigualdades de Morse

${ }^{3} \mathrm{O}$ cycle rank de um grafo é o número máximo de arestas que podem ser removidas sem desconectar o grafo.

${ }^{4}$ Este gênero é o número máximo de subvariedades mutuamente disjuntas, suaves, compactas, conexas de codimensão um (two-sided de codimensão um) que não desconectam as variedades fechadas suaves $M$. Ver [6].
} 
geral e a tratamos inicialmente do ponto de vista dinâmico da continuação de fluxos. Inspirados no trabalho de Reineck [19] que trata da continuação de fluxos a fluxos gradientes (i.e. fluxos Morse), verificamos sob quais condições, $L$, pode ser continuado a um grafo abstrato de Lyapunov do tipo Morse.

Como cada vértice deve representar uma componente do conjunto recorrente por cadeia que assumimos ser um conjunto invariante isolado dentro de um bloco isolante, é natural que tratemos em primeiro lugar a questão da continuação local. A continuação de $L$ tem que ser feita em cada vértice $v$ através da explosão de $v$. Esta explosão que será definida posteriormente, consiste em substituir $v$ por um semi-grafo abstrato de Lyapunov do tipo Morse $L_{v}$ respeitando os rótulos das arestas incidentes a $v$ e mantendo as dimensões dos índices homológicos de $v$ na soma dos índices homológicos dos vértices de $L_{v}$. Provamos que $L$ pode ser continuado a um grafo abstrato de Lyapunov do tipo Morse se e somente se os vértices satisfizerem as desigualdades de Poincaré-Hopf para blocos isolantes.

Um grafo que nāo admite uma continuação a um grafo de Lyapunov do tipo Morse não pode ser realizado como um fluxo contínuo em nenhuma variedade. Isto nos levou a investigar as condições globais que assegurariam a continuação de um grafo abstrato de Lyapunov $L$ a um grafo abstrato de Lyapunov do tipo Morse. Verificamos que $L$ admite uma continuação se e somente se satisfaz as desigualdades de Poincaré-Hopf (2.21)-(2.23). Dizemos que um grafo abstrato de Lyapunov $L\left(h_{0}, \ldots, h_{n}, \kappa\right)$ satisfaz as desigualdades de Poincaré-Hopf se os dados $\left(h_{0}, \ldots, h_{n}, \kappa\right)$ satisfazem as desigualdades de Poincarć-Hopf (2.21) (2.23).

Posteriormente, verificamos que o grafo abstrato de Lyapunov satisfaz as desigualdades de Poincaré-Hopf se e somente se satisfaz as desigualdades generalizadas de Morse para alguma escolha de vetor de números de Betti. Mais ainda, dado um conjunto de números inteiros positivos $\left(h_{0}, \ldots, h_{n}\right)$ que satisfazem as desigualdades de Poincaré-Hopf para variedades fechadas encontramos todos os possíveis vetores de números de Betti que satisfazem as desigualdades de Morse (1) para esse conjunto.

A novidade nas demonstraçōes destes resultados é a utilização das singularidades de índice $k$ do tipo $k$-desconectante e $(k-1)$-conectante que aparecem como as variáveis $h^{c d}$ 's do sistema linear gerado pelo algoritmo de explosāo, associado à continuação. Este sistema linear pode ser visto como um problema de fluxo em rede. Todas as soluções 
deste problema podem ser obtidas por meio de uma solução particular e de circulações elementares nesta rede. A partir destas soluções, estabelccemos um método para construir números de Betti satisfazendo (1) e $\gamma_{1} \geq \kappa$.

Obtemos também propriedades destes conjuntos de desigualdades e igualdades importantes sob o ponto de vista de análise convexa. Estabelecemos que o conjunto de soluçōes nāo-ncgativas de qualquer deles é um poliedro limitado, ou seja um politopo. Sabe-se que um politopo é completamente caracterizado por seus pontos extremos, ou vértices, sendo constituído pelo casco convexo de seus vértices. Freqëntemente, no entanto, o casco convexo dos pontos inteiros pertencentes a um politopo está contido propriamente no politopo. É surpreendente constatar que isto não ocorre com relaçāo aos politopos considerados. Demonstramos que os cascos convexos dos vetores $h^{c d}$ inteiros não negativos e dos vetores inteiros de números de Betti coincidem com os politopos definidos pelos respectivos conjuntos de desigualdades e igualdades. Ao último destes politopos, denominamos politopo de Morse. Outro fato surpreendente é a estreita relação entre estes conjuntos de vetores que, juntamente com a caracterização obtida para os politopos permite obter todos os vetores de números de Betti a partir dos $h^{c d}$ 's e vice-versa.

Este trabalho está dividido nos seguintes capítulos. Capítulo 1 introduzimos algumas definições tal como explosões e implosões de grafos abstratos de Lyapunov que serão necessárias para definir a continuação de um grafo.

No Capítulo 2 introduzimos as desigualdades de Poincaré-Hopf mais gerais (2.16)(2.20) para blocos isolantes e apresentamos o caso particular, as desigualdades de Poincaré-Hopf (2.21)-(2.23) para variedades fechadas. A diferença principal entre estas versões é que as desigualdades de Poincaré-Hopf (2.16)-(2.20) para blocos isolantes $N$ envolvem os números de Betti dos bordos de saída e entrada de $N$.

No Capítulo 3 apresentamos os resultados de continuação de um grafo abstrato de Lyapunov. Tratamos o problema local, isto é, dado um vértice $v$, apresentamos o algoritmo de explosão o qual é se traduz num sistema linear que denotamos por sistema $h_{\kappa}^{\text {cd }}$. Provamos que este sistema tem solução inteira não negativa se e somente se as desigualdades de Poincaré-Hopf (2.16)-(2.20) são satisfeitas no caso $\kappa=0$. Além do mais, produzimos todas as soluções neste caso. Em outras palavras, como cada solução corresponde a uma continuação do semi-grafo de Lyapunov associado ao vértice $v, L_{v}$, apresentamos todas 
as possíveis continuações de $L_{v}$ a $11 \mathrm{~m}$ semi-grafo de Lyapunov do tipo Morse com cycle rank $\kappa=0$.

No Capítulo 4 apresentamos as desigualdades de Morse generalizadas, também conhecidas como desigualdades de Morse-Conley. Estas desigualdades valem para fluxos contínuos $\phi_{t}$ sobre uma $n$-variedade $M$, orientável, fechada, com conjunto recorrente por cadeia $R$ possuindo um número finito de componentes. Cada componente $R_{k}$ é um conjunto invariante isolado e seja $\left(h_{0}\left(R_{k}\right), \ldots, h_{n}\left(R_{k}\right)\right)$ as dimensões dos índices homológicos de Conley de $R_{k}$. Em [5] foi provado que, dado $\phi_{t}$ sobre $M \operatorname{com} R=\cup R_{k}$, $h_{j}=\sum_{R_{k} \subset R} h_{j}\left(R_{k}\right)$ e $\gamma_{i}$ o $i$-ésimo número de Betti de $M$ então temos as seguintes desigualdades de Morse generalizadas:

$$
\begin{aligned}
\gamma_{n}-\gamma_{n-1}+-\ldots \pm \gamma_{2} \pm \gamma_{1} \pm \gamma_{0} & =h_{n}-h_{n-1}+-\ldots \pm h_{2} \pm h_{1} \pm h_{0} \\
\gamma_{n-1}-\gamma_{n-2}+-\ldots \pm \gamma_{2} \pm \gamma_{1} \pm \gamma_{0} & \leq h_{n-1}-h_{n-2}+\ldots \pm h_{2} \pm h_{1} \pm h_{0} \quad(\mathrm{n}) \\
\vdots & \vdots \\
\gamma_{j}-\gamma_{j-1}+\ldots \pm \gamma_{2} \pm \gamma_{1} \pm \gamma_{0} & \leq h_{j}-h_{j-1}+-\ldots \pm h_{2} \pm h_{1} \pm h_{0} \\
\gamma_{j-1}-\gamma_{j-2}+\ldots \pm \gamma_{2} \pm \gamma_{1} \pm \gamma_{0} & \leq h_{j-1}-h_{j-2}+\ldots \pm h_{2} \pm h_{1} \pm h_{0} \\
\vdots & \vdots \\
\gamma_{2}-\gamma_{1}+\gamma_{0} & \leq h_{2}-h_{1}+h_{0} \\
\gamma_{1}-\gamma_{0} & \leq h_{1}-h_{0} \\
\gamma_{0} & \leq h_{0}
\end{aligned}
$$

Nosso trabalho usa fortemente a propriedade da dualidade do índice de Conley. Seja $R_{k}$ uma componente do conjunto recorrente do fluxo $\phi_{t}$ e $\bar{R}_{k}$ uma componente do conjunto recorrente do fluxo reverso $\phi_{-\ell}$. A condição de dualidade do índice de Conley assegura que $h_{j}\left(\bar{R}_{k}\right)=h_{n-j}\left(R_{k}\right)$. Ou melhor, $\bar{h}_{j}=h_{n-j}$. Para maiores detalhes ver [16]. Note que as desigualdades de Morse (1) se mantém para os índices de Conley duais $\bar{h}_{j}$ e nos referimos a estas como desigualdades de Morse duais. Isto corresponde a passar a barra somente sobre o lado direito das desigualdades já que o lado esquerdo se mantém inalterado.

Por outro lado, as desigualdades de Poincaré-Hopf (2.21)-(2.23) apresentam cotas inferiores para os números $h_{j}$ e $h_{n-j}$ com respeito a somas alternadas de diferenças entre $h_{s}$ e seu dual $h_{n-s}$, com $s<j$. Nestas desigualdades não se faz referência ao espaço 
de fase. Neste capítulo provamos um resultado surpreendente, que as desigualdades de Poincaré-Hopf (2.21)-(2.23) com $\kappa=0$ são satisfeitas, se e somente se as desigualdades de Morse são satisfeitas para algum vetor de números de Betti $\left(\gamma_{0}, \ldots, \gamma_{n}\right)$.

Para efeito de ilustração, vejamos um corolário do resultado acima, no caso clássico de fluxos gradientes. Dado um conjunto $\left\{c_{0}, \ldots, c_{n}\right\}$ de números, onde $c_{i}$ representa o número de pontos críticos de índice de Morse $i$, podemos pré-processar estes dados pelas desigualdades de Poincaré-Hopf e afirmar, primeiramente se os dados são possíveis de ser realizados em algum fluxo gradiente em alguma variedade fechada orientável. Segundo, podemos determinar todos os números de Betti que satisfazem as desigualdades de Morse com esses dados pré-fixados. Apresentamos, ainda neste capítulo, o politopo de Morse no caso $\kappa=0$.

No Capítulo 5, tratamos os grafos abstratos de Lyapunov em toda generalidade, isto é, com a presença de ciclos. Mostramos um teorema de continuação para grafos abstratos de Lyapunov com a presença de ciclos o qual generaliza o teorema de continuação do Capítulo 3. Provamos um teorema de equivalência mais geral que o do Capítulo 4. Este resultado afirma que as desigualdades de Poincarć-Hopf (2.21)-(2.23) são satisfeitas se, c somente se, $\gamma_{1} \geq \kappa$ e as desigualdades de Morse são satisfeitas. Finalmente descrevemos o politopo de Morse neste caso. 


\section{Capítulo 1}

\section{Preliminares}

\subsection{Fluxos do tipo gradiente}

Um ponto $x \in M$ é um ponto recorrente por cadeia se dado $\varepsilon>0$ existe uma $\varepsilon$-cadeia de $x$ a $x$, i.e., existem pontos $x=x_{1}, x_{2}, \ldots, x_{n-1}, x_{n}=x$ e $t(i) \geq 1$ tais que

$$
d\left(\phi_{t(i)}\left(x_{i}\right), x_{i+1}\right)<\varepsilon \quad \forall 1 \leq i<n .
$$

Um conjunto de tais pontos é denotado por $\mathcal{R}$ e é chamado um conjunto recorrente por cadeia. Veja Figura 1.1.
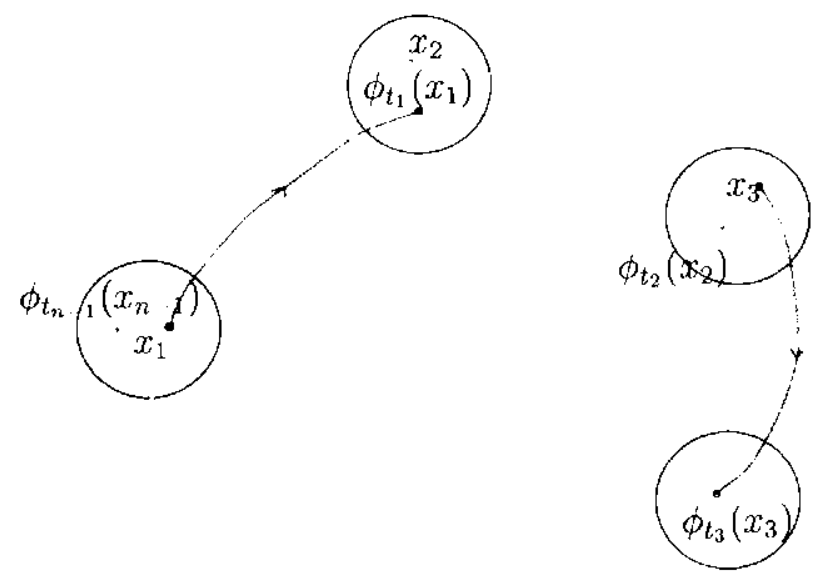

Figura 1.1: $\varepsilon$-cadeia 
Dado um fluxo suave $\phi_{t}: M \rightarrow M$, resultados de Conley implicam que existe uma função suave $f: M \rightarrow \mathbb{R}$ associada a este fluxo com a propricdade que $f$ decresce ao longo das órbitas fora do conjunto recorrente por cadeia $\mathcal{R}$, isto é:

Teorema de Conley Dado um fluxo contínuo $\phi_{t}: M \rightarrow M$, existe uma função contínua $f: M \rightarrow \mathbb{R}$ tal que:

- se $x \notin \mathcal{R}$ então $f\left(\phi_{t}(x)\right)<f\left(\phi_{s}(x)\right)$ sempre que $t>s$;

- se $x, y \in \mathcal{R}$ então $f(x)=f(y)$ se, e somente se, dado $\varepsilon>0$ existem pontos em $\mathcal{R}$, $x=x_{1}, x_{2}, \ldots, x_{n-1}, x_{n}=y, x_{n+1,1}, \ldots, x_{2 n}=x$ e números reais $t(i)>0$ tal que

$$
d\left(\phi_{t(i)}\left(x_{i}\right), x_{i+1}\right)<\varepsilon \quad \forall 1 \leq i<2 n
$$

Esta função é chamada função de Lyapunov. Nos referimos a $\phi_{t}$ como um fluxo do tipo gradiente com respeito a $f$ por causa das propriedades mencionadas no Teorema de Conley que o torna semelhante aos fluxos gradientes. Veja Figura 1.2.

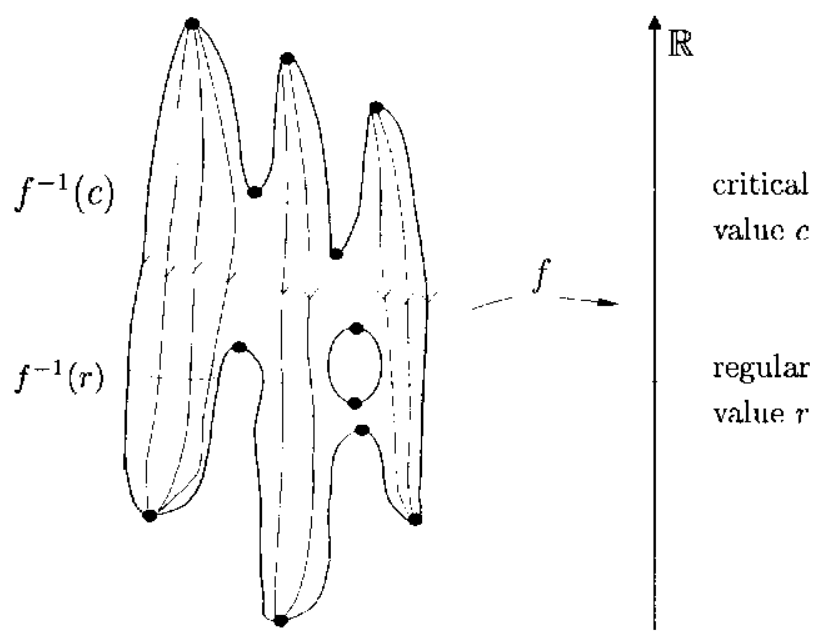

Figura 1.2: Função de Lyapunov. 


\subsection{Grafos de Lyapunov}

Recordemos que uma função de Lyapunov $\int: M \rightarrow \mathbb{R}$ associada a um fluxo, determina um grafo de Lyapunov pela seguinte relação de equivalência sobre $M: x \sim_{f} y$ se, e somente se, $x$ e $y$ pertencem a mesma componente conexa de um conjunto de nível de $f$. Portanto, $M / \sim_{f}$ é um grafo de Lyapunov. É possível escolher $f$ tal que cada nível crítico contém uma única componente $R$ de $\mathcal{R}$. L'm ponto sobre $M / \sim_{f}$ é um ponto de vértice se com respeito a relação de equivalência, correspondc a uma componente conexa do conjunto de nível contendo uma componente $R$ de $\mathcal{R}$. Todos os outros pontos são pontos de aresta. Cada aresta representa uma subvariedade $Q$ de $M$, de codimensão um, cartesiano um intervalo aberto limitado $I, Q \times I$. Para reter algumas informações topológicas de $Q \times I$, as arestas serão rotuladas com os números de Betti de $Q$. Todos os grupos de homologia serão calculados com coeficientes em $\mathbb{Z}_{2}$. Veja Figura 1.3.

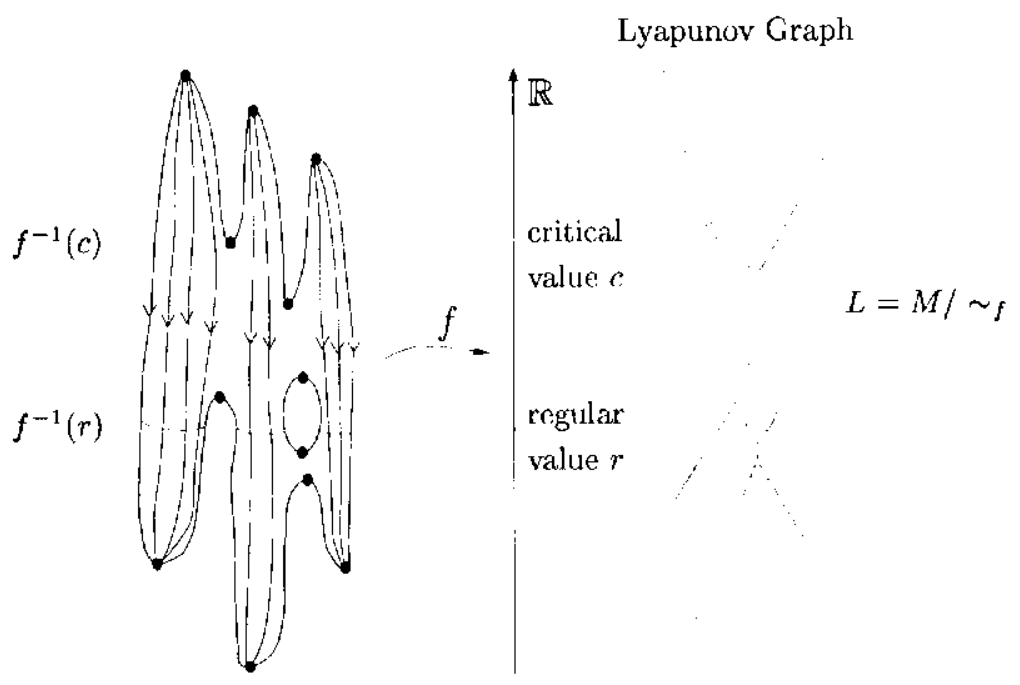

Figura 1.3: Grafo de Lyapunov.

Um grafo abstrato de Lyapunov de dimensăo $n$ é um grafo orientado sem ciclos orientados tal que cada vértice $v$ é rotulado com uma lista de intciros não negativos $\left(h_{0}(v)=k_{0}, \ldots, h_{n}(v)=k_{n}, \kappa(v)\right)$, onde $\kappa$ é o cycle number do vértice. Tambćm, os 
rótulos sobre cada aresta $\left(\beta_{0}=1, \beta_{1}, \ldots, \beta_{n-2}, \beta_{n-1}=1\right)$ deve ser um vetor de números de Betti.

Uma notação alternativa é rotular o vértice com $h_{j}\left(v_{k}\right)=n_{j}$ sempre que $n_{j} \neq 0$ e $k\left(v_{j}\right)=k_{j}$ sempre que $k_{j} \neq 0$. A última notação é mais conveniente sempre que $\left(h_{0}\left(v_{k}\right), \ldots, h_{n}\left(v_{k}\right), \kappa\left(v_{k}\right)\right)$ tenha muitas entradas iguais a zero. Também, por simplicidade omitimos a referência ao vértice $v_{k}$ sempre que possível. Escolhemos rotular as arestas com os números de Betti de uma variedade fechada de dimensão $(n-1)$ que agruparemos num vetor de números de Betti. Um vetor de números de Betti de dimensão $n$ é um vetor de inteiros não negativos com $\left(\gamma_{0}, \gamma_{1}, \ldots, \gamma_{n-1}, \gamma_{n}\right)$ onde $\gamma_{n-k}=\gamma_{k}, \gamma_{0}=\gamma_{n}=1$ e $\gamma_{n / 2}$ é par se $n$ é par. Para simplificar a notação do vetor de números de Betti basta representá-lo até a dimensão média por causa da dualidade de Poincaré. Isto é,

$$
\left(\gamma_{0}, \gamma_{1}, \ldots, \gamma_{n}\right) \leftrightarrow\left(1, \gamma_{1}, \ldots, \gamma_{\left.-\frac{n}{2}\right\rfloor}\right)
$$

\section{Veja Figura 1.4.}

\section{Exemplo}

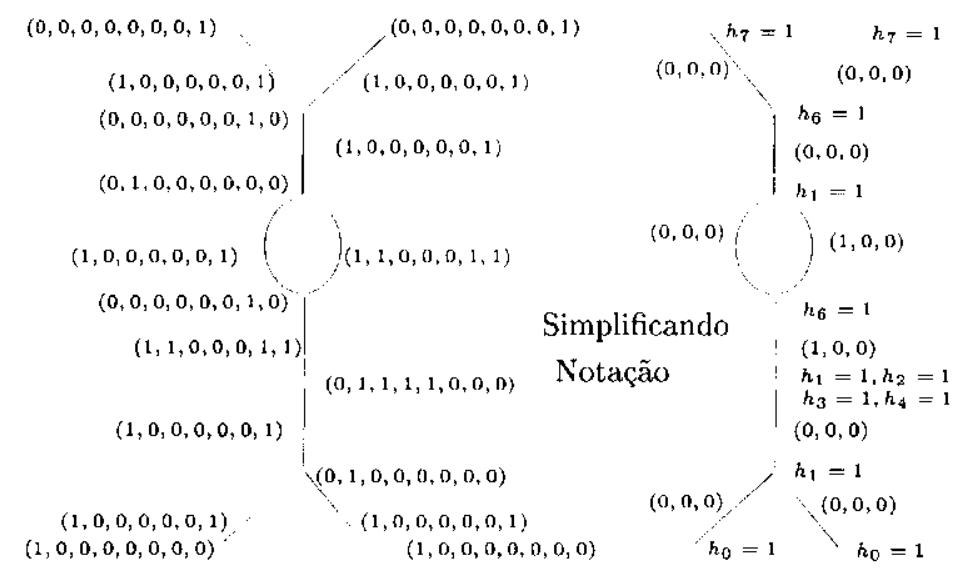

Figura 1.4: Grafo abstrato de Lyapunov em dimensão 7.

Existe uma correspondência natural entre uma $n$-variedade fechada e um grafo abstrato de Lyapunov de dimensão $n$. Para estabelecer a correspondência com o bloco isolante 
precisamos definir um semi-grafo. Para este fim, precisamos estender a noção de um grafo direcionado levando em conta um vértice distinguido, que denotaremos por $\infty$.

Dado um conjunto finito $V$ definimos um semi-grafo direcionado $G^{\prime}=\left(V^{\prime}, E^{\prime}\right)$ como um par de conjuntos $V^{\prime}=V \cup\{\infty\}, E^{\prime} \subset V^{\prime} \times V^{\prime}$. Como sempre, chamamos os elementos de $V^{\prime}$ vértices e os elementos de $E^{\prime}$ considerados como pares ordenados, são chamados arestas direcionadas. Além disso as arestas da forma $(\infty, v)$ e $(v, \infty)$ são chamadas semiarestas (ou arestas dangling como em [9]). Note que sempre que $G^{\prime}$ não contém semiarestas $G^{\prime}$ é um grafo no sentido usual. A representação gráfica do grafo terá as semiarestas representadas como uma aresta com um único vértice.

Um bloco isolante $N$ de um conjunto invariante isolado $\Lambda$ com conjunto de entrada para o fluxo $N^{+}$e conjunto de saída para o fluxo $N^{-}$, pode ser associado a um semi-grafo de Lyapunov $L_{N}$, consistindo de um vértice rotulado com as dimensões da homologia dos índices de Conley de $\Lambda$ e semi-arestas de entrada e saída rotuladas com vetores de números de Betti. O número de arestas incidentes positivamente (negativamente) $e^{+}\left(e^{-}\right)$ corresponde ao número de componentes conexas de $N^{+}\left(N^{-}\right)$. Os rótulos sobre as arestas correspondem aos vetores de números de Betti das subvariedades fechadas de codimensão $\operatorname{um} N^{+}$e $N^{-}$.

\subsection{Teoria de Conley}

Um conjunto $S \subset M$ é invariante se $\phi_{t}(S)=S$ para todo $t \in \mathbb{R}$. Um conjunto compacto $N \subset M$ é uma vizinhança isolante se inv $(\mathrm{N}, \phi)=\left\{\mathrm{x} \in \mathrm{N}: \phi_{\mathrm{t}}(\mathrm{x}) \subset \mathrm{N}, \forall \mathrm{t} \in \mathbb{R}\right\} \subset \operatorname{int} \mathrm{N}$. Um conjunto invariante $S$ é chamado um conjunto invariante isolado se é um conjunto invariante maximal em alguma vizinhança isolante $N$, isto é, $S=\operatorname{inv}(\mathrm{N}, \phi)$. Veja Figura 1.5 .

Um par de espaços compactos $\left(N, N^{-}\right)$é um par-índice se:

- $\operatorname{cl}\left(N-N^{-}\right)$é uma vizinhança isolante para $S$;

- é positivamente invariante em $N$, isto é, se $x \in N^{-}$e $\dot{\phi}_{[0, T]}(x) \subset N$ então $\phi_{[0, T]}(x) \subset$ $N^{-}$ 


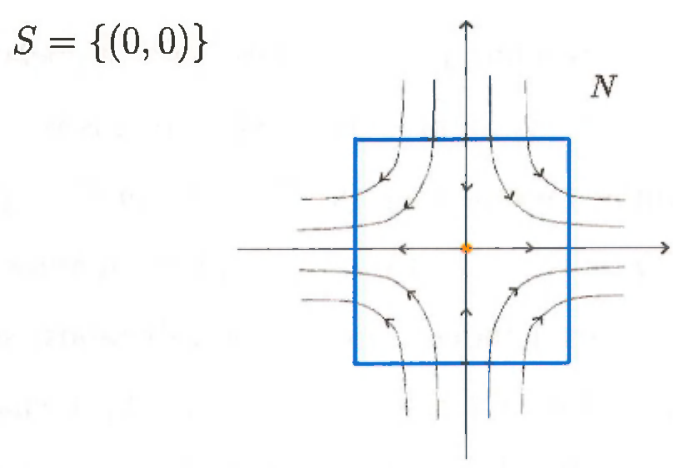

Figura 1.5: Conjunto invariante isolado.

- $N^{-}$é um conjunto de saída para o fluxo, isto é, se $x \in N$ e $\phi_{[0, \infty)}(x) \not \subset N$ então existe um $T>0$ tal que $\phi_{[0, T]}(x) \subset N$ e $\phi_{T}(x) \in N^{-}$.

Veja Figuras 1.6 e 1.7 .
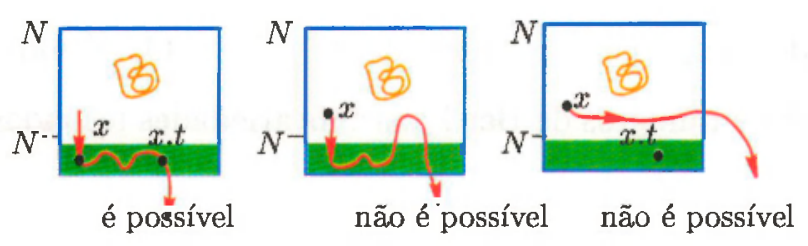

Figura 1.6: Par-índice.

Um conjunto compacto $N$ é um bloco isolante se $N^{-}=\left\{x \in N: \phi_{[0, t)}(x) \not \subset N, \forall t>0\right\}$ é fechado e $\operatorname{inv}(N, \phi) \subset \operatorname{int} N$.

Uma componente $R$ de $\mathcal{R}$ do fluxo $\phi_{t}$ é um exemplo de um conjunto invariante. Trabalharemos sob as hipóteses que $\mathcal{R}$ é a união finita de conjuntos invariantes isolados $R_{i}$. Se $f$ é uma função de Lyapunov associada a um fluxo e $c=f(R)$ então para $\varepsilon>0$, a componente de $f^{-1}[c-\varepsilon, c+\varepsilon]$ que contém $R$ é um bloco isolante para $R$. Veja Figuras 1.8 e 1.9 . 


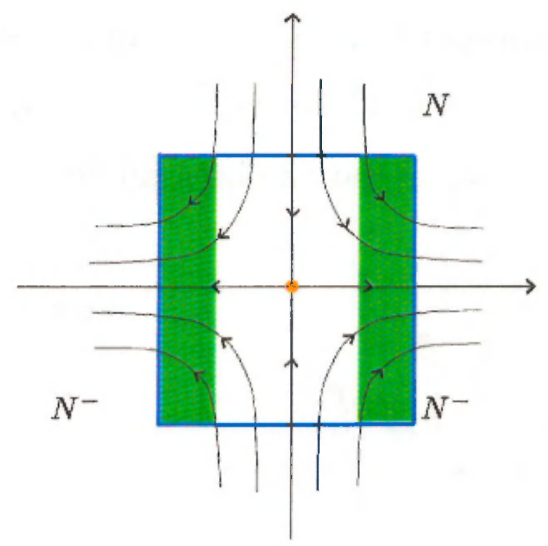

Figura 1.7: Par-Índice
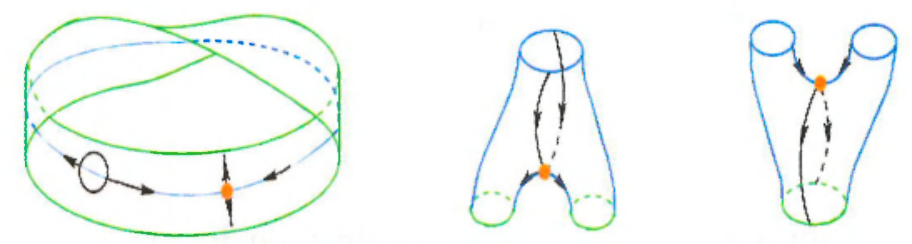

Figura 1.8: Blocos isolantes para selas em dimensão 2.
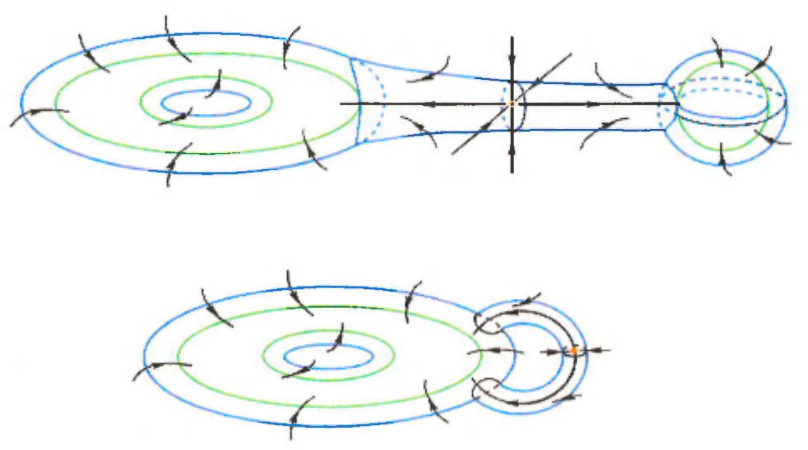

Figura 1.9: Blocos isolantes para selas em dimensão 3.

Tome $\left(N, N^{-}\right)=\left(f^{-1}[c-\varepsilon, c+\varepsilon], f^{-1}(c-\varepsilon)\right)$ como um par índice para $S=R$. O índice de Conley é definido como o tipo de homotopia de $N / N^{-}$e é denotado por $\mathcal{H}(S)$. O índice 
de Conley homológico é denotado por $C H_{*}(S)$ e utilizando coeficientes $\mathbb{Z}_{2}$ denotamos a dimensão de $C H_{*}(S)$ por $h_{*}=\operatorname{dim} \mathrm{CH}_{*}(\mathrm{~S})$. Para maiores detalhes ver [5]. Veja nas Figuras $1.10,1.11,1.12$ e 1.13 os passos para a obtenção de $\mathcal{H}(S)$ onde $S=\{P\}$.

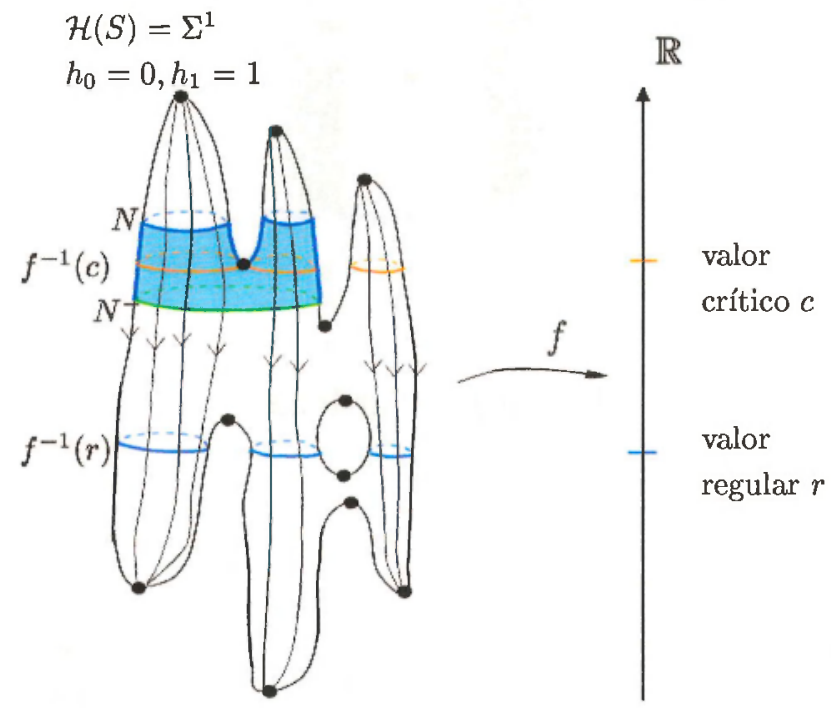

Figura 1.10: Passos para obtenção de $\mathcal{H}(S)$.
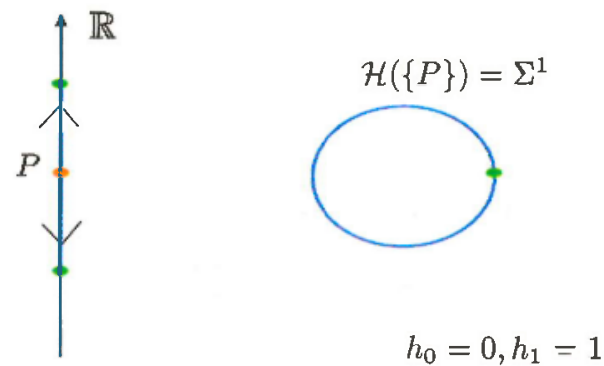

Figura 1.11: Passos para obtenção de $\mathcal{H}(S)$. 


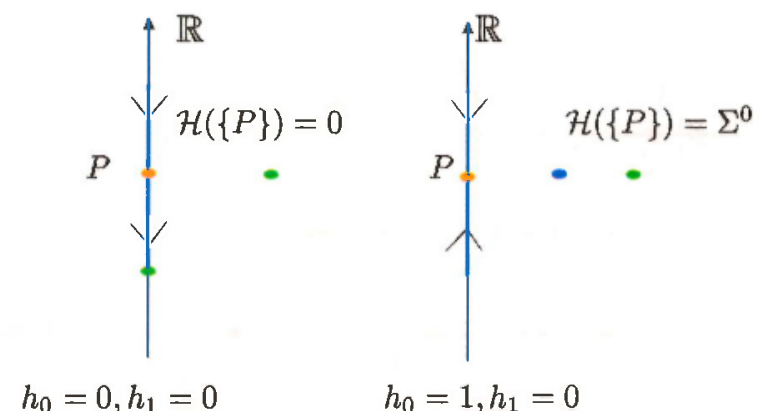

Figura 1.12: Passos para obtenção de $\mathcal{H}(S)$.

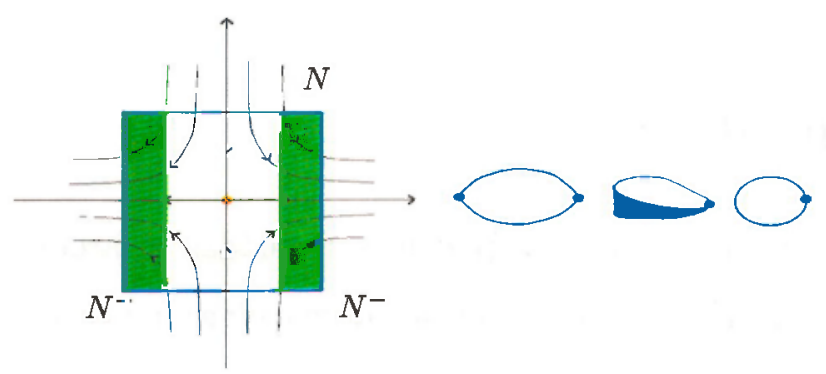

Figura 1.13: Passos para obtenção de $\mathcal{H}(S)$.

$S=\{(0,0)\}$ is invariant set and $\mathcal{H}(S)=\Sigma^{1}$.

Uma propriedade importante do índice de Conley é que se mantém constante para conjuntos relacionados por continuação. Uma família parametrizada de fluxos sobre $M$ é uma coleção de fluxos $\left\{\phi_{t}^{\lambda} \mid \lambda \in I\right\}$ indexados por $I=[0,1]$ tal que $\Phi_{t}(x, \lambda)=\left(\phi_{t}^{\dot{\lambda}} x, \lambda\right)$ é um fluxo suave sobre $M \times I$. Dizemos que $S^{0}$, um conjunto invariante para $\hat{\phi}_{\hat{t}}$, e $S^{1}$, um conjunto invariante para $\phi_{t}^{1}$, estão relacionados por continuação se existe um conjunto invariante isolado $\Sigma \subset M \times I$ para $\Phi_{t}$ tal que $S^{0}=\Sigma \cap\{(x, 0)\}$ e $S^{1}=\Sigma \cap\{(x, 1)\}$. Temos ainda que se $S^{0}$ e $S^{1}$ estão relacionados por continuação então $\mathcal{H}\left(S^{0}\right)=\mathcal{H}\left(S^{1}\right)$.

Temos ainda que se $N$ é uma vizinhança isolante para $\phi_{t}^{\lambda}$ para todo $\lambda \in I$ e $\Sigma=$ $\operatorname{Inv}(N \times I)$ em $\Phi_{t}$, então $\Sigma$ define uma continuação de $\Sigma \cap\{(x, 0)\}$ a $\Sigma \cap\{(x, 1)\}$.

Veja na Figura 1.14 um exemplo de continuação para a seguinte família parametrizada de fluxos definidos na reta: $\dot{x}=x\left(1-x^{2}\right)-\lambda \equiv f(x, \lambda)$ 


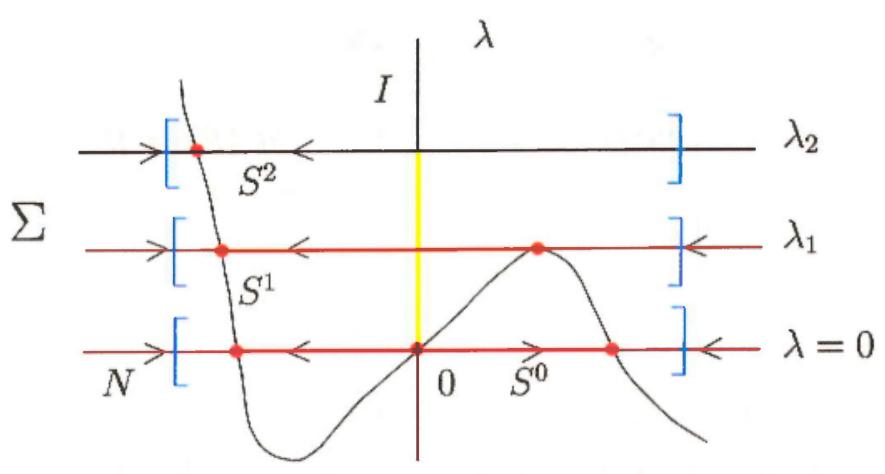

Figura 1.14: Continuação do índice de Conley.

\subsection{Teoria de alças}

Nesta seção descrevemos como as alças podem ser coladas e faremos um resumo das técnicas desenvolvidas em [7]. A principal idéia é desenvolver uma abordagem no espírito da teoria de Morse analisando as mudanças da variedade conforme as alças são coladas. Para este fim, faremos uma conexão entre a colagem de alças e a passagem no grafo de Lyapunov $L$ por um vértice. Os diferentes tipos de colagens das alças corresponderão aos diferentes rótulos dos vértices de $L$.

Denote por $D^{j}$ uma $j$-bola unitária fechada. O bordo de $D^{i}, \partial D^{j}$ é a $(j-1)$-esfera $S^{j-1}$.

Seja $\bar{N}=\bar{N}^{-} \times[0,1]$ a $n$-variedade obtida como um colarinho de $N^{-}$e $H=D^{\ell} \times D^{n-\ell}$. Seja $\theta: \partial D^{\ell} \times D^{n-\ell} \rightarrow \partial \bar{N}$ um mergulho que define a nova variedade $N^{\prime}=N \cup_{\theta} H$, que é o resultado de colar uma $\ell$-alça a $N$. As seguintes notações são usadas $N^{\prime}=N \cup H^{(\ell)}$ ou $N^{\prime}=\bar{N} \cup H$ onde $\partial N^{\prime}=N^{+} \cup N^{-}$. Veja Figura 1.15.

Em termos de um grafo de Lyapunov $L$, a colagem de uma alça corresponde a passagem através de um vértice na direção oposta a orientação do grafo. Logo um vértice de $L$ representa a singularidade. O vértice juntamente com suas arestas incidentes rotuladas representam $N^{\prime}$. As arestas incidentes positivamente representam $N^{+} \times J$ e as arestas incidentes negativamente representam $N^{-} \times J$ onde $J$ é um intervalo limitado aberto. Veja Figura 1.16. 


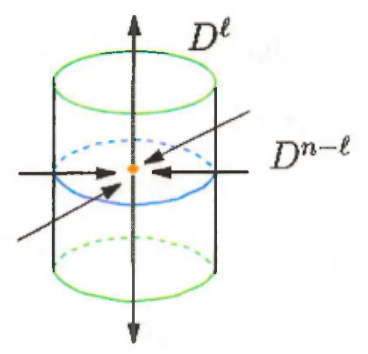

Figura 1.15: Alça $H$ a ser colada.
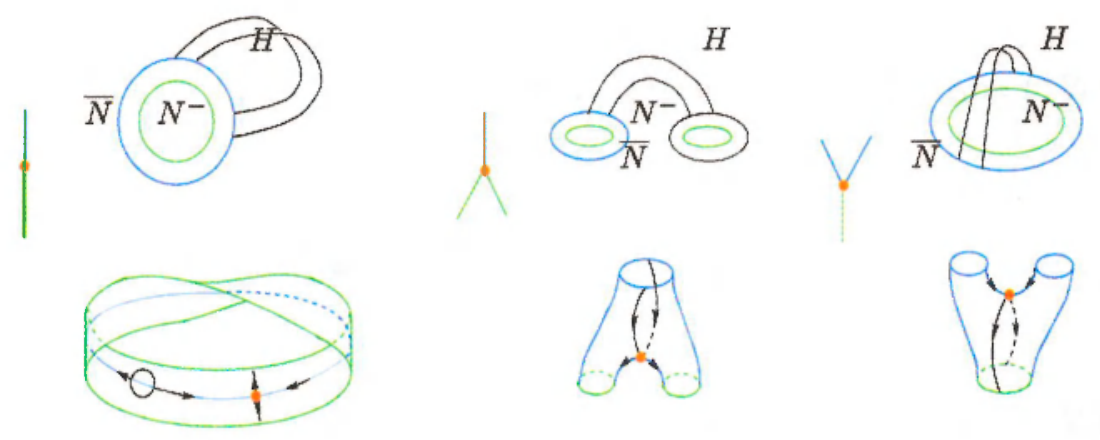

Figura 1.16: Colando alças em dimensão 2

A seguinte definição classifica as alças de acordo com os efeitos algébricos que causam sobre os números de Betti de $N^{+}$uma vez que a alça é colada em um colarinho de $N^{-}$.

Dada uma singularidade não degenerada, uma abordagem clássica é associar à singularidade seu índice de Morse $j$. Mais geralmente, podemos associar as dimensões dos índices homológicos de Conley, $h_{j}=1$ e $h_{k}=0$ para todo $k \neq j$. Em [7] singularidades são classificadas não apenas pelo seu índice, mas também pelo efeito causado sobre os números de Betti dos bordos de entrada e saída, $N^{+}$e $N^{-}$, do fluxo definido sobre um bloco isolante $N$ contendo a singularidade. Em outras palavras, uma alça contendo uma singularidade de índice $\ell$ ou respectivamente, o vértice correspondente sobre $L$ é chamado $\ell$-desconectante, ou simplesmente $\ell$-d, se esta alça tem o efeito algébrico de aumentar o $\ell$-ésimo número de Betti de $N^{+}$ou respectivamente, o correspondente rótulo $\beta_{\ell}$ sobre a 
aresta incidente positivamente. Uma alça contendo uma singularidade de índice $\ell$ ou $o$ correspondente vértice sobre $L$ é chamado $(\ell-1)$-conectante, ou $(\ell-1)$-c, se esta alça tem o efeito algébrico de diminuir o $(\ell-1)$-ésimo número de Betti de $N^{+}$ou respectivamente, o correspondente rótulo $\vec{J}_{\underline{-1}}$ sobre a aresta incidente positivamente. Uma alça contendo uma singularidade de índice $\ell$ ou o vértice correspondente sobre $L$ é chamado $\beta$-invariante, ou $\beta$-i, se todos os números de Betti se mantém constantes. Ver Figura 1.17. Mais adiante utilizamos a notação $h_{j}^{d}$ para representar o número de vértices rotulados com $h_{j}=1$ do tipo $j$-d e $h_{j}^{c}$ o número de vértices rotulados com $h_{j}=1$ do tipo $j-1$-c.

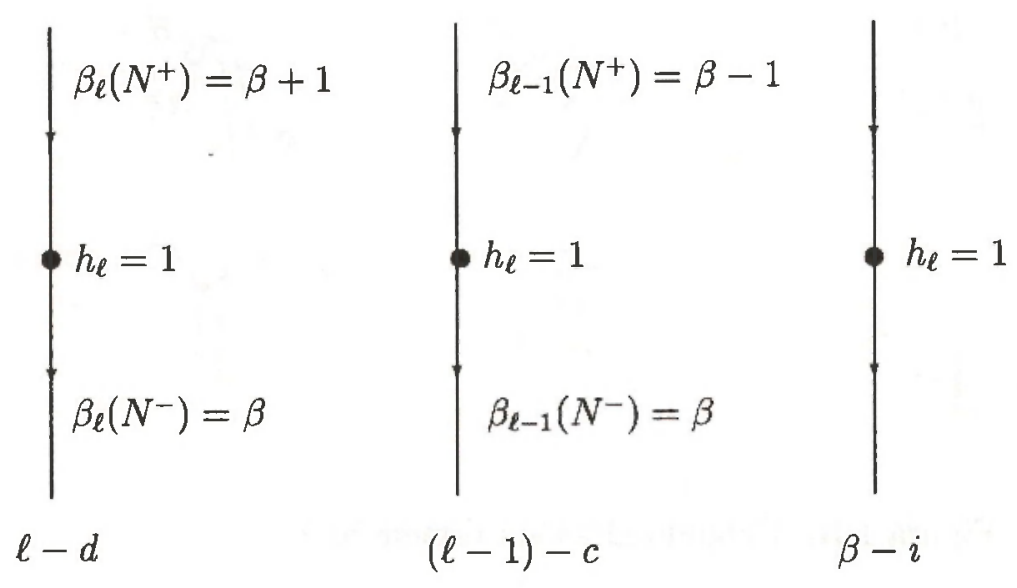

Figura 1.17: Os três possíveis efeitos algébricos.

Os blocos isolantes $N^{\prime}$ de uma singularidade de índice $h_{1}=1 \mathrm{em}$ uma variedade de dimensão 2 do tipo $\beta-i, 0-c$ e $1-d$ são mostrados na Figura 1.18. Ver [11]. Note a presença de uma aresta extra, no caso $0-c$ e $1-d$ precisamente porque os números de Betti que diminuem e aumentam respectivamente controlam o número de componentes conexas. Será mostrado no Teorema 1.4.1 que sobre variedades orientáveis de dimensão $n$ par, singularidades $\beta-i$ podem ocorrer somente se $n=0 \bmod 4$.

Os blocos isolantes $N^{\prime}$ de uma singularidade de índice $h_{1}=1$ correspondendo aos casos 0-c e 1-d e seus respectivos semi-grafos de Lyapunov estão representados na Figura 1.19. Ver [9]. 


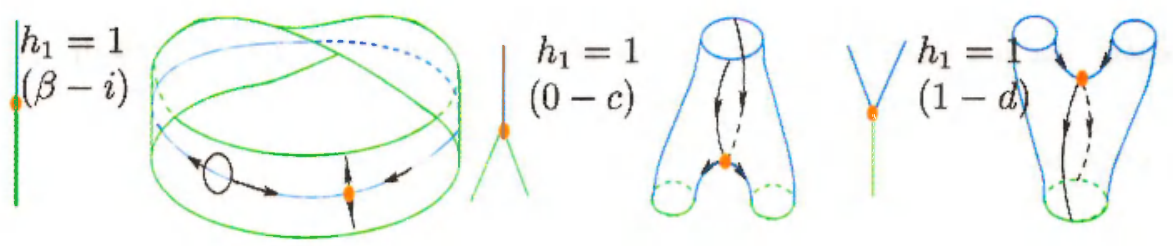

Figura 1.18: Semi-grafos de Lyapunov para selas em dimensão 2

$h_{1}=1$
$(0-c)$

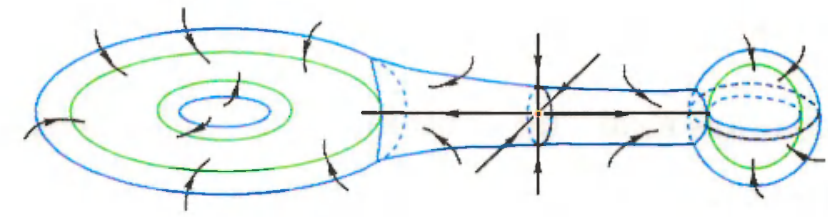

$h_{1}=1$

$(1-d)$

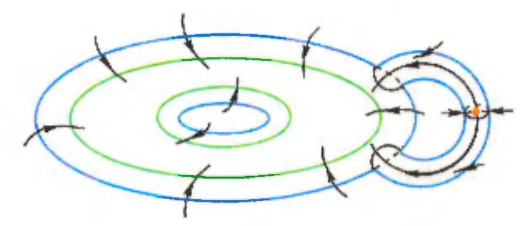

Figura 1.19: Semi-grafos de Lyapunov para selas em dimensão 3.

As arestas incidentes positivamente e negativamente serão rotuladas com o vetor de números de Betti de $N^{-}$e $N^{+}$.

Utilizaremos o seguinte teorema que decorre do resultado principal em [7].

Teorema 1.4.1 Seja $\phi_{t}: M \rightarrow M$ um fluxo de Morse-Smale com função de Lyapunov $f$ : $M \rightarrow \mathbb{R}$. Seja v um vértice do grafo de Lyapunov associado L. Seja $N$ um bloco isolante contendo apenas uma singularidade que corresponde a $v$. Seja $N^{-}, N^{+}$as componentes de entrada e saída de N. Se v é rotulado como uma singularidade de índice $\ell$ então

1. $v$ é $\ell-d,(\ell-1)-c$ ou $\beta-i$;

2. a soma dos rótulos sobre as arestas incidentes positivamente a v (isto é, o número de 
Betti total de $\mathrm{N}^{+}$) muda com respeito a soma dos rótulos sobre as arestas incidentes negativamente a $v$ (isto é, o número de Betti total de $N^{-}$) por \pm 2 ou 0 ;

3. $v$ não pode $\operatorname{ser} \beta$-i se $n \neq 0 \bmod 4$.

Um grafo abstrato de Lyapunov do tipo Morse em dimensão $n$ é um grafo abstrato de Lyapunov que satisfaz as seguintes condições locais:

1. todo vértice é rotulado com $h_{j}=1$ para algum $j=0, \ldots, n$ e o cycle number de cada vértice é igual a zero.

2. o número de arestas incidentes positivamente, $e^{+}$, e o número de arestas incidentes negativamente, $e^{-}$, de um vértice deve satisfazer:

(a) se $h_{j}=1$ para $j \neq 0,1, n-1, n$ então $e^{+}=1$ e $e^{-}=1$;

(b) se $h_{1}=1$ então $e^{+}=1$ e $0<e^{-} \leq 2$; se $h_{n-1}=1$ então $e^{-}=1$ e $0<e^{+} \leq 2$;

(c) se $h_{0}=1$ então $e^{-}=0$ e $e^{+}=1$; se $h_{n}=1$ então $e^{+}=0$ e $e^{-}=1$.

3. todo vértice rotulado com $h_{\ell}=1$ deve ser do tipo $\ell$-d ou $(\ell-1)$-c. Além disso se $n=2 i=0 \bmod 4$ e $h_{i}=1$ então $v$ pode ser rotulado $\operatorname{com} \beta$-i.

Essas condições locais podem melhor ser vistas na Figura 1.20.

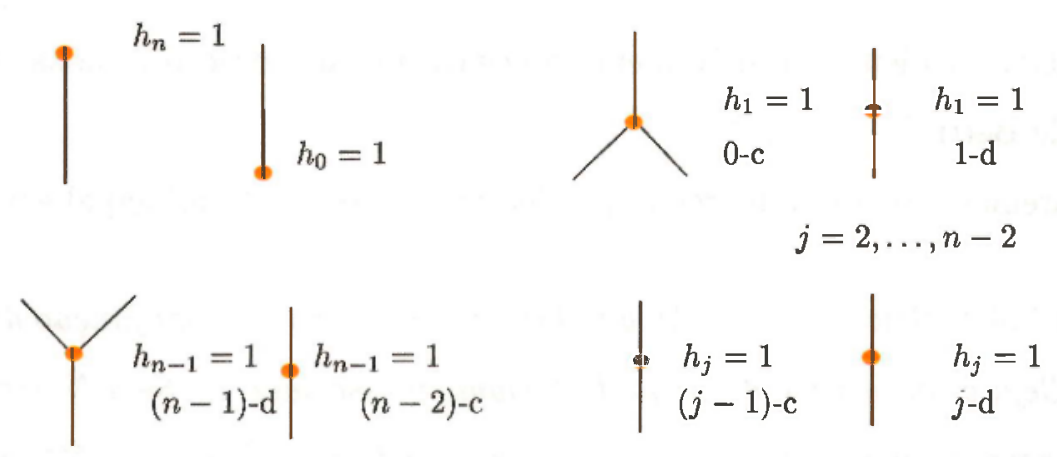

Figura 1.20: Condições locais para um grafo de Lyapunov do tipo Morse. 
Os efeitos algébricos específicos do vértice do tipo $\ell$-d, $(\ell-1)$-c ou $\beta$-i sobre os rótulos $\beta_{\ell}^{+}$das arestas incidentes positivamente ao vértice são especificados em uma série de Proposições em [7]. Seja $v$ um vértice rotulado com $h_{\underline{\ell}}=1 \mathrm{em} L$, onde $L$ representa um fluxo cujo espaço de fase é uma variedade $M$, de dimensão $n$. A dimensão de $M, n$, é referida como a dimensão ambiente. Sempre que $M$ é uma variedade de dimensão par e $\ell$ coincide com sua dimensão média ou sempre que $M$ é uma variedade de dimensão ímpar e $\ell$ coincide com as duas dimensões médias então a análise é um pouco mais elaborada. Estes casos serão referidos como os casos de dimensão média e resumimos os resultados na seguinte proposição.

Proposição 1.4.2 1. Seja $n=2 i$ e $\ell \neq i$ ou $n=2 i+1$ e $\ell \neq i, i+1$. Então

(a) se vé $\ell$-d então

$$
\beta_{k}\left(N^{-}\right)=\left\{\begin{array}{l}
\beta_{k}\left(N^{+}\right) \text {para todo } k \neq \ell, n-\ell-1 \\
\beta_{k}\left(N^{+}\right)-1 \text { para } k=\ell, n-\ell-1
\end{array}\right.
$$

$o u$,

(b) se vé $(\ell-1)-c$ então

$$
\beta_{k}\left(N^{-}\right)=\left\{\begin{array}{l}
\beta_{k}\left(N^{+}\right) \text {para todo } k \neq \ell-1, n-\ell \\
\beta_{k}\left(N^{+}\right)+1 \text { para } k=\ell-1, n-\ell
\end{array}\right.
$$

2. se $n=2 i$ e $\ell=i$ então

(a) se vé $(\beta-\imath)$ então

$$
\beta_{k}\left(N^{-}\right)=\beta_{k}\left(N^{+}\right) \text {para todo } k
$$

(b) se vé $\ell-d$ então

$$
\beta_{k}\left(N^{-}\right)=\left\{\begin{array}{l}
\beta_{k}\left(N^{+}\right) \text {para todo } k \neq i, i-1 \\
\beta_{k}\left(N^{+}\right)-1 \text { para } k=i, i-1
\end{array}\right.
$$

ou,

(c) se vé $((\ell-1)-c)$ então

$$
\beta_{k}\left(N^{-}\right)=\left\{\begin{array}{l}
\beta_{k}\left(N^{+}\right) \text {para todo } k \neq i, i-1 \\
\beta_{k}\left(N^{+}\right)+1 \text { para } k=i, i-1
\end{array}\right.
$$


3. se $n=2 i+1$ e $\ell=i$ então

(a) se vé $(\ell-d)$ então

$$
\beta_{k}\left(N^{-}\right)=\left\{\begin{array}{l}
\beta_{k}\left(N^{+}\right) \text {para todo } k \neq i \\
\beta_{k}\left(N^{+}\right)-2 \text { para } k=i
\end{array}\right.
$$

ou,

(b) se vé $((\ell-1)-c)$ então

$$
\beta_{k}\left(N^{-}\right)=\left\{\begin{array}{l}
\beta_{k}\left(N^{+}\right) \text {para todo } k \neq i+1, i-1 \\
\beta_{k}\left(N^{+}\right)+1 \text { para } k=i+1, i-1
\end{array}\right.
$$

4. se $n=2 i+1$ e $\ell=i+1$ então

(a) se vé $((\ell-1)-c)$ então

$$
\beta_{k}\left(N^{-}\right)=\left\{\begin{array}{l}
\beta_{k}\left(N^{+}\right) \text {para todo } k \neq i \\
\beta_{k}\left(N^{+}\right)+2 \text { para } k=i
\end{array}\right.
$$

ou,

(b) se vé $(\ell-d)$ então

$$
\beta_{k}\left(N^{-}\right)=\left\{\begin{array}{l}
\beta_{k}\left(N^{+}\right) \text {para todo } k \neq i+1, i-1 \\
\beta_{k}\left(N^{+}\right)-1 \text { para } k=i+1, i-1
\end{array}\right.
$$

\subsection{Explosões e implosões de vértices}

Vamos agora estabelecer a noção de explosão de um vértice a fim de definir a continuação de grafos abstratos de Lyapunov. Seja $v$ um vértice sobre um grafo abstrato de Lyapunov rotulado com $\left(h_{0}(v), h_{1}(v) \ldots, h_{n}(v), \kappa_{v}\right)$. Um vértice $v$ pode ser explodido se $v$ pode ser removido e substituído por um grafo abstrato de Lyapunov $I$ do tipo Morse com cycle rank maior ou igual a $\kappa_{v}$. O grafo $I$ deve respeitar as orientações e rótulos das arestas incidentes positivamente e negativamente em $v$. Em outras palavras, o novo grafo obtido deve ser orientado e com cycle rank maior ou igual a $\kappa_{v}$. As arestas incidentes positivamente (negativamente) em $v$, devem ser as arestas incidentes positivamente (negativamente) nos 
vértices de $I$ e os rótulos das arestas devem respeitar as restrições dos vértices do tipo Morse. Além disso,

$$
h_{\lambda}(v)=\sum_{j=1}^{k} h_{\lambda}\left(v_{j}\right), \text { para } \lambda=1, \ldots, n-1, \text { onde } v_{j} \in I
$$

Para ilustrar o uso dos vértices $\ell$-d e $(\ell-1)$-c considere a seguinte explosão de um vértice rotulado com $\left(h_{1}=3, h_{2}=3, h_{3}=2, h_{4}=2, \kappa=0\right)$ e arestas rotuladas com intciros não-negativos como na Figura 1.21. A dimensão ambiente é $n=5$.

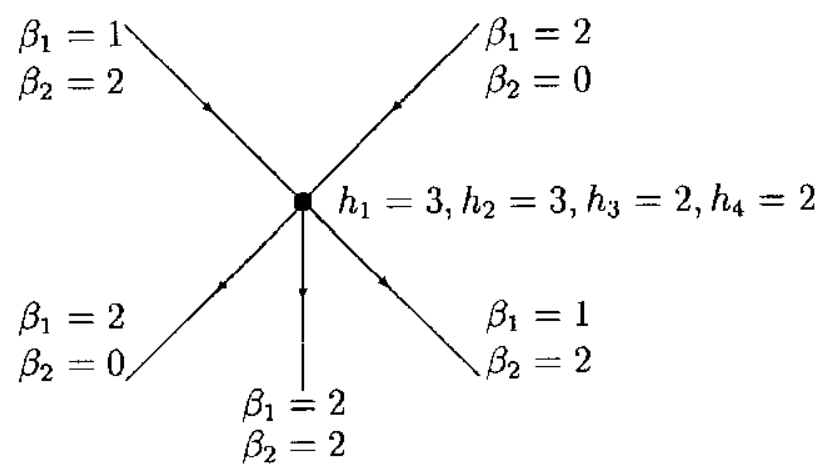

Figura 1.21: Vértice a ser explodido.

Será mostrado que este vértice admite duas diferentes explosões como na Figura 1.22.

Um grafo abstrato de Lyapunov $L\left(h_{0}, h_{1} \ldots, h_{n}, \kappa\right)$ admite uma continuação a um grafo abstrato de Lyapunov do tipo Morse $L_{M}$ se cada vértice pode ser explodido e $L_{M}$ tem cycle rank maior ou igual a $\kappa$.

Dado um grafo abstrato de Lyapunov $L\left(h_{0}, \ldots, h_{n}, \kappa\right)$ com cycle number igual a $\kappa$, podemos definir uma implosão do grafo $L$ como um grafo abstrato de Lyapunov $L_{C}$ com:

1. um vértice do tipo sela $\nu$ rotulado com $\left(h_{1}(\nu), \ldots, h_{n-1}(\nu), \kappa\right)$ onde

$$
\sum_{v \in V} h_{\lambda}(v)=h_{\lambda}(\nu)
$$

onde $V$ é o conjunto de vértices de $L$;

2. o vértice $\nu$ terá $\sum_{v \in V} h_{n}(v)=e^{+}$, arestas incidentes positivamente e terá $\sum_{v \in V} h_{0}(v)=e^{-}$arestas incidentes negativamente; 


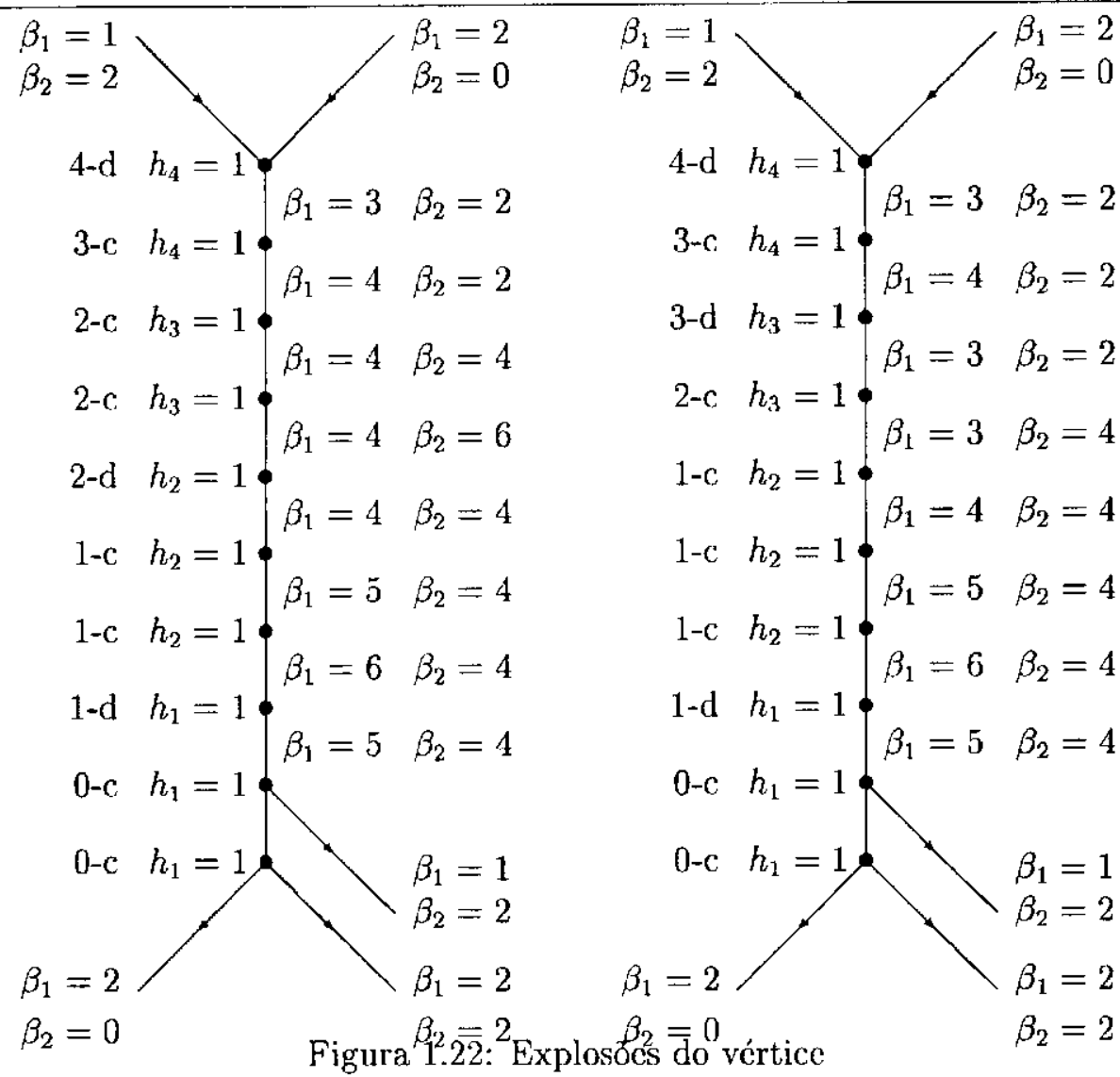

3. as arestas incidentes positivamente de $\nu$ são arestas incidentes negativamente de $e^{+}$ vértices rotulados com $h_{n}=1 \mathrm{e}$ as arestas incidentes negativamente de $\nu$ são arestas incidentes positivamente de $e^{-}$vértices rotulados com $h_{0}=1$;

4. os rótulos de todas as arestas satisfazem $B_{j}^{+}=B_{j}^{-}=0$, para todo $j \neq 0, n-1$, c $B_{j}^{+}=B_{j}^{-}=1$, para $j=0, n-1$. 


\section{Capítulo 2}

\section{Desigualdades de Poincaré-Hopf}

A validade das desigualdades de Poincaré-Hopf, quando testadas nos dados que aparecem nos rótulos de um grafo abstrato de Lyapunov, constitui-se na hipótese fundamental dos resultados principais estabelecidos nesta monografia.

Na Seção (2.1) as Desigualdades de Morse clássicas são relembradas. Na subsequente Seção (2.2) uma filtraçāo de grafo é definida que corresponde a uma filtração induzida pelo fluxo sobre $M$. Isto torna possível transferir informações do fluxo sobre $M$ ao grafo de Lyapunov e vice-versa. Finalmente, esta filtração é usada para provar as desigualdades generalizadas de Morse. Na Seção (2.3) mostramos as desigualdades de Poincaré-Hopf. Estas desigualdades em contraste com as desigualdades de Morse relacionam os índices de homologia de um conjunto invariante isolado aos números de Betti dos conjuntos de entrada e saída de um bloco isolante. As desigualdades de Poincaré-Hopf são deduzidas de uma análise das seqüências exata longa dos pares índice. Devido a filtração do grafo na Subseção (2.2) estes resultados podem ser transferidos ao grafo.

\subsection{Desigualdades de Morse}

A relação entre a topologia de $M$ e os pontos críticos de uma função de valores reais em $M$ são descritas pelas desigualdades de Morse clássicas. Nesta seção resultados básicos são enunciados e maiores detalhes podem ser encontrados em [17]. 
Definição 2.1.1 Seja $S$ uma funçāo de certos pares de espaços nos inteiros. S é subaditiva se dado que $X \supset Y \supset Z$ temos $S(X, Z) \leq S(X, Y)+S(Y, Z)$. $S$ é aditiva se a igualdade se mantém.

Considerando $\beta_{\lambda}(X, Y)$ como o $\lambda$-th número de Betti de $(X, Y)$, isto é, $\beta_{\lambda}(X, Y)=$ $\operatorname{rank}\left(H_{\lambda}(X, Y)\right)$ e $\chi(X, Y)=\sum(-1)^{\lambda} \beta_{\lambda}(X, Y)$ como a característica de Euler, pode ser mostrado analisando a seqüência longa exata de $(X, Y, Z)$ que $\beta_{\lambda}$ é subaditiva e $\chi$ é aditiva.

Lema 2.1.2 Se $S$ é subaditiva e $X_{0} \subset \ldots \subset X_{n}$, então $S\left(X_{n}, X_{0}\right) \leq \sum_{i=1}^{n} S\left(X_{i}, X_{i-1}\right)$. Se $S$ é aditiva então a igualdade se mantém. Se $X_{0}=\emptyset$ então $S\left(X_{n}\right) \leq \sum_{i=1}^{n} S\left(X_{i}, X_{i-1}\right)$ e a igualdade se mantém se $S$ é aditiva.

Demonstração: A prova é feita por indução em $n$.

Dada uma variedade compacta $M$ e uma função diferenciável em $M$ com pontos críticos isolados não-degenerados, defina uma filtração $\emptyset=M^{a_{0}} \subset M^{a_{1}} \subset \ldots \subset M^{a_{k}}=M$, onde $a_{1}<\ldots<a_{k}$. Neste caso, a soma dos ranks de $H_{*}\left(M_{a_{i}}, M_{a_{i-1}}\right)$ é $\sum_{i=1}^{k} \beta_{\lambda}\left(M_{a_{i}}, M_{a_{i-1}}\right)=C_{\lambda}$.

Como $\beta_{\lambda}$ é subaditiva e $\chi$ é aditiva para a filtração $\emptyset=M^{a_{0}} \subset M^{a_{1}} \subset \ldots \subset M^{a_{k}}=M$, os seguintes resultados são obtidos:

1. Desigualdades de Morse fracas

$$
\beta_{\lambda}(M) \leq C_{\lambda}
$$

2. Igualdade de Poincaré-Hopf

$$
\sum(-1)^{\lambda} \beta_{\lambda}(M)=\sum(-1)^{\lambda} C_{\lambda}
$$

onde $C_{\lambda}$ denota o número de pontos críticos de índice $\lambda$.

Defina a função subaditiva $S_{\lambda}(X, Y)=\beta_{\lambda}(X, Y)-\beta_{\lambda-1}(X, Y)+-\ldots \pm \beta_{0}(X, Y)$. As desigualdades de Morse são obtidas usando esta função na filtração $\emptyset=M^{a_{0}} \subset M^{a_{1}} \subset$ $\ldots \subset M^{a_{k}}=M$.

$$
S_{\lambda}(M) \leq \sum_{i=1}^{n} S_{\lambda}\left(M^{a_{i}}, M^{a_{i-1}}\right)=C_{\lambda}-C_{\lambda-1}+-\ldots \pm C_{0}
$$

ou em outras palavras,

$$
\beta_{\lambda}(M)-\beta_{\lambda-1}(M)+-\ldots \pm \beta_{0}(M) \leq C_{\lambda}-C_{\lambda-1}+-\ldots \pm C_{0} .
$$




\subsection{Filtração do grafo}

Nesta seção descrevemos um procedimento de ordenamento parecido a ordem topológica de um grafo orientado, conexo e finito sem ciclos orientados. Dado um fluxo suave sobre $M$ com grafo de Lyapunov $L$ definimos uma filtração sobre $L$. Esta filtração induz uma filtração sobre $M$.

Definição 2.2.1 Seja $v_{1}^{1}, v_{1}^{2}, \ldots, v_{1}^{k_{1}}$ vértices de $L$ de outdegree 0 . Considere as arestas incidentes positivamente a estes vértices. Denote por $N_{1}^{1}, N_{1}^{2}, \ldots, N_{1}^{k_{1}}$ os blocos isolantes associados a estes vértices e arestas. Defina $M_{1}=\bigcup_{i=1}^{k_{1}} N_{1}^{i}$. Conecte todos os vértices $v_{1}^{j}, j=1, \ldots, k_{1}$ com os vértices $v_{2}^{j}, j=1, \ldots, k_{2}$ tal que as arestas incidentes negativamente destes vértices são as arestas incidentes positivamente dos vértices $v_{1}^{j}, j=1, \ldots, k_{1}$. Considere as arestas incidentes positivamente e negativamente dos vértices $v_{2}^{j}, j=1, \ldots, k_{2}$. Denote por $N_{2}^{1}, N_{2}^{2}, \ldots, N_{2}^{k_{2}}$ os blocos isolantes associados a estes vértices e suas arestas. Defina $M_{2}=M_{1} \cup \bigcup_{i=1}^{k_{2}} N_{2}^{i}$. Repita este processo até que todos os vértices do grafo estejam conectados. Isto define uma filtração sobre $M$, $\emptyset=M_{0} \subset M_{1} \subset \ldots \subset M_{\ell}=M$ que será chamada filtração do grafo,

$$
M_{\ell}=M_{\ell-1} \cup \bigcup_{i=1}^{k_{\ell}} N_{\ell}^{i} .
$$

$\mathrm{O}$ índice de Conley homotópico do vértice $v_{i}^{j}$ que representa um conjunto invariante isolado, é denotado por $\mathcal{H}\left(v_{i}^{j}\right)$, e sua homologia $H_{*}\left(\mathcal{H}\left(v_{i}^{j}\right)\right)$ é o índice homológico de Conley do vértice $v_{i}^{j}$ e é denotado por $C H_{*}\left(v_{i}^{j}\right)$. Cada vértice $v_{i}^{j}$ do grafo está rotulado com os ranks dos índices de homologia de Conley, $h_{*}\left(v_{i}^{j}\right)$. Se $C H_{*}\left(v_{i}^{j}\right)$ é trivial então por simplicidade omitimos $h_{*}\left(v_{i}^{j}\right)=0$.

Dado um fluxo suave sobre $M$ com grafo de Lyapunov $L$, uma linha de fluxo sobre $M$ corresponde a um caminho orientado sobre $L$. Se não existe caminho orientado entre dois vértices $v$ e $w$ em $L$ entāo não existe órbita conectante entre $\Lambda_{v}$ e $\Lambda_{w}$.

É importante notar que a escolha da filtração do grafo $\emptyset=M_{0} \subset M_{1} \subset \ldots \subset M_{\ell}=M$ implica que não existe caminho orientado conectando vértices que estejam no mesmo nível $\left\{v_{i}\right\}_{j=1}^{k_{i}}$ e portanto $\Lambda_{i}^{j}$ associado a estes vértices não tem órbitas conectantes. Logo, esta filtração define um par-índice $\left(M_{i}, M_{i-1}\right)$ para os conjuntos invariantes isolados associados 
a $\bigcup_{j=1}^{k_{i}} v_{i}^{j}, \quad \bigcup_{j=1}^{k_{i}} \Lambda_{i}^{j}$. Isto pode facilmente ser visto já que $\left(M_{i}, M_{i-1}\right)$ é um par de espaços compactos tais que:

1. $c l\left(M_{i}, M_{i-1}\right)$ é uma vizinhança isolante para a união disjunta dos conjuntos invariantes isolados $\bigcup_{j=1}^{k_{i}} \Lambda_{i}^{j}$

2. $M_{i-1}$ é positivamente invariante em $M_{i}$, isto é, se $x \in M_{i-1}$ e $\phi_{[0, T]}(x) \subset M_{i}$ entāo $\phi_{[0, T]}(x) \subset M_{i-1}$

3. $M_{i-1}$ é o conjunto de saida para o fluxo, isto é, se $x \in M_{i}$ e $\phi_{[0, \infty]}(x) \not \subset M_{i}$ então existe um $T>0$ tal que $\phi_{[0, T]}(x) \subset M_{i}$ e $\phi_{T}(x) \subset M_{i-1}$ (esta última condiçāo é satisfeita por vacuidade).

Usando a filtração do grafo, a subadilividade de $\beta_{\lambda}$ e a aditividade de $\chi$ como na seção anterior, os seguintes resultados são obtidos:

1. desigualdades de Morse fracas generalizadas

$$
\beta_{\lambda}(M) \leq h_{\lambda}(M)
$$

2. igualdade de Poincaré-Hopf generalizada

$$
\chi(M)=\sum_{\lambda=0}^{n}(-1)^{\lambda} h_{\lambda}(M),
$$

onde $\operatorname{rank}\left(C H_{\lambda}\left(M_{i}, M_{i-1}\right)\right)=h_{\lambda}\left(M_{i}, M_{i-1}\right)$ e $\sum_{i} h_{\lambda}\left(M_{i}, M_{i-1}\right)=h_{\lambda}(M)$.

Defina a funçāo subaditiva $S_{\lambda}$ como na seção anterior e use a filtração do grafo para obter as desigualdades de Morse generalizadas:

$$
S_{\lambda}(M) \leq \sum_{i=1}^{\ell} S_{\lambda}\left(M_{i}, M_{i-1}\right)=h_{\lambda}(M)-h_{\lambda-1}(M)+\ldots \ldots \pm h_{0}(M)
$$

isto é,

$$
\beta_{\lambda}(M)-\beta_{\lambda-1}(M)+-\ldots \pm \beta_{0}(M) \leq h_{\lambda}(M)-h_{\lambda-1}(M)+-\ldots \pm h_{0}(M) .
$$




\subsection{Desigualdades de Poincaré-Hopf}

\subsubsection{Dualidade do índice de Conley}

As desigualdade de Poincaré-Hopf que obtemos usa fortemente a propriedade da dualidade do índice de Conley. Seja $R_{k}$ uma componente do conjunto recorrente do fluxo $\phi_{t}$ e $\bar{R}_{k}$ uma componente do conjunto recorrente do fluxo reverso $\phi_{-t}$. A condição de dualidade do indice de Conley assegura que $h_{j}\left(\bar{R}_{k}\right)=h_{n-j}\left(R_{k}\right)$. Ou melhor, $\bar{h}_{j}=h_{n-j}$. Ver [16].

Existem muitos exemplos de conjuntos invariantes isolados que satisfazem a exigência acima. Por exemplo se um fluxo $\phi_{t}$ tem uma singularidade $P$ de índice de Conley $S^{k}$ $\left(h_{k}=1\right)$, então a singularidade $P$ para o fluxo reverso $\phi_{-t}$ tem índice de Conley $S^{n-k}$ $\left(h_{n-k}=1\right)$. O mesmo se mantém para uma órbita periódica $\gamma$ de um fluxo $\phi_{t}$ com índice de Conley $S^{k} \vee S^{k+1}\left(h_{k}=h_{k+1}=1\right)$. O fluxo reverso $\phi_{-t}$ tem $\gamma$ como uma órbita periódica de índice de Conley $S^{n-k-1} \vee S^{n-k}\left(h_{n-k-1}=h_{n-k}=1\right)$. Logo, o dual de $h_{k}=1$ é $h_{n-k}=1$ e o dual de $h_{k+1}=1$ é $h_{n-(k+1)}=1$.

Isto pode também ser visto em fluxos 3-dimensionais que possuem como conjunto isolado invariante uma suspensāo do subshift do tipo finito $\sigma$ com matriz $A^{n \times n}$. A suspensão será denotada por $\tilde{\sigma}$. O índice homológico de Conley de $\tilde{\sigma}$ é

$$
\begin{gathered}
C H_{1}(\tilde{\sigma})=F^{n} /(I-A) F^{n} \\
C H_{2}(\tilde{\sigma})=\operatorname{ker}(I-A) \text { on } F^{n} .
\end{gathered}
$$

Logo,

$$
h_{1}=\operatorname{dim} C H_{1}(\tilde{\sigma})=\operatorname{dim} F^{n} /(I-A) F^{n}=\operatorname{dim} \operatorname{ker}(I-A)=\operatorname{dim} C H_{2}(\tilde{\sigma})=h_{2} .
$$

Considerando o fluxo reverso, $\tilde{\sigma}^{-1}$ é a suspensão do subshift do tipo finito $\sigma^{-1} \mathrm{com}$ a transposta de $A, A^{t}$ como sua matriz. Também, a suspensão do subshift do tipo finito para o fluxo inverso tem índices homológicos de Conley iguais a:

$$
\begin{gathered}
C H_{1}\left(\tilde{\sigma}^{-1}\right)=F^{n} /\left(I-A^{t}\right) F^{n} \\
C H_{2}\left(\tilde{\sigma}^{-1}\right)=\operatorname{ker}\left(I-A^{t}\right) \text { on } F^{n}
\end{gathered}
$$

Logo, dado que $\bar{h}_{i}$ representa a dimensão do índice homológico de Conley para o fluxo reverso,

$$
\bar{h}_{1}=\operatorname{dim} F^{n} /\left(I-A^{t}\right) F^{n}=\operatorname{dim} \operatorname{ker}\left(I-A^{t}\right)=\check{h}_{2} .
$$


Como $\operatorname{ker}\left(I-A^{t}\right) \cong \operatorname{ker}(I-A)$, isto implica que $h_{1}=h_{2}=\bar{h}_{1}=\vec{h}_{2}$. Portanto, $h_{1}$ é dual a $\vec{h}_{2}$.

\subsubsection{Seqüência longa exata de um par índice}

Seja $\left(N, N^{-}\right)$um par-índice para $\Lambda$ e $\left(N, N^{+}\right)$um par-índice para $\Lambda^{\prime}$.

Considere a seguinte seqüência longa exata para os pares $\left(N, N^{-}\right)$e $\left(N, N^{+}\right)$:

$$
\begin{aligned}
& 0 \rightarrow H_{n}\left(N^{-}\right) \stackrel{i_{n}}{\longrightarrow} H_{n}(N) \stackrel{p_{n}}{\longrightarrow} H_{n}\left(N, N^{-}\right) \stackrel{\partial_{n}}{\longrightarrow} H_{n-1}\left(N^{-}\right) \stackrel{i_{n-1}}{\longrightarrow} H_{n-1}(N) \stackrel{p_{n-1}}{\longrightarrow} \\
& \rightarrow H_{n-1}\left(N, N^{-}\right) \stackrel{\partial_{n-1}}{\longrightarrow} H_{n-2}\left(N^{-}\right) \stackrel{i_{n-2}}{\longrightarrow} H_{n-2}(N) \stackrel{p_{n-2}}{\longrightarrow} H_{n-2}\left(N, N^{-}\right) \stackrel{\partial_{n-2}}{\longrightarrow} \ldots \\
& \stackrel{\partial_{4}}{\rightarrow} H_{3}\left(N^{-}\right) \stackrel{i_{3}}{\rightarrow} H_{3}(N) \stackrel{p_{3}}{\rightarrow} H_{3}\left(N, N^{-}\right) \stackrel{\partial_{3}}{\rightarrow} H_{2}\left(N^{-}\right) \stackrel{i_{2}}{\rightarrow} H_{2}(N) \stackrel{p_{2}}{\longrightarrow} H_{2}\left(N, N^{-}\right) \stackrel{\partial_{2}}{\longrightarrow} \\
& \rightarrow H_{1}\left(N^{-}\right) \stackrel{i_{1}}{\rightarrow} H_{1}(N) \stackrel{p_{1}}{\rightarrow} H_{1}\left(N, N^{-}\right) \stackrel{\partial_{1}}{\rightarrow} H_{0}\left(N^{-}\right) \stackrel{i_{0}}{\rightarrow} H_{0}(N) \stackrel{p_{0}}{\rightarrow} H_{0}(N, N) \rightarrow 0 \\
& 0 \rightarrow H_{n}\left(N^{+}\right) \stackrel{i_{n}^{\prime}}{\longrightarrow} H_{n}(N) \stackrel{p_{n}^{\prime}}{\longrightarrow} H_{n}\left(N, N^{+}\right) \stackrel{\partial_{n}^{\prime}}{\longrightarrow} H_{n-1}\left(N^{+}\right) \stackrel{i_{n-1}^{\prime}}{\longrightarrow} H_{n-1}(N) \stackrel{p_{n-1}^{\prime}}{\longrightarrow} \\
& \rightarrow H_{n ! 1}\left(N, N^{+}\right) \stackrel{\partial_{n-1}^{\prime}}{\longrightarrow} H_{n-2}\left(N^{+}\right) \stackrel{i_{n-2}^{\prime}}{\longrightarrow} H_{n-2}(N) \stackrel{p_{n-2}^{\prime}}{\longrightarrow} H_{n-2}\left(N, N^{+}\right) \stackrel{\partial_{n-2}^{\prime}}{\longrightarrow} \ldots \\
& \stackrel{\partial_{4}^{\prime}}{\longrightarrow} H_{3}\left(N^{+}\right) \stackrel{i_{3}^{\prime}}{\longrightarrow} H_{3}(N) \stackrel{p_{3}^{\prime}}{\longrightarrow} H_{3}\left(N, N^{+}\right) \stackrel{\partial_{3}^{\prime}}{\longrightarrow} H_{2}\left(N^{+}\right) \stackrel{i_{2}^{\prime}}{\longrightarrow} H_{2}(N) \stackrel{p_{2}^{\prime}}{\longrightarrow} H_{2}\left(N, N^{+}\right) \stackrel{\partial_{2}^{\prime}}{\longrightarrow} \\
& H_{1}\left(N^{+}\right) \stackrel{i_{1}^{\prime}}{\rightarrow} H_{1}(N) \stackrel{p_{1}^{\prime}}{\rightarrow} H_{1}\left(N, N^{+}\right) \stackrel{\partial_{1}^{\prime}}{\rightarrow} H_{0}\left(N^{+}\right) \stackrel{i_{0}^{\prime}}{\rightarrow} H_{0}(N) \stackrel{p_{0}^{\prime}}{\rightarrow} H_{0}\left(N, N^{+}\right) \rightarrow 0
\end{aligned}
$$

É um resultado elementar provar que dado uma seqüência longa exata de espaços vetoriais,

$$
\stackrel{h}{\rightarrow} A \stackrel{i}{\rightarrow} B \stackrel{j}{\rightarrow} C \stackrel{k}{\rightarrow} \ldots \ldots \rightarrow D \rightarrow 0
$$

rank $\operatorname{Im} h+\operatorname{rank} \operatorname{Im} i=\operatorname{rank} A$. Isto segue do fato que $\operatorname{rank} A=\operatorname{rank} \operatorname{Im} i+\operatorname{rank} \operatorname{ker} i$ e da exatidão da seqüência $\operatorname{ker} i=\operatorname{Im} h$. Logo,

$$
\operatorname{rank} h=\operatorname{rank} A-\operatorname{rank} B+\operatorname{rank} C-+\ldots \pm \operatorname{rank} D \geq 0
$$

Assumimos também que $h_{i}=0$ for $i=0, n$. Esta última hipótese será removida no final da seção. Aplicando estes argumentos à sequiência longa exata dos pares $\left(N, N^{-}\right)$e $\left(N, N^{+}\right)$, e considerando $H_{n}(N)=H_{n}\left(N^{ \pm}\right)=0, \operatorname{rank} H_{i}\left(N, N^{-}\right)=h_{i}, \operatorname{rank} H_{i}\left(N, N^{+}\right)=$ 
$h_{n-i}, \operatorname{rank} H_{0}\left(N^{-}\right)=e^{-}, \operatorname{rank} H_{0}\left(N^{+}\right)=e^{+}, \operatorname{rank} H_{0}(N)=1 \mathrm{e} \operatorname{rank}\left(H_{i}\left(N^{ \pm}\right)\right)=B_{i}^{ \pm}$, obtemos a seguinte equaçāo:

$$
\begin{gathered}
\operatorname{rank} \partial_{n}=B_{n-1}^{-}-\operatorname{rank}\left(H_{n-1}(N)\right)+h_{n-1}-B_{n-2}+\operatorname{rank}\left(H_{n-2}(N)\right)-h_{n-2} \ldots \\
\left.\left. \pm B_{3}^{-} \pm \operatorname{rank}\left(H_{3} N\right)\right) \pm h_{3} \pm B_{2}^{-} \pm \operatorname{rank}\left(H_{2} N\right)\right) \pm h_{2} \\
\pm B_{1}^{-} \pm \operatorname{rank}\left(H_{1}(N)\right) \pm h_{1} \pm e^{-} \pm 1
\end{gathered}
$$

Na subseção seguinte, a análise da seqüência longa exata dos pares será considerada em dois casos separados, quando dim $N$ é ímpar ou par. No primeiro caso, a igualdade de Poincaré-Hopf clássica é obtida.

\subsubsection{Caso de dimensão ímpar, $n=2 i+1$.}

A dualidade de Poincaré sobre o bordo de $N$ implica que $B_{2 i-j}^{-}=B_{j}^{-}$. Também, como rank $\partial_{2 i+1}=0$, segue de (2.3) que:

$$
\begin{gathered}
2 e^{-}-2 B_{1}^{-}+2 B_{2}^{-}-+\ldots \pm 2 B_{i-1} \pm B_{i}^{-}+h_{2 i}-h_{2 i-1}+\ldots-h_{3}+h_{2}-h_{1}= \\
=\sum_{j=0}^{2 i}(-1)^{j} \operatorname{rank}\left(H_{j}(N)\right)
\end{gathered}
$$

Analogamente, usando a sequiência longa exata do par $\left(N, N^{+}\right)$e usando a dualidade dos índices, $h_{j}(\Lambda)=h_{(2 i+1)-j}\left(\Lambda^{\prime}\right)$ a seguinte equação se mantém:

$$
\begin{gathered}
2 e^{+}-2 B_{1}^{+}+2 B_{2}^{+}-+\ldots \pm 2 B_{i-1}^{+} \pm B_{i}^{+}+h_{1}-h_{2}+-\ldots-h_{2 i-2}+h_{2 i-1}-h_{2 i}= \\
=\sum_{j=0}^{2 i}(-1)^{j} \operatorname{rank}\left(H_{j}(N)\right)
\end{gathered}
$$

Subtraindo (2.4) de (2.5) e dividindo por dois, temos a seguinte equação:

$$
\begin{gathered}
\left(e^{+}-e^{-}\right)-\left(B_{1}^{+}-B_{1}^{-}\right)+\ldots \pm\left(B_{i-1}^{+}-B_{i-1}^{-}\right) \pm \frac{\left(B_{i}^{+}-B_{i}^{-}\right)}{2} \\
-h_{2 i}+h_{2 i-1}-+\ldots \pm h_{i} \pm \ldots-h_{2}+h_{1}=0
\end{gathered}
$$


que pode ser representada por:

$$
\mathcal{B}^{+}-\mathcal{B}^{-}=e^{-}-e^{+}+\sum_{j=1}^{2 i}(-1)^{j} h_{j}
$$

onde,

$$
\begin{aligned}
& \mathcal{B}^{+}=\frac{(-1)^{i}}{2} B_{i}^{+} \pm B_{i-1}^{+} \pm \ldots-B_{1}^{+} \\
& \mathcal{B}^{-}=\frac{(-1)^{i}}{2} B_{i}^{-} \pm B_{i-1}^{-} \pm \ldots-B_{1}^{-}
\end{aligned}
$$

Isto é uma forma alternativa da igualdade de Poincaré-Hopf clássica. See [7] para maiores detalhes.

A fim de atingir nossos objetivos, é suficiente analisar a seqüência longa exata dos pares começando na dimensão média.

Analisando os ranks de $p_{i}$ em (2.1), temos o seguinte:

$$
\begin{gathered}
\operatorname{rank} p_{i}=h_{i}-B_{i-1}^{-}+\operatorname{rank}\left(H_{i-1}\right)-h_{i-1}+B_{i-2}^{-}-\operatorname{rank}\left(H_{i-2}\right)+h_{i-2}-+\ldots \\
\pm B_{2}^{-} \pm \operatorname{rank}\left(H_{2}\right) \pm h_{2} \pm B_{1}^{-} \pm \operatorname{rank}\left(H_{1}\right) \pm h_{1} \pm e^{-} \pm 1 \geq 0 \\
\Rightarrow h_{i} \geq B_{i-1}^{-}-B_{i-2}^{-}+-\ldots \pm B_{2}^{-} \pm B_{1}^{-}+h_{i-1}-h_{i-2}+-\ldots \pm h_{2} \pm h_{1} \pm e^{-} \pm 1 \\
-\operatorname{rank}\left(H_{i-1}\right)+\operatorname{rank}\left(H_{i \cdots 2}\right)-+\ldots \pm \operatorname{rank}\left(H_{2}\right) \pm \operatorname{rank}\left(H_{1}\right)
\end{gathered}
$$

Analogamente, considerando rank $\partial_{i}^{\prime}$ em (2.2) e usando a dualidade dos índices, temos a seguinte desigualdade:

$-\operatorname{rank}\left(H_{i-1}\right)+\operatorname{rank}\left(H_{i-2}\right)-+\ldots \pm \operatorname{rank}\left(H_{2}\right) \pm \operatorname{rank}\left(H_{1}\right) \geq-B_{i-1}^{+}+B_{i-2}^{+}-+\ldots \pm B_{2}^{+} \pm B_{1}^{+}$

$$
-h_{i+2}-+\ldots \pm h_{2 i-1} \pm h_{2 i} \pm e^{+} \pm 1
$$

Substituindo (2.9) em (2.8) temos a seguinte desigualdade:

$$
h_{i} \geq-\left(B_{i-1}^{+}-B_{i-1}^{-}\right)+\left(B_{i-2}^{+}-B_{i-2}^{-}\right)+-\ldots \pm\left(B_{2}^{+}-B_{2}^{-}\right) \pm\left(B_{1}^{+}-B_{1}^{-}\right)
$$




$$
-\left(h_{i+2}-h_{i-1}\right)+\left(h_{i+3}-h_{i-2}\right)+-\ldots \pm\left(h_{2 i-1}-h_{2}\right) \pm\left(h_{2 i}-h_{1}\right) \pm\left(e^{+}-e^{-}\right)
$$

Analogamente analisando $p_{i}^{\prime}$ e $\partial_{i}$ como acima e usando a dualidade dos índices, a seguinte desigualdade é obtida:

$$
\begin{gathered}
h_{i+1} \geq-\left[-\left(B_{i-1}^{+}-B_{i-1}^{-}\right)+\left(B_{i-2}^{+}-B_{i-2}^{-}\right)+-\ldots \pm\left(B_{2}^{+}-B_{2}^{-}\right) \pm\left(B_{1}^{+}-B_{1}^{-}\right)\right. \\
\left.-\left(h_{i+2}-h_{i-1}\right)+\left(h_{i+3}-h_{i-2}\right)+\ldots \ldots \pm\left(h_{2 i-1}-h_{2}\right) \pm\left(h_{2 i}-h_{1}\right) \pm\left(e^{+}-e^{-}\right)\right]
\end{gathered}
$$

Note que na equação (2.10) $h_{i}$ é maior ou igual a um número inteiro e na equação (2.11), $h_{i+1}$ é maior ou igual a este mesmo número com sinal oposto. Obviamente, como $h_{i}$ e $h_{i+1}$ são inteiros nāo negativos, uma das desigualdades é redundante. Isto continuará ocorrer nas desigualdades seguintes.

O restante das desigualdades serão obtidas analisando dois pares de aplicações ao mesmo tempo,

$$
\left\{\left[\left(p_{i-1}, \partial_{i-1}^{\prime}\right),\left(p_{i-1}^{\prime}, \partial_{i-1}\right)\right], \ldots\left[\left(p_{2}, \partial_{2}^{\prime}\right),\left(p_{2}^{\prime}, \partial_{2}\right)\right]\right\}
$$

Note que a análise destes pares produz as desigualdades tanto no caso de dimensão ímpar, $n=2 i+1$, como no caso de dimensão par, $n=2 i$, e serão escritas com respeito a $n$.

Logo, o último par a ser analisado é $\left(p_{2}, \partial_{2}^{\prime}\right)$ e $\left(p_{2}^{\prime}, \partial_{2}\right)$. A análise do primeiro par implica que:

$$
h_{2} \geq-\left(B_{1}^{+}-B_{1}^{-}\right)-\left(h_{n-1}-h_{1}\right)+\left(e^{+}-e^{-}\right)
$$

enquanto a análise do último:

$$
h_{n-2} \geq-\left[-\left(B_{1}^{+}-B_{1}^{-}\right)-\left(h_{n-1}-h_{1}\right)+\left(e^{+}-e^{-}\right)\right]
$$

As últimas desigualdades são obtidas analisando $p_{1}$ e $p_{1}^{\prime}$ :

$$
\begin{gathered}
e^{-} \leq h_{1}+1 \\
e^{+} \leq h_{n-1}+1
\end{gathered}
$$


A análise das seqüências longa exatas dos blocos isolantes produz uma coleção de desigualdades, igualdade (2.7) e desigualdades $(2.10),(2.11)$ e sucessivamente até as desigualdades (2.12), (2.13) e (2.14) que serão chamadas desigualdades de Poincaré-Hopf para o caso de dimensão ímpar.

\subsubsection{Caso de dimensão par, $n=2 i$.}

Nesta seção, as desigualdades são obtidas da mesma maneira, isto é, analisando as sequiências longa exatas dos pares $\left(N, N^{ \pm}\right)$onde $\operatorname{dim} N=2 i$.

As desigualdades serão obtidas analisando dois pares de aplicações ao mesmo tempo,

$$
\left\{\left[\left(p_{i}, \partial_{i}^{\prime}\right),\left(p_{i}^{\prime}, \partial_{i}\right)\right], \ldots\left[\left(p_{2}, \partial_{2}^{\prime}\right),\left(p_{2}^{\prime}, \partial_{2}\right)\right]\right\} .
$$

As últimas desigualdades são obtidas analisando $p_{1}$ e $p_{1}^{\prime}$.

Portanto, as desigualdades de Poincaré-Hopf para o caso de dimensão par são idênticas ao caso de dimensão ímpar exceto na dimensão média:

$$
\left\{\begin{aligned}
h_{i} \geq & -\left(B_{i-1}^{+}-B_{i-1}^{-}\right)+\left(B_{i-2}^{+}-B_{i-2}^{-}\right)+-\ldots \pm\left(B_{2}^{+}-B_{2}^{-}\right) \pm\left(B_{1}^{+}-B_{1}^{-}\right) \\
& -\left(h_{i+1}-h_{i-1}\right)+\left(h_{i+2}-h_{i-2}\right)+-\ldots \pm\left(h_{2 i-2}-h_{2}\right) \pm\left(h_{2 i-1}-h_{1}\right) \pm\left(e^{+}-e^{-}\right) \\
& \\
h_{i} \geq & -\left[-\left(B_{i-1}^{+}-B_{i-1}^{-}\right)+\left(B_{i-2}^{+}-B_{i-2}^{-}\right)+-\ldots \pm\left(B_{2}^{+}-B_{2}^{-}\right) \pm\left(B_{1}^{+}-B_{1}^{-}\right)\right. \\
& \left.-\left(h_{i+1}-h_{i-1}\right)+\left(h_{i+2}-h_{i-2}\right)+-\ldots \pm\left(h_{2 i-2}-h_{2}\right) \pm\left(h_{2 i-1}-h_{1}\right) \pm\left(e^{+}-e^{-}\right)\right]
\end{aligned}\right.
$$

Além disso, no caso $n=2 \bmod 4$ é necessário exigir que

$$
h_{i}-\sum_{j=1}^{i-1}(-1)^{j+1}\left(B_{j}^{+}-B_{j}^{-}\right)-\sum_{j=1}^{i-1}(-1)^{j}\left(h_{2 i-j}-h_{j}\right)+\left(e^{-}-e^{+}\right) \text {seja par. }
$$

É interessante notar que a igualdade de Poincaré-Hopf não existe no caso de dimensāo par.

Removendo a restrição $h_{n}=h_{0}=0$ obtemos as desigualdades de Poincaré-Hopf em toda sua generalidade. Essencialmente adicionamos ou subtraímos o termo $\left(h_{n}-h_{0}\right)$ nas desigualdades (2.10)-(2.15) e nas igualdades (2.7) e (2.16). Logo, as desigualdades de Poincaré-Hopf gerais são representadas por (2.17), (2.18) e (2.20) abaixo.

$$
\mathcal{B}^{+}-\mathcal{B}^{-}=e^{-}-e^{+}+\sum_{j=0}^{2 i+1}(-1)^{j} h_{j}
$$


onde,

$$
\begin{aligned}
& \mathcal{B}^{+}=\frac{(-1)^{i}}{2} B_{i}^{\dagger} \pm B_{i-1}^{+} \pm \ldots-B_{i}^{+} \\
& \mathcal{B}^{-}=\frac{(-1)^{i}}{2} B_{i}^{-} \pm B_{i-1}^{-} \pm \ldots-B_{1}^{-}
\end{aligned}
$$

$h_{i}-\sum_{j=1}^{i-1}(-1)^{j+1}\left(B_{j}^{+}-B_{j}^{-}\right)-\sum_{j=0}^{i-1}(-1)^{j}\left(h_{2 i-j}-h_{j}\right)+\left(e^{-}-e^{+}\right)$ser par, para $2 i=2 \bmod 4$.

A desigualdade de Poincaré-Hopf no caso $n=2$ é

$$
\left\{h_{1}-h_{2}-h_{0}+2-\left(e^{+}+e^{-}\right) \geq 0 \text { e par. }{ }^{1}\right.
$$

Esta desigualdade (2.19) aparece em [11].

Agora, vamos considerar estas desigualdades com a presença de um parâmetro $\kappa$ associado ao bloco isolante $N$. Assim, as desigualdades de Poincaré-Hopf para blocos isolantes

${ }^{1}$ Se $M$ é não orientável a desigualdade de Poincarć-Hopf é a mesma, contudo a expressão do lado direito da desigualdade (2.19) nāo precisa ser par. 
com este parâmetro será a coleção de desigualdades abaixo:

$$
\begin{aligned}
& \left\{n=2 i+1\left\{\begin{aligned}
& h_{i} \geq-\left(B_{i-1}^{+}-B_{i-1}^{-}\right)+\left(B_{i-2}^{+}-B_{i-2}^{-}\right)+\ldots \ldots \pm\left(B_{2}^{+}-B_{2}^{-}\right) \pm\left(B_{1}^{+}-B_{1}^{-}\right) \\
&-\left(h_{i+2}-h_{i-1}\right)+\left(h_{i+3}-h_{i-2}\right)+-\ldots \pm\left(h_{2 i-1}-h_{2}\right) \\
& \pm\left(h_{2 i}-h_{1}\right) \pm\left[\left(h_{2 i+1}-h_{0}\right)+\left(e^{+}-e^{-}\right)\right] \\
& \\
& h_{i+1} \geq-\left[-\left(B_{i-1}^{+}-B_{i-1}^{-}\right)+\left(B_{i-2}^{+}-B_{i-2}^{-}\right)+\ldots \pm\left(B_{2}^{+}-B_{2}^{-}\right) \pm\left(B_{1}^{+}-B_{1}^{-}\right)\right. \\
& \\
&-\left(h_{i+2}-h_{i-1}\right)+\left(h_{i+3}-h_{i-2}\right)+-\ldots \pm\left(h_{2 i-1}-h_{2}\right) \\
&\left. \pm\left(h_{2 i}-h_{1}\right) \pm\left[\left(h_{2 i+1}-h_{0}\right)+\left(e^{+}-e^{-}\right)\right]\right]
\end{aligned}\right.\right. \\
& \left(h_{i} \geq-\left(B_{i-1}^{+}-B_{i-1}^{-}\right)+\left(B_{i-2}^{+}-B_{i-2}^{-}\right)+\ldots \ldots \pm\left(B_{2}^{+}-B_{2}^{-}\right) \pm\left(B_{1}^{+}-B_{1}^{-}\right)\right. \\
& -\left(h_{i+1}-h_{i-1}\right)+\left(h_{i+2}-h_{i-2}\right)+-\ldots \\
& \pm\left(h_{2 i-2}-h_{2}\right) \pm\left(h_{2 i-1}-h_{1}\right) \pm\left[\left(h_{2 i}-h_{0}\right)+\left(e^{+}-e^{-}\right)\right] \\
& n=2 i \\
& h_{i} \geq-\left[-\left(B_{i-1}^{+}-B_{i-1}^{-}\right)+\left(B_{i-2}^{+}-B_{i-2}^{-}\right)+-\ldots \pm\left(B_{2}^{+}-B_{2}^{-}\right) \pm\left(B_{1}^{+}-B_{1}^{-}\right)\right. \\
& -\left(h_{i+1}-h_{i-1}\right)+\left(h_{i+2}-h_{i-2}\right)+-\ldots \\
& \left. \pm\left(h_{2 i-2}-h_{2}\right) \pm\left(h_{2 i-1}-h_{1}\right) \pm\left[\left(h_{2 i}-h_{0}\right)+\left(e^{+}-e^{-}\right)\right]\right] \\
& \left(h_{j} \geq-\left(B_{j-1}^{+}-B_{j-1}^{-}\right)+\left(B_{j-2}^{+}-B_{j-2}^{-}\right)+-\ldots \pm\left(B_{2}^{+}-B_{2}^{-}\right) \pm\left(B_{1}^{+}-B_{1}^{-}\right)\right. \\
& -\left(h_{n-(j-1)}-h_{j-1}\right)+\left(h_{n-(j-2)}-h_{j-2}\right)+-\ldots \\
& \pm\left(h_{n-1}-h_{1}\right) \pm\left[\left(h_{n}-h_{0}\right)+\left(e^{+}-e^{-}\right)\right] \\
& h_{n-j} \geq-\left[-\left(B_{j-1}^{+}-B_{j-1}^{-}\right)+\left(B_{j-2}^{+}-B_{j-2}^{-}\right)+-\ldots \pm\left(B_{2}^{+}-B_{2}^{-}\right) \pm\left(B_{1}^{+}-B_{1}^{-}\right)\right. \\
& -\left(h_{n-(j-1)}-h_{j-1}\right)+\left(h_{n-(j-2)}-h_{j-2}\right)+-\ldots \\
& \left. \pm\left(h_{n-1}-h_{1}\right)+\left[\left(h_{n}-h_{0}\right)+\left(e^{+}-e^{-\cdots}\right)\right]\right] \\
& \left\{\begin{array}{l}
h_{2} \geq-\left(B_{1}^{+}-B_{1}^{-}\right)-\left(h_{n-1}-h_{1}\right)+\left(h_{n}-h_{0}\right)+\left(e^{+}-e^{-}\right) \\
h_{n-2} \geq-\left[-\left(B_{1}^{+}-B_{1}^{-}\right)-\left(h_{n-1}-h_{1}\right)+\left(h_{n}-h_{0}\right)+\left(e^{+}-e^{-}\right)\right]
\end{array}\right. \\
& \int h_{1}>h_{n}-1+e^{-}+\kappa
\end{aligned}
$$




\subsection{Desigualdades de Poincaré-Hopf para variedades fechadas}

$\mathrm{Na}$ seção anterior as desigualdades de Poincaré-Hopf em toda a sua generalidade são introduzidas para fluxos em blocos isolantes $N$. Estas desigualdades envolvem os números de Betti dos bordos de entrada e saída de $N$.

Estas mesmas desigualdades podem ser obtidas num caso particular das desigualdades de Poincaré-Hopf para blocos isolantes que denominaremos por desigualdades de PoincaréHopf para variedades fechadas (2.21), (2.22) e (2.23). Estas desigualdades apresentam cotas inferiores sobre os números $h_{j}$ com respeito as somas alternadas de diferenças entre $h_{s}$ e $h_{n-s}$ para $s<j$.

$$
\left\{\begin{array}{l}
\left\{\begin{array}{l}
n=2 i+1\left\{-h_{i} \leq\left(h_{i+2}-h_{i-1}\right)-\left(h_{i+3}-h_{i-2}\right)+-\ldots \pm\left(h_{2 i}-h_{1}\right) \pm\left(h_{2 i+1}-h_{0}\right) \leq h_{i+1}\right. \\
n=2 i\left\{-h_{i} \leq\left(h_{i+1}-h_{i-1}\right)-\left(h_{i+2}-h_{i-2}\right)+-\ldots \pm\left(h_{2 i-2}-h_{2}\right) \pm\left(h_{2 i}-h_{0}\right) \leq h_{i}\right.
\end{array}\right. \\
\left\{\begin{array}{c}
-h_{j} \leq\left(h_{n-(j-1)}-h_{j-1}\right)-\left(h_{n-(j-2)}-h_{j-2}\right)+\cdots \pm\left(h_{n-1}-h_{1}\right) \pm\left(h_{n}-h_{0}\right) \leq h_{n-j}(\mathrm{j}) \\
\vdots \\
-h_{2} \leq\left(h_{n-1}-h_{1}\right)-\left(h_{n}-h_{0}\right) \leq h_{n-2}(2) \\
\left\{\begin{array}{l}
h_{1} \geq h_{0}-1+\kappa \\
h_{n-1} \geq h_{n}-1+\kappa
\end{array}\right.
\end{array}(1)\right.
\end{array}\right.
$$

No caso $n=2 i+1$ temos

$$
\sum_{j=0}^{2 i+1}(-1)^{j} h_{j}=0
$$

e no caso $n=2 i=2 \bmod 4$ temos

$$
h_{i}-\sum_{j=0}^{i-1}(-1)^{j}\left(h_{2 i-j}-h_{j}\right) \text { be even. }
$$

Estas desigualdades sāo obtidas da seguinte forma. Dado um fluxo contínuo qualquer numa variedade, considere um grafo de Lyapunov abstrato $L\left(h_{0}, \ldots, h_{n}, \kappa\right)$ associado ao 
fluxo. Seja $L_{C}$ a implosão do grafo $L$ e $\nu$ o único vértice do tipo sela de $L_{C}$. O vértice do tipo sela $\nu$ é rotulado com $\left(h_{1}(\nu), \ldots, h_{n \cdots 1}(\nu), \kappa\right)$ onde

$$
\sum_{v \in V} h_{\lambda}(v)=h_{\lambda}(\nu)
$$

onde $V$ é o conjunto de vértices. Observe que $h_{0}(\nu)=0$ e $h_{n}(\nu)=0$. O vértice $\nu$ tem $e^{+}=$ $\sum_{v \in V} h_{n}(v)=h_{n}$, arestas incidentes positivamente e tem $e^{-}=\sum_{v \in V} h_{0}(v)=h_{0}$ arestas incidentes negativamente. Os rótulos de todas as arestas incidentes ao vértice $\nu$ satisfazem $B_{j}^{+}=B_{j}^{-}=0$, para todo $j \neq 0, n-1$, e $B_{j}^{+}=B_{j}^{-}=1$, para $j=0, n-1$. Substituindo estes dados nas desigualdades de Poincaré-Hopf para blocos isolantes (2.17), (2.18) e (2.20), obtemos as desigualdades de Poincaré-Hopf para variedades fechadas (2.21), (2.22) $\mathrm{e}(2.23)$. 


\section{Capítulo 3}

\section{Continuação de Grafos de Lyapunov}

O objetivo deste capítulo é descrever sob quais condições um grafo abstrato de Lyapunov pode ser continuado a um grafo abstrato de Lyapunov do tipo Morse. Estas condições sāo as desigualdades de Poincaré-Hopf para blocos isolantes as quais mostramos serem necessárias e suficientes para a continuaçāo ocorrer.

No Capítulo 1 definimos a explosão de um vértice a fim de definirmos a continuação de grafos abstratos de Lyapunov. Lembremos que um grafo abstrato de Lyapunov admite uma continuação a um grafo abstrato de Lyapunov do tipo Morse se cada vértice pode ser explodido.

A explosão de um vértice é apresentada por meio de um algoritmo construtivo. Apresentamos o número de possíveis explosões para o vértice.

Embora o algoritmo considere a inserção de $\kappa$ ciclos no processo de explosão, neste capítulo não estaremos interessados no controle dos mesmos, ou seja, estamos considerando $\kappa=0$. Dessa forma, explosões com ciclos podem ocorrer, mas esse controle só será feito no Capítulo 5 onde tratamos o grafo em toda sua generalidade. Assim, para facilitar omitimos $\kappa$ nos resultados.

Anunciamos o teorema principal deste capítulo.

Teorema 3.0.1 Todo grafo abstrato de Lyapunov que satisfaz as desigualdades de Poincaré-Hopf em cada vértice pode ser continuado a um grafo abstrato de Lyapunov do tipo Morse.

Este capítulo está dividido em três seçōes. Na Seção (3.1) um algoritmo para a explosāo 
de um vértice é apresentado. $\mathrm{O}$ algoritmo descreve o número dos diferentes tipos de vértices que substituirão $v$. Este algoritmo será um pouco diferente se o vértice está num grafo abstrato de Lyapunov de dimensāo ímpar, de dimensão par igual a $0 \bmod 4$ ou de dimensāo par igual a $2 \bmod 4$. Cada passo do algoritmo impōe uma série de restrições sobre o números e o tipo de vértices que substituirāo $v$ na explosão que são descritas por equações lineares. Assim formamos três famílias de sistemas lineares, cada qual produz pelo menos uma solução que representa o número de diferentes tipos de vértices que substituirão $v$ na explosão.

$\mathrm{Na}$ Seção (3.2) os sistemas lineares serão tratados rigorosamente usando técnicas de programaçāo linear inteira. Em outras palavras será provado que as três diferentes famílias de sistemas lineares sempre tem soluçāo inteira não negativa se certas desigualdades são satisfeitas. Mais ainda, o número de soluções é obtido.

$\mathrm{Na}$ Seção (3.3) é mostrado que as desigualdades na Seção (3.2) requerida para a existência de soluções inteira nāo negativa são precisamente as desigualdades de Poincaré-Hopf.

\subsection{Algoritmo da Explosão}

Nesta seção será apresentado um algoritmo para a explosão de um vértice. Primeiramente faremos uma explosão parcial, que consiste em explodir um vértice a um vértice do tipo sela. Os três passos seguintes do algoritmo não dependem da paridade de $n$. Contudo, o quarto passo distinguirá três casos: $n$ ímpar, $n=0 \bmod 4, n=2 \bmod 4$, produzindo três algoritmos diferentes. Cada algoritmo gera um sistema linear $h_{\kappa}^{c d}$ cujas soluções são precisamente o número de diferentes vértices $(\ell-\mathrm{d}, \ell$-c, $\beta$-i) do tipo Morse usado na explosão do vértice $v$. A última subseção trata o caso especial $n=2$.

\subsubsection{Explosão Parcial de um vértice}

Nesta seção consideramos a explosão parcial de um vértice do tipo sela generalizada, vértices repulsor e atrator. Esta explosão parcial consiste em explodir um tal vértice a um vértice do tipo sela. 
Um vértice num grafo abstrato de Lyapunov rotulado com $\left(h_{0}, h_{1}, \ldots, h_{n}, \kappa\right)$ é um vértice repulsor se tem gran de entrada zero e $h_{n}>0$ (respectivamente, é um vértice atrator se tem grau de saída zero e $h_{0}>0$ ). Caso contrário, um vértice com grau de entrada e grau de saída positivo é um vértice do tipo sela generalizada. Um caso particular deste último caso ocorre quando o vértice é rotulado com $\left(h_{0}=0, h_{1}, \ldots, h_{n}=0, \kappa\right) \mathrm{e}$ o denotaremos por vértice do tipo sela. O algoritmo da explosão parcial consiste em executar uma explosão sobre um vértice que o reduz a um vértice do tipo sela.

\section{Caso 1 - Vértice do tipo sela generalizada}

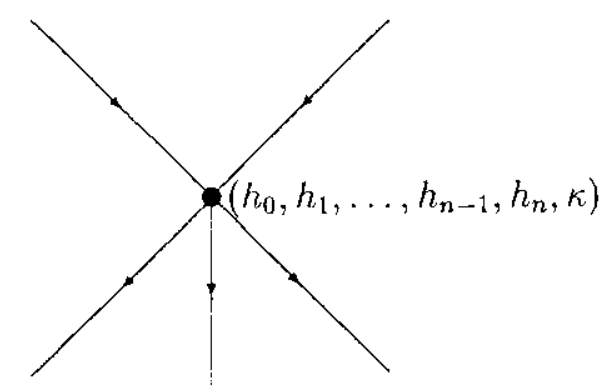

Figura 3.1: Vértice do tipo sela generalizada.

Dado um vértice do tipo sela generalizada rotulado $\operatorname{com}\left(h_{0}, h_{1}, \ldots, h_{n}, \kappa\right)$, uma explosão parcial será feita para obter um vértice do tipo sela rotulado com $\left(0, h_{1}, \ldots, h_{n-1}, 0, \kappa\right)$.

A explosão parcial consiste em acrescentar ao vértice os semi-grafos $G_{0}^{+}$e $G_{0}^{-}$como segue:

1. $G_{0}^{+}$é formado com $h_{n}$ vértices rotulados com $h_{n}=1$ e $h_{n}-1$ vértices rotulados com $h_{n-1}=1$ do tipo $(n-1)$-d e com uma aresta incidente negativamente que conecta a $v$.

2. $G_{0}^{-}$é formado com $h_{0}$ vértices rotulados $\operatorname{com} h_{0}=1 \mathrm{e} h_{0}-1$ vértices rotulados com $h_{1}=1$ do tipo 0 -c e com uma aresta incidente positivamente em $v$. 
Agora, o vértice $v$ tem $e^{+}+1$ arestas incidentes positivamente e $e^{-}+1$ arestas incidentes negativamente. Mais ainda, o vértice está agora rotulado com

$$
\left(h_{n}=0, h_{n-1}-\left(h_{n}-1\right), h_{n \cdot 2}, \ldots, h_{3}, h_{2}, h_{1}-\left(h_{0}-1\right), h_{0}=0, \kappa\right) .
$$

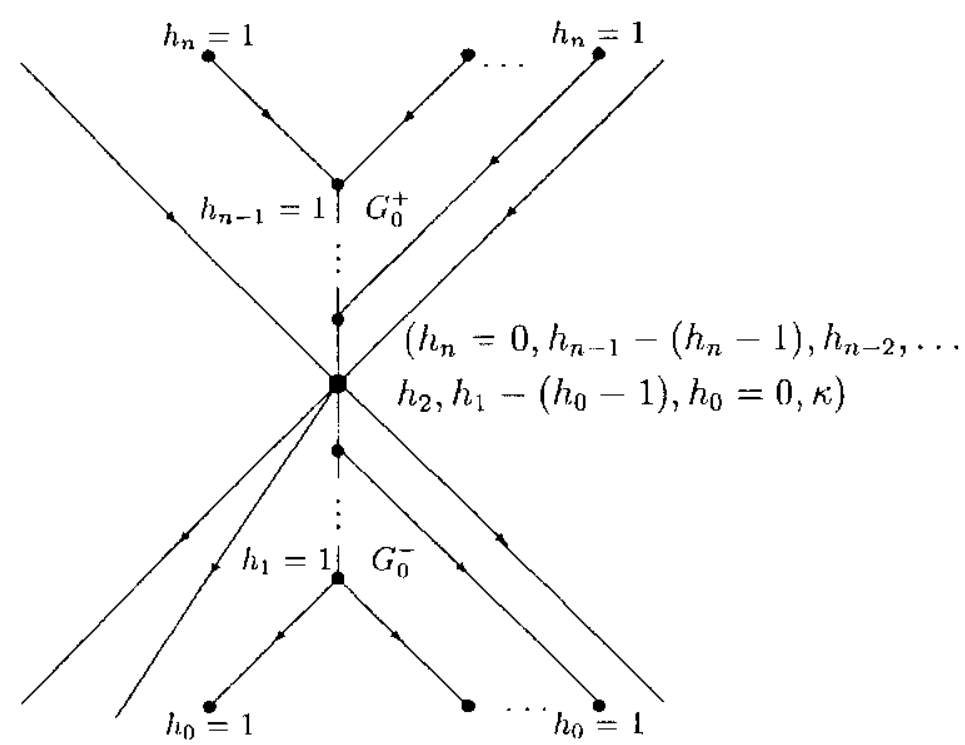

Figura 3.2: Explosão partial de um vértice do tipo sela generalizada.

Observe que este vértice parcialmente explodido satisfaz as desigualdades de PoincaréHopf para blocos isolantes. É fácil ver que isto se segue substituindo os novos rótulos para o vértice parcialmente explodido, isto é, $h_{n-1}$ por $h_{n-1}-\left(h_{n}-1\right), h_{1}$ por $h_{1}-\left(h_{0}-1\right)$, $e^{+}$por $e^{+}+1$ e $e^{-}$por $e^{-}+1$, em (2.7), (2.10), (2.11) no caso de dimensão ímpar ou (2.15), (2.16) no caso de dimensão par e sucessivamente até as desigualdades (2.12), (2.13) e (2.14), obtemos as desigualdades gerais de Poincaré-Hopf (2.17), (2.18) e (2.20).

\section{Caso 2 - Vértices atrator and repulsor}

Considere um vértice repulsor, $e^{\dagger}=0$, rotulado $\mathrm{com}\left(h_{0}, h_{1}, \ldots, h_{n}, \kappa\right)$, uma explosão parcial será feita para obter um vértice rotulado com $\left(0, h_{1}, \ldots, h_{n-1}, 0, \kappa\right)$.

Os grafos $G_{0}^{+}$e $G_{0}^{-}$serão definidos como no caso anterior.

Agora, o vértice $v$ tem 1 aresta incidente positivamente e $e^{-}+1$ arestas incidentes 


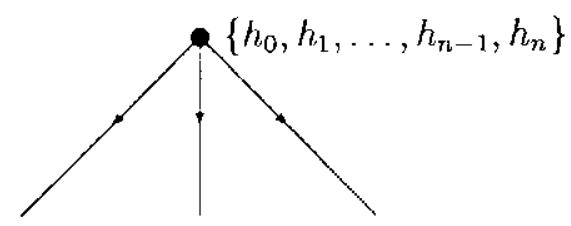

Figura 3.3: Vértice Repulsor.

negativamente. Além disso, o vértice está agora rotulado com

$$
\left(h_{n}=0, h_{n-1}-\left(h_{n}-1\right), h_{n-2}, \ldots, h_{3}, h_{2}, h_{1}-\left(h_{0}-1\right), h_{0}=0, \kappa\right) .
$$

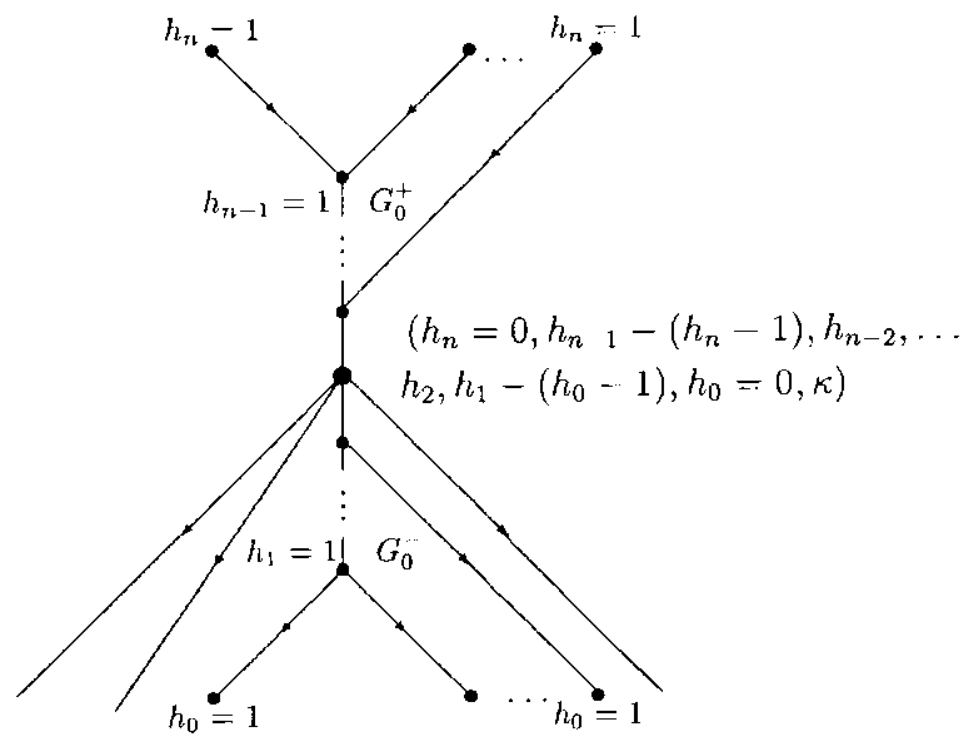

Figura 3.4: Explosäo parcial de um vértice repulsor.

Observe que substituindo os novos rótulos no vértice parcialmente explodido, isto é, $h_{n-1}$ por $h_{n} 1 \cdots\left(h_{n}-1\right), h_{1}$ por $h_{1}-\left(h_{0}-1\right)$, $e^{+}$por 1 e $e^{-}$por $e^{-}+1$, em (2.7), (2.10), (2.11) no caso de dimensāo ímpar ou (2.15), (2.16) no caso de dimensão par e sucessivamente até as desigualdades (2.12), (2.13) e (2.14), obtemos as desigualdades de Poincaré-Hopf gerais (2.17), (2.18) e (2.20). 


\subsubsection{Algoritmo da explosão de um vértice do tipo sela}

$\mathrm{Na}$ Seção 3.1.1, a explosão parcial de um vértice a um vértice do tipo sela foi estabelecida. Nesta seção continuaremos a denotar o vértice parcialmente explodido por $v$. Agora $v$ é um vértice do tipo sela rotulado com $\left(0, h_{1}, h_{2}, \ldots, h_{n-1}, 0, \kappa\right)$ e arestas incidentes positivamente rotuladas com $\left(\left(\beta_{0}^{+} ; \ldots, \beta_{n-1}^{+}\right)_{i}\right)_{i=1}^{e^{+}}$e arestas incidentes negativamente rotuladas $\operatorname{com}\left(\left(\beta_{0}^{-}, \ldots, \beta_{n-1}^{-}\right)_{i}\right)_{i=1}^{e^{-}}$, onde $i$ denota a aresta. Seja $B_{j}^{+}=\sum_{i=1}^{e^{+}}\left(\beta_{j}^{+}\right)_{i}$ e $B_{j}^{-}=\sum_{i=1}^{e^{-}}\left(\beta_{j}^{-}\right)_{i}$. Ver Figura 3.5. Observe que $B_{0}^{-}=e^{-}$e $B_{0}^{+} \div e^{+}$.

Esquematizamos o algoritmo que explode um vértice do tipo sela em quatro etapas básicas:

1. ajuste das arestas incidentes $\longrightarrow$ define $G^{+}$e $G^{-}$;

2. inserção de ciclos;

3. explosão linear fora das dimensões médias $\rightarrow$ define $G^{+} \cup \bigcup_{j=1}^{\ell} L_{j}^{+}$e $G^{-} \cup \bigcup_{j=1}^{\ell} L_{j}$ onde $\ell<$ dimensão média;

4. explosão da dimensão média $\longrightarrow$ consideramos $n$ ímpar, $n=0 \bmod 4, n=2 \bmod 4$.

A primeira parte do algoritmo consiste em ajustar as arestas incidentes do grafo. Para este propósito definimos dois grafos $G^{+}$e $G^{-}$. O grafo $G^{+}$tem a propriedade de ter $e^{+}$arestas incidentes positivamente e uma aresta incidente negativamente. Scrá formado usando apenas singularidades de índice $n-1$ do tipo $(n-1)$-d. O grafo $G^{-}$ tem a propriedade de ter $e^{-}$arestas incidentes negativamente e uma aresta incidente positivamente. Será formado usando apenas singularidades de índice 1 do tipo 0-c.

A segunda parte do algoritmo consiste em unir a aresta incidente negativamente de $G^{+}$ao grafo linear $L^{+}$formado por vértices do tipo Morse rotulados com $h_{n-j}=1$ e $j<$ dimensões médias. Analogamente, a aresta incidente positivamente de $G^{-}$será unida ao grafo linear $L^{-}$formado por vértices do tipo Morse rotulados com $h_{j}=1 \mathrm{e}$ $j<$ dimensōes médias. Relembre que a variação dos rótulos sobre as arestas é sempre considerarla na direção oposta a orientação do grafo.

A terceira parte do algoritmo consiste em inserir os ciclos. 
A quarta parte do algoritmo consiste em inserir singularidades na dimensão média, se existir, e unir $J^{+}$a $L^{-}$se estes já não tiverem sido unidos n passo anterior. Certamente, tudo isto é feito, de forma que os pesos sobre as duas últimas arestas a serem unidas coincidam.

Prosseguiremos descrevendo o algoritmo.

\section{Algoritmo}

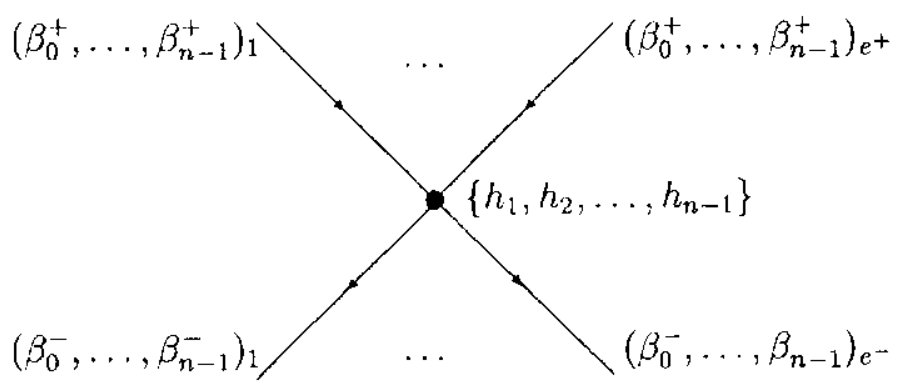

Figura 3.5: Vértice a ser explodido.

Ajuste das arestas incidentes: $G^{-}$e $G^{+}$.

Escolha $e^{-}-1$ vértices rotulados com $h_{1}=1$ do tipo $0-c$. Isto é possivel pela última desigualdade em (2.20). Escolhendo este número de vértices rotulados com 1singularidades, $G^{-}$é formado com $e^{-*}$ arestas incidentes negativamente e uma aresta incidente positivamente. Singularidades do tipo 0 -c nāo alteram $\beta_{i} \operatorname{com} 0<i<n-1$. Fste tipo de singularidade decresce $\beta_{0}$ e por dualidade $\beta_{n-1}$. Logo, a aresta incidente positivamente de $G$ tem $B_{0}^{-}=B_{r-1}^{-}=1$ e $B_{j}=\sum_{i=1}^{e^{-}}\left(\beta_{j}^{-}\right)_{i} \operatorname{com} j=\{1, \ldots, n-2\}$. Ver Figura 3.6.

Analogamente, o grafo $G^{+}$é formado escolhendo $e^{\dagger}-1$ vértices rotulados $h_{n-1}=1$ do tipo $(n-1)$-d. Este grafo terá $e^{+}$arestas incidentes positivamente e uma aresta incidente negativamente. Estas singularidades não alteram $\beta_{i}, 0<i<n-1$. A aresta incidente negativamente de $G^{+}$será rotulada $\operatorname{com} B_{0}^{+}=B_{n-1}^{+}=1$ e $B_{j}^{+}=\sum_{i=1}^{e^{+}}\left(\beta_{j}^{+}\right)_{i}$ com $j=\{1, \ldots, n-2\}$.

\section{Inserção de ciclos}




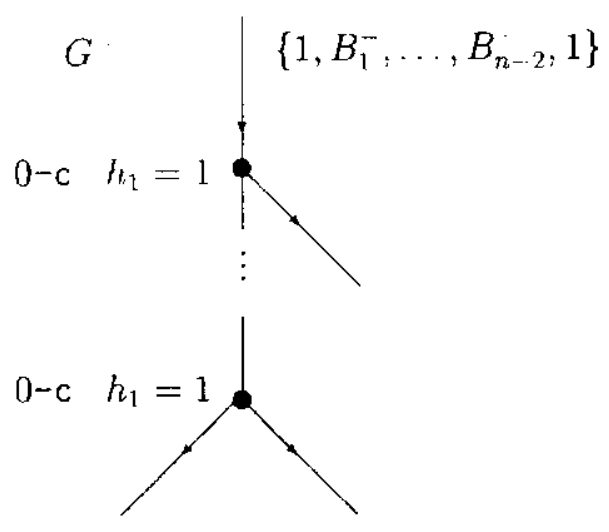

Figura 3.6: Explosão das arestas incidentes negativamente.

Um ciclo elementar é um par de $\left(h_{1}^{c}, h_{n-1}^{d}\right)$ com uma aresta rotulada com $(1,0, \ldots, 0,1)$ e outra aresta rotulada $\operatorname{com}\left(1, \beta_{1}, \ldots, \beta_{n-2}, 1\right)$. Ver Figura 3.7.

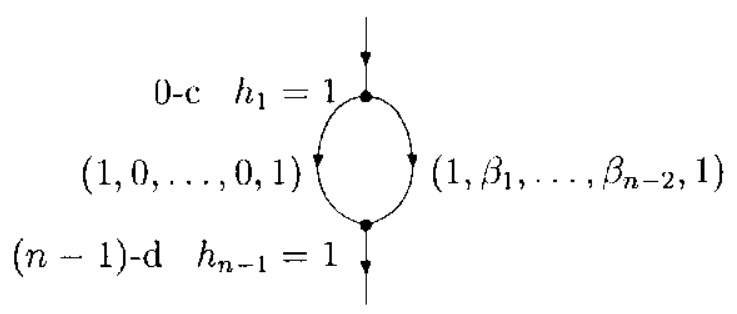

Figura 3.7: $\operatorname{Par}\left(h_{1}^{c}, h_{n-1}^{d}\right)$

Sem perda de generalidade, junte a $G^{-}$, $\kappa$ ciclos elementares onde $\left(1, \beta_{1}, \ldots, \beta_{n-2}, 1\right)=$ $\left(1, B_{1}^{-}, \ldots, B_{n-2}^{-}, 1\right)$. Naturalmente isto pode também ser feito a $G^{\dagger}$. É claro que uma vez que $\kappa$ ciclos são inseridos o número de vértices rotulados com $h_{1}=1$ do tipo 0-c é maior ou igual a $\kappa$. Analogamente, o número de vértices rotulados com $h_{n-1}=1$ do tipo $(n-1)-d$ é maior ou igual a $\kappa$.

Logo, inserimos $h_{1}^{c}=\kappa+e^{-}-1$ vértices rotulados com $h_{1}=1$ do tipo 0-c. Analogamente, inserimos $h_{n-1}^{d}=\kappa+e^{:}-1$ vértices rotulados com $h_{n-1}=1$ do tipo $(n-1)$-d. Isto é possível devido as duas últimas desigualdades em (2.20), que assegura que para este 
vértice do tipo sela $v\left(h_{0}=h_{n}=0\right)$ :

$$
\left\{\begin{array}{l}
h_{1}>-1+e^{-}+\kappa \\
h_{n-1}>-1+e^{+}+\kappa
\end{array}\right.
$$

O último ciclo inserido (veja Figura3.8) tem aresta incidente positivamente rotulada com

$$
\left(1, B_{1}^{-}, B_{2}^{-}, \ldots, B_{r i d}^{-}, \ldots, B_{n-2}^{-}, 1\right)
$$

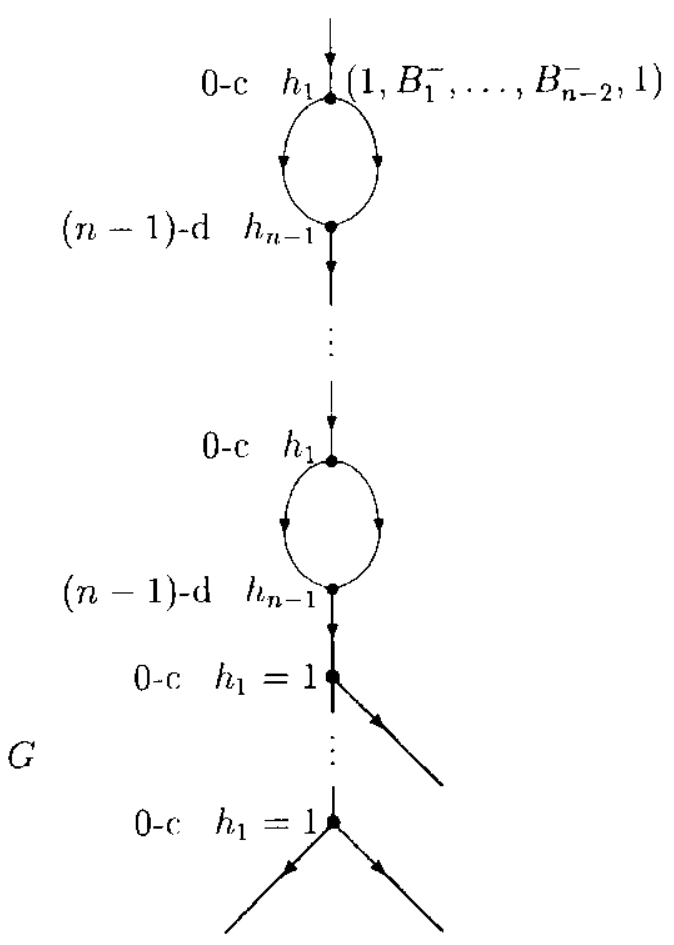

Figura 3.8: Arestas incidentes negativamente cxplodidas e ciclos inseridos.

A explosão linear fora das dimensões médias (Passo 1, .., Passo $\ell$ )

Passo 1 - $O$ ajuste de $B_{1}$ e por dualidade $B_{n-2}$.

O rótulo da aresta incidente positivamente a $G^{\prime \prime}$ é

$$
\left\{1, B_{1}^{-}, B_{2}, \ldots, B_{m i d}^{-}, \ldots, B_{n-2}^{-}, 1\right\}
$$

Para ajustar $B_{1}^{-}$e por dualidade $B_{n-2}^{-}$adicione um grafo linear $L_{1}^{-}$a $G^{-}$. Isto é feito inserindo $h_{1}^{d}$ vértices $h_{1}=1$ do tipo $1-\mathrm{d}$ a $G^{-}$. Logo, o rótulo sobre a aresta incidente 
positivamente ao último vértice deste tipo inserido será $B_{1}^{-}+h_{1}^{d}$. Observe que a inserção de vértices $h_{2}=1$ do tipo 1-c decresce $B_{1}^{-}$. Assim, após a inserção de $h_{2}^{c}$ vértices $h_{2}=1$ do tipo 1-c, $G^{-} \cup L_{1}^{-}$é formado. O rótulo sobre a aresta incidente positivamente a $G^{-} \cup L_{1}^{-}$ será $B_{1}^{-}+h_{1}^{d}-h_{2}^{c}$. Por dualidade o rótulo $B_{n-2}^{-}$foi modificado para $B_{n-2}^{-}+h_{1}^{d}-h_{2}^{c}$.

$O$ rótulo da aresta incidente negativamente a $G^{\dagger}$ é

$$
\left\{1, B_{1}^{+}, B_{2}^{+}, \ldots, B_{\text {mid }}^{+}, \ldots, B_{n-2}^{+}, 1\right\}
$$

Para ajustar $B_{1}^{+}$e por dualidade $B_{n-2}^{\dagger}$ adicione um grafo linear $L_{1}^{+}$a $G^{+}$. Isto é feito inserindo $h_{n-1}^{c}$ vértices $h_{n-1}=1$ do tipo $(n-2)$-c. Após a inserção destes vértices o rótulo sobre a aresta incidente negativamente ao último vértice deste tipo será $B_{n-2}^{\dagger}+h_{n-1}^{c}$. Subseqüentemente, adicione $h_{n-2}^{d}$ vértices $h_{n-2}=1$ do tipo $(n-2)$-d formando $G^{+} \cup$ $L_{1}^{+}$. Após a inserçào destes vértices o rótulo sobre as arestas incidentes negativamente a $G^{+} \cup L_{1}^{+}$será $B_{n-2}^{+}-h_{n}^{d}{ }_{2}+h_{n}^{c}$. Por dualidade, o rótulo $B_{1}^{+}$foi modificado para $B_{1}^{\dagger}-h_{n-2}^{d}+h_{r-1}^{c}$.

Como a inserção de qualquer outro tipo de vértice não altera o primeiro e o $(n-2)$ ésimo número de Betti é necessário que

$$
B_{1}=B_{1}^{-}+h_{1}^{d}-h_{2}^{c}=B_{1}^{+}-h_{n-2}^{d}+h_{n-1}^{c} .
$$

Se a igualdade acima é verdadeira então por dualidade temos a seguinte desigualdade:

$$
B_{n-2}=B_{n 2}+h_{1}^{d}-h_{2}^{c}=B_{n 2}^{+}+h_{n-2}^{d}-h_{n-1}^{c}
$$

Assim os rótulos de $B_{1}$ e $B_{n-2}$ foram ajustados. A aresta incidente positivamente a $G^{-} \cup L_{1}^{*}$ tem os seguintes rótulos:

$$
\left\{1, B_{1}, B_{2}^{-}, \ldots, B_{\ell}^{-}, \ldots, B_{m i d}^{-}, \ldots, B_{n-\ell-1}^{-}, \ldots, B_{n-3}^{-}, B_{n-2}, 1\right\}
$$

e da aresta incidente negativamente a $G^{+} \cup L_{1}^{+}$tem os seguintes rótulos:

$$
\left\{1, B_{1}, B_{2}^{+}, \ldots, B_{\ell}^{+}, \ldots, B_{m i d}^{+}, \ldots, B_{n-\ell-1}^{+}, \ldots, B_{r,-3}^{+}, B_{n-2}, 1\right\}
$$

Este processo de ajustar $B_{\ell}^{+}$e $B_{n-\ell-1}^{+}$será repetido em ordem crescente exceto nas dimensões médias. O processo geral será representado no próximo passo.

Passo $\ell$ - $O$ ajuste de $B_{\ell}$ e por dualidade $B_{n-\ell-1}$ 


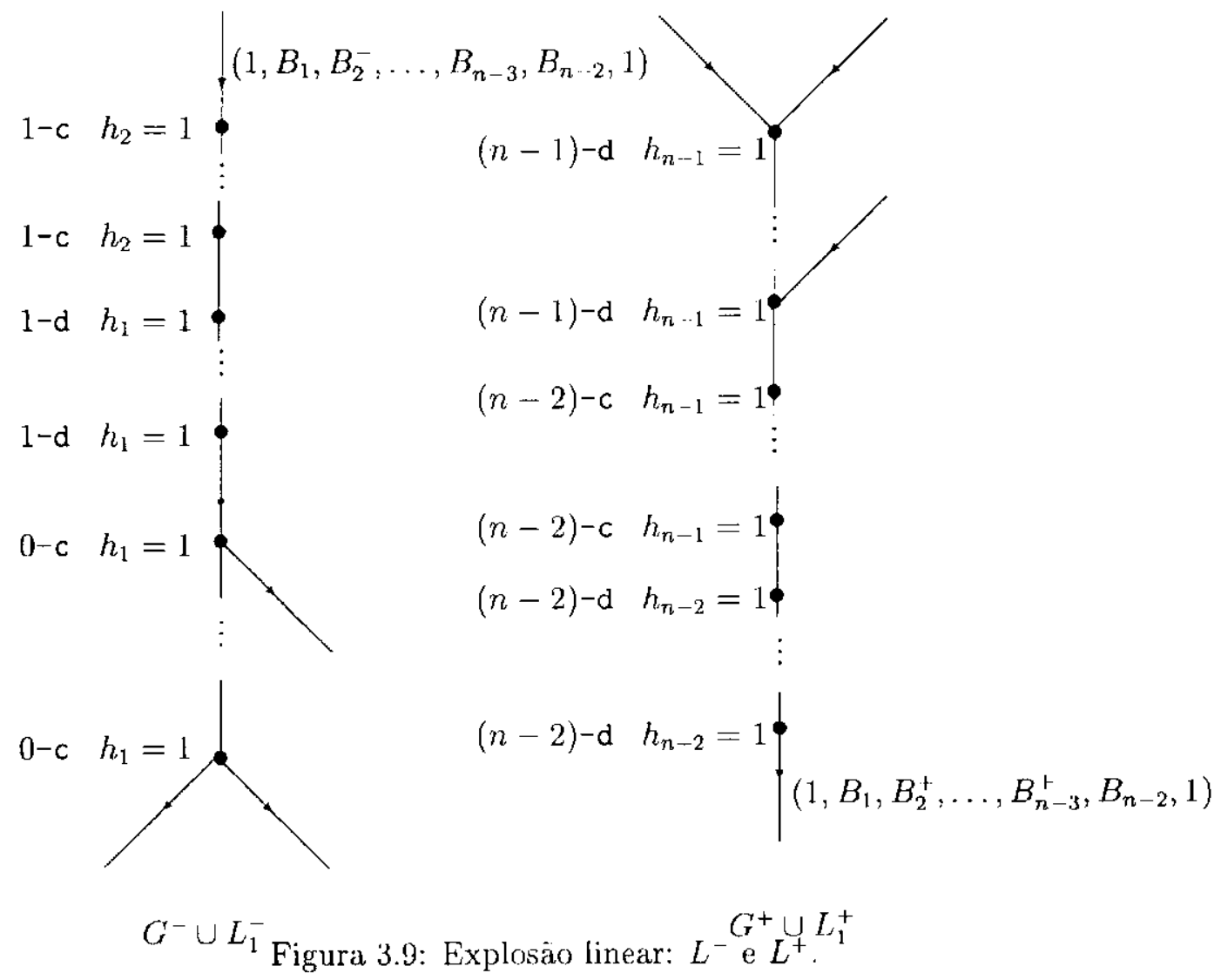

Neste ponto, assumiremos por indução que os ajustes de $B_{j}$ for $j<\ell$ e por dualidade $B_{n-j-1}$ for $j<\ell$ foram feitos em ordem crescente para $j$. Logo, vários grafos lineares já foram adicionados a $G^{+}$formando neste ponto um grafo $G^{+} \cup \bigcup_{i=1}^{\ell-1} L_{i}^{+}$cuja aresta incidente negativamente ao último vértice inserido está rotulada com

$$
\left\{1, B_{1}, \ldots, B_{\ell-1}, B_{\ell}^{+}, \ldots, B_{m i d}^{+}, \ldots, B_{n-\ell-1}^{+}, B_{n-\ell}, \ldots, B_{n_{-}-2}, 1\right\}
$$

Analogamente, vários grafos lineares foram adicionados a $G$ formando neste ponto um grafo linear $G^{-} \cup \bigcup_{i=1}^{\ell-1} L_{i}^{-}$cuja aresta incidente positivamente ao último vértice inserido está rotulada com

$$
\left\{1, B_{1}, \ldots, B_{\ell-1}, B_{\ell}^{-}, \ldots, B_{m i d}^{-}, \ldots, B_{n-\ell-1}^{-}, B_{n-\ell}, \ldots, B_{n-2}, 1\right\}
$$

Para ajustar $B_{\ell}$ e por dualidade $B_{n-\ell-1}$ insira $h_{\ell}^{d}$ vértices $h_{\ell}=1$ do tipo $\ell$-d a $G \cup$ $\bigcup_{i=1}^{\ell-1} L_{i}$. Logo, o rótulo sobre a aresta incidente positivamente do último vértice deste tipo inserido será $B_{\ell}^{-}+h_{\ell}^{d}$. Observe que a inserção de vértices do tipo $\ell$-c decresce $B_{\ell}^{-}$. Assirn, 
após a inserção de $h_{\ell+1}^{c}$ vértices $h_{\ell+1}=1$ do tipo $\ell$-c o rótulo sobre a aresta incidente positivamente a $G^{-} \cup \bigcup_{i=1}^{\ell} L_{i}^{-}$será $B_{\ell}+h_{\ell}^{d}-h_{\ell+1}^{c}$. Por dualidade o rótulo $B_{n-\ell-1}^{-}$foi modificado para $B_{n-\ell-1}^{-}+h_{\ell}^{d}-h_{\ell+1}^{c}$.

Analogamente, a inserção de $h_{n-\ell}^{c}$ vértices $h_{n-\ell}=1$ do tipo $(n-\ell-1)$-c produzirá uma aresta incidente positivamente rotulada com $B_{n \ell-1}^{+}+h_{n-\ell}^{c}$. Subsequientemente a inserção de $h_{n-\ell-1}^{d}$ vértices $h_{n-\ell-1}=1$ do tipo $(n-\ell-1)$-d formará $G^{+} \cup \bigcup_{i=1}^{\ell} L_{i}^{+}$e os rótulos sobre a aresta incidente negativamente é $B_{n-\ell-1}^{+}-h_{n-\ell-1}^{d}+h_{n-\ell}^{c}$. Por dualidade o rótulo $B_{\ell}^{+}$foi modificado para $B_{\ell}^{+}-h_{n-\ell-1}^{d}+h_{n}^{c} \ell$.

Como a inserção de qualquer outro tipo de vértice não altera o $\ell$-ésimo e o $(n-\ell-1)$ ésimo número de Betti é necessário que

$$
B_{\ell}=B_{\ell}^{-}+h_{\ell}^{d}-h_{\ell+1}^{c}=B_{\ell}^{+}-h_{n-\ell-1}^{d}+h_{n-\ell}^{c}
$$

Se a igualdade acima for verdadeira então por dualidade temos a seguinte igualdade:

$$
B_{n-\ell-1}=B_{n-\ell-1}-h_{\ell}^{d}-h_{\ell+1}^{c}=B_{n-\ell \cdot 1}^{+}-h_{n-\ell-1}^{d}+h_{n-\ell}^{c} .
$$

Os rótulos de $B_{\ell}$ e $B_{n-\ell-1}$ para $0<\ell<$ mid foram todos ajustados. Resta ajustar os rótulos da dimensão média. Isto será feito no próximo passo.

\section{Explosão da dimensão média}

Neste ponto os ajustes dos rótulos nas dimensões médias devem ser feitos. É necessário considerar o caso quando $n-1$ é par e logo existe apenas um rótulo na dimensão média $B_{\frac{n-1}{2}}$ e o caso quando $n-1$ é ímpar e existem dois rótulos na dimensão média.

Caso $n-1$ par

Seja $n=2 i+1$. Neste ponto existem dois grafos $G^{-} \cup \bigcup_{j=1}^{i-1} L_{j}^{-j}$ com aresta incidente positivamente rotulada com $\left\{1, B_{1}, B_{2}, \ldots, B_{i-1}, B_{i}^{-}, B_{i+1}, \ldots, B_{2 i-1}, 1\right\}$ e $G^{+} \cup \bigcup_{j=1}^{i-1} L_{j}^{+} \operatorname{com}$ aresta incidente negativamente rotulada $\operatorname{com}\left\{1, B_{1}, B_{2} \ldots, B_{i \cdots 1}, B_{i}^{+}, B_{i+1}, \ldots, B_{2 i-1}, 1\right\}$.

Para ajustar $B_{i}^{-}$insira $h_{i}^{d}$ vértices $h_{i}=1$ do tipo $i$-d na aresta incidente positivamente a $G^{-} \cup \bigcup_{j=1}^{i-1} L_{j}^{-}$. Logo, o rótulo sobre a aresta incidente positivamente ao último vérticé inserido deste tipo é $B_{i}^{-}+2 h_{i}^{d}$. 
Assim, após a inserção de $h_{i+1}^{c}$ vértices $h_{i+1}=1$ do tipo $i$-c na aresta incidente negativamente a $G^{+} \cup \bigcup_{j=1}^{i-1} L_{j}^{+}$, o rótulo sobre a aresta incidente negativamente do último vértice inserido deste tipo é $B_{i}^{+}+2 h_{i+1}^{c}$.

Mais ainda, é necessário que

$$
B_{i}=B_{i}^{-}+2 h_{i}^{\prime}=B_{i}^{+}+2 h_{i+1}^{c}
$$

Como, os rótulos sobre a aresta incidente negativamente de um dos grafos agora coincide inteiramente com os rótulos sobre a aresta incidente positivamente do outro grafo, eles podem ser unidos formando um grafo conexo.

\section{Sistema $h_{\kappa}^{c d}$}

Logo, no final deste ajuste, (3.2), (3.3), (3.4), o seguinte sistema linear deve ser resolvido para $\left(h_{1}^{c}, h_{1}^{d}, \ldots, h_{2 i}^{r}, h_{2 i}^{d}, \kappa\right)$, para que o algoritmo funcione.

$$
\left\{\begin{array}{l}
e^{--}-1-h_{1}^{c}+\kappa=0 \\
\left\{h_{j}=h_{j}^{c}+h_{j}^{d}, j=1, \ldots, 2 i\right. \\
e^{+}-1-h_{2 i}^{d}+\kappa=0 \\
\left\{\begin{array}{l}
-\left(B_{1}^{+}-B_{1}^{-}\right)+h_{1}^{d}-h_{2}^{c}-h_{2 i}^{c}+h_{2 i-1}^{d}=0 \\
-\left(B_{2}^{+}-B_{2}^{-}\right)+h_{2}^{d}-h_{3}^{c}-h_{2 i-1}^{c}+h_{2 i-2}^{d}=0 \\
\vdots \\
\frac{-\left(B_{i}^{+}-B_{i}^{-}\right)}{2}+h_{i}^{d}-h_{i+1}^{c}=0
\end{array}\right.
\end{array}\right.
$$

Caso $n-1$ ímpar.

Seja $n=2 i$. Existem dois casos a considerar:

Caso 1: $n=0 \bmod 4$.

Observe que neste caso a variação do número de Betti na dimensão média $B_{i-1}^{-}$e seu dual $B_{i}^{-}$varia por 0 se o vértice é do tipo $\beta$-i ou por 1 caso contrário.

Insira $h_{i-1}^{d}$ vértices $h_{i-1}=1$ do tipo $(i-1)$-d na aresta incidente negativamente a $G^{-} \cup \bigcup_{j=1}^{i-2} L_{j}^{-}$. Logo, o rótulo sobre a aresta incidente positivamente ao último vértice deste tipo será $B_{i-1}^{-}+h_{i+1}^{d}$. Subseqüentemente, insira $h_{i}^{c}$ vértices $h_{i}=1$ do tipo $(i-1)$-c. O rótulo sobre a aresta incidente positivamente a $G^{-} \cup \bigcup_{j-1}^{i-1} L_{j}^{-}$será $B_{i-1}+h_{i-1}^{d}-h_{i}^{c}$. Por dualidade o rótulo $B_{i}^{-}$será modificado para $B_{i}^{-}+h_{i-1}^{d}-h_{i}^{c}$. 
Analogamente, insira $h_{i+1}^{c}$ vértices $h_{i+1}=1$ do tipo $i$-c na aresta incidente negativamente a $G^{+} \cup \bigcup_{j=1}^{i-2} L_{j}^{+}$. Logo, o rótulo sobre a aresta incidente negativamente ao último vértice deste tipo inserido será $B_{i}^{+}+h_{i+1}^{c}$. Subseqüentemente, insira $h_{i}^{d t}$ vértices $h_{i}=1$ do tipo $i$-d formando $G^{+} \cup \bigcup_{j=1}^{i-1} L_{j}^{+}$e o rótulo sobre a aresta incidente negativamente a este grafo será $B_{i}^{+}-h_{i}^{d}+h_{i+1}^{c}$. Por dualidade o rótulo $B_{i-1}^{+}$foi modificado para $B_{i-1}^{+}-h_{i}^{d}+h_{i+1}^{c}$.

Mais ainda, é necessário que

$$
B_{i-1}^{-}+h_{i \cdots 1}^{d}-h_{i}^{c}=B_{i-1}^{+}-h_{i}^{d}+h_{i+1}^{c} .
$$

Por dualidade se temos a equação acima então a seguinte é verdadeira

$$
B_{i}+h_{i 1}^{d}-h_{i}^{c}=B_{i}^{+}-h_{i}^{d}+h_{i+1}^{c} \text {. }
$$

Como os rótulos da aresta incidente negativamente a um dos grafos agora coincide inteiramente com os rótulos da aresta incidente positivamente ao outro grafo, estes podem se unidos para formar um grafo conexo.

\section{Sistema $h_{\kappa}^{c d}$}

Logo, no final deste ajuste, (3.2), (3.3), (3.6), ficamos com o seguinte sistema linear que deve ser resolvido para $\left(h_{1}^{c}, h_{1}^{d}, \ldots, \beta^{i}, \ldots, h_{2 i-1}^{c}, h_{2 i-1}^{d}, \kappa\right)$ que o algoritmo funcione.

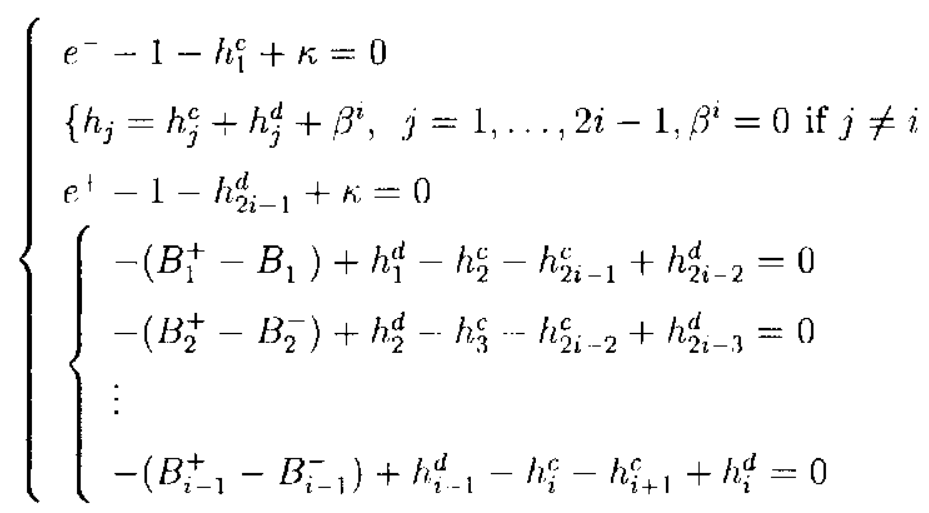

Caso 2: $n=2 \bmod 4$.

Os ajustes neste caso são idênticos ao caso anterior exceto pelo fato que não existem vértices do tipo $\beta$-i. 
Neste caso o sistema linear que deve ser resolvido para $\left(h_{1}^{c}, h_{1}^{d}, \ldots, h_{2 i-1}^{c}, h_{2 i-1}^{d}, \kappa_{i}\right)$ é:

$$
\left\{\begin{array}{l}
e^{-}-1-h_{1}^{c}+\kappa=0 \\
\left\{h_{j}=h_{j}^{c}+h_{j}^{d}, j=1, \ldots, 2 i-1\right. \\
e^{+}-1-h_{2 i-1}^{d}+\kappa=0 \\
\left\{\begin{array}{l}
-\left(B_{1}^{+}-B_{1}^{-}\right)+h_{1}^{d}-h_{2}^{c}-h_{2 i-1}^{c}+h_{2 i-2}^{d}=0 \\
-\left(B_{2}^{+}-B_{2}^{-}\right)+h_{2}^{d}-h_{3}^{c}-h_{2 i-2}^{c}+h_{2 i-3}^{d}=0 \\
\vdots \\
-\left(B_{i-1}^{+}-B_{i-1}^{-}\right)+h_{i}^{d}-h_{i}^{c}-h_{i+1}^{c}+h_{i}^{d}=0
\end{array}\right.
\end{array}\right.
$$

O vértice poderá ser explodido se o sistema $h_{\kappa}^{c d}$ possui solução. As hipóteses do Teorema 3.0.1 afirmam que o vértice satisfaz as desigualdades de Poincaré-Hopf (2.10), (2.11) e sucessivamente até as desigualdades (2.12), (2.13) e (2.14) no caso ímpar, e desigualdades (2.15) no caso par. Mostramos na Seção 3.2 que o sistema $h_{\kappa}^{c d}$ tern soluções inteiras não-negativas se e somente se as desigualdades de Poincaré-Hopf estiverem satisfeitas. A lém disso, o número de explosões que um dado vértice pode ter ou equivalentemente o número de soluções não-negativas que o sistema $h_{\kappa}^{c d}$ possui, é também determinado.

\subsubsection{Algoritmo de Explosão para $n=2$}

Seja $v$ um vértice do tipo sela generalizada com $\left\{h_{0}, h_{1}, h_{2}\right\}$ e com $e^{+}$arestas incidentes positivamente $\mathrm{e} e^{-}$arestas incidentes negativamente.

Como nos casos anteriores o primeiro passo no algoritmo realizará a explosão parcial como na Subseção 3.1.1 para obter um vértice do tipo sela com $\left\{h_{0}=0, h_{1}-\left(\left(h_{2}-1\right)+\left(h_{0}-\right.\right.\right.$ 1)), $\left.h_{2}=0\right\}$. Esta parte do algoritmo usa (2.19) para garantir que $h_{1}-\left(\left(h_{2}-1\right)+\left(h_{0}-1\right)\right) \geq$ $\left(e^{+}+e^{-}\right) \geq 0$. Nesta explosão parcial o número de arestas incidentes positivamente e negativamente aumentou por um.

O próximo passo será o que ajusta as arestas incidentes. Em outras palavras, usaremos $\left(\left(e^{+}+1\right)-1\right)$ vértices rotulados com $h_{1}=1$ do tipo 1 -d e $\left(\left(e^{-}+1\right)-1\right)$ vértices rotulados com $h_{1}=1$ do tipo 0-c. Este passo está garantido pela desigualdade de Poincaré-Hopf (2.19) que assegura que $h_{1}-\left(\left(h_{2}-1\right)+\left(h_{0}-1\right)+\left(e^{r}+e^{-}\right)\right) \geq 0$.

Seja $\kappa=h_{1}-\left(\left(h_{2}-1\right)+\left(h_{0}-1\right)+\left(e^{+}+e^{-}\right)\right)$. Se $\kappa=0$ a explosão foi completada. Se 
$\kappa>0$ a condição que requer que $\kappa$ seja $\operatorname{par}^{1}$ nos permite concluir a explosão $\mathrm{com}$ pares de $\left\{h_{1}^{d}, h_{1}^{c}\right\}$ formando $\frac{\kappa}{2}$ ciclos. Obviamente esta explosão é única e representa um fluxo sobre um bloco orientável de gênero $g_{v}=\frac{\kappa}{2} \operatorname{com} e^{+}$componentes de bordo de entrada $\mathrm{e}$ e componentes de bordo de saída.

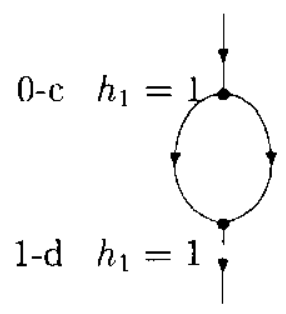

Figura 3.10: $\operatorname{Par}\left\{h_{1}^{d}, h_{1}^{c}\right\}$

\subsection{Soluções para os Sistemas Lineares}

Existem quatro possibilidades para $n$, a saber $n=0,1,2$ ou $3 \bmod 4$. A segunda e quarta possibilidades correspondem ao caso $n$ ímpar. Este caso é considerado na Subseção 3.2.1. A primeira e terceira possibilidades são analisadas nas Subseções 3.2 .2 e 3.2 .3 , respectivamente.

\subsubsection{Caso $n$ ímpar}

$\mathrm{O}$ sistema lincar

Assuma $n \geq 3, n=2 i+1$. Queremos encontrar uma solução inteira não negativa do sistema linear

\footnotetext{
'Se é ímpar a explosão pode ainda ser completada e representará um fluxo en um bloco não orientável.
} 


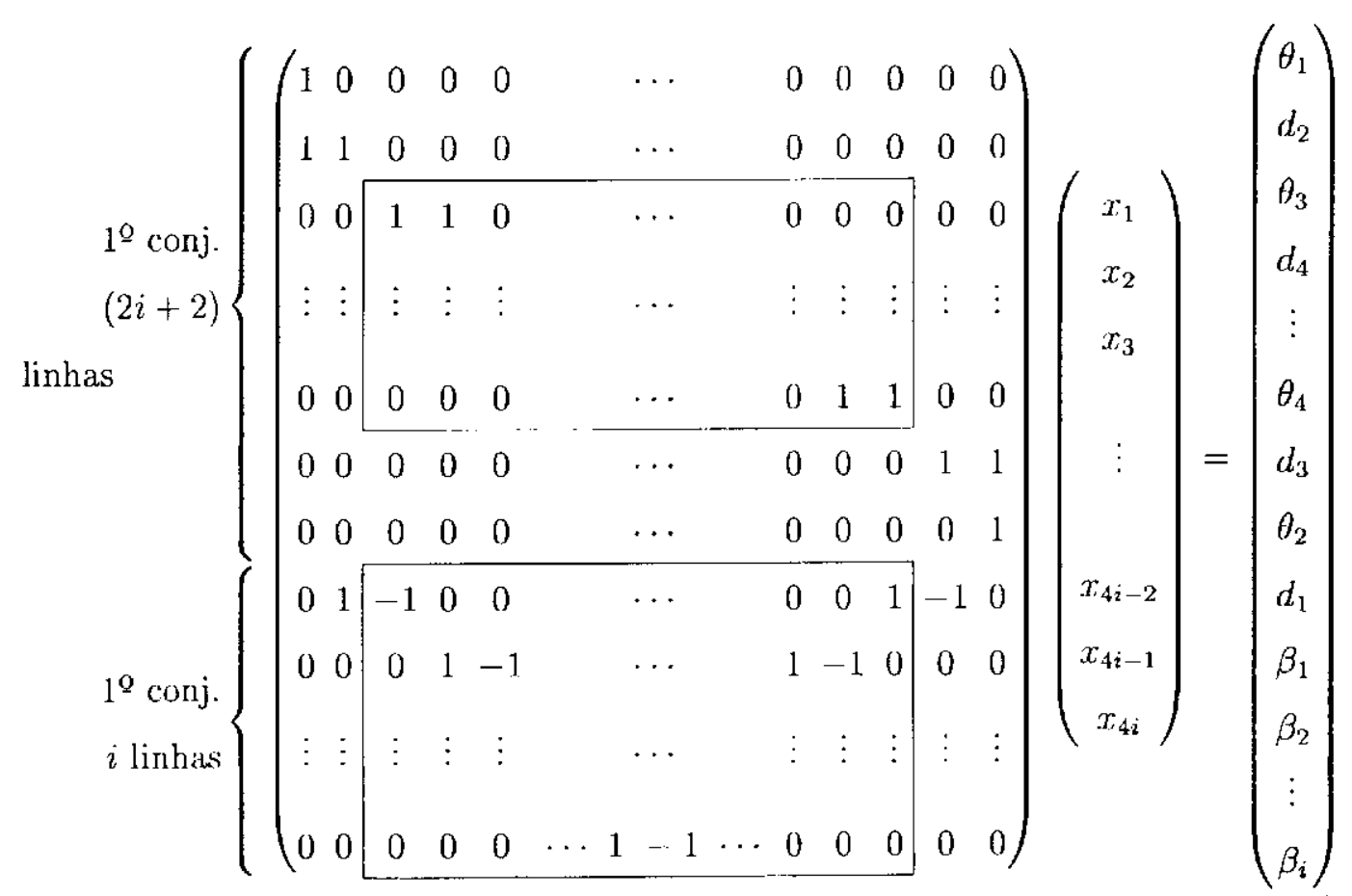

onde $\theta_{j}$ e $d_{j}$, para $j=1, \ldots, i+1$, são inteiros positivos e $\beta_{j}$, para $j=1, \ldots, i$, são inteiros. As equaçōes em (3.9) são divididas em dois conjuntos, o primeiro contendo $2 i+2$ equaçōes e o seguinte contendo $i$ equações, respectivamente. As linhas da matriz dos coeficientes $\bar{A}$ de (3.9) e seu vetor do lado direito $\bar{b}$ herdarn esta partição. As fórmulas para a j-ésima linha do primeiro grupo e o elemento correspondente do lado direito são

$$
\bar{A}_{j}= \begin{cases}e_{1}, & \text { se } j=1 \\ \boldsymbol{e}_{2 j-3}+e_{2 j-2}, & \text { se } 2 \leq j<2 i+1 \\ \boldsymbol{e}_{4 i}, & \text { se } j=2 i+2\end{cases}
$$

e

$$
\bar{b}_{j}=\left\{\begin{array}{ll}
\theta_{j}, & \text { se } 1 \leq j \leq i+1, j \text { impar } \\
d_{j}, & \text { se } 1<j \leq i+1, j \text { par } \\
\theta_{2 i+3-j}, & \text { se } i+1<j \leq 2 i+2, j \text { impar } \\
d_{2 i+3-j}, & \text { se } i+1<j \leq 2 i+2, j \text { par }
\end{array},\right.
$$


onde $\boldsymbol{e}_{j}$ é o $j$-ésimo vetor da base canônica do $\mathbb{R}^{4 i}$. No segundo grupo, a $j$-ésima linha e respectivo elemento do lado direito são como segue

$$
\bar{A}_{2 i+2+j}= \begin{cases}\boldsymbol{e}_{2 j}-\boldsymbol{e}_{2 j+1}+\boldsymbol{e}_{4 i-2 j}-\boldsymbol{e}_{4 i-2 j+1}, & \text { se } 1 \leq j \leq i-1 \\ \boldsymbol{e}_{2 i}-\boldsymbol{e}_{2 i+1}, & \text { se } j=i\end{cases}
$$

e $\bar{b}_{2 i+2 \nmid j}=\beta_{j}$, se $1<j<i$.

Quatro variáveis em (3.9) têm valores inteiros únicos que dependem de certos elementos do lado direito. Logo, a condiçāo de não negatividade da solução se traduz em um conjunto de desigualdades que estes sistemas devem satisfazer. Eliminando estas variáveis ficamos com 1 m sistema reduzido nas variáveis restantes as caixas destacadas em (3.9) contém a matriz de coeficientes deste sitema reduzido. Na Subseção 7 mostramos que a matriz de coeficientes deste novo sistema é totalmente unimodular ${ }^{2}$. Este fato, juntamente com a integralidade do lado direito, implica que se o sistema tem uma solução não negativa então ele terá uma solução inteira não negativa. L'm conjunto de restrições que é equivalente à existência de soluções não negativas para o sistema reduzido é construído na Subseção 7.

As seguintes equações podem se usadas para eliminar algumas variáveis em (3.9):

1. A primeira equação implica que $x_{1}=\theta_{1}$.

2. A primeira e a segunda equação implicam que $x_{2}=d_{2}-\theta_{1}$.

3. A $(2 i+2)$-ésima equação implica que $x_{4 i}=d_{1}$.

4. $\Lambda(2 i+2)$-ésima e $(2 i+1)$-ésima equações implicam que $x_{4 i-1}=\theta_{2}-d_{1}$.

Portanto, usando o fato que os vetores $\theta$, d e $\beta$ são inteiros, as seguintes condições são necessárias e suficientes para a solução parcial $\left(x_{1}, x_{2}, x_{4 i}, x_{4 i-1}\right)$ ser uma 4-upla inteira não negativa:

$$
\left\{\begin{array}{l}
d_{2}-\theta_{1} \geq 0 \\
\theta_{2}-d_{1} \geq 0
\end{array}\right.
$$

Tendo tratado as variáveis $x_{1}, x_{2}, x_{4 i-1}$ e $x_{4 i}$, passaremos a considerar o sistema reduzido anteriormente mencionado. Resta obter condiçōes necessárias e suficientes para a existência de soluçöes inteiras năo negativas deste último. Note, contudo, que quando

\footnotetext{
${ }^{2}$ Estas matrizes foram consideradas por Poincaré [18], 1900.
} 
$n=3$, ou, equivalentemente, $i=1$, nāo existe sistema reduzido, já que o sistema original contém apenas quatro incógnitas $x_{1}, x_{2}, x_{3}$ e $x_{4}$, tendo, portanto, uma única solução.

\section{Unimodularidade total da matriz dos coeficientes do sistema reduzido}

Chegamos no sistema reduzido (3.11) abaixo após eliminar equaçōes $1,2,2 i+1$ e $2 i+2$ e variáveis $x_{1}, x_{2}, x_{4 i-1}$ e $x_{4 i}$ em (3.9):

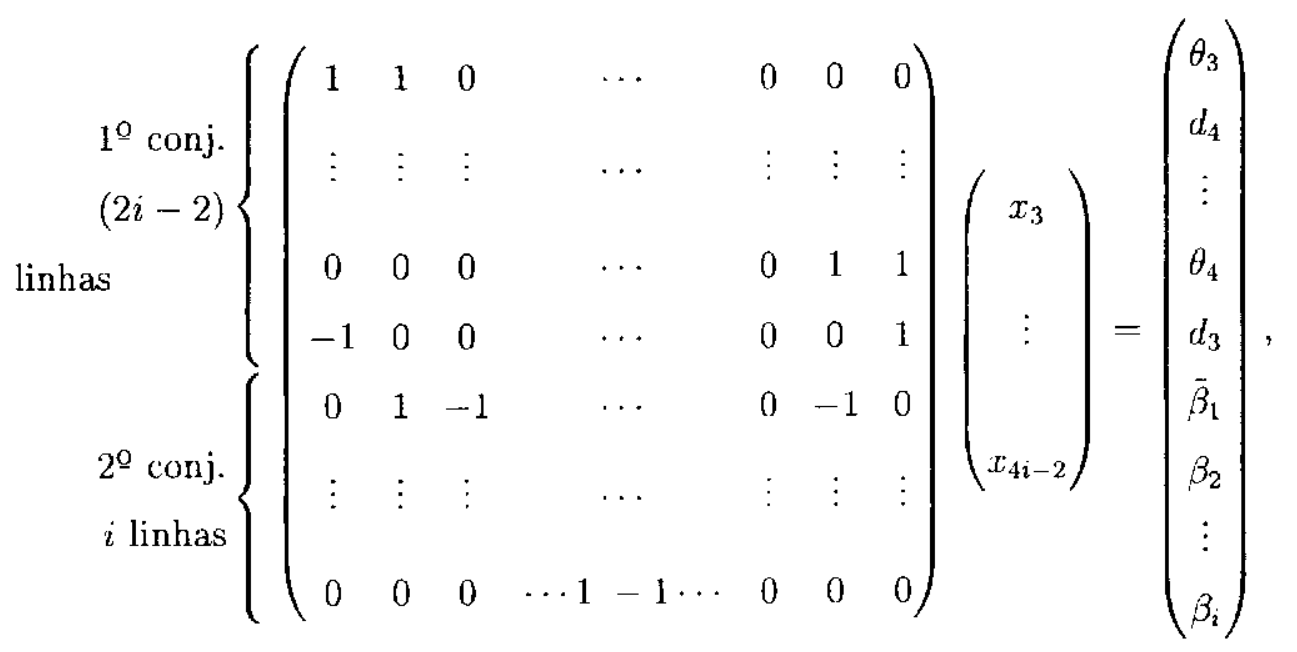

onde $\bar{\beta}_{1}=\beta_{1}-x_{2}+x_{4 i \cdot 1}=\beta_{1}+\theta_{1}+\theta_{2}-d_{1}-d_{2}$. Este sistema reduzido tem $3 i-2$ equações em $4 i-4$ incógnitas.

As linhas de $\overline{\bar{A}}$ e $\bar{b}$, a matriz de coeficientes e o lado direito do vetor de (3.11), herdam a partição estabelecida para para suas correspondentes em (3.9). Logo, o primeiro conjunto tem $2 i-2$ linhas/elementos (quatro equações foram eliminadas) enquanto o segundo conjunto mantém suas $i$ linhas/elementos. As fórmulas para a $j$-ésima linha e elementos do lado direito devem ser atualizadas de modo a incorporar a eliminação de linhas e colunas (equações e variáveis). No primeiro grupo temos

$$
\overline{\bar{A}}_{j}=\boldsymbol{e}_{2 j \cdots 1}+\boldsymbol{e}_{2 j}, \text { se } 1 \leq j \leq 2 i-2
$$

e

$$
\overline{\bar{b}}_{j}= \begin{cases}\theta_{j+2}, & \text { se } 1 \leq j \leq i-1, j \text { ímpar } \\ d_{j+2}, & \text { se } 1<j \leq i-1, j \text { par } \\ \theta_{2 i+1-j}, & \text { se } i-1<j \leq 2 i-2, j \text { ímpar } \\ d_{2 i+1-j}, & \text { se } i-1<j \leq 2 i-2, j \text { par }\end{cases}
$$


e, no segundo conjunto,

$$
\overline{\bar{A}}_{2 i-2+j}= \begin{cases}-e_{1}+e_{4 i-4}, & \text { se } j=1 \\ e_{2 j-2}-e_{2 j-1}+e_{4 i-2-2 j}-e_{4 i-1-2 j}, & \text { se } 2 \leq j<i-1 \\ e_{2 i-2}-e_{2 i-1}, & \text { se } j=i\end{cases}
$$

enquanto que $\overline{\bar{b}}_{2 i-1}=\bar{\beta}_{1}$ e $\overline{\vec{b}}_{2 i-2+j}=\beta_{j}$, para $j=2, \ldots, i$. Note que $\boldsymbol{e}_{\ell}$ é agora o $\ell$-ésimo vetor da base canonica do $\mathbb{R}^{4 i-4}$. O seguinte diagrama em blocos ajuda a compreender a estrutura de $\bar{A}$ :

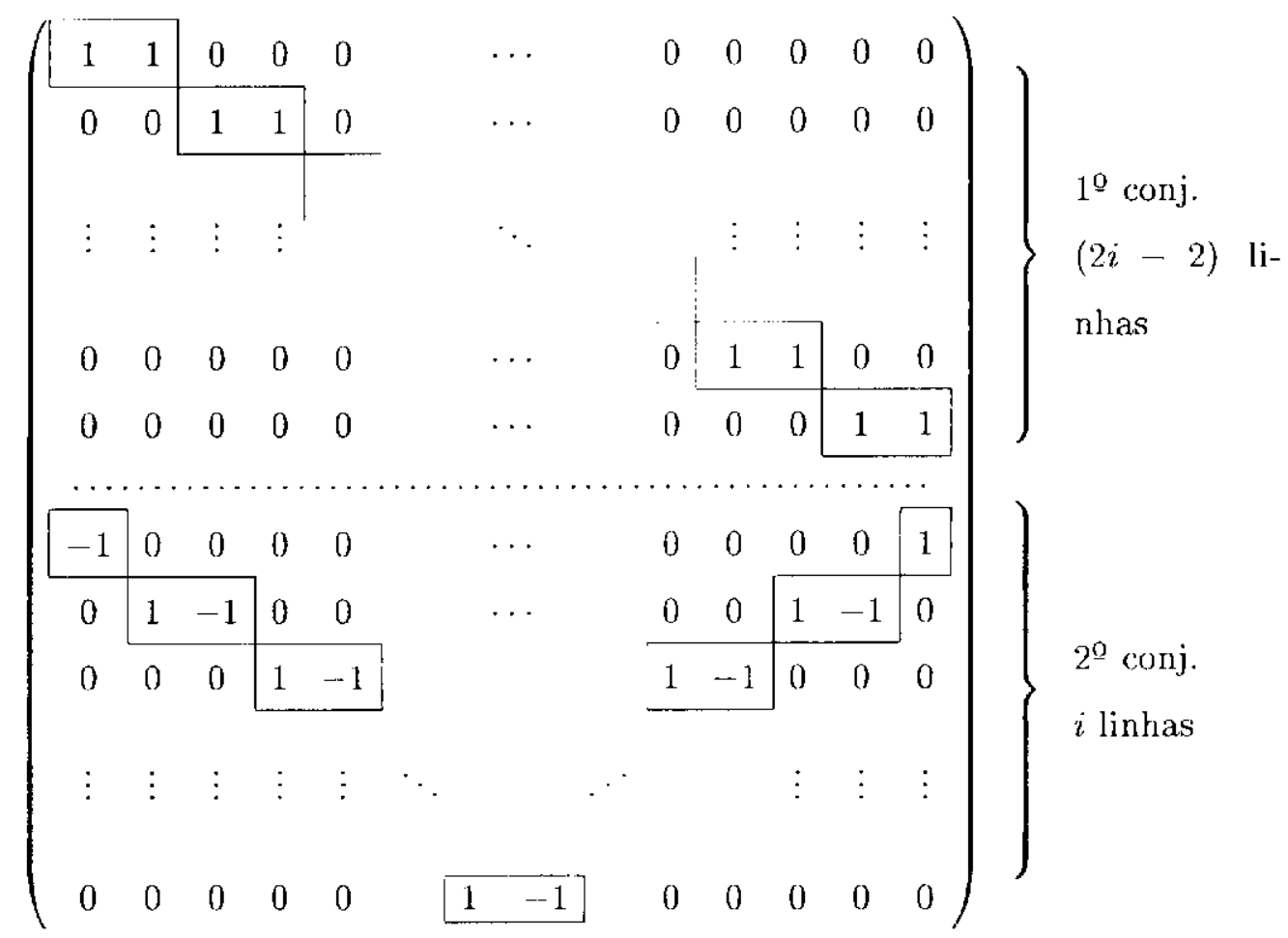

O primeiro conjunto de linhas forma uma matriz diagonal em blocos enquanto no segundo conjunto existern dois blocos por linha, posicionados simetricamente com relação ao meio.

Lema 3.2.1 Cada coluna de $\overline{\bar{A}}$ contém exatamente dois coeficientes näo nulos, um no primeiro conjunto de linhas e o outro no segundo conjunto.

Demonstração: Cada entrada em $\overline{\bar{A}}$ pertence a $\{0,1,-1\}$. Assim é suficiente mostrar que cada coluna de $\bar{A}$, a matriz obtida de $\overline{\bar{A}}$ substituindo cada entrada pelo seu valor absoluto, contém dois 1's, um em cada conjunto de linhas. 
Para tanto calculamos a soma das linhas no primeiro e segundo conjunto de $\tilde{A}$ :

1. Somando as linhas no primeiro conjunto, isto é, linhas 1 a $2 i-2$ de $\tilde{A}$ :

$$
\begin{aligned}
& \sum_{j=1}^{2 i-2} \tilde{A}_{j}=\sum_{j=1}^{2 i-2} \overline{\bar{A}}_{j}=\sum_{j=1}^{2 i-2}\left(\boldsymbol{e}_{2 j-1}+e_{2 j}\right) \\
& =\sum_{\ell \in\{1, \ldots, 4 i-4\}} e_{\ell}+\sum_{\ell \in\{1, \ldots, 4 i \cdots 4\}} e_{\ell} \\
& \ell \text { impar } \ell \text { par } \\
& =\sum_{\ell=1}^{4 i-4} e_{\ell}=\mathbf{1}_{4 i-4},
\end{aligned}
$$

onde $\mathbf{1}_{m}$ denota o vetor de uns em $\mathbb{R}^{m}$. Portanto cada coluna de $\tilde{A}$ contém exatamente uma entrada não nula no primeiro conjunto de linhas, ou seja, 1.

2. Somando as $i$ linhas no segundo conjunto:

$$
\begin{aligned}
\sum_{j=1}^{i} \tilde{A}_{2 i \text { itj }}= & e_{1}+e_{4 i-4}+ \\
& \sum_{j=2}^{i-1}\left(e_{2 j-2}+e_{2 j-1}+e_{4 i-2-2 j}+e_{4 i \cdot 1-2 j}\right) \\
& +e_{2 i-2}+e_{2 i} 1 \\
= & \left.e_{1}+e_{4 i-4}+\sum_{\ell \in\{2, \ldots, 2 i} 3\right\} \quad e_{\ell}+\sum_{\ell \in\{2, \ldots, 2 i-3\}} e_{\ell} \\
& +\sum_{\ell \text { par }} e_{4 i-4-\ell}+\sum_{\ell \in\{1, \ldots, 2 i-4\}} e_{4 i-4-\ell}+e_{2 i-2}+e_{2 i-1} \\
= & e_{1}+e_{4 i-4}+\sum_{\ell=2}^{2 i-3} e_{\ell}+\sum_{\ell=2 i}^{4 i-5} e_{\ell}+e_{2 i-2}+e_{2 i-1} \\
= & \sum_{\ell=1}^{4 i-4} e_{\ell}=1_{4 i-4} \text { par }
\end{aligned}
$$

Isso implica que cada coluna de $\tilde{A}$ contém exatamente uma entrada não nula, ou seja, 1, no segundo conjunto de linhas. 
Os dois itens anteriores concluem a demonstraçāo.

Multiplicando por -1 as equaçōes ímpares em (3.11), este sistema é transformado no sistema equivalente (3.12) abaixo:

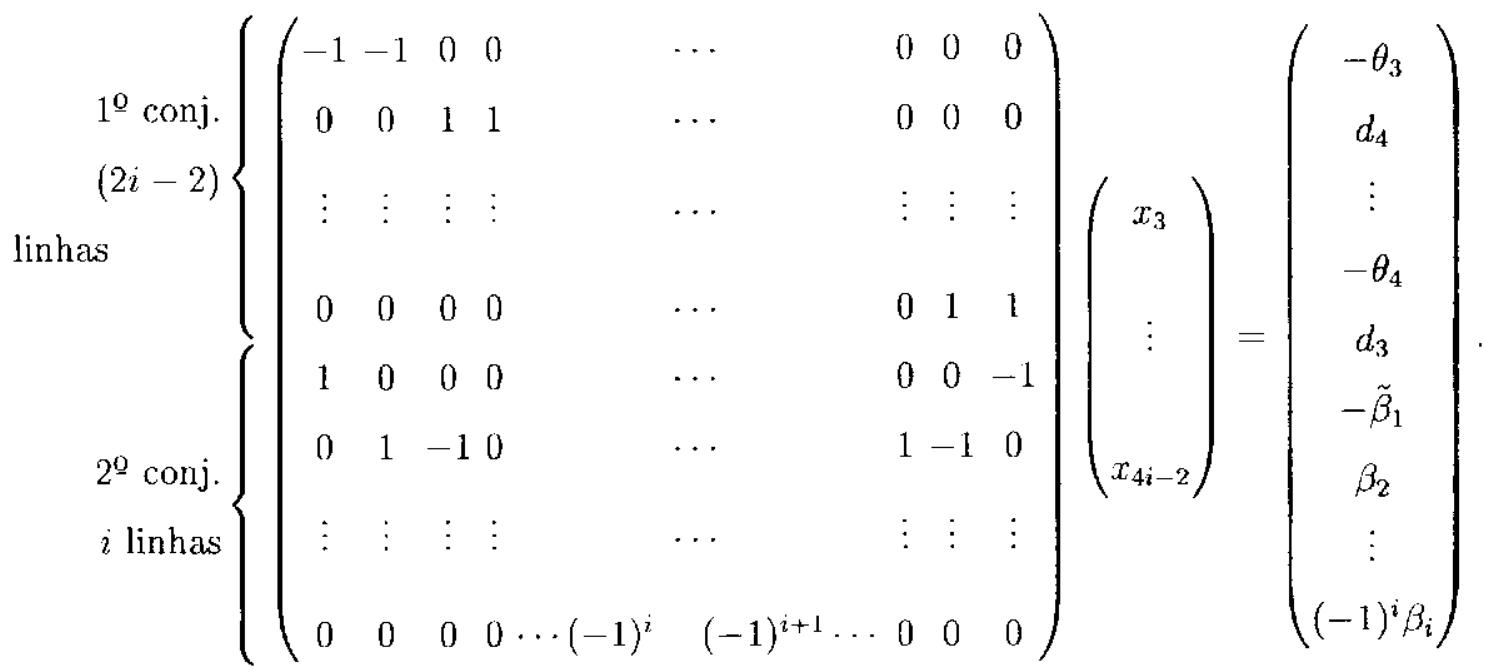

A matriz de coeficientes $A$ de (3.12) e seu vetor do lado direito $b$ herdam a partição estabclecida para os anteriores no sistema (3.9) e (3.11). As novas fómulas para a $j$-ésima linha e o elemento do lado direito no primeiro conjunto são:

$$
A_{j}-(-1)^{j}\left(\boldsymbol{e}_{2 j},-\boldsymbol{e}_{2 j}\right), \text { se } 1 \leq j \leq 2 i-2
$$

e

$$
b_{j}=\left\{\begin{array}{ll}
-\theta_{j+2}, & \text { se } 1 \leq j \leq i-1, j \text { impar } \\
d_{j+2}, & \text { se } 1 \leq j \leq i-1, j \text { par } \\
-\theta_{2 i+1-j}, & \text { se } i-1<j \leq 2 i \cdots 2, j \text { ímpar } \\
d_{2 i+1-j}, & \text { se } i-1<j<2 i-2, j \text { par }
\end{array},\right.
$$

e no segundo conjunto temos:

$$
\begin{aligned}
& A_{2 i-2+j}= \begin{cases}-\left(-e_{1}+e_{4 i}\right), & \text { se } j=1 \\
(-1)^{j}\left(e_{2 j-2}-e_{2 j-1}+e_{4 i-2-2 j}-e_{4 i-1-2 j}\right), & \text { se } 2 \leq j \leq i-1 \\
(-1)^{i}\left(e_{2 i}-e_{2 i}\right), & \text { se } j=i\end{cases} \\
& b_{2 i-2+j}= \begin{cases}-\tilde{\beta}_{1}, \quad \text { se } j-1 \\
(-1)^{j} \beta_{j}, \text { se } 2 \leq j \leq i\end{cases}
\end{aligned}
$$


Teorema 3.2.2 Cada coluna de A contém precisamente duas entradas não nulas: $1 e$ -1 .

Demonstraçāo: O Lema 3.2 .1 implica que $A$ tem duas entradas não nulas de valor absoluto 1 por coluna. Para concluir que este par é $1,-1$, é suficiente mostrar que somando todas as linhas produzimos o vetor nulo. Calculamos separadamente as somas das linhas no primeiro e segundo conjunto:

$$
\sum_{j=1}^{2 i-2} A_{j}=\sum_{j=1}^{2 i-2}(-1)^{j}\left(\boldsymbol{e}_{2 j-1}+\boldsymbol{e}_{2 j}\right)
$$

$\mathrm{e}$

$$
\begin{aligned}
\sum_{j=1}^{i} A_{2 i 2+j}= & \sum_{j=1}^{i}(-1)^{j} \overline{\bar{A}}_{2 i-2+j} \\
= & -\left(\cdots \boldsymbol{e}_{1}+\boldsymbol{e}_{4 i-4}\right) \\
& +\sum_{j=2}^{i-1}(-1)^{j}\left(\boldsymbol{e}_{2(j-1)} \cdots \boldsymbol{e}_{2(j-1)+1}+\boldsymbol{e}_{4 i-4-2(j-1)}-\boldsymbol{e}_{4 i-4-2(j-1)+1}\right) \\
& +(-1)^{i}\left(\boldsymbol{e}_{2 i-2}-\boldsymbol{e}_{2 i-1}\right) \\
= & \sum_{\ell=1}^{i-2}(-1)^{\ell+1}\left(\boldsymbol{e}_{2 \ell}+\boldsymbol{e}_{4 i-4-2 \ell}\right)+(-1)^{i} \boldsymbol{e}_{2 i-2}-\boldsymbol{e}_{4 i-4} \\
& +e_{1}+(-1)^{i+1} \boldsymbol{e}_{2 i-1}+\sum_{j=2}^{i-1}(-1)^{j+1}\left(\boldsymbol{e}_{2 j-1}+\boldsymbol{e}_{4 i-4-2(j-1)+1}\right) \\
= & \sum_{\ell=1}^{i-2}(-1)^{\ell+1} \boldsymbol{e}_{2 \ell}+(-1)^{i} \boldsymbol{e}_{2 i-2}+\sum_{k=i}^{2 i-3}(-1)^{k+1} \boldsymbol{e}_{2 k}-\boldsymbol{e}_{4 i-4} \\
& +e_{1}+\sum_{j=2}^{i-1}(-1)^{j+1} \boldsymbol{e}_{2 j-1}+(-1)^{i+1} e_{2 i-1}+\sum_{\ell=i+1}^{2 i-2}(-1)^{\ell+1} \boldsymbol{e}_{2 \ell-1} \\
& \sum_{j=1}^{2 i-2}(-1)^{j+1} \boldsymbol{e}_{2 j}+\sum_{j=1}^{2 i-2}(-1)^{j+1} \boldsymbol{e}_{2 j}{ }_{1} .
\end{aligned}
$$

As equações (3.13) e (3.14) implicam que

$$
\begin{aligned}
\sum_{j=1}^{3 i-2} A_{j} & =\sum_{j=1}^{2 i-2} A_{j}+\sum_{j-1}^{i} A_{2 i-2+j} \\
& =\sum_{j=1}^{2 i-2}(-1)^{j}\left(e_{2 j-1}+e_{2 j}\right)+\sum_{j-1}^{2 i-2}(-1)^{j+1}\left(e_{2 j}+e_{2 j-1}\right) \\
& =0_{4 i-4},
\end{aligned}
$$


onde $0_{m}$ é o vetor nulo de $\mathbb{R}^{m}$. Logo a soma das linhas de $A$ é o vetor nulo de $\mathbb{R}^{4 i-4}$, o que implica que as duas entradas não nulas de cada coluna de $A$ são 1 e -1 .

O Teorema 3.2 .2 implica, ver $[21$, p. 274], que $A$ (e assim também $\overline{\bar{A}}$ ) é uma matriz totalmente unimodular. Além disso, $A$ tem a estrutura da matriz de incidència de um digrafo, o que pode ser usado ao explorar o conjunto solução do sistema linear.

\section{Soluções do sistema reduzido}

Segue do Teorema 3.2 .2 que $A$ é totalmente unimodular e pode ser interpretada como a matriz de incidência de um digrafo $\mathcal{G}=(\mathcal{N}, \mathcal{A})$, com conjunto de nós $\mathcal{N}$ e conjunto de $\operatorname{arcos}$ (direcionados) $\mathcal{A}$. Se as entradas não nulas 1 e -1 da coluna $j$ pertencem às linhas $k$ e $\ell$, respectivamente, então o arco correspondente sai do nó $k$ e cntra no nó $\ell . \Lambda$ variável $x_{j+2}$ associada à coluna $j$ é interpretada como o fluxo no arco $j$ (partindo do nó $k$ e entrando no nó $\ell$ ), logo a $i$-ésima equação pode ser lida como

fluxo que entra no nó $i$ - fluxo que sai do nó $i=b_{i}$,

representando uma equação de equilíbrio de fluxo. Um valor positivo (resp., negativo) para $b_{i}$ é interpretado como a demanda (resp., -suprimento) do nó $i$.

Procuramos um fluxo inteiro não negativo que satisfaça as equaçōes de equilíbrio. Como $A$ é totalmente unimodular e $b$ é inteiro, é bem conkecido--ver, por exemplo, [21, p. 266-267]-que a existência de um fluxo não negativo implica na existência de um fluxo inteiro não negativo.

A estrutura de $\mathcal{G}$ torna possivel traduzir a existència de fluxo não negativo em um conjunto de restriçōes envolvendo o vetor demanda/suprimento $b$. A seguinte condição necessária para a existência de soluções para (3.12) é uma simples conseqüência do Teorema 3.2.2, e tem um enunciado natural em termos do modelo de fluxo descrito: suprimento deve ser igual a demanda. Como a soma das linhas de $A$ é igual ao vetor nulo em 
$\mathbb{R}^{4 i-4}$, a soma dos elementos de $b$ também deve ser zero:

$$
\begin{aligned}
0 & =-\sum_{j=3}^{i+1} \theta_{j}+\sum_{j=3}^{i+1} d_{j}+\sum_{j=1}^{i} b_{2 i-2+j} \\
& =-\sum_{j=3}^{i+1} \theta_{j}+\sum_{j=3}^{i+1} d_{j}-\tilde{\beta}_{1}+\sum_{j=2}^{i}(-1)^{j} \beta_{j} \\
& =-\sum_{j=3}^{i+1} \theta_{j}+\sum_{j=3}^{i+1} d_{j}-\left(\beta_{1}+\theta_{1}+\theta_{2}-d_{1}-d_{2}\right)+\sum_{j=2}^{i}(-1)^{j} \beta_{j} \\
& =-\sum_{j=1}^{i+1} \theta_{j}+\sum_{j=1}^{i+1} d_{j}+\sum_{j=1}^{i}(-1)^{j} \beta_{j} .
\end{aligned}
$$

Teorema 3.2.3 $O$ conjunto $\mathcal{A}$ pode ser particionado $\mathrm{em} i-1$ subconjunlos, cada um correspondendo a um ciclo não direcionado de $\mathcal{G}$. O j-ésimo ciclo é dado pela seguinte seqüência de nós e arcos:

Se j é ímpar:

$$
\begin{aligned}
& \{j,(j, 2 i-2+j), 2 i-2+j,(2 i-2+j, 2 i-1-j), \\
& 2 i-1-j,(2 i-1+j, 2 i-1-j), 2 i-1+j,(j, 2 i-1+j), j\}
\end{aligned}
$$

Se j é par:

$$
\begin{aligned}
& \{2 i-1-j,(2 i-1-j, 2 i-2+j), 2 i-2+j,(2 i-2+j, j), \\
& j,(2 i-1+j, j), 2 i-1+j,(2 i-1-j), 2 i-1+j), 2 i-1-j\}
\end{aligned}
$$

Demonstraçāo: Como cada ciclo tem quatro arcos, é suficiente mostrar que o digrafo contém os ciclos descritos e que eles são arco-disjuntos, já que então a união destes ciclos terá $4(i-1)$ arcos, que é precisamente a cardinalidade de $\mathcal{A}$.

Denotando por $\mathcal{N}_{j}$ o conjunto de nós $\{j, 2 i-1-j, 2 i-2+j, 2 i-1+j\}$, as linhas na matriz aumentada $A \mid b$ associada com nós em $\mathcal{N}_{j}$ são:

$$
\left(\begin{array}{c|c}
A_{j} & b_{j} \\
A_{2 i-1-j} & b_{2 i-1-j} \\
A_{2 i-2+j} & b_{2 i-2+j} \\
A_{2 i-1+j} & b_{2 i-1+j}
\end{array}\right)
$$

onde os elementos $b_{j}, b_{2 i-1-j}, b_{2 i-2+j}$ e $b_{2 i-1+j}$ são dados pela Tabela 3.1. 


\begin{tabular}{|c|c|c|}
\cline { 2 - 3 } \multicolumn{1}{c|}{} & \multicolumn{2}{c|}{$1 \leq j \leq i-1$} \\
\cline { 2 - 3 } \multicolumn{1}{c|}{} & $j$ odd & $j$ par \\
\hline$b_{j}$ & $-\theta_{j+2}$ & $d_{j+2}$ \\
\hline$b_{2 i-1-j}$ & $d_{j+2}$ & $-\theta_{j+2}$ \\
\hline$b_{2 i-2+j}$ & $-\bar{\beta}_{1}, \quad$ se $j=1$ & $\beta_{j}$ \\
\hline$b_{2 i-1+j}$ & $-\beta_{j}, \quad$ se $j \geq 3$ & \\
\hline
\end{tabular}

Tabela 3.1: Demanda/suprimento constantes para nós em $\mathcal{N}_{j}$

Portanto, os elementos do lado direito associados com nós em $\mathcal{N}_{j}$, independentemente da paridarde de $j$, são

$$
-\theta_{j+2},(-1)^{j}\left\{\begin{array}{ll}
\tilde{\beta}_{1}, & \text { se } j=1 \\
\beta_{j}, & \text { se } 1<j \leq i-1
\end{array}\right\} d_{j+2},(-1)^{j+1} \beta_{j+1},
$$

e a interseção dos subgrafos $\mathcal{G}_{j}$ e $\mathcal{G}_{\ell}$ induzidos respectivamente por $\mathcal{N}_{j}$ e $\mathcal{N}_{\ell}$, para $1<$ $j, \ell \leq i-1$, é:

$$
\mathcal{N}_{j} \cap \mathcal{N}_{\ell}= \begin{cases}\{2 i-1+j\}, & \text { se } \ell=j+1 \\ \{2 i-2+j\}, & \text { se } \ell=j-1 \\ \emptyset, & \text { caso contrário }\end{cases}
$$

Isto é, a interseção contém no máximo um nó, o que significa que os subgrafos induzidos $\mathcal{G}_{j}$ e $\mathcal{G}_{\ell}$ são arc0-disjuntos. Resta mostrar que $\mathcal{G}_{j}$ consiste do ciclo $\mathcal{C}_{j}$ da Figura 3.11 abaixo.

Obs.: o número escrito dentro do nó é o elemento do lado direito da equaçāo associada.

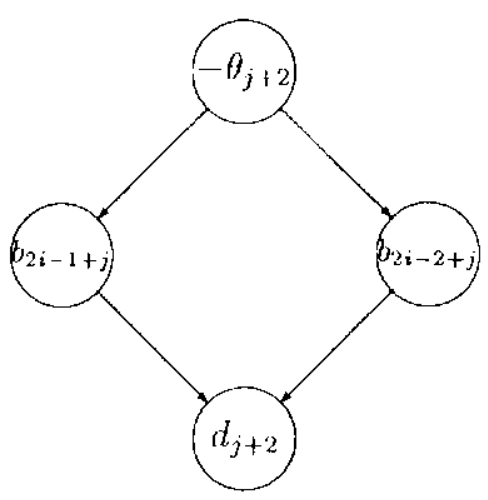

Figura 3.11: Ciclo $\mathcal{C}_{j}$. 
Para $j=1$ a submatriz em (3.16) se reduz a

$$
\left(\begin{array}{c|c}
A_{1} & -\theta_{3} \\
A_{2 i-2} & d_{3} \\
A_{2 i-1} & -\bar{\beta}_{1} \\
A_{2 i} & \beta_{2}
\end{array}\right)=\left(\begin{array}{c|c}
-e_{1}-e_{2} & -\theta_{3} \\
e_{4 i-5}+e_{4 i-4} & d_{3} \\
e_{1}-e_{4 i-4} & -\tilde{\beta}_{1} \\
e_{2}-e_{3}+e_{4 i-6}-e_{4 i-5} & \beta_{2}
\end{array}\right)
$$

Os arcos em $\mathcal{G}_{1}$ são aqueles que têm ambas as extremidades em $\mathcal{N}_{1}$. Assim a matriz de incidência de $\mathcal{G}_{1}$ contém as colunas de (3.17) que tem seus dois elementos não nulos na submatriz (3.17):

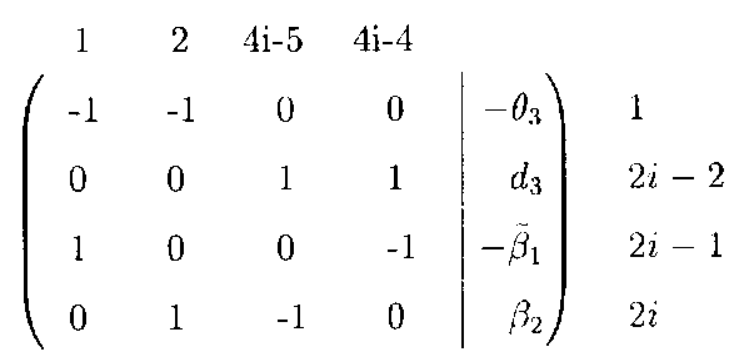

o que implica que $\mathcal{G}_{1}$ consiste dos ciclos não direcionados $\mathcal{C}_{\text {I }}$

$$
\{1,(1,2 i-1), 2 i-1,(2 i-1,2 i-2), 2 i-2,(2 i, 2 i-2), 2 i,(1,2 i), 1\}
$$

de acordo com o enunciado do teorema.

Se $2 \leq j<i-1$, a submatriz em (3.16) é, usando o fato que $2 i$ é par:

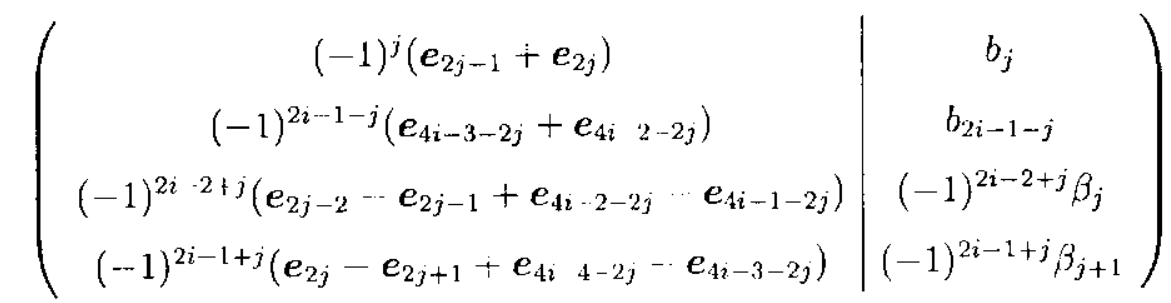

$$
\begin{aligned}
& \therefore(-1)^{j}\left(\begin{array}{c|c}
e_{2 j-1}+e_{2 j} & (-1)^{j} b_{j} \\
-e_{4 i-3-2 j}-e_{4 i-2} 2 j & (-1)^{j} b_{2 i-1-j} \\
e_{2 j \cdot 2}-e_{2 j-1}+e_{4 i-2 \cdot 2 j}-e_{4 i-1-2 j} & \beta_{j} \\
-e_{2 j}+e_{2 j+1}-e_{4 i-4-2 j}+e_{4 i-3-2 j} & -\beta_{j+1}
\end{array}\right)
\end{aligned}
$$


Isto implica que a matriz de incidência de $\mathcal{G}_{j}$ é:

$$
(-1)^{j}\left(\begin{array}{cccc|c}
2 \mathrm{j}-1 & 2 \mathrm{j} & 4 \mathrm{i}-3-2 \mathrm{j} & 4 \mathrm{i}-2-2 \mathrm{j} & \\
1 & 1 & 0 & 0 & (-1)^{j} b_{j} \\
0 & 0 & -1 & -1 & (-1)^{j} b_{2 i-1-j} \\
-1 & 0 & 0 & 1 & \beta_{j} \\
0 & -1 & 1 & 0 & -\beta_{j+1}
\end{array}\right) \begin{aligned}
& j \\
& 2 i-1-j \\
& 2 i-2+j \\
& 2 i-1+j
\end{aligned}
$$

Desta matriz de incidência e dos valores de $b_{j}$ e $b_{2 i-1-j}$ dados na Tabela 3.1 , concluímos que $\mathcal{G}_{j}$ é o ciclo $\mathcal{C}_{j}$ deserito na Figura 3.11 .

Finalmente, se $j=i-1$, a subrnatriz em (3.16) tem os valores

$$
\begin{gathered}
\left(\begin{array}{c|c}
(-1)^{i-1}\left(e_{2 i-3}+e_{2 i-1}\right) & b_{i-1} \\
(-1)^{i}\left(e_{2 i-1}+e_{2 i}\right) & b_{i} \\
(-1)^{3 i-3}\left(e_{2 i-4}-e_{2 i-3}+e_{2 i}-e_{2 i+1}\right) & (-1)^{3 i-3} \beta_{i-1} \\
(-1)^{3 i-2}\left(e_{2 i-2}-e_{2 i-1}\right) & (-1)^{2 i-1+j} \beta_{i}
\end{array}\right) \\
=(-1)^{i-1}\left(\begin{array}{c|c}
(-1)^{i-1} b_{i-1} \\
e_{2 i-3}+e_{2 i-2} \\
-e_{2 i-1}-e_{2 i} & (-1)^{i-1} b_{i} \\
e_{2 i}-e_{2 i-3}+e_{2 i}-e_{2 i+1} & \beta_{i-1} \\
-e_{2 i-2}+e_{2 i-1} & -\beta_{i}
\end{array}\right) .
\end{gathered}
$$

O subgrafo $\mathcal{G}_{i-1}$ tem, portanto, matriz de incidencia

$$
(-1)^{i-1}\left(\begin{array}{cccc|c}
2 \mathrm{i}-3 & 2 \mathrm{i}-2 & 2 \mathrm{i}-1 & 2 \mathrm{i} \\
1 & 1 & 0 & 0 & (-1)^{i-1} b_{i-1} \\
0 & 0 & -1 & -1 & (-1)^{i-1} b_{i} \\
-1 & 0 & 0 & 1 & \beta_{i-1} \\
0 & -1 & 1 & 0 & -\beta_{i}
\end{array}\right) \begin{aligned}
& i \\
& 3 i-3 \\
& 3 i-2
\end{aligned}
$$

Esta matriz e a Tabela 3.1 implicam que $\mathcal{G}_{i-1}$ consiste de $\mathcal{C}_{i-1}$, como desejado.

O Teorema 3.2.3 implica que o digrafo $\mathcal{G}$ tem a estrutura descrita na Figura 3.12. Isto é, o grafo obtido removendo a orientação dos arcos de $\mathcal{G}$ pode ser decomposto em $i-1$ componentes biconexas, onde cada uma delas é um ciclo com quatro nós e arcos. Nós com índices $2 i, 2 i+1, \ldots, 3 i-3$ são nós de corte, já que a remoção de qualquer deles desconecta o grafo. Note que estes nós correspondem a linhas no segundo conjunto da 
partiçāo. De fato cada linhas no segundo conjunto está associada a um nó de corte, exceto a primeira e a última.

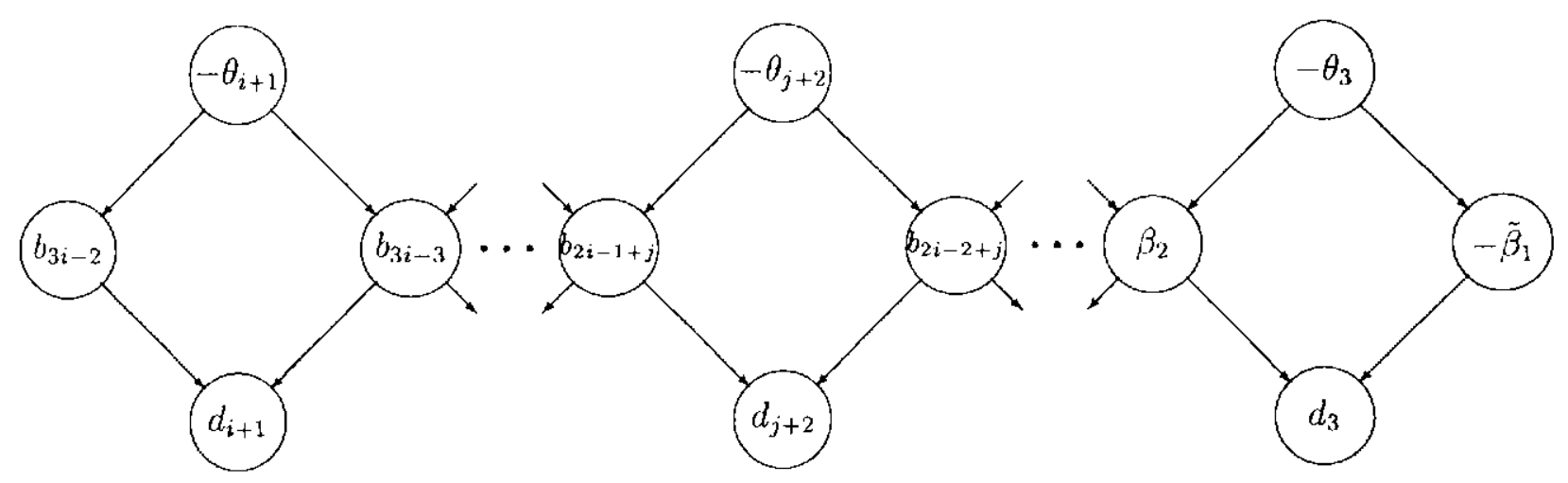

Figura 3.12: Estrutura de digrafo $\mathcal{G}$.

Teorema 3.2.4 Se a equação (3.15) se mantém, o sistema (3.12) é equivalente a um conjunto de $i-1$ sistemas lineares independentes. O j-ésimo sistema é dado por

$$
\left(\begin{array}{rrrr}
-1 & -1 & 0 & 0 \\
0 & 0 & 1 & 1 \\
1 & 0 & 0 & -1 \\
0 & 1 & -1 & 0
\end{array}\right)\left(\begin{array}{l}
w_{1}^{j} \\
w_{2}^{j} \\
w_{3}^{j} \\
w_{4}^{j}
\end{array}\right)=\left(\begin{array}{c}
-\theta_{j \mid 2} \\
d_{j+2} \\
\hat{\beta}_{j} \\
-\hat{\beta}_{j}+\theta_{j+2}-d_{j+2}
\end{array}\right),
$$

onde

$$
\begin{gathered}
\hat{\beta}_{j}=\sum_{\ell=1}^{j}(-1)^{\ell} \beta_{\ell}-\sum_{\ell=1}^{j+1}\left(\theta_{\ell}-d_{\ell}\right), \quad \text { para } j=1, \ldots, i-1, \\
w^{j}=\left\{\begin{array}{ll}
\left(x_{2 j+1}, x_{2 j+2}, x_{4 i-1-2 j}, x_{4 i-2 j}\right), & \text { se } 1 \leq j \leq i-1 \text { é ímpar } \\
\left(x_{4 i-2 j}, x_{4 i-1-2 j}, x_{2 j+2}, x_{2 j+1}\right), & \text { se } 1 \leq j \leq i-1 \text { é par }
\end{array} .\right.
\end{gathered}
$$

Demonstração: $A$ decomposição de um problema de fluxo em um grafo em um conjunto equivalente de problemas nas componentes biconexas do grafo é um resultado bem conhecido, como mencionado em [14]. Esta decomposiçāo envolve o conceito da árvore de corte do grafo ([22, p. 90-92]). É talvez mais fácil verificar esta equivalência diretamente por meio de uma simples prova de indução. 
Considere as equaçöes $1,2 i-2$ e $2 i-1$ de (3.12):

$$
\left(\begin{array}{rrrr}
-1 & -1 & 0 & 0 \\
0 & 0 & 1 & 1 \\
1 & 0 & 0 & -1
\end{array}\right)\left(\begin{array}{c}
x_{3} \\
x_{4} \\
x_{4 i} 3 \\
x_{4 i} \cdot 2
\end{array}\right)=\left(\begin{array}{c}
-\theta_{3} \\
d_{3} \\
-\tilde{\beta}_{1}
\end{array}\right)
$$

Somando estas equações à equação $2 i$ obtemos

$$
\begin{aligned}
-x_{5}+x_{4 i-4} & =\beta_{2}-\tilde{\beta}_{1}-\theta_{3}+d_{3} \\
& =\beta_{2}-\left(\beta_{1}+\theta_{1}+\theta_{2}-d_{1}-d_{2}\right)-\theta_{3}+d_{3} \\
& =\beta_{2}-\beta_{1}-\sum_{\ell=1}^{3}\left(\theta_{\ell}-d_{\ell}\right)=\hat{\beta}_{2}
\end{aligned}
$$

Trocando a equação $2 i$ de (3.12) com a equação acima conseguimos um sistema equivalente, já que somente operaçōes elementares de linha foram executadas. No novo sistema, as variáveis $x_{3}, x_{4}, x_{4 i-3}$ e $x_{4 i-2}$ aparecem apenas nas equações $1,2 i-2,2 i-1$ e estas equações não contêm nenhuma outra variável. Este novo sistema pode, portanto, ser decomposto no par de sistemas lineares independentes:

$$
\begin{aligned}
\left(\begin{array}{rrrr}
-1 & -1 & 0 & 0 \\
0 & 0 & 1 & 1 \\
1 & 0 & 0 & -1
\end{array}\right)\left(\begin{array}{c}
x_{3} \\
x_{4} \\
x_{4 i-3} \\
x_{4 i \cdot 2}
\end{array}\right) & =\left(\begin{array}{c}
-\theta_{3} \\
d_{3} \\
-\tilde{\beta}_{1}
\end{array}\right) \\
\left(\begin{array}{c}
\bar{A}_{2} \\
\vdots \\
\tilde{A}_{2 i-3} \\
\cdots \\
\tilde{A}_{2 i} \\
\vdots \\
\tilde{A}_{3 i-2}
\end{array}\right)\left(\begin{array}{c}
x_{5} \\
\vdots \\
x_{4 i-4}
\end{array}\right) & =\left(\begin{array}{c}
d_{4} \\
\vdots \\
-\theta_{4} \\
\cdots \\
\hat{\beta}_{2} \\
b_{2 i} \\
\vdots \\
b_{3 i-2}
\end{array}\right),
\end{aligned}
$$

onde a linha $\tilde{A}_{j}$ é obtida de $A_{j}$ eliminando quatro colunas: as duas primeiras e duas últimas. Como $A$ é a matriz de incidência do digrafo descrito na Figura 3.12 e as colunas 
e linhas eliminadas estāo associadas aos arcos e nós das componentes biconexas $\mathcal{C}_{1}$, com exceção do nó de corte, a matriz

$$
\tilde{A}=\left(\begin{array}{c}
\tilde{A}_{2} \\
\vdots \\
\tilde{A}_{2 i-3} \\
\tilde{A}_{2 i} \\
\vdots \\
\tilde{A}_{3 i-2}
\end{array}\right)
$$

é a matriz de incidência do digrafo dado pela união das componentes restantes: $\mathcal{C}_{2}, \ldots$, $\mathcal{C}_{i-1}$

Além disso, usando o fato que $\tilde{\beta}_{1}=\hat{\beta}_{1}$, temos que o sistema (3.23) abaixo é equivalente ao sistema (3.21), já que ele é obtido do segundo anexando uma equação redundante:

$$
\left(\begin{array}{rrrr}
-1 & -1 & 0 & 0 \\
0 & 0 & 1 & 1 \\
1 & 0 & 0 & -1 \\
0 & 1 & -1 & 0
\end{array}\right)\left(\begin{array}{c}
x_{3} \\
x_{4} \\
x_{4 i-3} \\
x_{4 i-2}
\end{array}\right)=\left(\begin{array}{c}
-\theta_{3} \\
d_{3} \\
\hat{\beta}_{1} \\
-\hat{\beta}_{1}+\theta_{3}-d_{3}
\end{array}\right) .
$$

Note que (3.23) é o sistema (3.18), para $i=1$, e o vetor de incógnitas é $w^{1}$.

Suponha por indução que, após $k$ passos análogos, um conjunto de $k+1$ sistemas, equivalente a (3.12), foi obtido, como segue: $k$ sistemas do tipo (3.18), para $j=1, \ldots$, $k$, e o sistema

$$
\left(\begin{array}{c}
\tilde{A}_{k+1} \\
\vdots \\
\tilde{\tilde{A}}_{2 i-2} k \\
\ldots \ldots \ldots \\
\tilde{\tilde{A}}_{2 i-1+k} \\
\vdots \\
\tilde{\bar{A}}_{3 i-2}
\end{array}\right)\left(\begin{array}{c}
x_{3+2 k} \\
\vdots \\
x_{4 i-2-2 k}
\end{array}\right)=\left(\begin{array}{c}
b_{k+1} \\
\vdots \\
b_{2 i-2} k \\
\ldots \ldots \ldots \\
\hat{\beta}_{k+1} \\
b_{2 i+k} \\
\vdots \\
b_{3 i-2}
\end{array}\right),
$$

cuja matriz de coeficientes $\tilde{\tilde{A}}$ é a matriz de incidência do digrafo dado por $\cup_{j=k+1}^{i-1} \mathcal{C}_{j}$, obtido eliminando de $A$ as colunas e linhas associadas com os arcos e nós em $\cup_{j=1}^{k} \mathcal{C}_{j}$, com exceçāo do nó de corte $\mathcal{C}_{k} \cap \mathcal{C}_{k+1}$. 
As equações associadas com os nós em $\mathcal{C}_{k+1}$, com exceção do nó de corte $\mathcal{C}_{k+1} \cap \mathcal{C}_{k+2}$, são

$$
(-1)^{k}\left(\begin{array}{rrrr}
-1 & -1 & 0 & 0 \\
0 & 0 & 1 & 1 \\
1 & 0 & 0 & -1
\end{array}\right)\left(\begin{array}{c}
x_{2 k+3} \\
x_{2 k+4} \\
x_{4 i-3 \cdot 2 k} \\
x_{4 i 2} 2 \cdot 2 k
\end{array}\right)=\left(\begin{array}{c}
b_{k+1} \\
b_{2 i} 2-k \\
\hat{\beta}_{k+1}
\end{array}\right)
$$

A equação associada com o nó $\mathcal{C}_{k+1} \cap \mathcal{C}_{k+2}$ é

$$
(-1)^{k}\left(x_{2 k+4}-x_{2 k+5}+x_{4 i-4-2 k}-x_{4 i-3-2 k}\right)=b_{2 i+k}=(-1)^{k+2} \beta_{k+2}
$$

Somando as equações (3.25) e a equação (3.26), e usando a Tabela 3.1 temos

$$
\begin{aligned}
(-1)^{k}\left(-x_{2 k+5}+x_{4 i-4-2 k}\right) & =(-1)^{k+2} \beta_{k+2}-\theta_{k+3}+d_{k+3}+\hat{\beta}_{k+1} \\
& =\sum_{j=1}^{k+1}(-1)^{j} \beta_{j}-\sum_{j=1}^{k+3}\left(\theta_{j}-d_{j}\right) \\
& =\hat{\beta}_{k+2}
\end{aligned}
$$

Logo (3.24) é equivalente ao sistema obtido substituindo a equação (3.26) pela equação (3.27). Mas esta substituiçāo produz um sistema que pode novamente ser dividido em dois: $u m$ do tipo (3.18) para $j=k+1$ (no vetor de incógnitas $w^{k+1}$ ) e o outro cuja matriz de coeficiente é a matriz de incidência do digrafo $\cup_{j=k \mid 2}^{i-1} \mathcal{C}_{j}$ e cujo vetor do lado direito é dado por $b_{k+2}, \ldots, b_{2 i-3-k}, \hat{\beta}_{k+2}, b_{2 i+1+k}, \ldots, b_{3 i-2}$. Portanto, por indução, isto implica que (3.12) é equivalente a um conjunto de $(i-1)$ sistemas: sistemas $(i-2)$ são do tipo (3.18), para $j=1, \ldots, i-2$, e o $(i-1)$-ésimo sistema é

$$
(-1)^{i}\left(\begin{array}{rrrr}
-1 & -1 & 0 & 0 \\
0 & 0 & 1 & 1 \\
1 & 0 & 0 & -1 \\
0 & 1 & -1 & 0
\end{array}\right)\left(\begin{array}{c}
x_{2 i-1} \\
x_{2 i} \\
x_{2 i+1} \\
x_{2 i+2}
\end{array}\right)=\left(\begin{array}{cc}
b_{i} & 1 \\
b_{i} \\
\hat{\beta}_{i-1} \\
(-1)^{2} \beta_{i}
\end{array}\right) .
$$

Usando Tabela 3.1, somando os três primeiros elementos do lado direito de (3.28) obtemos

$$
\begin{aligned}
b_{i-1}+b_{i}+\hat{\beta}_{i-1} & =-\theta_{i+1}+d_{i+1}+\sum_{j=1}^{i-1}(-1)^{j} \beta_{1}-\sum_{j=1}^{i}\left(\theta_{j}-d_{j}\right) \\
& =\sum_{j=1}^{i-1}(-1)^{j} \beta_{1}-\sum_{j=1}^{i+1}\left(\theta_{j}-d_{j}\right)
\end{aligned}
$$


Como assumimos a equação (3.15), isto é, $(-1)^{i} \beta_{i}=-\left(\sum_{j=1}^{i-1}(-1)^{j} \beta_{1}-\sum_{j=1}^{i+1}\left(\theta_{j}-d_{j}\right)\right)$, a Tabela 3.1 e a equação (3.15) implicam que (3.28) é também do tipo (3.18) para $j=i-1$.

O Teorema 3.2.4 implica que a solução do sistema original (3.9) é o produto cartesiano de 4-uplas do tipo $q_{j}=\left(x_{2 j-1}, x_{2 j}, x_{4 i+1-2 j}, x_{4 i+2-2 j}\right)$, para $j=1, \ldots, i$. Na Subseção 3.2.1 vimos que $q_{1}$ tem um único valor, determinado pelas equaçōes $1,2,2 i+2$ e $2 i+1$ de (3.9). A 4-upla $q_{j}$, para $j=2, \ldots, i$, é uma reordenação de um vetor $w$ que deve satisfazer um sistema linear do tipo

$$
\left(\begin{array}{rrrr}
-1 & -1 & 0 & 0 \\
0 & 0 & 1 & 1 \\
1 & 0 & 0 & -1 \\
0 & 1 & -1 & 0
\end{array}\right)\left(\begin{array}{l}
w_{1} \\
w_{2} \\
w_{3} \\
w_{4}
\end{array}\right)=\left(\begin{array}{c}
-t \\
\delta \\
\xi \\
-\xi+t-\delta
\end{array}\right) .
$$

Lema 3.2.5 A solução geral do sistema (3.29) é dada por

$$
\left(\begin{array}{l}
w_{1} \\
w_{2} \\
w_{3} \\
w_{4}
\end{array}\right)=\left(\begin{array}{c}
\xi \\
t-\xi \\
\delta \\
0
\end{array}\right)+\alpha\left(\begin{array}{c}
1 \\
-1 \\
-1 \\
1
\end{array}\right)
$$

onde $\alpha \in \mathbb{R}$

Demonstraçāo: Substituindo, pode ser facilmente verificado que $(\xi, t-\xi, \delta, 0)$ é uma soluçāo de $(3.29)$ e que $(1,-1,-1,1)$ é uma solução da versão homogênea de (3.29). Como o espaço nulo da matriz de coeficientes de (3.29) tem dimensāo 1 e a diferença entre duas soluçōes é um vetor deste espaço nulo, a fórmula (3.30) descreve todas as soluções de (3.29).

Lema 3.2.6 Se $t, \xi$ e $\delta$ são inteiros, e $t$, $\delta$ são não negativos, o sistema (3.29) tem uma solução inteira não negativa se, e somente se, as seguintes desigualdades são válidas

$$
\left\{\begin{array}{l}
\delta \geq-\xi \\
t \geq \xi
\end{array}\right.
$$


Além disso, o número de soluções inteiras não negativas de (3.29) é igual ao número de inteiros no intervalo

$$
[\max \{0,-\xi\}, \min \{t-\xi, \delta\}]
$$

Demonstração: A matriz de coeficientes de (3.29) é uma submatriz de $A$ e assim é totalmente unimodular. Seu lado direito é inteiro. Estes fatos implicam que o sistema (3.29) tem solução inteira não negativa se, e somente se, ela tem uma soluçāo não negativa. O Lema 3.2.5 implica que (3.29) tem uma solução não negativa se, e somente se, as desigualdades (3.32) sāo satisfeitas:

$$
\begin{aligned}
\xi+\alpha & \geq 0 \\
t-\xi-\alpha & \geq 0 \\
\delta-\alpha & \geq 0 \\
\alpha & \geq 0
\end{aligned}
$$

Aplicando o método de Fourier-Motzkin (ver [1, p. 57-62]) para eliminar a variável $\alpha$ em (3.32), as seguintes desigualdades são obtidas:

$$
\begin{aligned}
t-\xi \geq & \alpha \\
\delta \geq & \alpha \\
& \alpha \geq-\xi \\
& \alpha \geq 0
\end{aligned}
$$

O Lema 3.2 .5 estabelece que a solução geral para (3.29) é dada por $(\xi, t-\xi, \delta, 0)+$ $\alpha(1,-1,-1,1)$. Portanto, para cada valor inteiro $\alpha$ no intervalo $[\max \{0,-\xi\}, \min \{t-\xi, \delta\}]$ existe uma solução inteira não negativa de (3.29).

Finalmente, o intervalo $[\max \{0,-\xi\}, \min \{t-\xi, \delta\}]$ é não vazio se, e somente se, as desigualdades abaixo são satisfeitas:

$$
\begin{aligned}
t-\xi & \geq 0 \\
\delta & \geq 0 \\
t-\xi & \geq-\xi \\
\delta & \geq-\xi
\end{aligned}
$$


As desigualdades (3.34) e (3.35) são satisfeitas, já que $\delta$ e $t$ sāo assumidos não negativos. As duas restantes constituem o sistema (3.31).

Reunimos abaixo as condições necessárias e suficientes para a existência de soluções inteiras nāo negativas de (3.9)

$$
\left\{\begin{aligned}
d_{2} & \geq \theta_{1} \\
\theta_{2} & \geq d_{1} \\
\sum_{j=1}^{i}(-1)^{j} \beta_{j}-\sum_{j=1}^{i+1}\left(\theta_{j}-d_{j}\right) & =0 \\
\theta_{j+2} & \geq \sum_{\ell=1}^{j}(-1)^{\ell} \beta_{\ell}-\sum_{\ell=1}^{j+1}\left(\theta_{\ell}-d_{\ell}\right), \quad \text { para } j=1, \ldots, i-1 \\
d_{j+2} & \geq-\left(\sum_{\ell=1}^{j}(-1)^{\ell} \beta_{\ell}-\sum_{\ell=1}^{j+1}\left(\theta_{\ell}-d_{\ell}\right)\right), \text { para } j=1, \ldots, i-1
\end{aligned}\right.
$$

O número de soluções é dado pelo produto $\prod_{j=1}^{i-1} n_{j}$, onde $n_{j}$ é o número de inteiros no intervalo $\left\{\max \left\{0,-\hat{\beta}_{j}\right\}, \min \left\{\theta_{j+2}-\hat{\beta}_{j}, d_{j+2}\right\}\right]$ e $\hat{\beta}_{j}=\sum_{\ell=1}^{j}(-1)^{\ell} \beta_{\ell}-\sum_{\ell=1}^{j+1}\left(\theta_{\ell}-d_{\ell}\right)$.

\subsubsection{Caso $\mathrm{n}=0 \mathrm{mod} 4$}

\section{O sistema linear}

Seja $4 \leq n=2 i$ um inteiro múltiplo de 4 (ou, equivalentemente, $2 \leq i$ é par). Queremos encontrar uma solução inteira não negativa do sistema linear: 


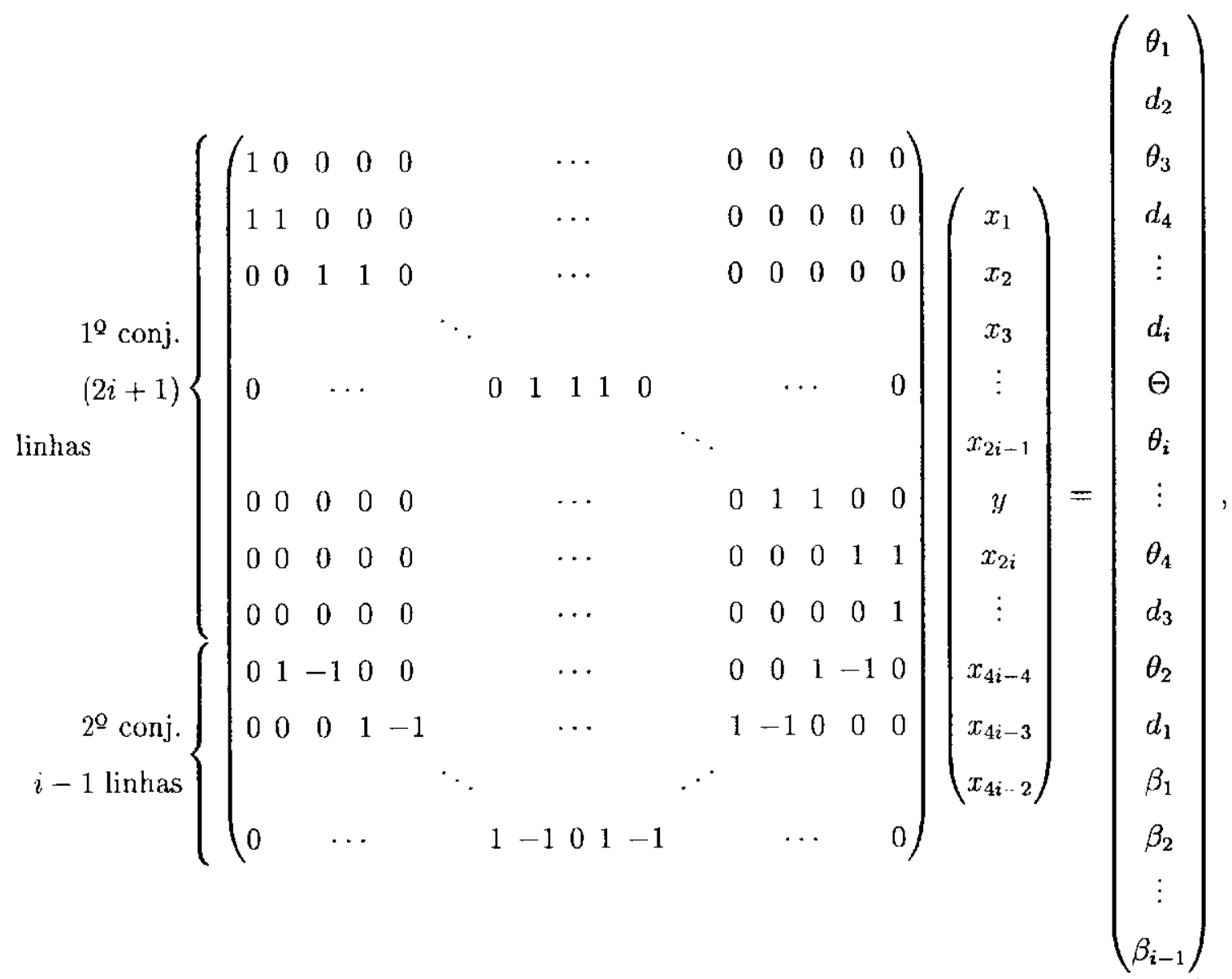

onde $\Theta, \theta_{j}$ e $d_{j}$, para $j=1, \ldots, i$, são inteiros positivos e $\beta_{j}$, para $j=1, \ldots, i-1$, são inteiros. O sistema tem $3 i$ equações e $4 i-1$ incógnitas, matriz de coeficientes $\bar{A}$ e vetor do lado dircito $\bar{b}$. As equações, linhas de $\bar{A}$ e $\bar{b}$ são particionados em dois conjuntos, contendo o primeiro $2 i+1$ equações e o seguinte $i-1$. As fórmulas para a $j$-ésima linha do primeiro grupo e o elemento correspondente do lado direito são

$$
\bar{A}_{j}= \begin{cases}\boldsymbol{e}_{1}, & \text { se } j=1 \\ \boldsymbol{e}_{2 j-3}+\boldsymbol{e}_{2 j-2}, & \text { se } 2 \leq j \leq i \\ \boldsymbol{e}_{2 i-1}+\boldsymbol{e}_{2 i}+\boldsymbol{e}_{2 i+1}, & \text { se } j=i+1 \\ \boldsymbol{e}_{2 j-2}+\boldsymbol{e}_{2 j-1}, & \text { se } i+2 \leq j \leq 2 i \\ \boldsymbol{e}_{4 i-1}, & \text { se } j=2 i+1\end{cases}
$$


e

$$
\bar{b}_{j}=\left\{\begin{array}{ll}
\theta_{j}, & \text { se } 1 \leq j \leq i, j \text { impar } \\
d_{j}, & \text { se } 1 \leq j \leq i, j \text { par } \\
\Theta, & \text { se } j=i+1 \\
\theta_{2 i+2 \cdots j}, & \text { se } i+2 \leq j \leq 2 i+1, j \text { par } \\
d_{2 i+2 \cdots j}, & \text { se } i+2 \leq j \leq 2 i+1, j \text { impar }
\end{array},\right.
$$

onde $\boldsymbol{e}_{j}$ é o $j$-ćsimo vetor da base canônica de $\mathbb{R}^{4 i-1}$. No segundo grupo, a $j$-ésima linha e respectivo elemento do lado dircito são como segue

$$
\bar{A}_{2 i+1+j}=e_{2 j}-e_{2 j+1}+e_{4 i-1-2 j}-e_{4 i-2 j}, \text { se } 1 \leq j<i-1
$$

e

$$
\bar{b}_{2 i+1+j}=\beta_{j}, \text { se } 1 \leq j \leq i-1
$$

Como no caso de $n$ ímpar, quatro variáveis em (3.38) sāo unicamente determinadas. O restante das variáveis devem satisfazer um sistema reduzido. A matriz de coeficiente do sistema reduzido nāo ć totalmente unimodular. No entanto, mostra-se na Subseçāo 3.2.2 que, se removemos a coluna associada à variável $x_{2 i}$, a matriz restante é totalmente unimodular. Exploramos este fato na Subseção 3.2.2 para produzir um conjunto de condições que é equivalente à existência de solução inteira não negativa do sistema reduzido.

As equações que podem ser usadas para eliminar as quatro variáveis em (3.38) são:

1. A primeira equação implica que $x_{1}=\theta_{1}$.

2. A primeira e a segunda equação implicam que $x_{2}=d_{2}-\theta_{1}$.

3. A $(2 i+1)$-ésima equação implica que $x_{4 i-2}=d_{1}$.

4. A $(2 i+1)$-ésima e $2 i$-ésima equações implicam que $x_{4 i-3}=\theta_{2}-d_{1}$.

Assim as desigualdades (3.10), já obtidas na Subseção 3.2.1, são condições necessárias e suficientes para a solução parcial estabelecida em 1-4 acima ser um vetor inteiro não negativo.

O sistema reduzido obtido com a remoção da 4-upla $\left(x_{1}, x_{2}, x_{4 i-3}\right)$ e das equações mencionadas em 1-4 acima é estudado a seguir. 


\section{O sistema reduzido}

Chegamos no sistema reduzido (3.39) abaixo após eliminar as equações $1,2,2 i$ e $2 i+1$ e as variáveis $x_{1}, x_{2}, x_{4 i-3}$ e $x_{4 i-2}$ em (3.38):

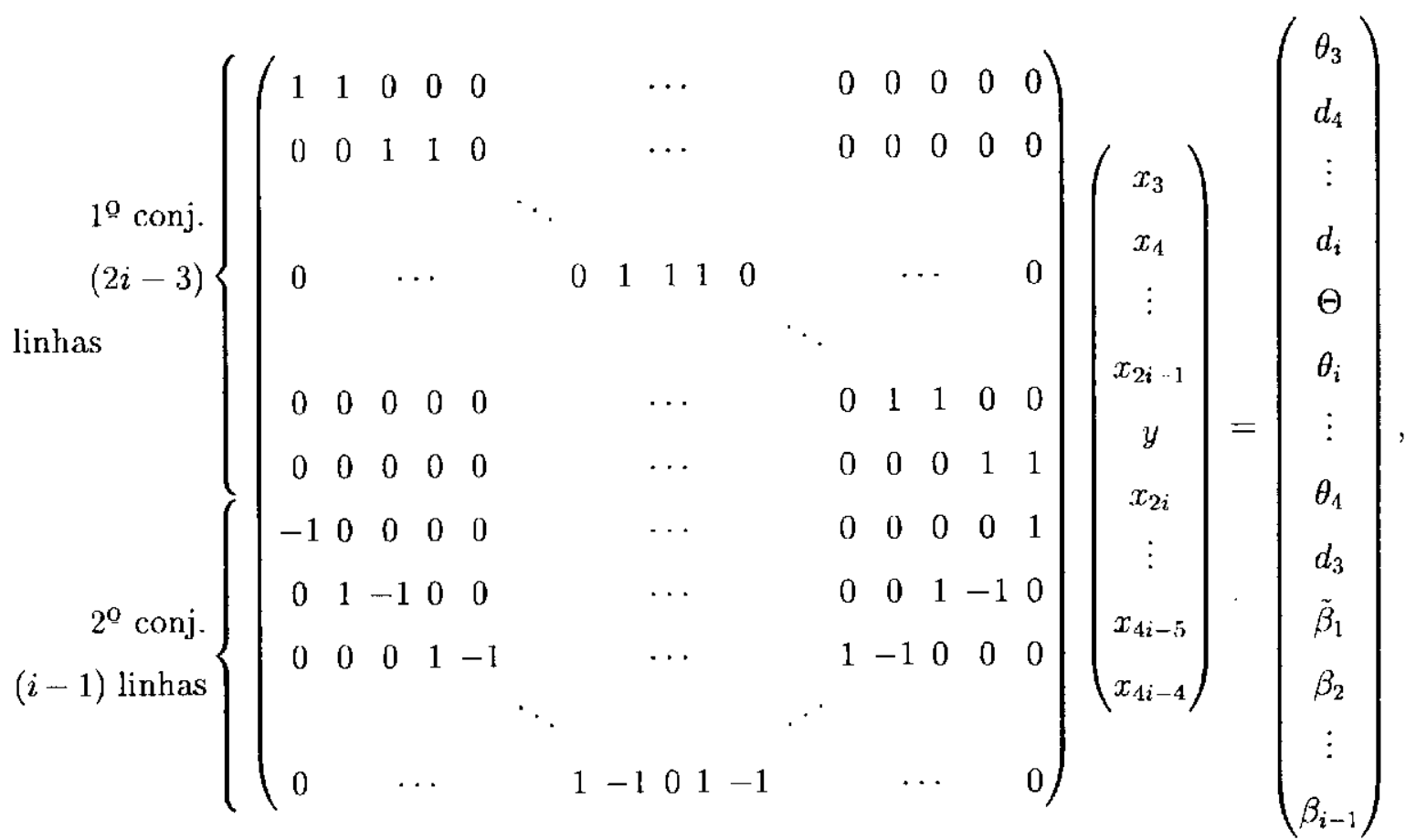

onde $\tilde{\beta}_{1}=\beta_{1}-x_{2}+x_{4 i-3}=\beta_{1}+\theta_{1}+\theta_{2}-d_{1}-d_{2}$. Este sistema reduzido tem $3 i-4$ equaçōes e $4 i-5$ incógnitas.

As linhas de $\overline{\bar{A}} \mathrm{e} \overline{\bar{b}}$, a matriz de coeficientes e o vetor do lado direito de (3.39), herdam a partição estabelecida para suas contrapartes em (3.38). Logo os dois conjuntos tem $2 i-3$ e $i-1$ linhas/elementos, respectivamente. As fórmulas para a $j$-ésima linha e elemento do lado direito devem ser atualizadas a fim de considerar eliminação de linhas e colunas (cquações e variáveis). No primeiro grupo temos:

$$
\overline{A_{j}}= \begin{cases}\boldsymbol{e}_{2 j-1}+\boldsymbol{e}_{2 j}, & \text { se } 1<j<i-2 \\ \boldsymbol{e}_{2 i-3}+\boldsymbol{e}_{2 i-2}+\boldsymbol{e}_{2 i-1}, & \text { se } j=i-1 \\ \boldsymbol{e}_{2 j}+\boldsymbol{e}_{2 j+1}, & \text { se } i \leq j \leq 2 i-3\end{cases}
$$


$\mathrm{e}$

$$
\overline{\bar{b}}_{j}=\left\{\begin{array}{ll}
\theta_{j+2}, & \text { se } 1 \leq j \leq i-2, j \text { impar } \\
d_{j+2}, & \text { se } 1 \leq j \leq i-2, j \text { par } \\
\Theta, & \text { se } j=i-1 \\
\theta_{2 i-j}, & \text { se } i \leq j \leq 2 i-3, j \text { par } \\
d_{2 i-j}, & \text { se } i \leq j \leq 2 i-3, j \text { impar }
\end{array} .\right.
$$

No segundo conjunto, as fómulas são:

$$
\overline{\bar{A}}_{2 i-3+j}= \begin{cases}-e_{1}+e_{4 i-5}, & \text { sc } j=1 \\ e_{2 j-2}-e_{2 j-1}+e_{4 i-3-2 j}-e_{4 i-2-2 j}, & \text { se } 2 \leq j \leq i-1\end{cases}
$$

e

$$
\overline{\bar{b}}_{2 i-3+j}=\left\{\begin{array}{l}
\tilde{\beta}_{1}, \quad \text { se } j=1 \\
\beta_{j}, \quad \text { se } 2 \leq j \leq i-1
\end{array} .\right.
$$

Note que $\boldsymbol{e}_{j}$ é agora o $j$-ésimo vetor da base canônica de $\mathbb{R}^{4 i-5}$.

Lema 3.2.7 Cada coluna de $\overline{\bar{A}}$, exceto a coluna (central) $2 i-2$, contém exatamente dois coeficientes não nulos, um no primeiro conjunto de linhas e o outro no segundo conjunto. A coluna $2 i-2$ contém somente um elemento não nulo, 1 , na linha $i-1$.

Demonstração: Considere a matriz $\tilde{A}$ com cada entrada de $\overline{\bar{A}}$ substituída por seu valor absoluto. Somando as linhas no primeiro conjunto:

$$
\begin{aligned}
\sum_{j=1}^{2 i-3} \tilde{A}_{j}= & \sum_{j=1}^{i-2}\left(\boldsymbol{e}_{2 j-1}+\boldsymbol{e}_{2 j}\right)+\boldsymbol{e}_{2 i-3}+\boldsymbol{e}_{2 i-2}+\boldsymbol{e}_{2 i-1}+\sum_{j=i}^{2 i-3}\left(\boldsymbol{e}_{2 j}+\boldsymbol{e}_{2 j+1}\right) \\
= & \sum_{\substack{j \in\{1, \ldots, 2 i-4\} \\
j \text { impar }}} \boldsymbol{e}_{j}+\sum_{\substack{j \in\{1, \ldots, 2 i-4\} \\
j \text { par }}} \boldsymbol{e}_{j}+\boldsymbol{e}_{2 i-3}+\boldsymbol{e}_{2 i-2}+\boldsymbol{e}_{2 i-1}+ \\
& \sum_{\substack{j \in\{2 i, \ldots, 4 i-5\} \\
j \text { par }}} \boldsymbol{e}_{j}+\sum_{j \in\{2 i, \ldots, 4 i-5\}} \boldsymbol{e}_{j} \\
= & \mathbf{1}_{4 i-5 .} .
\end{aligned}
$$


Somando as linhas no segundo conjunto:

$$
\begin{aligned}
& \sum_{j=1}^{i \cdot 1} \tilde{A}_{2 i-3+j}=e_{1}+e_{4 i-5}+\sum_{j=2}^{i-1}\left(e_{2 j-2}+e_{2 j-1}+e_{4 i} 32 j+e_{4 i-2-2 j}\right) \\
& =e_{1}+\sum e_{j}+\sum e_{j}+ \\
& j \in\{2, \ldots, 2 i-3\} \quad j \in\{2, \ldots, 2 i-3\} \\
& j \text { par } \quad j \text { impar } \\
& \sum \boldsymbol{e}_{j}+\sum e_{j}+\boldsymbol{e}_{4 i-5} \\
& j \in\{2 i-1, \ldots, 4 i-6\} \quad j \in\{2 i-1, \ldots, 4 i 6\} \\
& j \text { impar } j \text { par } \\
& =1_{4 i .5}-e_{2 i \cdot 2} \text {. }
\end{aligned}
$$

A primeira soma implica que cada coluna contém um elemento não nulo, 1 (já que as linhas no primciro conjunto tem entradas não negativas), no primeiro conjunto de linhas. Incidentemente, a linha $i-1$ contribui com a entrada não nula na coluna $2 i-2$. A segunda soma implica que cada coluna, com exceção da coluna $2 i-2$ contém um elemento não nulo, com valor absoluto 1 , no segundo conjunto de linhas. A coluna $2 i-2$ não contém elementos não nulos no segundo conjunto. Assim o lema está demonstrado.

Multiplicando por -1 a $j$-ésima equação, onde escolhemos $j$ ímpar se $1 \leq j \leq i-1$, e par $j$ se $i \leq j \leq 3 i-4$. O sistema resultante é

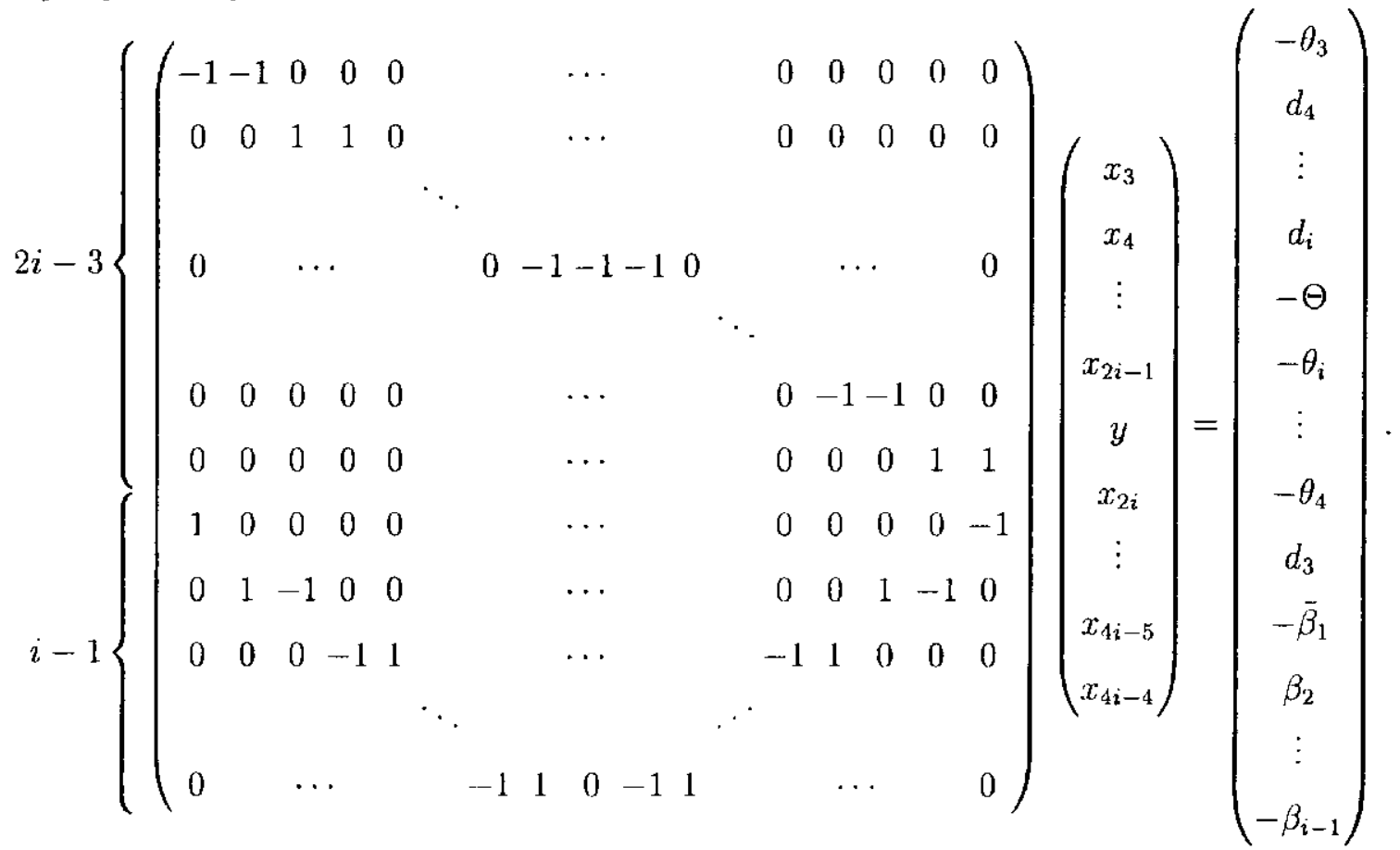


O novo sistema (3.40) tem matriz de coeficiente $\hat{A}$ e o vetor do lado direito $\hat{b}$. Como antes ele estão particionados em dois conjuntos. A j-ésima linha e o elemento do lado direito no primeiro conjunto são dados por:

$$
\begin{aligned}
& \hat{A}_{j}= \begin{cases}(-1)^{j}\left(e_{2 j-1}+e_{2 j}\right), & \text { se } 1 \leq j \leq i-2 \\
-e_{2 i-3}-e_{2 i-2}-e_{2 i-1}, & \text { se } j=i-1 \\
(-1)^{j+1}\left(e_{2 j}+e_{2 j+1}\right), & \text { se } i \leq j \leq 2 i-3\end{cases} \\
& \hat{b}_{j}= \begin{cases}-\theta_{j+2}, & \text { se } 1 \leq j \leq i-2, j \text { impar } \\
d_{j+2}, & \text { se } 1 \leq j \leq i-2, j \text { par } \\
-\Theta, & \text { se } j=i-1 \\
-\theta_{2 i-j}, & \text { se } i \leq j \leq 2 i-3, j \text { par } \\
d_{2 i-j}, & \text { se } i<j \leq 2 i-3, j \text { ímpar }\end{cases}
\end{aligned}
$$

No segundo conjunto, as fórmulas sāo:

$$
\begin{aligned}
& \hat{A}_{2 i-3+j}= \begin{cases}\boldsymbol{e}_{1}-\boldsymbol{e}_{4 i-5}, & \text { se } j=1 \\
(-1)^{j}\left(\boldsymbol{e}_{2 j-2}-\boldsymbol{e}_{2 j-1}+\boldsymbol{e}_{4 i-3-2 j}-\boldsymbol{e}_{4 i-2-2 j}\right), & \text { se } 2 \leq j \leq i-1\end{cases} \\
& \hat{b}_{2 i-3+j}=\left\{\begin{array}{ll}
-\tilde{\beta}_{1}, & \text { se } j=1 \\
(-1)^{j} \beta_{j}, & \text { se } 2 \leq j \leq i-1
\end{array} .\right.
\end{aligned}
$$

Teorema 3.2.8 A j-ésima coluna de $\hat{A}$ contém

$$
\begin{aligned}
& \text { duas entradas não nulas, } 1 \text { e }-1, \quad \text { se } j \neq 2 i-2,2 i-1 \text {, } \\
& \text { uma entrada não nula, }-1, \quad \text { se } j=2 i-2 \text {, } \\
& \text { duas entradas näo nulas, ambas }-1 \text {, se } j=2 i-1 \text {. }
\end{aligned}
$$

Demonstração: Pelo Lema 3.2 .7 e o fato que as entradas de $\hat{A}$ pertencem a $\{0,-1,1\}$, é suficiente mostrar que a soma das linhas de $\hat{A}$ resultam em $-e_{2 i-2}-2 e_{2 i-1}$.

Somando as linhas no primeiro conjunto, temos:

$$
\begin{aligned}
\sum_{j=1}^{2 i-3} \hat{A}_{j}= & \sum_{j=1}^{i-2}(-1)^{j}\left(\boldsymbol{e}_{2 j-1}+\boldsymbol{e}_{2 j}\right)-\boldsymbol{e}_{2 i-3}-\boldsymbol{e}_{2 i-2}-\boldsymbol{e}_{2 i-1}+\sum_{j=i}^{2 i-3}(-1)^{j+1}\left(\boldsymbol{e}_{2 j}+\boldsymbol{e}_{2 j+1}\right) \\
= & \sum_{j=1}^{i-1}(-1)^{j} \boldsymbol{e}_{2 j-1}+\sum_{j=1}^{i-2}(-1)^{j} \boldsymbol{e}_{2 j}-\boldsymbol{e}_{2 i-2}-\boldsymbol{e}_{2 i-1}+ \\
& \sum_{j=i}^{2 i-3}(-1)^{j+1} \boldsymbol{e}_{2 j}+\sum_{j=i+1}^{2 i-2}(-1)^{j} \boldsymbol{e}_{2 j-1} .
\end{aligned}
$$


Para o segundo conjunto, obtemos:

$$
\begin{aligned}
\sum_{j=1}^{i-1} \hat{A}_{2 i-3+j}= & \boldsymbol{e}_{1}-\boldsymbol{e}_{4 i-5}+\sum_{j=2}^{i-1}(-1)^{j}\left(\boldsymbol{e}_{2 j-2}-\boldsymbol{e}_{2 j-1}+\boldsymbol{e}_{4 i-3-2 j}-\boldsymbol{e}_{4 i-2-2 j}\right) \\
= & \boldsymbol{e}_{1}-\boldsymbol{e}_{4 i-5}+\sum_{j=2}^{i-1}(-1)^{j} \boldsymbol{e}_{2 j-2}+\sum_{j=2}^{i-1}(-1)^{j+1} \boldsymbol{e}_{2 j-1}+ \\
& \sum_{j=2}^{i-1}(-1)^{j} \boldsymbol{e}_{4 i-3-2 j}+\sum_{j-2}^{i-1}(-1)^{j+1} \boldsymbol{e}_{4 i-2-2 j} \\
= & \sum_{j=1}^{i-2}(-1)^{j+1} \boldsymbol{e}_{2 j}+\boldsymbol{e}_{1}+\sum_{j=2}^{i-1}(-1)^{j+1} \boldsymbol{e}_{2 j-1}+ \\
& \sum_{j=i}^{2 i 3}(-1)^{j+1} \boldsymbol{e}_{2 j-1}-\boldsymbol{e}_{4 i-5}+\sum_{j=i}^{2 i-3}(-1)^{j} \boldsymbol{e}_{2 j} \\
= & \sum_{j=1}^{i-2}(-1)^{j+1} e_{2 j}+\sum_{j=1}^{i-1}(-1)^{j+1} e_{2 j-1}+ \\
& \sum_{j=i}^{2 i-2}(-1)^{j+1} \boldsymbol{e}_{2 j-1}+\sum_{j=i}^{2 i-3}(-1)^{j} \boldsymbol{e}_{2 j} .
\end{aligned}
$$

As equaçōes (3.41) e (3.42) implicam no resultado descjado.

O Teorema 3.2.8 implica que a matriz obtida de $\hat{A}$ removendo a coluna $2 i-1$ é totalmente unimodular. Para explorar este fato, consideramos o sistema linear parametrizado obtido interpretando-se $x_{2 i}$ (a variável associada a coluna $2 i-1$ ) como um parâmetro. Assim, para cada valor fixado $p$ deste parâmetro temos um sistema linear com lado direito igual a $\hat{b}$ menos $p$ vezes a coluna $2 i-1$ de $\hat{A}$. Além disso, se adicionamos a equação redundante $e_{2 i-2} x=-\sum_{j=1}^{3 i-4} \hat{b}_{j}-2 p$, a matriz de coeficiente do sistema parametrizado será a matriz de incidência de um digrafo. 


\section{Soluções do sistema parametrizado}

O sistema parametrizado, correspondendo a $x_{2 i}=p$, descrito na subseção anterior tem a seguinte estrutura:

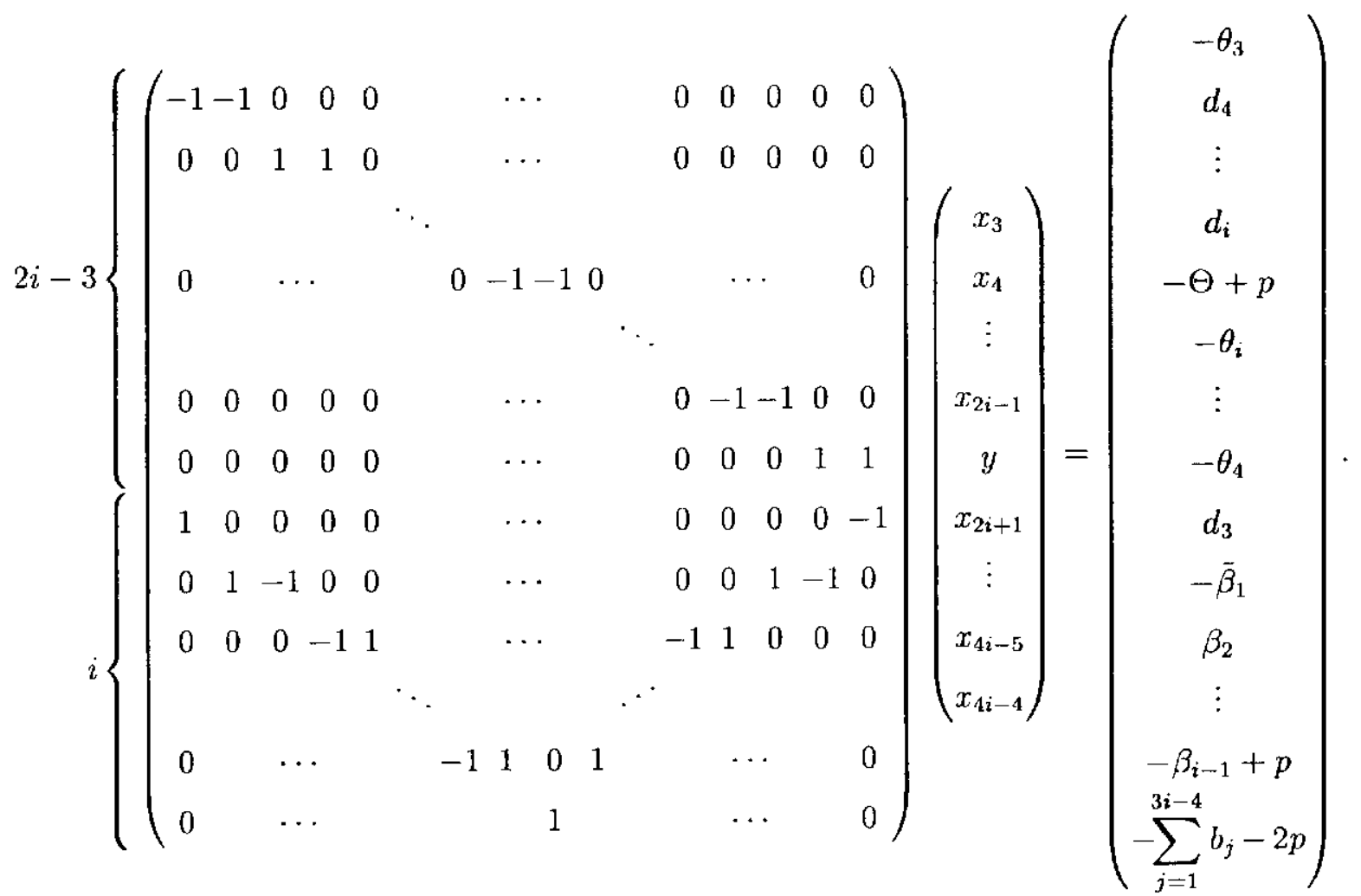

A matriz de coeficiente $A$ de $(3.43)$ é $(3 i-3) \times(4 i-6)$. O primeiro conjunto da partição é o mesmo que antes. Temos, para a $j$-ésima linha de $A$ e $j$-ésimo elemento do lado direito de $b$ de (3.43) no primeiro conjunto:

$$
\begin{aligned}
& A_{j}=\left\{\begin{array}{l}
(-1)^{j}\left(\boldsymbol{e}_{2 j-1}+\boldsymbol{e}_{2 j}\right), \quad \text { se } 1 \leq j \leq i-1 \\
(-1)^{j+1}\left(\boldsymbol{e}_{2 j-1}+\boldsymbol{e}_{2 j}\right), \quad \text { se } i \leq j \leq 2 i-3
\end{array},\right. \\
& b_{j}= \begin{cases}-\theta_{j+2}, \quad \text { se } 1 \leq j \leq i-2, j \text { ímpar } \\
d_{j+2}, & \text { se } 1 \leq j \leq i-2, j \text { par } \\
-\Theta+p, & \text { se } j=i-1 \\
-\theta_{2 i-j}, & \text { se } i \leq j \leq 2 i-3, j \text { par } \\
d_{2 i-j}, & \text { se } i \leq j \leq 2 i-3, j \text { impar }\end{cases}
\end{aligned}
$$


A equação redundante é adicionada ao segundo conjunto da partição, assim temos:

$$
\begin{aligned}
& A_{2 i-3+j}=\left\{\begin{array}{ll}
\boldsymbol{e}_{1}-\boldsymbol{e}_{4 i-6}, & \text { se } j=1 \\
(-1)^{j}\left(\boldsymbol{e}_{2 j \cdot 2}-\boldsymbol{e}_{2 j-1}+\boldsymbol{e}_{4 i-1 \cdot 2 j}-\boldsymbol{e}_{4 i-3-2 j}\right), & \text { se } 2 \leq j \leq i-2 \\
-\boldsymbol{e}_{2 i-4}+\boldsymbol{e}_{2 i-3}+\boldsymbol{e}_{2 i-1}, & \text { se } j=i-1 \\
\boldsymbol{e}_{2 i-2}, & \text { se } j=i
\end{array},\right. \\
& b_{2 i-3+j}= \begin{cases}-\tilde{\beta}_{1}, & \text { se } j=1 \\
(-1)^{j} \beta_{j}, & \text { se } 2 \leq j<i-1 \\
-\beta_{i-1}+p, & \text { se } j=i-1 \\
-\sum_{j=1}^{3 i-4} b_{j}-2 p, & \text { se } j=i\end{cases}
\end{aligned}
$$

Como os vetores de linha pertencem a $\mathbb{R}^{4 i-6}$, tomamos $\boldsymbol{e}_{k}$ como o $k$-ésimo vetor da base canônica deste espaço.

Seja $\mathcal{G}=(\mathcal{N}, \mathcal{A})$ o digrafo cuja matriz de incidência nó-arco é $A$. As cquações (3.43) representam um problema de fluxo paramétrico. Como o parâmetro $p$ é inteiro, o problema terá solução inteira não negativa se tiver solução não negativa. A estrutura de $\mathcal{G}$ é explicitada no próximo teorema. Ela ajudará a decompor (3.43) em um conjunto de sistemas menores, como feito na Subseçào 7.

Teorema 3.2.9 $O$ conjunto $\mathcal{A}$ pode ser particionado em $i-1$ subconjuntos. Destes, $i-2$ correspondem a ciclos não-direcionados de comprimento quatro em $\mathcal{G}$, e os restantes correspondem a um caminho não-direcionado. O j-ésimo subconjunto é o conjunto de arcos $\mathcal{A}_{j}$ no subgrafo $\mathcal{G}_{j}=\left(\mathcal{N}_{j}, \mathcal{A}_{j}\right)$ induzido pelos nós:

$$
\begin{array}{ll}
\text { Se } j \leq i-2: & \mathcal{N}_{j}=\{j, 2 i-2-j, 2 i-3+j, 2 i-2+j\} \\
\text { Se } j=i-1: & \mathcal{N}_{j}=\{i-1,3 i-4,3 i-3\}
\end{array}
$$

Demonstração: É suficiente mostrar que os subgrafos $\mathcal{G}_{j}$ têm a estrutura descrita e são arco-disjuntos, já que a soma das cardinalidades de $\mathcal{A}_{j}$, para $j=1, \ldots, i-1$, é $4(i-2)+2=4 i-6$, a cardinalidade de $\mathcal{A}$. Os elementos do lado direito correspondentes aos nós em $\mathcal{N}_{j}$ são dados na Tabela 3.2 . 


\begin{tabular}{|c|c|c|}
\cline { 2 - 3 } \multicolumn{1}{c|}{} & \multicolumn{2}{c|}{$1 \leq j \leq i-2$} \\
\cline { 2 - 3 } \multicolumn{1}{c|}{$j$ impar } & $j$ par \\
\hline$b_{j}$ & $-\theta_{j+2}$ & $d_{j+2}$ \\
\hline$b_{2 i-2-j}$ & $d_{j+2}$ & $\cdots \theta_{j+2}$ \\
\hline$b_{2 i-3+j}$ & $-\bar{\beta}_{1}$, se $j=1$ & $\beta_{j}$ \\
\hline & $-\beta_{j}$, se $j \geq 3$ & se $1 \leq j<i-2$ \\
$b_{2 i-2+j}$ & $\beta_{j+1}$ & $-\beta_{j+1}, \quad$ se $j=i-2$ \\
\hline
\end{tabular}

\begin{tabular}{|c|c|}
\cline { 2 - 2 } \multicolumn{1}{c|}{} & $j=i-1$ \\
\hline$b_{i-1}$ & $-\Theta+p$ \\
\hline$b_{3 i-4}$ & $-\beta_{i-1}+p$ \\
\hline$b_{3 i-3}$ & $-\sum_{j=1}^{3 i-4} b_{j}-2 p$ \\
\hline
\end{tabular}

Tabela 3.2: Demanda/suprimeto constantes para nós em $\mathcal{N}_{j}$

Da tabela é claro que os subgrafos $\mathcal{G}_{j}$ são arco-disjuntos já que

$$
\mathcal{N}_{\ell} \cap \mathcal{N}_{j}=\left\{\begin{array}{ll}
\{2 i-3+j\}, & \text { se } j=\ell+1 \\
\{2 i-2+j\}, & \text { se } j=\ell-1 \\
\emptyset, & \text { caso contrário }
\end{array} .\right.
$$

A submatriz de $A \mid b$ associada aus nós em $\mathcal{N}_{j}$ é

$$
\left(\begin{array}{c|c}
A_{j} & b_{j} \\
A_{2 i-2-j} & b_{2 i-2-j} \\
A_{2 i-3+j} & b_{2 i-3+j} \\
A_{2 i-2+j} & b_{2 i-2+j}
\end{array}\right) .
$$

Para $j=1$ a submatriz (3.44) torna-se:

$$
\left(\begin{array}{c|c}
-e_{1}-e_{2} & -\theta_{3} \\
e_{4 i-7}+e_{4 i-6} & d_{3} \\
e_{1}-e_{4 i-6} & -\bar{\beta}_{1} \\
e_{2}-e_{3}+e_{4 i-8}-e_{4 i-7} & \beta_{2}
\end{array}\right)
$$

Portanto $\mathcal{G}_{j}$, o subgrafo induzido por $\mathcal{N}_{j}$ tem matriz de incidencia

$$
\begin{aligned}
& \begin{array}{llll}
1 & 2 & 4 \mathrm{i}-7 & 4 \mathrm{i}-6
\end{array}
\end{aligned}
$$

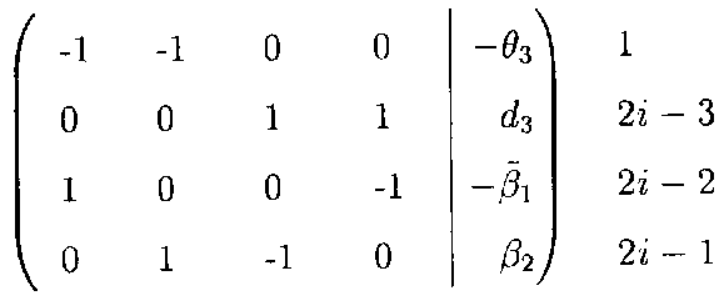


Se substituímos $j=1$ na Figura 3.13 , obtemos o subgrafo $\mathcal{G}_{1}$. Note a similaridade com a

Figura 3.11. Mostraremos que todos os subgrafos, exceto o último, têm esta estrutura.

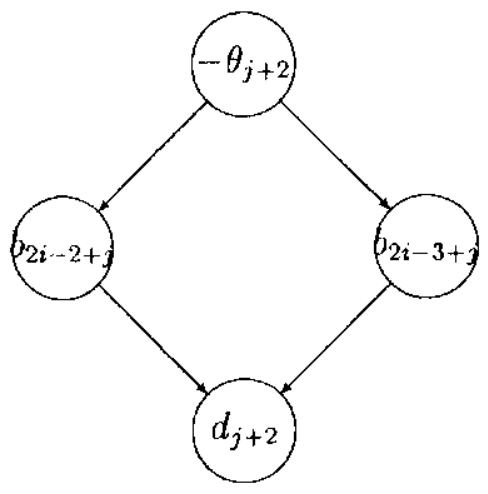

Figura 3.13: $O$ subgrafo $\mathcal{G}_{j}$ consiste dos ciclos não-orientados $\mathcal{C}_{j}$, para $j=1, \ldots, i-2$.

A submatriz (3.44), para $2 \leq j<i-2$, é dada por

$$
\begin{aligned}
& \left(\begin{array}{c|c}
(-1)^{j}\left(\boldsymbol{e}_{2 j-1}+\boldsymbol{e}_{2 j}\right) & b_{j} \\
(-1)^{2 i-1-j}\left(\boldsymbol{e}_{4 i-5-2 j}+\boldsymbol{e}_{4 i-4-2 j}\right) & b_{2 i \cdot 2 \cdots j} \\
(-1)^{j}\left(\boldsymbol{e}_{2 j-2}-\boldsymbol{e}_{2 j-1}+\boldsymbol{e}_{4 i-4-2 j}-\boldsymbol{e}_{4 i-3-2 j}\right) & (-1)^{j} \beta_{j} \\
(-1)^{j+1}\left(\boldsymbol{e}_{2 j}-e_{2 j+1}+\boldsymbol{e}_{4 i \cdot 62 j}-\boldsymbol{e}_{4 i \cdot 5-2 j}\right) & \left\{\begin{array}{cc}
(-1)^{j+1} \beta_{j+1}, & \text { se } j<i-2 \\
-\beta_{i-1}+p, & \text { se } j=i-2
\end{array}\right\}
\end{array}\right)
\end{aligned}
$$

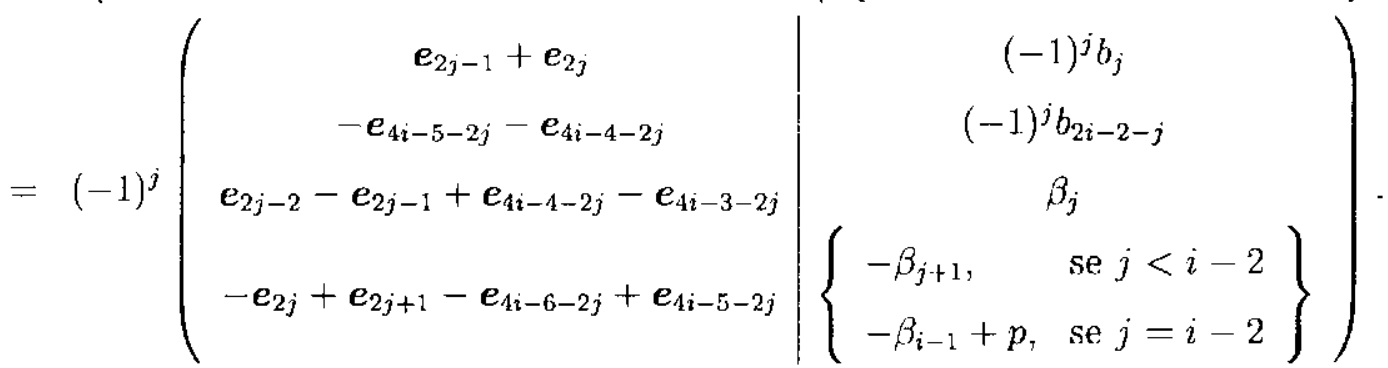

Assim a matriz de incidência de $\mathcal{G}_{j}$, para $j=2, \ldots, i-2$, é

$$
(-1)^{j}\left(\begin{array}{cccc|c}
2 \mathrm{j}-1 & 2 \mathrm{j} & 4 \mathrm{i}-5-2 \mathrm{j} & 4 \mathrm{i}-4-2 \mathrm{j} \\
1 & 1 & 0 & 0 & -1 \\
0 & 0 & -1 & 1 \\
-1 & 0 & 0 & 0 \\
0 & -1 & 1 & 0 & (-1)^{j} b_{j} \\
(-1)^{j} b_{2 i-2-j} \\
\beta_{j} \\
-\beta_{j+1}, & \text { se } j<i-2 \\
-\beta_{i-1}+p, & \text { se } j=i-2
\end{array}\right\} \begin{aligned}
& i \\
& 2 i-2-j \\
& 2 i-3+j \\
& 2 i-2+j
\end{aligned}
$$


Usando a Tabela 3.2 e a matriz acima concluímos que $\mathcal{G}_{j}$ tem a estrutura descjada.

Finalmente, consideremos submatriz de linhas associadas aos nós em $\mathcal{N}_{i-1}$ :

$$
\begin{aligned}
& \left(\begin{array}{c|c}
-e_{2 i-3}-e_{2 i-2} & -\Theta+p \\
-e_{2 i-4}+e_{2 i \cdot 3}+e_{2 i-1} & -\beta_{i-1}+p \\
\boldsymbol{e}_{2 i-2} & -\sum_{j=1}^{3 i-4} b_{j}-2 p
\end{array}\right)
\end{aligned}
$$

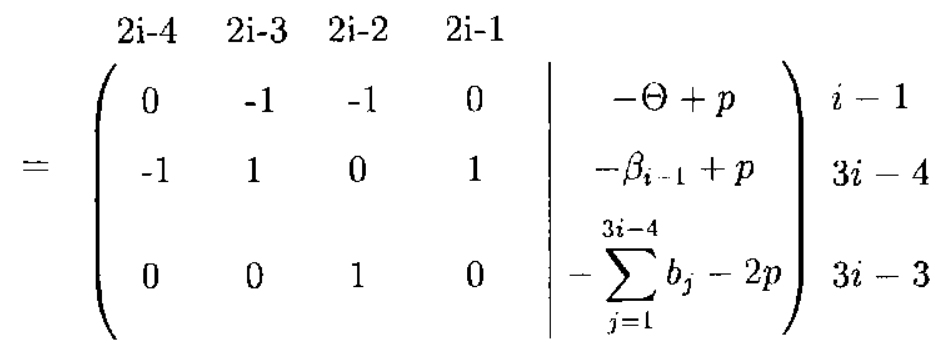

$$
\begin{aligned}
& \text { (-s) } \quad-\bar{\Theta} \stackrel{x_{2 i-1}}{y} \rightarrow \tilde{\beta}_{i-1} \\
& \text { Onde } \tilde{\Theta}=\Theta-p, \tilde{\beta}_{i-1}=\beta_{i-1}-p \text { e } s=\sum_{j=\mathbf{1}}^{3 i-4} b_{j}+2 p
\end{aligned}
$$

Figura 3.14: $\mathrm{O}$ subgrafo $\mathcal{G}_{i-1}$.

Logo o subgrafo $\mathcal{G}_{i-1}$, descrito na Figura 3.14 acima, contém somente dois arcos, associados às colunas $2 i-3$ e $2 i-2$ da matriz $A$ dada em (3.43).

O digrafo $\mathcal{G}$ é a união dos subgrafos $\mathcal{G}_{j}$, para $j=1, \ldots, i-1$. A Figura 3.15 dá uma idéia do digrafo inteiro. Os nós associados com o segundo conjunto de equações $2 i-1$, $\ldots, 3 i-4$, pode ser usado para dividir o sistema linear (3.43) em um conjunto equivalente de sistemas lineares menores, como feito na Subseção 7.

Teorema 3.2.10 O sistema linear (3.43) é equivalente a um conjunto de $i-1$ sistemas. O $j$-ésimo sistema neste conjunto, para $1 \leq j \leq i-2$, é dado por

$$
\left(\begin{array}{rrrr}
-1 & -1 & 0 & 0 \\
0 & 0 & 1 & 1 \\
1 & 0 & 0 & -1 \\
0 & 1 & -1 & 0
\end{array}\right)\left(\begin{array}{c}
w_{1}^{j} \\
w_{2}^{j} \\
w_{3}^{j} \\
w_{4}^{j}
\end{array}\right)=\left(\begin{array}{c}
-\theta_{j+2} \\
d_{j+2} \\
\hat{\beta}_{j} \\
-\hat{\beta}_{j}+\theta_{j+2}-d_{j+2}
\end{array}\right) \text {, }
$$




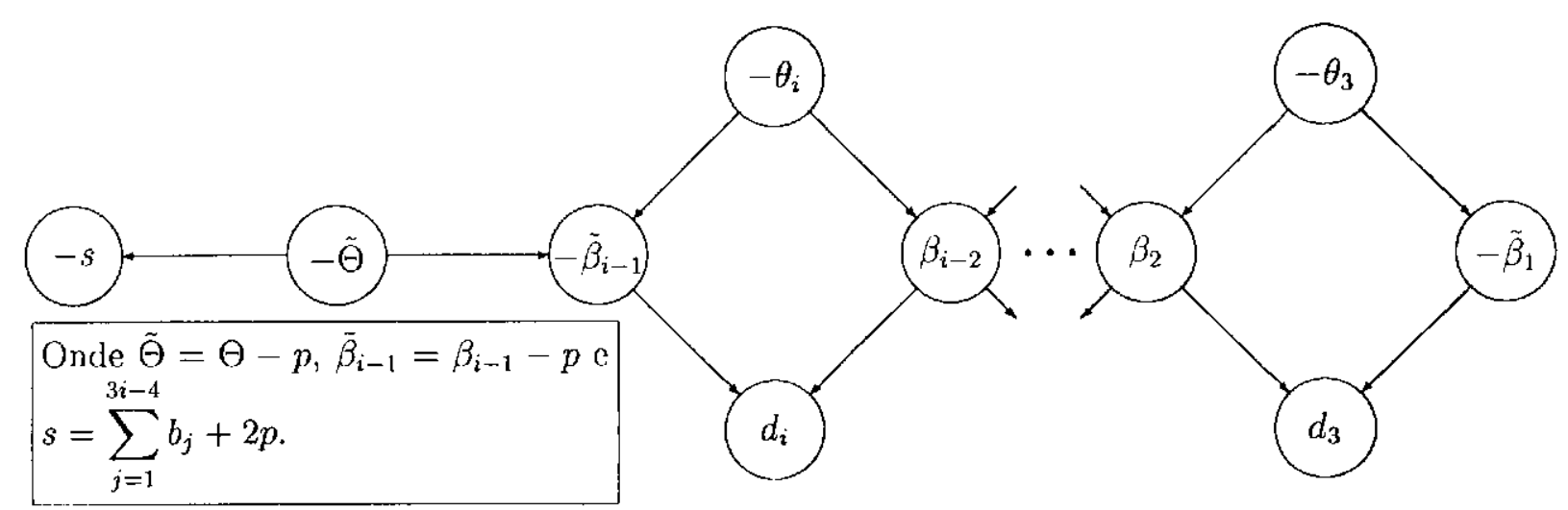

Figura 3.15: Estrutura do digrafo $\mathcal{G}$.

onde

$$
\hat{\beta}_{j}=\sum_{\ell=1}^{j}(-1)^{\ell} \beta_{\ell}-\sum_{\ell=1}^{j+1}\left(\theta_{\ell}-d_{\ell}\right), \quad \text { para } j=1, \ldots, i-2,
$$

e

$$
w^{j}=\left\{\begin{array}{ll}
\left(x_{2 j+1}, x_{2 j+2}, x_{4 i-3-2 j}, x_{4 i-2-2 j}\right), & \text { se } 1 \leq j \leq i-2 \text { é impar } \\
\left(x_{4 i-2-2 j}, x_{4 i-3-2 j}, x_{2 j+2}, x_{2 j+1}\right), & \text { se } 1 \leq j \leq i-2 \text { é par }
\end{array} .\right.
$$

$O(i-1)$-ésimo sistema é

$$
\left(\begin{array}{rr}
-1 & -1 \\
1 & 0 \\
0 & 1
\end{array}\right)\left(\begin{array}{c}
x_{2 i-1} \\
y
\end{array}\right)=\left(\begin{array}{c}
-\Theta+p \\
\hat{\beta}_{i-1}+p \\
-\sum_{j=1}^{3 i-4} b_{j}-2 p
\end{array}\right)
$$

onde

$$
\hat{\beta}_{i-1}=-\beta_{i-1}+\hat{\beta}_{i-2}-\theta_{i}+d_{i}
$$

Demonstração: $O$ digrafo $\mathcal{G}$ é a uniāo de $\mathcal{G}_{1}, \ldots, \mathcal{G}_{i-2}, \mathcal{G}_{i-1}$, onde os primeiros $i-2$ subgrafos são ciclos iguais aos da Figura 3.13 e o último é descrito na Figura 3.14. O procedimento de divisão usado na demonstração dada para o Teorema 3.2 .4 pode ser repetido, com resultados análogos, até a $(i-3)$-ésima divisão inclusive.

Isto é, no primeiro passo o nó de corte $2 i-1$ (com lado direito $\beta_{2}$ ) é usado para "separar" o subgrafo $\mathcal{G}_{1}$ do resto de $\mathcal{G}$. Algebricamente falando, o sistema linear (3.43) é dividido em dois pares de sistemas, um associado ao subgrafo $\mathcal{G}_{1}$ e o outro a $\cup_{j=2}^{i-1} \mathcal{G}_{j}$. O 
sistema associado a $\mathcal{G}_{1}$ é dado por (3.46), para $j=1$. Após $i-3$ tais divisões, teremos produzido um conjunto com $i-3$ sistemas lineares dados por (3.46) com $j=1, \ldots, i-3$, mais um sistema linear associado com o digrafo $\mathcal{G}_{i-2} \cup \mathcal{G}_{i-1}$, onde a constante associada ao nó de corte $\mathcal{G}_{i-3} \cap \mathcal{G}_{i-2}$ ć $\hat{\beta}_{i-2}$, satisfazendo (3.47). Assim, o sistema linear restante é

$$
\left(\begin{array}{rrrrrr}
1 & 1 & 0 & 0 & 0 & 0 \\
0 & 0 & -1 & -1 & 0 & 0 \\
0 & 0 & 0 & 0 & -1 & -1 \\
-1 & 0 & 0 & 0 & 0 & 1 \\
0 & -1 & 1 & 0 & 1 & 0 \\
0 & 0 & 0 & 1 & 0 & 0
\end{array}\right)\left(\begin{array}{c}
x_{2 i-3} \\
x_{2 i-2} \\
x_{2 i-1} \\
y \\
x_{2 i+1} \\
x_{2 i+2}
\end{array}\right)=\left(\begin{array}{c}
d_{i} \\
-\Theta+p \\
-\theta_{i} \\
\hat{\beta}_{i-2} \\
-\beta_{i-1}+p \\
-\sum_{j=1}^{3 i-4} b_{j}-2 p
\end{array}\right)
$$

Somando a primcira, terceira e quarta equaçōes de (3.51) à quinta, e reorganizando linhas e colunas, obtemos o seguinte sistema equivalente:

$$
\left(\begin{array}{rrrr|rr}
1 & 1 & 0 & 0 & 0 & 0 \\
0 & 0 & -1 & -1 & 0 & 0 \\
-1 & 0 & 0 & 1 & 0 & 0 \\
\hline 0 & 0 & 0 & 0 & -1 & -1 \\
0 & 0 & 0 & 0 & 1 & 0 \\
0 & 0 & 0 & 0 & 0 & 1
\end{array}\right)\left(\begin{array}{c}
x_{2 i-3} \\
x_{2 i-2} \\
x_{2 i+1} \\
x_{2 i+2} \\
x_{2 i-1} \\
y
\end{array}\right)=\left(\begin{array}{c}
d_{i} \\
-\theta_{i} \\
\hat{\beta}_{i-2} \\
-\Theta+p \\
-\beta_{i-1}+p+\hat{\beta}_{i-2}-\theta_{i}+d_{i} \\
-\sum_{j=1}^{3 i-4} b_{j}-2 p
\end{array}\right)
$$

O sistema acima é claramente decomponível ao longo das linhas exibidas em dois subsistemas: o primeiro equivale a (3.46) para $j=i-2$ (note que a última equação em (3.46) é redundante) e o segundo é precisamente (3.49), dado as fórmulas (3.47) e (3.50) para $\hat{\beta}_{i-2}$ e $\hat{\beta}_{i-1}$, respectivamente.

É importante notar que apenas duas componentes no vetor de incógnitas do sistema parametrizado (3.43), ou seja, $x_{2 i-1}$ e $y$, dependerão do parâmetro $p$ (o valor de $x_{2 i}$ ), já que este parâmetro aparece somente no subsistema (3.49). Logo a solução de (3.38) foi subdividida em $(i-1)$ 4-uplas e uma 3-upla. A primeira 4-upla, $q_{1}=\left(x_{1}, x_{2}, x_{4 i-3}, x_{i i-2}\right)$ tem valor único $\left(\theta_{1}, d_{2}-\theta_{1}, \theta_{2}-d_{1}, d_{1}\right)$. A $j$-ésima 4-upla, para $2 \leq j \leq i-1, q_{j}=$ 
$\left(x_{2 j-1}, x_{2 j}, x_{4 i-1-2 j}, x_{4 i-2 j}\right)$, é a reordenação apropriada da solução do sistema linear

$$
\left(\begin{array}{rrrr}
-1 & -1 & 0 & 0 \\
0 & 0 & 1 & 1 \\
1 & 0 & 0 & -1 \\
0 & 1 & -1 & 0
\end{array}\right)\left(\begin{array}{c}
w_{1}^{j-1} \\
w_{2}^{j-1} \\
w_{3}^{j-1} \\
w_{4}^{j-1}
\end{array}\right)=\left(\begin{array}{c}
-\theta_{j+1} \\
d_{j+1} \\
\hat{\beta}_{j-1} \\
-\hat{\beta}_{j-1}+\theta_{j+1}-d_{j+1}
\end{array}\right)
$$

Pelo Lema 3.2.5, $w^{j-1}=\left(\hat{\beta}_{j-1}, \theta_{j+1}-\hat{\beta}_{j-1}, d_{j+1}, 0\right)+\alpha(1,-1,-1,1)$, para $j=2, \ldots$, $i-1$, e o Lema 3.2.6 dá condições necessárias e suficientes para uma tal solução ser inteira não negativa, bem como o número de soluções. A última parte do "quebra-cabeça" é $q_{i}=\left(x_{2 i-1}, y, x_{2 i}\right)$. O Sistema (3.49) tem uma solução se, e somente se,

$$
-\Theta+p+\hat{\beta}_{i-1}+p-\sum_{j=1}^{3 i-4} b_{j}-2 p=0
$$

Mas, como a condição acima é, por construção, sempre satisfeita, concluímos que $q_{i}=$ $\left(\hat{\beta}_{i-1}+p,-\sum_{j=1}^{3 i-4} b_{j}-2 p, p\right)$. Este útimo vetor deve também satisfazer condiçōes de não negatividade e integralidade. Agora $q_{i}$ ć intciro se, e somente se, $p$ é inteiro, já que todos os dados do problema são inteiros. As condições de não negatividade são equivalentes as três desigualdades:

$$
\begin{aligned}
p>-\hat{\beta}_{i-1} \\
\frac{-\sum_{j=1}^{3 i-4} b_{j}}{2}=\frac{\Theta-\hat{\beta}_{i-1}}{2} \geq \\
p \geq 0
\end{aligned}
$$

Eliminando $p$ com o método de Fourier-Motzkin conseguimos

$$
\begin{aligned}
\Theta & \geq-\hat{\beta}_{i-1} \\
\Theta & \geq \hat{\beta}_{i-1}
\end{aligned}
$$

Assirn (3.38) tem uma solução inteira não negativa se, e somente se, as seguintes 
condiçōes são satisfeitas:

$$
\left\{\begin{aligned}
d_{2} & \geq \theta_{1} \\
\theta_{2} & \geq d_{1} \\
\theta_{j+1} & \geq \hat{\beta}_{j-1}=\sum_{\ell=1}^{j-1}(-1)^{\ell} \beta_{\ell}-\sum_{\ell=1}^{j}\left(\theta_{\ell}-d_{\ell}\right), \quad \text { para } j=2, \ldots, i-1 \\
d_{j+1} & \geq-\hat{\beta}_{j-1}=-\sum_{\ell=1}^{j-1}(-1)^{\ell} \beta_{\ell}+\sum_{\ell=1}^{j}\left(\theta_{\ell}-d_{\ell}\right), \text { para } j=2, \ldots, i-1 \\
\Theta & \geq-\sum_{\ell=1}^{i-1}(-1)^{\ell} \beta_{\ell}+\sum_{\ell=1}^{i}\left(\theta_{\ell}-d_{\ell}\right) \\
\Theta & \geq \sum_{\ell=1}^{i-1}(-1)^{\ell} \beta_{\ell}-\sum_{\ell=1}^{i}\left(\theta_{\ell}-d_{\ell}\right)
\end{aligned}\right.
$$

O número de soluções é dado pelo produto $\prod_{j=2}^{i} n_{j}$, onde $n_{j}$ é o número de intciros no intervalo $\left[\max \left\{0,-\hat{\beta}_{j-1}\right\}, \min \left\{\theta_{j+1}-\hat{\beta}_{j-1}, d_{j+1}\right\}\right]$, para $j=2, \ldots, i-1$, e $n_{i}$ é o número de inteiros no intervalo $\left[\max \left\{0,-\hat{\beta}_{i-1}\right\},\left(\Theta-\hat{\beta}_{i-1}\right) / 2\right]$.

\subsubsection{Caso $\mathrm{n}=2 \bmod 4$}

\section{O sistema linear}

Agora vamos assumir $n=2 i$, onde $i \geq 3$ é ímpar. O problema é encontrar uma soluçāo inteira não negativa do sistema linear: 


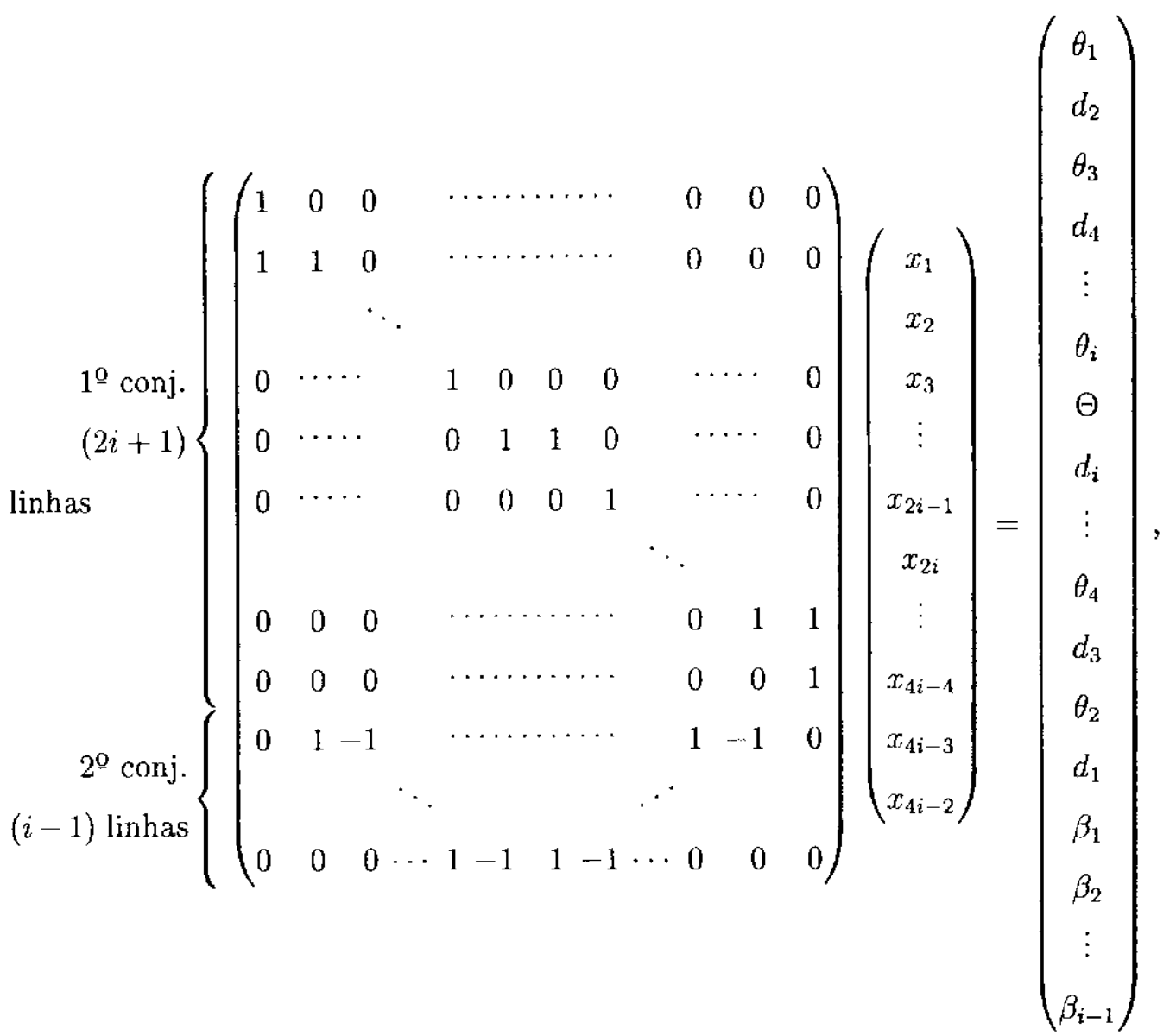

onde $\Theta, \theta_{j}$ e $d_{j}$, para $j=1, \ldots, i$, são inteiros positivos e $\beta_{j}$, para $j=1, \ldots, i-1$, são inteiros. A matriz de coeficientcs $A$ de $(3.53)$ é $3 i \times(4 i-2)$, e suas linhas são particionadas em dois conjuntos, o primeiro contendo as linhas 1 até $2 i+1$ e, o segundo, linhas $2 i+2$ até $3 i$. O lado direito $\bar{b}$ e as equações são particionadas da mesma forma. As fórmulas para a $j$-ésima linha do primeiro grupo e o elemento correspondente do lado direito são

$$
\bar{A}_{j}= \begin{cases}e_{1}, & \text { se } j=1 \\ e_{2 j-3}+e_{2 j-2}, & \text { se } 2<j<2 i \\ e_{2 j-4}, & \text { se } j=2 i+1\end{cases}
$$


$\mathrm{c}$

$$
\bar{b}_{j}= \begin{cases}\theta_{j}, & \text { se } 1 \leq j \leq i, j \text { impar } \\ d_{j}, & \text { se } 1 \leq j \leq i, j \text { par } \\ \Theta, & \text { se } j=i+1 \\ \theta_{2 i+2-j}, & \text { se } i+2 \leq j \leq 2 i+1, j \text { par } \\ d_{2 i+2-j}, & \text { se } i+2 \leq j \leq 2 i+1, j \text { ímpar }\end{cases}
$$

onde $\boldsymbol{e}_{j}$ é o $j$-ćsimo vetor da base canònica $\mathbb{R}^{4 i-2}$. No segundo grupo, a $j$-ésima linha e respectivo lado direito são como segue

$$
\widetilde{A}_{2 i+1+j}=e_{2 j}-e_{2 j+1}+e_{4 i \cdot 2 \cdot 2 j}-e_{4 i-1-2 j}, \text { se } 1 \leq j \leq i-1
$$

$\mathrm{e}$

$$
\bar{b}_{2 i+1+j}=\beta_{j}, \text { se } 1 \leq j \leq i-1 \text {. }
$$

Seguiremos a abordagem adotada nos casos anteriores estudados. Nossa exposição será bem sucinta, já que esta é a terceira vez que esta abordagem é usada. O primeiro passo é eliminar as variáveis $x_{1}, x_{2}, x_{4 i-2}$ e $x_{4 i-3}$, para reduzir o sistema. As equações usadas e os valores obtidos são os mesmos como na Subseção 3.2.2. Mais uma vez obtemos o conjunto de desigualdades (3.10), surgidas inicialmente na Subseção 3.2.1, condições necessárias e suficientes para a solução parcial $\left(x_{1}, x_{2}, x_{4 i-2}, x_{4 i-3}\right)=\left(\theta_{1}, d_{2}-\theta_{1}, \theta_{2}-d_{1}, d_{1}\right)$ ser não negativa. A próxima subseção trata do sistema reduzido. 


\section{O sistema reduzido e suas soluçōes}

Eliminando as variáveis $x_{1}, x_{2}, x_{4 i-3}$ e $x_{4 i-2}$ e as equações $1,2,2 i+1$ e $2 i$ o sistema reduzido (3.54) é obtido de (3.53):

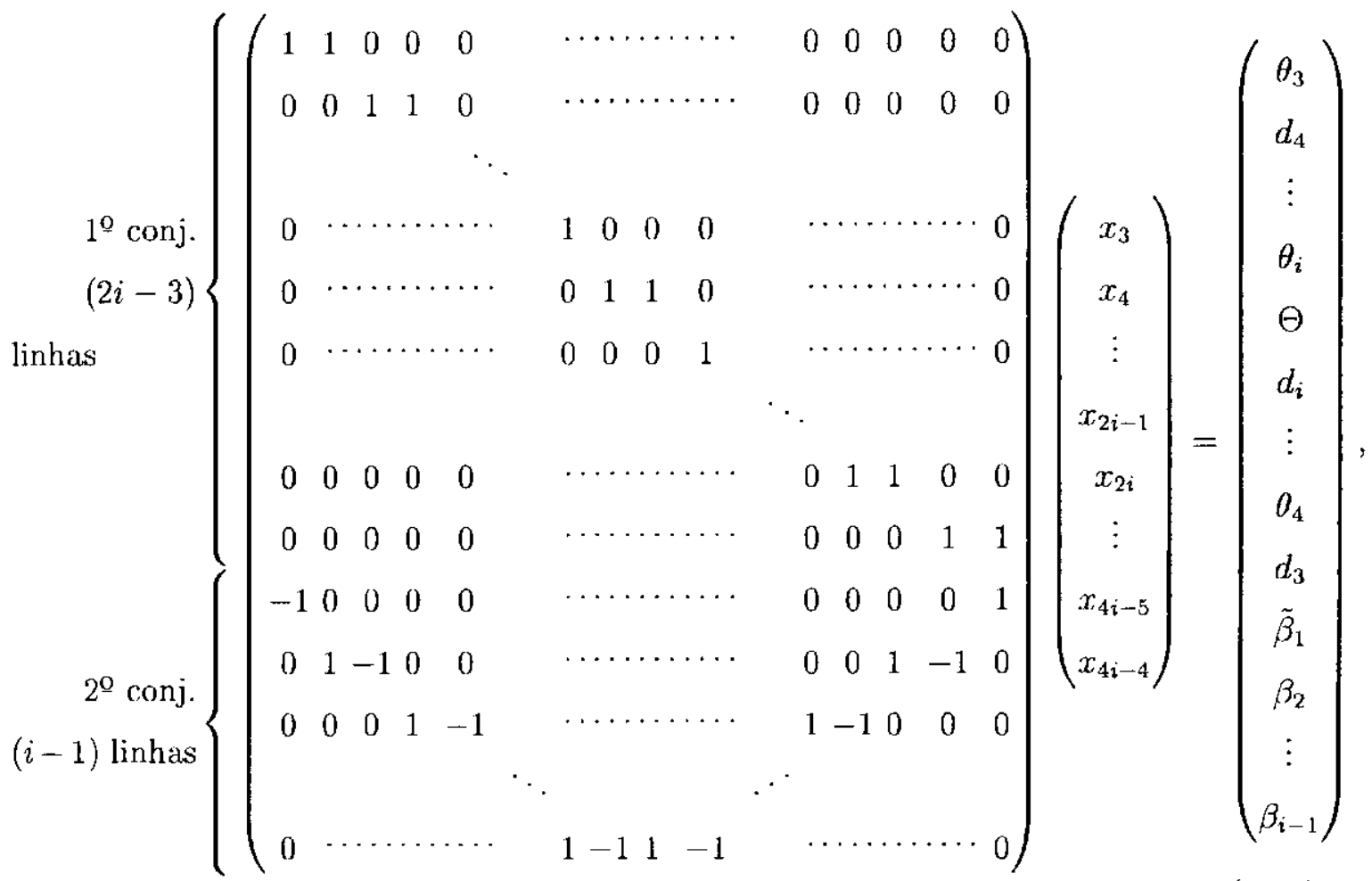

onde $\tilde{\beta}_{1}=\beta_{1}+\theta_{1}+\theta_{2}-d_{1}-d_{2}$. Este sistema reduzido tem $3 i-4$ equações e $4 i-6$ incógnitas.

O sistema (3.54) tem matriz de coeficientes $\bar{A}$ e o lado direito $\overline{\bar{b}}$, que herdam a partição de $\bar{A}$ e $\bar{b}$. A j-ésima linha e o elemento do lado direito no primeiro conjunto são dados por

$$
\overline{\bar{A}}_{j}=e_{2 j-1}+e_{2 j}, \quad \text { para } j=1, \ldots, 2 i-3
$$

e

$$
\overline{\bar{b}}_{j}= \begin{cases}\theta_{j+2}, & \text { se } 1 \leq j \leq i-2, j \text { ímpar } \\ d_{j+2}, & \text { se } 1<j \leq i-2, j \text { par } \\ \Theta, & \text { se } j=i-1 \\ \theta_{2 i-j}, & \text { se } i \leq j \leq 2 i-3, j \text { ímpar } \\ d_{2 i-j}, & \text { se } i \leq j<2 i-3, j \text { ímpar }\end{cases}
$$


No segundo conjunto, as fórmulas sāo:

$$
\overline{\bar{A}}_{2 i-3+j}= \begin{cases}-\boldsymbol{e}_{1}+\boldsymbol{e}_{4 i-6}, & \text { se } j=1 \\ e_{2 j-2}-e_{2 j-1}+\boldsymbol{e}_{4 i-4-2 j}-\boldsymbol{e}_{4 i-3-2 j}, & \text { se } 2 \leq j \leq i-1\end{cases}
$$

$\mathrm{e}$

$$
\overline{\bar{b}}_{2 i-3+j}=\left\{\begin{array}{l}
\tilde{\beta}_{1}, \quad \text { se } j=1 \\
\beta_{j}, \quad \text { se } 2 \leq j \leq i-1
\end{array} .\right.
$$

Note que $\boldsymbol{e}_{j}$ é agora $j$-ćsimo vetor da base canônica de $\mathbb{R}^{4 i-6}$.

Lema 3.2.11 Cada coluna de $\overline{\bar{A}}$, contém exatamente dois coeficientes não nulos, um no primeiro conjunto de linhas e o outro no segundo conjunto.

Demonstração: A demonstração é análoga à demonstração dos Lemas 3.2.1 e 3.2.7.

Multiplicando por -1 a $j$-ésima equação de (3.54), onde $1 \leq j \leq i-2$ é ímpar e $i-1 \leq j \leq 3 i-4$ é par, obtemos o sistema equivalente.

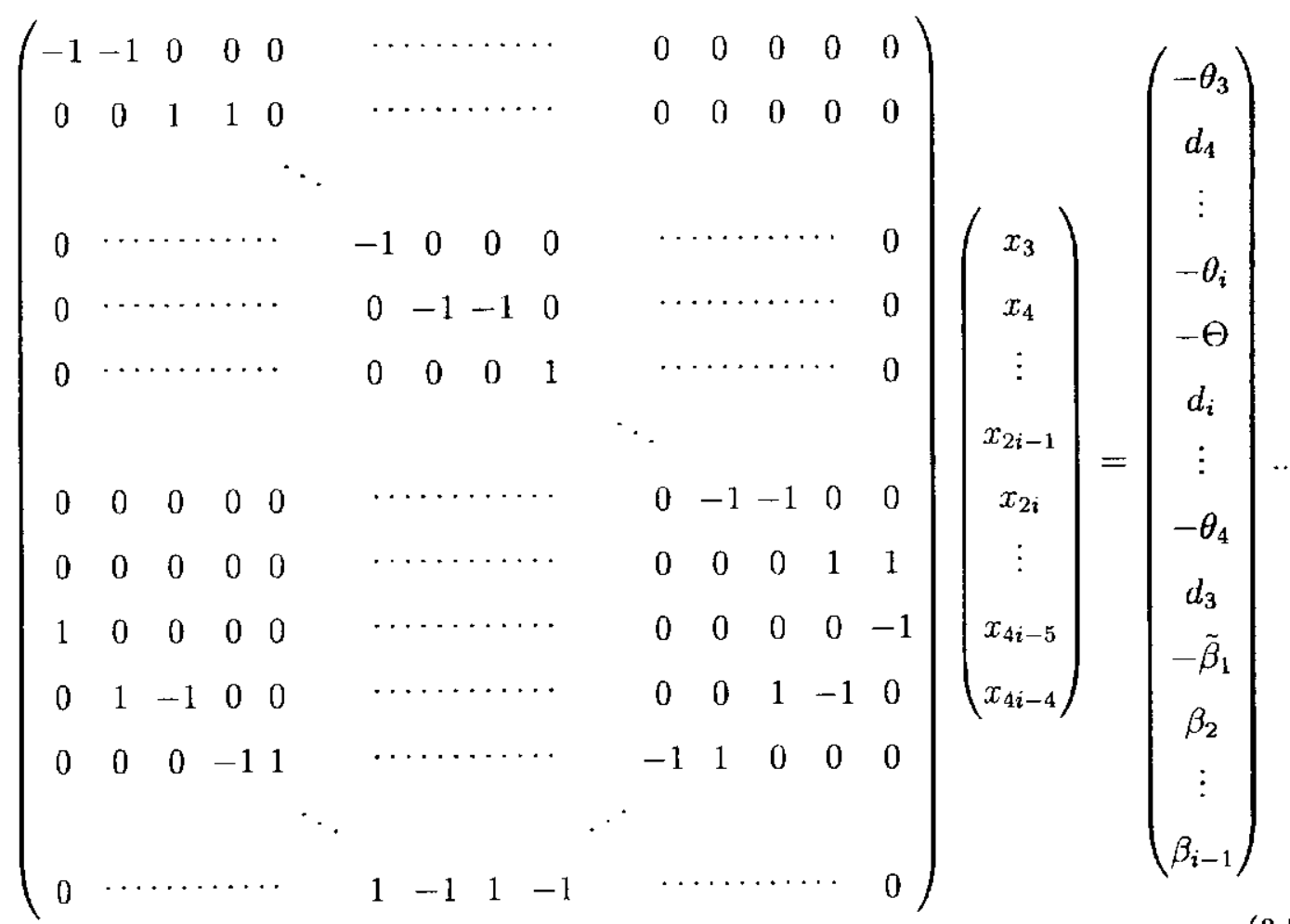

As fórmulas para a j-ésima linha e elementos do lado direito devem ser adaptados adequadamente. Mantendo a partição e denotando por $A$ e $b$, respectivamente, a matriz de 
coeficientes e o vetor do lado direito (3.55), temos, para o primeiro conjunto:

$$
\begin{aligned}
& A_{j}=\left\{\begin{array}{ll}
(-1)^{j}\left(e_{2 j-1}+e_{2 j}\right), \quad \text { se } 1 \leq j \leq i-2 \\
(-1)^{j+1}\left(e_{2 j-1}+e_{2 j}\right), \quad \text { se } i-1<j \leq 2 i-3
\end{array},\right. \\
& b_{j}= \begin{cases}-\theta_{j+2}, & \text { se } 1 \leq j \leq i-2, j \text { impar } \\
d_{j+2}, & \text { se } 1<j \leq i-2, j \text { par } \\
-\Theta, & \text { se } j=i-1 \\
-\theta_{2 i-j}, & \text { se } i<j \leq 2 i-3, j \text { par } \\
d_{2 i-j}, & \text { se } i<j \leq 2 i-3, j \text { impar }\end{cases}
\end{aligned}
$$

No segundo conjunto, as fórmulas são:

$$
\begin{aligned}
A_{2 i-3+j} & = \begin{cases}e_{1}-e_{4 i-6}, & \text { se } j=1 \\
(-1)^{j}\left(e_{2 j-2}-e_{2 j-1}+e_{4 i-4-2 j}-e_{4 i-3-2 j}\right), & \text { se } 2 \leq j \leq i-1\end{cases} \\
b_{2 i-3+j} & =\left\{\begin{array}{ll}
-\tilde{\beta}_{1}, & \text { se } j=1 \\
(-1)^{j} \beta_{j}, & \text { se } 2 \leq j \leq i-1
\end{array} .\right.
\end{aligned}
$$

Teorema 3.2.12 O sistema (3.55) é equivalente ao conjunto de $i-1$ sistemas menores. O j-ésimo sistema, para $1 \leq j \leq i-2$, é dado por

$$
\left(\begin{array}{rrrr}
-1 & -1 & 0 & 0 \\
0 & 0 & 1 & 1 \\
1 & 0 & 0 & -1 \\
0 & 1 & -1 & 0
\end{array}\right)\left(\begin{array}{c}
w_{1}^{j} \\
w_{2}^{j} \\
w_{3}^{j} \\
w_{4}^{j}
\end{array}\right)=\left(\begin{array}{c}
-\theta_{j+2} \\
d_{j+2} \\
\hat{\beta}_{j} \\
-\hat{\beta}_{j}+\theta_{j+2}-d_{j+2}
\end{array}\right),
$$

onde

$$
\hat{\beta}_{j}=\sum_{\ell=1}^{j}(-1)^{\ell} \beta_{\ell}-\sum_{\ell=1}^{j+1}\left(\theta_{\ell}-d_{\ell}\right), \quad \text { para } j=1, \ldots, i-2,
$$

$e$

$$
w^{j}= \begin{cases}\left(x_{2 j+1}, x_{2 j+2}, x_{4 i-3-2 j}, x_{4 i-2-2 j}\right), & \text { se } 1 \leq j \leq i-2 \text { é impar } \\ \left(x_{4 i-2-2 j}, x_{4 i-3-2 j}, x_{2 j+2}, x_{2 j+1}\right), & \text { se } 1 \leq j \leq i-2 \text { é par }\end{cases}
$$

O sistema $i-1$, o último, é dado por

$$
\left(\begin{array}{cc}
-1 & -1 \\
-1 & 1
\end{array}\right)\left(\begin{array}{c}
x_{2 i-1} \\
x_{2 i}
\end{array}\right)=\left(\begin{array}{c}
-\Theta \\
\hat{\beta}_{i-1}
\end{array}\right)
$$

onde

$$
\hat{\beta}_{i-1}=\sum_{\ell=1}^{i-1}(-1)^{\ell} \beta_{\ell}-\sum_{\ell=1}^{i}\left(\theta_{\ell}-d_{\ell}\right)
$$


Demonstração: Aplicando precisamente a mesma decomposição que foi usada nas provas dos Teoremas 3.2.4 e 3.2.10 para (3.55), obtemos $(i-2)$ problemas de fluxos em ciclos não direcionados de comprimento quatro, do tipo (3.56). O sistema restante é (3.59).

O sistema (3.59) tem solução única $\left(x_{2 i-1}, x_{2 i}\right)=\left(\left(\Theta-\hat{\beta}_{i-1}\right) / 2,\left(\Theta+\hat{\beta}_{i-1}\right) / 2\right)$. Este subvetor de $x$ é não negativo e inteiro se, e somente se, as seguintes condições se mantêm:

$$
\begin{aligned}
& \Theta-\hat{\beta}_{i-1} \text { é par } \\
& \Theta \geq \hat{\beta}_{i-1} \\
& \Theta \geq-\hat{\beta}_{i-1}
\end{aligned}
$$

A decomposição fornecida pelo Teorema 3.2.12 imediatamente leva a um conjunto de condições para a existência de valores inteiros năo negativos para as componentes restantes de $x$. O conjunto de todas as condições resultam no sistema (3.52) juntamente com a restrição que $\Theta-\hat{\beta}_{i, 1}$ seja par. O número de soluções é dado pelo produto $\prod_{j=2}^{i-1} n_{j}$, onde $n_{j}$ é o número de inteiros no intervalo $\left[\max \left\{0,-\hat{\beta}_{j-1}\right\}, \min \left\{\theta_{j+1}-\hat{\beta}_{j-1}, d_{j+1}\right\}\right]$, para $j=2, \ldots, i-1$.

\subsection{Conclusão}

Nesta seção o teorema principal é provado, estabelecendo a correspondência entrc os sistemas lineares obtidos dos algoritmos na Seçāo 3.1 e a álgebra na Seção 3.2.

Prova do Teorema 3.0.1..

É fácil ver que os sistemas lineares (3.5), (3.7) e (3.8) obtidos do algoritmo na Scção 3.1 são representados pelas seguintes matrizes. 
Caso de dimensão ímpar:

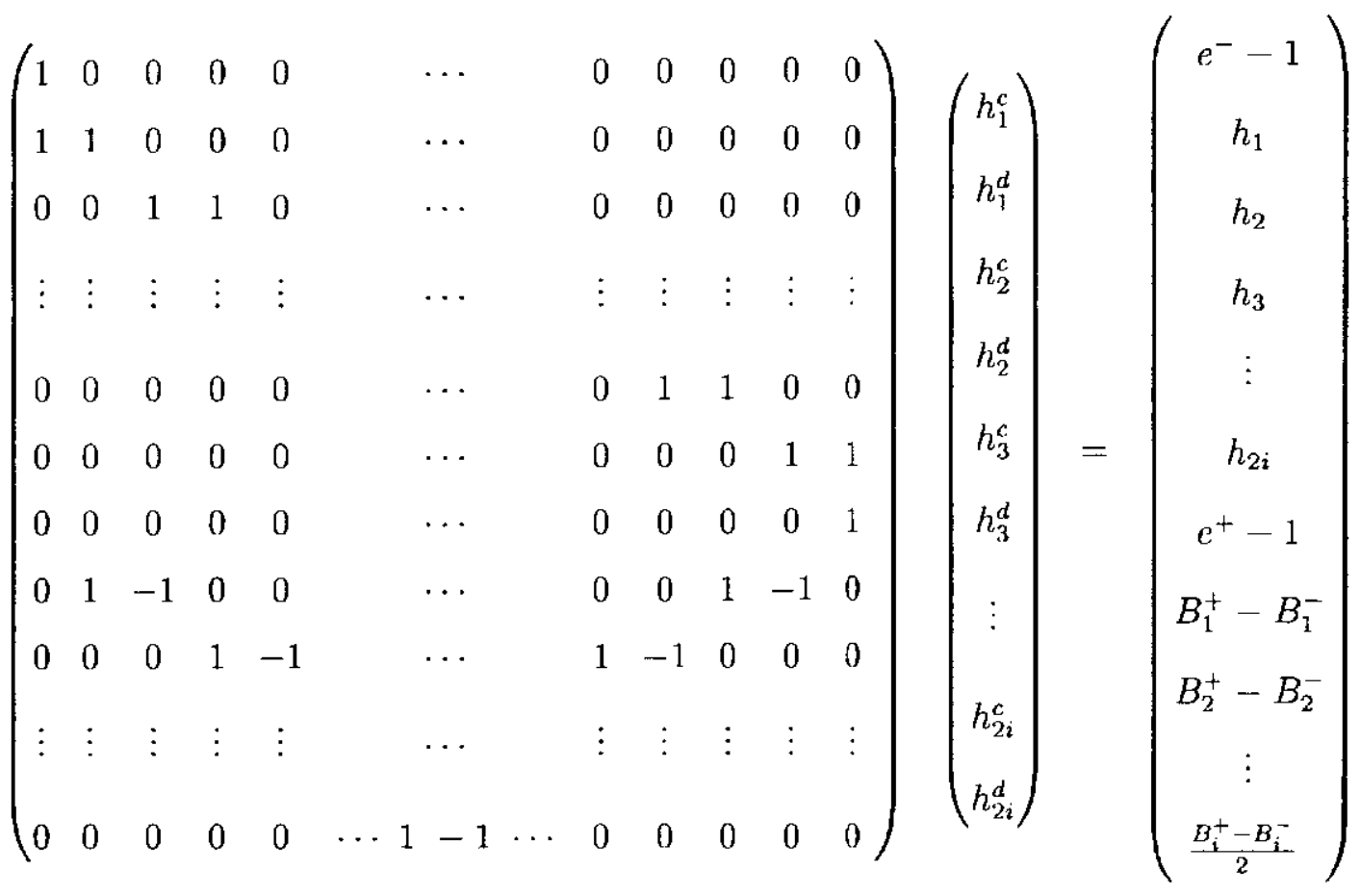

Caso de dimensão par $n=0 \bmod 4$ :

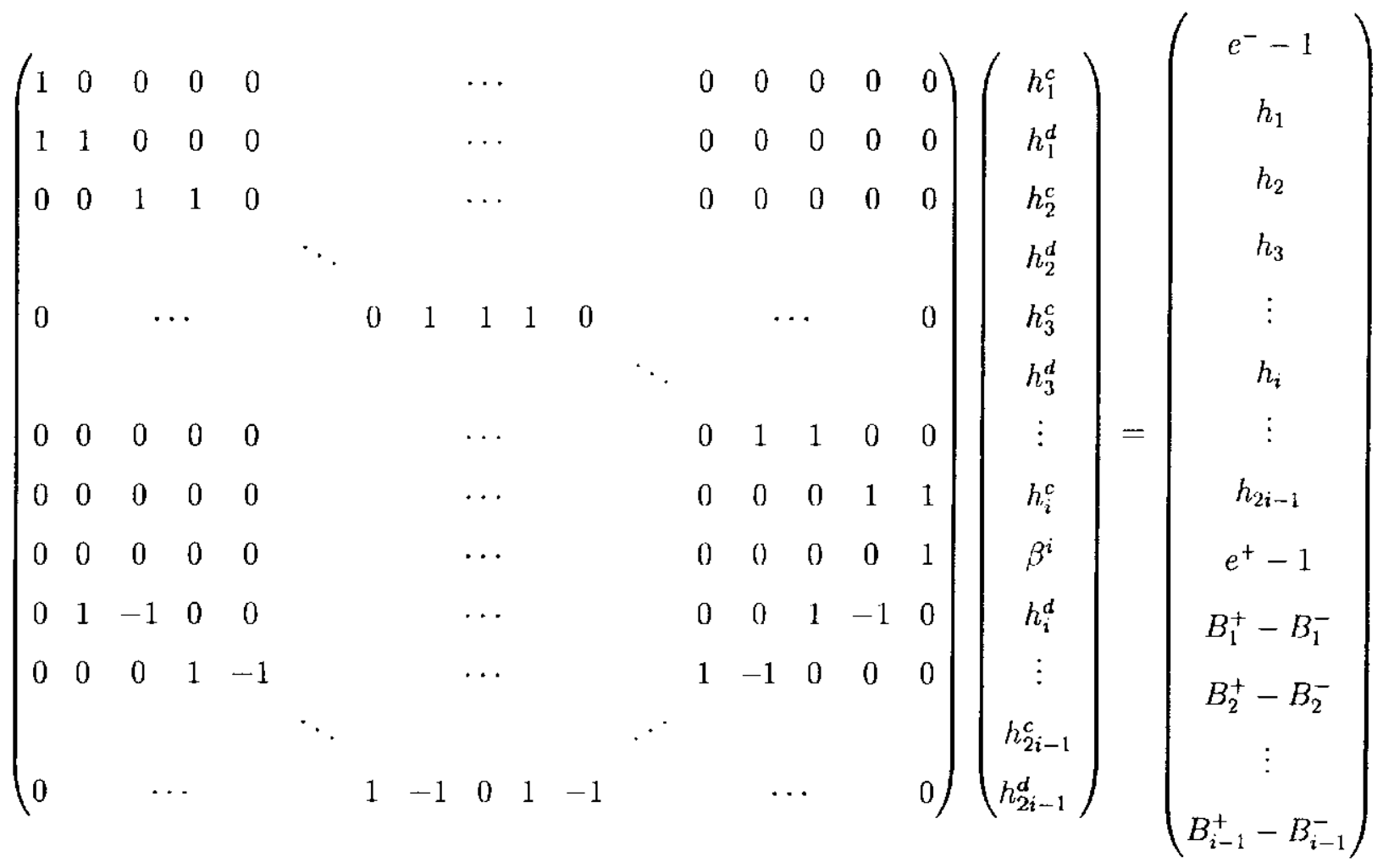


Caso de dimensão par $n=2 \bmod 4$ :

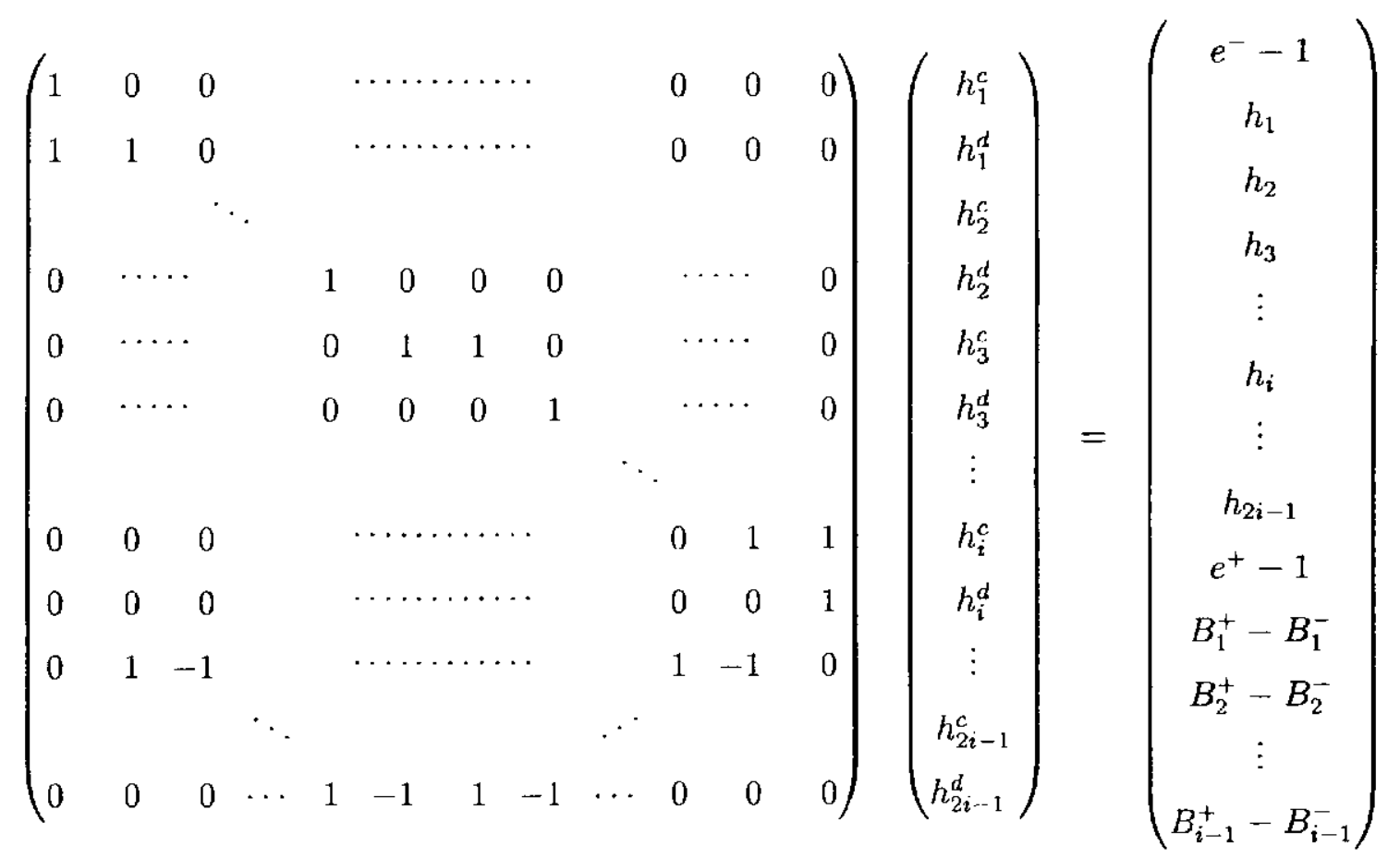

Estas matrizes correspondem a (3.9), (3.38) e (3.53) respectivamente, na Seção 3.2. Naquela seção condições necessárias e suficientes foram provadas para a existência de soluçōes inteiras não negativas para estes sistemas. Estas condições são resumidas na Seção 3.2 no caso de dimensão ímpar em (3.37), no caso 0 mod 4 em (3.52) e no caso $2 \bmod 4 \mathrm{em}(3.52)$ e (3.60). Fistas condiçōes correspondem as desigualdades de PoincaréHopf (2.17), (2.18) e (2.20) onde $h_{n}$ e $h_{0}$ são tomados iguais a zero.

Logo as desigualdades de Poincaré-Hopf (2.17), (2.18) e (2.20) são condições necessárias e suficientes para um vértice explodir. A continuação do grafo abstrato de Lyapunov ocorrerá se esta condição é verificada para cada vértice. 


\section{Capítulo 4}

\section{Desigualdades de Poincaré-Hopf e Desigualdades de Morse}

Neste capítulo mostraremos que as desigualdades de Poincaré-Hopf (2.21)-(2.23) são satisfeitas, se e somente se, as desigualdades de Morse são satisfeitas (1) para algum vetor de números de Betti $\left(\gamma_{0}, \ldots, \gamma_{n}\right)$.

Vamos estabelecer o teorema principal deste capítulo em tcrmos de uma coleção de números não negativos $\left(h_{0}, h_{1} \ldots, h_{n}, \kappa\right)$, com $\kappa=0$.

Teorema 4.0.1 Um conjunto de números não negativos $\left(h_{0}, h_{1} \ldots, h_{n}\right)$ satisfaz as desigualdades de Poincaré-Hopf em (2.21) se, e somente se, satisfaz as desigualdades de Morse (1) para algum vetor de números de Betti $\left(\gamma_{0}, \gamma_{1}, \ldots, \gamma_{n-1}, \gamma_{n}\right)$.

O passo crucial na prova do teorema principal é definir um sistema linear, chamado sistema $h^{\text {cd }}$ reduzido que pode ser caracterizado pelas dimensões do índice de Conley homológico $\left(h_{0}, \ldots, h_{n}\right)$ e cujas incógnitas são precisamente $\left(h_{1}^{c}, h_{1}^{d}, \ldots, h_{n-1}^{c}, h_{n-1}^{d}\right)$. Soluçōes não negativas para estes sistemas correspondem a diferentes formas que podemos escolher $h_{j}^{c}$ e $h_{j}^{d}$ para dados de índice pré-fixados $\left(h_{0}, \ldots, h_{n}\right)$. No Capítulo 3 foi mostrado que o sistema $h^{\text {cd }}$ tem uma solução inteira não negativa se, e somente sc, as desigualdades de Poincaré-Hopf (2.21), (2.22) e (2.23) são satisfeitas. Também mostramos neste mesmo capítulo que este sistema $h^{\text {cl }}$ constitui um problema de fluxo em rede, com possíveis restrições adicionais. A natureza da redc cnvolvida permite uma caracterização completa de todas as soluções possíveis do sistema $h^{\text {cd }}$ por meio da solução particular do sistema 
(um ponto extremo, ou vértice, do politopo associado com o sistema $h^{c d}$ ) e as circulações simples da rede (soluções elementares da versão homogênea do sistema $h^{\text {cd }}$ ).

A novidade aqui é que o $h_{j}^{c}$ e $h_{j}^{d}$ serão usados para definir um vetor de números de Betti que satisfaz as desigualdades de Morse para dados de índice pré-fixados $\left(h_{0}, \ldots, h_{n}\right)$. Considerando uma solução particular e todas as possiveis combinações de circulações, podemos construir o casco convexo de todos os vetores de números de Betti que satisfazem as desigualdades de Morse para um dado de índice pré-fixado. O casco convexo da coleção de vetores de números de Betti que satisfazem as desigualdades de Morse constitui um politopo. O politopo de Morse $\mathcal{P}\left(h_{0}, \ldots, h_{n}\right)$ (ou simplesmente $\mathcal{P}$, se consideramos um genérico fixado $\left.\left(h_{0}, \ldots, h_{n}\right)\right)$ é a intersecção deste casco convexo com o quadrante não negativo.

Este capítulo é dividido em duas seções. Na Seção 4.1 os principais resultados de equivalência sāo estabelecidos, isto é, o sistema $h^{\text {cd }}$ admite soluções inteiras não negativas se, e somente se, existem vetores de números de Betti inteiros não negativos que satisfazem as desigualdades de Morse. Na Seção 4.2 descrevemos o politopo de Morse $\mathcal{P}$ e apresentamos propriedades geométricas adicionais.

\subsection{Resultados de equivalência}

No Capítulo 3 foi mostrado que

Proposição 4.1.1 Os sistemas (4.1) e (4.2) têm solução inteira não negativas $\left(h_{1}^{c} h_{1}^{d}, \ldots, h_{n-1}^{c} h_{n-1}^{d}\right)$ se, e somente se, as desigualdades de Poincaré-Hopf (2.20), (2.7), (2.16), para blocos isolantes são satisfeitas.

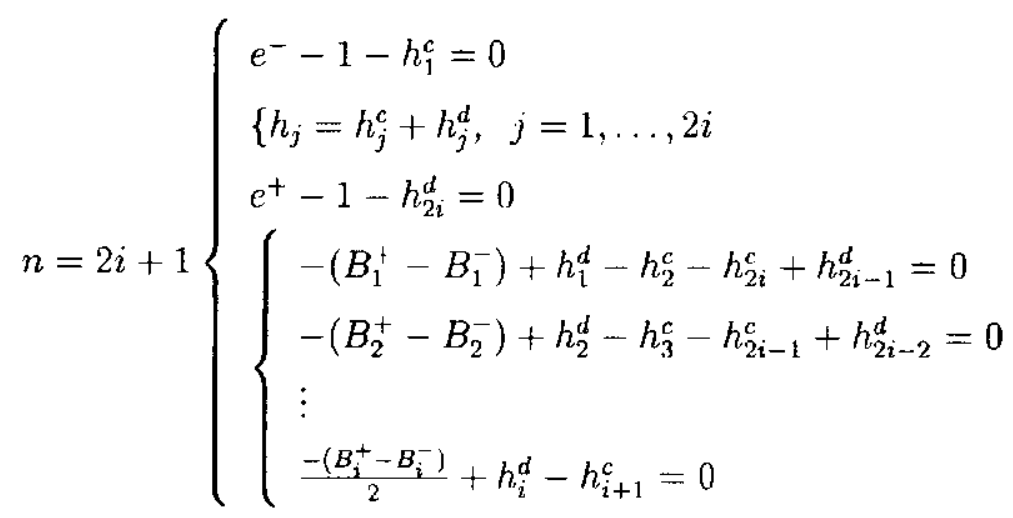




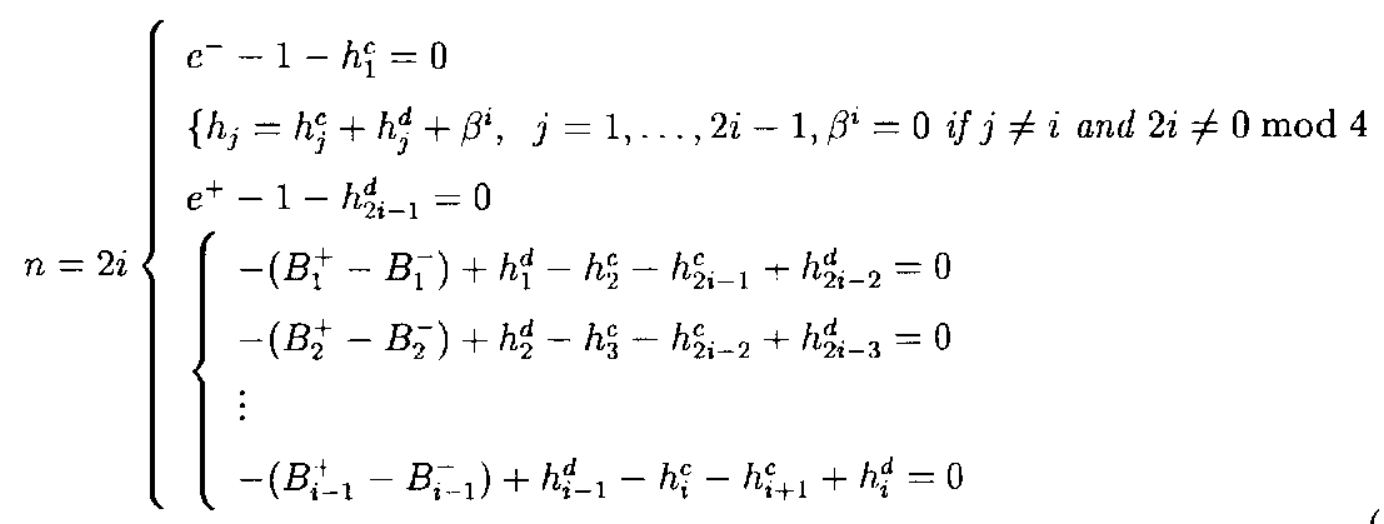

Neste capítulo trabalharemos com um caso particular da Proposição 4.1.1 tomando $B_{j}^{-}=B_{j}^{+}$. Logo o sistema (4.1) se reduz a (4.3) e o sistema (4.2) se reduz a (4.34) no caso $n=0 \bmod 4$ on a (4.61) no caso $n=2 \bmod 4$. Pela Proposiçăo 4.1.1, os sistemas (4.3), (4.34) e (4.61) têm uma solução inteira não negativa $\left(h_{1}^{r} h_{1}^{d}, \ldots, h_{n-1}^{c} h_{n-1}^{d}\right)$ se, e somente se, as desigualdades de Poincaré-Hopf (2.21), (2.22) e (2.23) são satisfeitas.

Subseqüentemente mostramos que (4.3) (resp., (4.61)) tem soluções inteiras não negativas se, e somente se, existem vetores de númcros de Betti inteiros não negativos que satisfazem as desigualdades de Morse (4.5) (resp., (4.64)). O resultado envolvendo (4.34) e (4.38), correspondendo ao caso $n=0 \bmod 4$, acrescenta a hipótese adicional que os dados de índice pré-fixados $\left(h_{0}, \ldots, h_{n}\right)$ satisfazem a condição que o número $\sum_{j=0}^{n}(-1)^{j+1} h_{j}$ é par.

\subsubsection{Caso $n$ ímpar}

Suponha que existarn inteiros não negativos $\left(h_{0}, h_{1}, \ldots, h_{2 i+1}, h_{1}^{c}, h_{1}^{d}, \ldots, h_{2 i}^{c}, h_{2 i}^{d}\right)$, onde $i \geq 1$, que satisfazem o sistema linear

$$
\left\{\begin{aligned}
h_{1}^{c} & =h_{0}-1 \\
h_{j}^{c}+h_{j}^{d} & =h_{j}, \quad \text { para } j=1, \ldots, 2 i \\
h_{2 i}^{d} & =h_{2 i+1}-1 \\
h_{j}^{d}-h_{j+1}^{c}+h_{2 i-j}^{d}-h_{2 i-j+1}^{c} & =0, \quad \text { para } j=1, \ldots, i-1 \\
h_{i}^{d}-h_{i+1}^{c} & =0 .
\end{aligned}\right.
$$


Considere os inteiros não negativos fixados $\left(h_{0}, h_{1}, \ldots, h_{2 i+1}\right)$ que formam parte de uma solução de (4.3). Então, para estes $\left(h_{0}, h_{1}, \ldots, h_{2 i+1}\right)$ fixados, existem inteiros não negativos $\left(h_{1}^{c}, h_{1}^{d}, h_{2}^{c}, h_{2}^{d}, \ldots, h_{2 i}^{c}, h_{2 i}^{d}\right)$ que resolvem (4.3) e portanto são também solução do sistema equivalente abaixo, obtido multiplicando as equações ímpares em (4.3) por -1 :

$$
\left\{\begin{aligned}
-h_{1}^{c} & =-\left(h_{0}-1\right) \\
(-1)^{j+1}\left(h_{j}^{c}+h_{j}^{d}\right) & =(-1)^{j+1} h_{j}, \quad \text { para } j=1, \ldots, 2 i \\
h_{2 i}^{d} & =h_{2 i+1}-1 \\
(-1)^{j}\left(h_{j}^{d}-h_{j+1}^{c}+h_{2 i-j}^{d}-h_{2 i-j+1}^{c}\right) & =0, \\
(-1)^{i}\left(h_{i}^{d}-h_{i+1}^{c}\right) & =0 .
\end{aligned}\right.
$$

Pode-se mostrar, como no Capítulo 3, que o sistema (4.4) constitui um problema de fluxo de rede. A matriz de coeficientes de (4.4) é a matriz de incidência nó-arco de um digrafo. As variáveis representam fluxo ao longo dos arcos e cada equação em (4.4) garante o equilíbrio de fluxo no nó correspondente. A rede contém uma cadeia de $i-1$ ciclos de comprimento quatro. Os arcos no $j$-ésimo ciclo estão associados às variáveis $h_{j+1}^{d}, h_{2 i \cdot j}^{c}, h_{2 i-j}^{d}$ e $h_{j+1}^{c}$, e a orientação dos dois primeiros arcos é oposta à orientação dos dois últimos, com respeito a uma orientação arbitrária do ciclo. Os nós no $j$-ésimo ciclo estão associados às equaçóes $j+2,2 i+1-j, 2 i+2+j$ e $2 i+3+j$, de (4.4). Assim os nós associados à $(2 i+2+j)$-ésima equação do (4.4) constitui a interseção de ciclos $j-1$ e $j$. A seqüência de arcos associadas com $\left(h_{1}^{c}, h_{1}^{d}, h_{2 i}^{c}, h_{2 i}^{d}\right)$, as variáveis que ainda nāo foram consideradas, formam um caminho não orientado que é adjacente ao primeiro ciclo. A interseção deste caminho e o primeiro ciclo é o nó associado à equação $2 i+3$ de (4.4). Arcos correspondendo às variáveis de fluxo $\left(h_{1}^{c}, h_{1}^{d}, h_{2}^{c}, h_{2}^{d}, \ldots, h_{2 i}^{c}, h_{2 i}^{d}\right)$, nesta ordem, formam um caminho Euleriano não orientado percorrendo cada arco do digrafo. Este caminho tem uma forma de zig-zag na imersão planar do digrafo exemplificada na Figura 4.1 para o caso $i=3$. No interior de cada nó escrevemos o elemento do lado direito da equação de equilíbrio de fluxo correspondente.

Proposição 4.1.2 Se, para um dado conjunto de inteiros $\left(h_{0}, h_{1}, \ldots, h_{2 i}, h_{2 i+1}\right)$ o sistema (4.4) tem uma solução inteira não negativa, então existem inteiros não negativos 


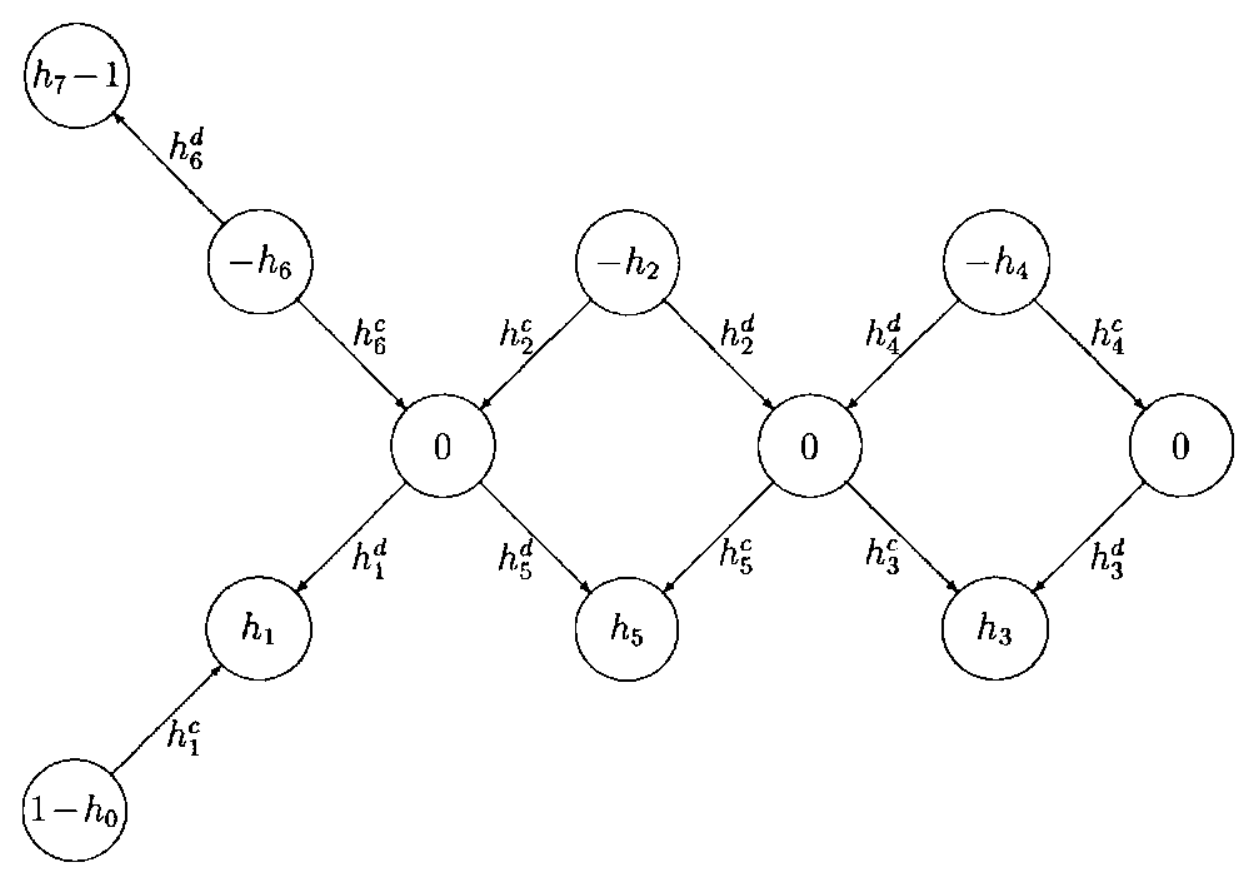

Figura 4.1: Examplo de rede, para $i=3$.

$\left(\gamma_{0}, \gamma_{1}, \ldots, \gamma_{2 i}, \gamma_{2 i+1}\right)$ satisfazendo

$$
\sum_{j=0}^{2 i+1-k}(-1)^{j+1} \gamma_{j}\left\{\begin{array}{l}
=\sum_{j-0}^{2 i+1}(-1)^{j+1} h_{j}, \text { se } k=0 \\
\geq \sum_{j=0}^{2 i+1 k}(-1)^{j+1} h_{j}, \quad \text { se } 1 \leq k \leq 2 i+1, k \text { impar } \\
\leq \sum_{j=0}^{2 i+1-k}(-1)^{j+1} h_{j}, \quad \text { se } 1 \leq k \leq 2 i+1, k \text { par }
\end{array}\right.
$$

$e$

$$
\begin{aligned}
& \gamma_{0}=\gamma_{2 i+1}=1 \\
& \gamma_{j}=\gamma_{2 i+1-j}, \quad \text { para } 1 \leq j \leq i
\end{aligned}
$$

Demonstração: Seja $h^{c d}=\left(h_{1}^{c}, h_{1}^{d}, h_{2}^{c}, h_{2}^{d}, \ldots, h_{2 i}^{c}, h_{2 i}^{d}\right)$ uma solução inteira não negativa de (4.4). Assuma, sem perda de generalidade, que $h^{\text {cd }}$ satisfaz as equaçōes

$$
h_{j}^{c} h_{2 i+1-j}^{d}=0, \quad \text { para } j=2, \ldots, i
$$


já que, dado um fluxo arbitrário $h^{c d}$ satisfazendo 4.4), podemos reduzir a zero pelo menos um dos fluxos em $(4.8)$ enviando ao $(j-1)$-ésimo ciclo uma circulação de valor $\min \left\{h_{j}^{c}, h_{2 i+1-j}^{d}\right\}$ na direção oposta aquela do arco associado with $h_{j}^{c}$. A circulação inteira assim construída é uma solução da versão homogênea do sistema (4.4), cuja soma ao fluxo (uma solução do sistema (4.4)) resulta em um outro fluxo inteiro não negativo satisfazendo (4.4) e (4.8).

Afirmamos que o vetor $\gamma$ definido por

$$
\begin{aligned}
& \gamma_{0}=\gamma_{2 i+1}=1 \\
& \gamma_{j}= \begin{cases}h_{j}^{d}-h_{j+1}^{c}, & \text { se } 1<j<i \\
h_{i}^{d}, & \text { se } j=i \\
h_{i+1}^{c}, & \text { se } j=i+1 \\
-h_{j-1}^{d}+h_{j}^{c}, & \text { se } i+2 \leq j \leq 2 i\end{cases}
\end{aligned}
$$

é uma solução intcira não negativa de (4.5), (4.6) e (4.7).

A integralidade de $\gamma$ segue facilmente da integralidade de $h^{c d}$. Alćm disso, as equações $2 i+2+j$ de (4.4) implicam em

a) Se $1 \leq j<i$

$$
\begin{aligned}
(-1)^{j}\left(h_{j}^{d}-h_{j+1}^{c}+h_{2 i-j}^{d}-h_{2 i-j+1}^{c}\right) & = \\
(-1)^{j}\left(\gamma_{j}-\gamma_{2 i+1-j}\right) & =0 .
\end{aligned}
$$

b) Se $j=i$

$$
\begin{aligned}
& (-1)^{i}\left(h_{i}^{d}-h_{i+1}^{c}\right)= \\
& (-1)^{i}\left(\gamma_{i}-\gamma_{i+1}\right)=0 .
\end{aligned}
$$

As equaçōes (4.10) e (4.11), e a definição de $\gamma_{0}$ e $\gamma_{2 i+1}$ dadas em (4.9) implicam que $\gamma$ definido acima satifaz as condiçōes de fronteira e simetria (4.6)-4.7).

Dado que (4.7) está estabelecida, a não negatividade de $\gamma$ é estabelecida se mostrarmos que $\gamma_{j}$ ou $\gamma_{2 i+1-j}$, para $0 \leq j \leq i$, é não negativo. Isto é trivialmente verdadeiro para $j=0$ e $i$. Considere $1 \leq j \leq i-1$. Por definição e (4.7),

$$
\gamma_{j}=h_{j}^{d}-h_{j+1}^{c}=-h_{2 i-j}^{d}+h_{2 i+1-j}^{c}=\gamma_{2 i+1-j} .
$$


De (4.8) temos que $h_{j+1}^{c} h_{2 i-j}^{d}=0$. Se $h_{j+1}^{c}=0$, então $\gamma_{2 i+1-j}=\gamma_{j}=h_{j}^{d}-h_{j+1}^{c}=h_{j}^{d} \geq 0$. Se $h_{2 i-j}^{d}=0$, então $\gamma_{j}=\gamma_{2 i+1-j}=-h_{2 i-j}^{d}+h_{2 i+1-j}^{c}=h_{2 i+1-j}^{c} \geq 0$. Portanto o vetor $\gamma$ definido acima é nāo negativo. Mostraremos a seguir que o vetor também satisfaz (4.5).

Somando as equações 1 até $2 i+2$ de (4.4) obtemos

$$
\begin{aligned}
-h_{1}^{c}+\sum_{j=1}^{2 i}(-1)^{j+1}\left(h_{j}^{c}+h_{j}^{d}\right)+h_{2 i}^{d} & = \\
-h_{1}^{c}+h_{1}^{c}+h_{1}^{d}+\sum_{j=2}^{2 i-1}(-1)^{j+1}\left(h_{j}^{c}+h_{j}^{d}\right)-h_{2 i}^{c}-h_{2 i}^{d}+h_{2 i}^{d} & = \\
h_{1}^{d}+\sum_{j=2}^{2 i-1}(-1)^{j+1}\left(h_{j}^{c}+h_{j}^{d}\right)-h_{2 i}^{c} & = \\
\sum_{j=1}^{2 i-1}(-1)^{j+1} h_{j}^{d}+\sum_{j=2}^{2 i}(-1)^{j+1} h_{j}^{c} & = \\
\sum_{j-1}^{2 i-1}(-1)^{j+1} h_{j}^{d}+\sum_{j=1}^{2 i-1}(-1)^{j} h_{j+1}^{c} & = \\
\sum_{j=1}^{2 i-1}(-1)^{j+1}\left(h_{j}^{d}-h_{j+1}^{c}\right) & =-\left(h_{0}-1\right)+\sum_{j=1}^{2 i}(-1)^{j+1} h_{j}+h_{2 i+1}-1 \\
& =\sum_{j=0}^{2 i+1}(-1)^{j+1} h_{j} .
\end{aligned}
$$

A soma alternada de $\gamma$ 's, de acordo com (4.9), resulta $\mathrm{cm}$

$$
\begin{aligned}
\sum_{j=0}^{2 i+1}(-1)^{j+1} \gamma_{j}= & -1+\sum_{j=1}^{i-1}(-1)^{j+1}\left(h_{j}^{d}-h_{j+1}^{c}\right)+(-1)^{i+1} h_{i}^{d}+(-1)^{i+2} h_{i+1}^{c}+ \\
& \sum_{j=i+2}^{2 i}(-1)^{j+1}\left(-h_{j-1}^{d}+h_{j}^{c}\right)+1 \\
= & \sum_{j=1}^{i}(-1)^{j+1}\left(h_{j}^{d}-h_{j+1}^{c}\right)+\sum_{j=i+1}^{2 i-1}(-1)^{j}\left(-h_{j}^{d}+h_{j+1}^{c}\right) \\
= & \sum_{j=1}^{2 i-1}(-1)^{j+1}\left(h_{j}^{d}-h_{j+1}^{c}\right) .
\end{aligned}
$$

As equaçōes (4.12) e (4.13) implicam que

$$
\sum_{j=0}^{2 i+1}(-1)^{j+1} \gamma_{j}=\sum_{j=0}^{2 i+1}(-1)^{j+1} h_{j},
$$

isto é, $\gamma$ satisfaz a primeira equação em (4.5). 
Agora considere a soma das equações 1 até $2 i+2-\ell$ de (4.4), onde $1 \leq \ell \leq 2 i$ :

$$
\begin{aligned}
-h_{1}^{c}+\sum_{j=1}^{2 i+1-\ell}(-1)^{j+1}\left(h_{j}^{c}+h_{j}^{d}\right) & = \\
h_{1}^{d}+\sum_{j=2}^{2 i+1-\ell}(-1)^{j+1}\left(h_{j}^{c}+h_{j}^{d}\right) & = \\
\sum_{j=1}^{2 i-\ell}(-1)^{j+1} h_{j}^{d}+\sum_{j=2}^{2 i+1-\ell}(-1)^{j+1} h_{j}^{c}+(-1)^{2 i+2-\ell} h_{2 i+1-\ell}^{d} & = \\
\sum_{j=1}^{2 i-\ell}(-1)^{j+1}\left(h_{j}^{d}-h_{j+1}^{c}\right)+(-1)^{2 i+2-\ell} h_{2 i+1-\ell}^{d} & =-\left(h_{0}-1\right)+\sum_{j=1}^{2 i+1-\ell}(-1)^{j+1} h_{j} .
\end{aligned}
$$

A última igualdade implica que

$$
-1+\sum_{j=1}^{2 i-\ell}(-1)^{j+1}\left(h_{j}^{d}-h_{j+1}^{c}\right)+(-1)^{2 i+2-\ell} h_{2 i+1-\ell}^{d}=\sum_{j=0}^{2 i+1-\ell}(-1)^{j+1} h_{j} .
$$

Consideramos três casos, quando calculamos a soma alternada parcial das primeiras $2 i+2-\ell$ componentes de $\gamma$ :

a) $1 \leq \ell \leq i$

$$
\begin{aligned}
\sum_{j=0}^{2 i+1-\ell}(-1)^{j+1} \gamma_{j}= & -1+\sum_{j=1}^{i-1}(-1)^{j+1}\left(h_{j}^{d}-h_{j+1}^{c}\right)+(-1)^{i+1} h_{i}^{d}+(-1)^{i+2} h_{i+1}^{c}+ \\
& \sum_{j=i+2}^{2 i+1-\ell}(-1)^{j+1}\left(-h_{j-1}^{d}+h_{j}^{c}\right) \\
= & -1+\sum_{j=1}^{i}(-1)^{j+1}\left(h_{j}^{d}-h_{j+1}^{c}\right)+\sum_{j=i+1}^{2 i-\ell}(-1)^{j}\left(-h_{j}^{d}+h_{j+1}^{c}\right) \\
= & -1+\sum_{j=1}^{2 i-\ell}(-1)^{j+1}\left(h_{j}^{d}-h_{j+1}^{c}\right) .
\end{aligned}
$$

Substituindo (4.16) em (4.15) obtemos

$$
\sum_{j=0}^{2 i+1-\ell}(-1)^{j+1} \gamma_{j}+(-1)^{2 i+2-\ell} h_{2 i+1-\ell}^{d}=\sum_{j=0}^{2 i+1-\ell}(-1)^{j+1} h_{j} .
$$

Taking into account the fact that $h_{j}^{d} \geq 0$ para todo $j=1, \ldots, 2 i$, a equação (4.17) 
implica

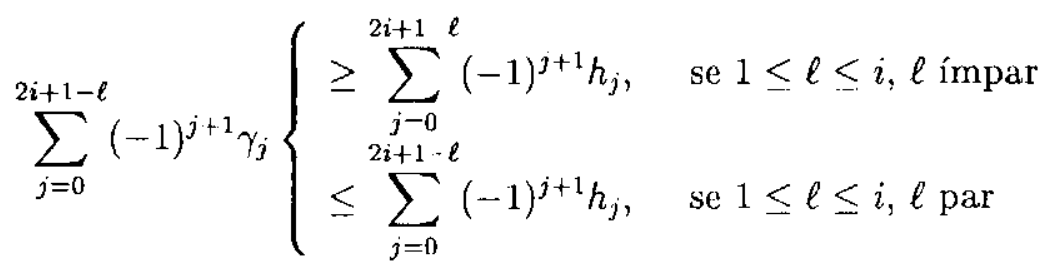

o que significa que $\gamma$ definido em (4.9) satisfaz as desigualdades em (4.5), para $1 \leq \ell \leq i$.

b) $\ell=i+1$

$$
\begin{aligned}
\sum_{j=0}^{2 i+1-\ell}(-1)^{j+1} \gamma_{j} & =-1+\sum_{j=1}^{i-1}(-1)^{j+1}\left(h_{j}^{d}-h_{j+1}^{c}\right)+(-1)^{i+1} h_{i}^{d} \\
& =-1+\sum_{j=1}^{2 i-\ell}(-1)^{j+1}\left(h_{j}^{d}-h_{j+1}^{c}\right)+(-1)^{2 i+2-\ell} h_{2 i+1-\ell}^{d} .
\end{aligned}
$$

De (4.19) e (4.15) concluímos que, para $\ell=i+1$,

$$
\sum_{j=0}^{i}(-1)^{j+1} \gamma_{j}=\sum_{j=0}^{i}(-1)^{j+1} h_{j}
$$

e assim $\gamma$ também satisfaz (1.5) para $\ell=i+1$.

c) $i+2 \leq \ell \leq 2 i$

$$
\begin{aligned}
\sum_{j=0}^{2 i+1-\ell}(-1)^{j+1} \gamma_{j} & =-1+\sum_{j=1}^{2 i+1-\ell}(-1)^{j+1}\left(h_{j}^{d}-h_{j+1}^{c}\right) \\
& =-1+\sum_{j=1}^{2 i-\ell}(-1)^{j+1}\left(h_{j}^{d}-h_{j+1}^{c}\right)+(-1)^{2 i+2-\ell}\left(h_{2 i+1-\ell}^{d}-h_{2 i+(A-2)}^{c}\right)
\end{aligned}
$$

Usando (4.21) e (4.15) obtemos

$$
\sum_{j=0}^{2 i+1-\ell}(-1)^{j+1} \gamma_{j}+(-1)^{2 i+2-\ell} h_{2 i+2-\ell}^{c}=\sum_{j=0}^{2 i+1-\ell}(-1)^{j+1} h_{j},
$$

que implica que (4.18), dando a não negatividade de $h_{j}^{c}$, para todo $j$.

A primeira equação em $(4.4),-h_{1}^{c}=-\left(h_{0}-1\right)$ e o fato que $h_{1}^{c} \geq 0$ implicam que $h_{0} \geq 1=\gamma_{0}$, assim a última desigualdade $\mathrm{em} \mathrm{(4.5)} \mathrm{é} \mathrm{também} \mathrm{satisfeita.}$

Assim estabelecemos que o $\gamma$ definido $\mathrm{cm}$ (4.9) satisfaz as restrições em (4.5).

A seguinte proposição estabelece a recíproca da Proposição 4.1.2, i.e., se (4.5)-(4.7) tem uma solução inteira não negativa então (4.3) também tem. 
Proposição 4.1.3 Se, para um dado conjunto de inteiros não negativos $\left(h_{0}, h_{1}, \ldots, h_{2 i}\right.$, $\left.h_{2 i+1}\right)$, existe um inteiro não negativo $\gamma=\left(\gamma_{0}, \gamma_{1}, \ldots, \gamma_{2 i}, \gamma_{2 i+1}\right)$ que satisfaz (4.5) (4.7), então o sistema (4.3) tem uma solução inteira não negativa.

Demonstração: Assumimos scm perda de generalidade que a solução inteira não negativa $\gamma$ de (4.5) (4.7) satura a desigualdade correspondente a $k=i+1$ em (4.5),

$$
\sum_{j=0}^{i}(-1)^{j+1} \gamma_{j}=\sum_{j=0}^{i}(-1)^{j+1} h_{j}
$$

já que, se fixamos em seus valores correntes todos $\gamma^{\prime}$ 's exceto $\gamma_{i}$ e $\gamma_{i+1}$, que deixamos livres para variar, então todas as desigualdades em (4.5-4.7) são satisfeitas contanto que as seguintes condições se mantenham:

$$
\begin{aligned}
\gamma_{i} & =\gamma_{i+1} \\
(-1)^{i+1} \sum_{j=0}^{i}(-1)^{j+1} \gamma_{j} & \leq(-1)^{i+1} \sum_{j=0}^{i}(-1)^{j+1} h_{j} \\
\gamma_{i}, \gamma_{i+1} & \geq 0 .
\end{aligned}
$$

Ista é devido ao fato que todas as desigualdades lineares em (4.5) exceto a acima contêm a diferença $\pm\left(\gamma_{i}-\gamma_{i+1}\right)$ ou não contêm $\gamma_{i}$ nem $\gamma_{i+1}$. Segue que $0 \leq \gamma_{i}=\gamma_{i+1} \leq(-1)^{i+1}\left(\sum_{j=0}^{i}(-1)^{j+1} h_{j}-\sum_{j=0}^{i-1}(-1)^{j+1} \gamma_{j}\right)=h_{i}+(\cdots 1)^{i+1} \sum_{j=0}^{i-1}(-1)^{j+1}\left(h_{j}-\gamma_{j}\right)$.

O intervalo $\left[0, h_{i}+(-1)^{i+1} \sum_{j=0}^{i-1}(-1)^{j+1}\left(h_{j}-\gamma_{j}\right)\right]$ é não vazio (contém o valor corrente de $\gamma_{i}$ ) e tem extremos inteiros, já que $\gamma$ é inteiro e não negativo por suposição. Assim podemos assumir que $\gamma_{i}$ (e, portanto, $\gamma_{i+1}$ ) está no extremo direito do intervalo acima.

Agora defina o vetor $h^{\text {cd }}$ de acordo com

$$
\begin{array}{rlrl}
h_{2 i+1-\ell}^{d} & =(-1)^{\ell} \sum_{j=0}^{2 i+1 \cdot \ell}(-1)^{j+1}\left(h_{j}-\gamma_{j}\right), & \text { para } 1 \leq \ell \leq i \\
h_{2 i+2-\ell}^{c} & =(-1)^{\ell} \sum_{j=0}^{2 i+1-\ell}(-1)^{j+1}\left(h_{j}-\gamma_{j}\right), & \text { para } & i+2 \leq \ell \leq 2 i+1 \\
h_{\ell}^{d} & =\gamma_{\ell}+h_{\ell+1}^{c}, & & \text { para } 1 \leq \ell \leq i-1 \\
h_{\ell}^{c} & =\gamma_{\ell}+h_{\ell-1}^{d}, & & \text { para } i+2 \leq \ell \leq 2 i \\
h_{i}^{d} & =\gamma_{i} & \\
h_{i+1}^{c} & =\gamma_{i+1} . &
\end{array}
$$


Afirmamos que o vetor $h^{c d}$ assim definido é uma solução inteira não negativa de (4.3). A integralidade segue facilmente da integralidade de $\gamma$ e $h$. Reescrevendo (4.5) como

$$
(-1)^{k} \sum_{j=0}^{2 i+1-k}(-1)^{j+1} \gamma_{j}\left\{\begin{array}{l}
=\sum_{j=0}^{2 i+1}(-1)^{j+1} h_{j}, \quad \text { se } k=0 \\
\leq(-1)^{k} \sum_{j-0}^{2 i+1-k}(-1)^{j+1} h_{j}, \quad \text { se } 1 \leq k \leq 2 i+1
\end{array}\right.
$$

concluímos que as componentes de $h^{\text {cd }}$ definidas em (4.27) e (4.28) são não negativas. Este fato, por outro lado, juntamente com as hipóteses $h \geq 0$ e $\gamma \geq 0$, implicam que as componentes definidas em (4.29)-(4.32) são também não negativas.

Agora devemos verificar que $h^{\text {cd }}$ satisfaz as restrições em (4.3). As equações (4.28) para $\ell=2 i+1$ e (4.6) implicam

$$
h_{1}^{\epsilon}=(-1)^{2 \mathrm{n}+1}(-1)^{1}\left(h_{0}-\gamma_{0}\right)=h_{0}-1,
$$

$\operatorname{assim} h^{\text {cd }}$ satisfaz a primeira equação em (4.3). As equações (4.27) para $\ell=1$, (4.6) e a primeira equação de (4.5) implicam

$$
\begin{aligned}
h_{2 i}^{d} & =(-1)^{1} \sum_{j=0}^{2 i}(-1)^{j+1}\left(h_{j}-\gamma_{j}\right) \\
& =-\left(\sum_{j=0}^{2 i+1}(-1)^{j+1}\left(h_{j}-\gamma_{j}\right)-\left(h_{2 i+1}-\gamma_{2 i+1}\right)\right) \\
& =h_{2 i+1}-\gamma_{2 i+1} \\
& =h_{2 i+1}-1,
\end{aligned}
$$

que implica a $(2 i+2)$-ésima equação de (4.3).

As últimas $i$ equaçōes de (4.3),

$$
\begin{array}{rlr}
h_{j}^{d}-h_{j+1}^{c}+h_{2 i-j}^{d}-h_{2 i+1-j}^{c} & =\left(\gamma_{j}+h_{j+1}^{c}\right)-h_{j+1}^{c}+h_{2 i-j}^{d}-\left(\gamma_{2 i+1-j}+h_{2 i, j}^{d}\right) \\
& =\gamma_{j}-\gamma_{2 i+1-j}=0, & \\
h_{i}^{d}-h_{i+1}^{c} & =\gamma_{i}-\gamma_{i+1}=0, &
\end{array}
$$

sāo válidas usando (4.7).

Seja $i+2 \leq \ell \leq 2 i$. Somando as equações adequadas cm (4.28) obtemos

$$
\begin{aligned}
h_{2 i+2-\ell}^{c}+h_{2 i+1-\ell}^{c} & =(-1)^{\ell}\left(\sum_{j=0}^{2 i+1-\ell}(-1)^{j+1}\left(h_{j}-\gamma_{j}\right)-\sum_{j=0}^{2 i-\ell}(-1)^{j+1}\left(h_{j}-\gamma_{j}\right)\right) \\
& =(-1)^{\ell}(-1)^{2 i+2-\ell}\left(h_{2 i+1-\ell}-\gamma_{2 i+1-\ell}\right) \\
& =h_{2 i+1-\ell}-\gamma_{2 i+1-\ell},
\end{aligned}
$$


que implica, usando (4.29), a $(2 i+2-\ell)$-ésima equação de (1.3):

$$
\gamma_{2 i+1-\ell}+h_{2 i+2-\ell}^{c}+h_{2 i+1-\ell}^{c}=h_{2 i+1-\ell}^{d}+h_{2 i+1-\ell}^{c}=h_{2 i+1-\ell} .
$$

Assim, as equações $(1+j)$ de $(4.3)$, para $1 \leq j \leq i-1$, são satisfeitas por $h^{\text {rd }}$.

A $(1+i)$-ésima equação de (4.3) segue de (4.28), (4.31) e (4.26):

$$
\begin{aligned}
h_{i}^{c}+h_{i}^{d} & =(-1)^{i+2} \sum_{j=0}^{i-1}(-1)^{j+1}\left(h_{j}-\gamma_{j}\right)+\gamma_{i} \\
& =(-1)^{i+2} \sum_{j=0}^{i-1}(-1)^{j+1}\left(h_{j}-\gamma_{j}\right)+h_{i}+(-1)^{i+1} \sum_{j=0}^{i-1}(-1)^{j+1}\left(h_{j}-\gamma_{j}\right) \\
& =h_{i},
\end{aligned}
$$

e a $(1+(i+1))$-ésima equação de (4.3) segue de (4.27), (4.32) e (4.26):

$$
\begin{aligned}
h_{i+1}^{c}+h_{i+1}^{d} & =\gamma_{i+1}+(-1)^{i} \sum_{j=0}^{i+1}(-1)^{j+1}\left(h_{j}-\gamma_{j}\right) \\
& =h_{i}+(-1)^{i+1} \sum_{j=0}^{i \cdots 1}(-1)^{j+1}\left(h_{j}-\gamma_{j}\right)+(-1)^{i} \sum_{j=0}^{i+1}(-1)^{j+1}\left(h_{j}-\gamma_{j}\right) \\
& =h_{i}+(-1)^{i}\left((-1)^{i+1}\left(h_{i}-\gamma_{i}\right)+(-1)^{i+2}\left(h_{i+1}-\gamma_{i+1}\right)\right) \\
& =h_{i}-\left(h_{i}-\gamma_{i}\right)+\left(h_{i+1}-\gamma_{i+1}\right) \\
& =h_{i+1} .
\end{aligned}
$$

Finalmente, mostremos que $h^{c d}$ satisfaz as equaçōes restantes de (4.3): equações $(i+$ $2+j$ ), para $1 \leq j \leq i-1$. Seja $1 \leq \ell<i-1$. Usando (4.27) temos

$$
\begin{aligned}
h_{2 i+1-\ell}^{d}+h_{2 i-\ell}^{d} & =(-1)^{\ell} \sum_{j=0}^{2 i+1-\ell}\left({ }_{1}\right)^{j+1}\left(h_{j}-\gamma_{j}\right)+(-1)^{e l l+1} \sum_{j=0}^{2 i-\ell}(1)^{j+1}\left(h_{j}-\gamma_{j}\right) \\
& =(-1)^{\ell}(-1)^{2 i+2-\ell}\left(h_{2 i+1-\ell}-\gamma_{2 i+1-\ell}\right) .
\end{aligned}
$$

Portanto, considerando a definição (4.30)

$$
h_{2 i+1-\ell}^{d}+\gamma_{2 i+1-\ell}+h_{2 i-\ell}^{d}=h_{2 i+1-\ell}^{d}+h_{2 i+1-\ell}^{\mathrm{c}}=h_{2 i+1-\ell} .
$$




\subsubsection{Caso $\mathrm{n}=0 \bmod 4$}

Assuma $n=2 i$, onde $i \geq 2$ ć par. Suponha que existam inteiros não negativos $\left(h_{0}, h_{1}, \ldots, h_{2 i+1}, h_{1}^{c}, h_{1}^{d}, \ldots, h_{2 i}^{c}, h_{2 i}^{d}\right)$ que satisfazem o sistema linear

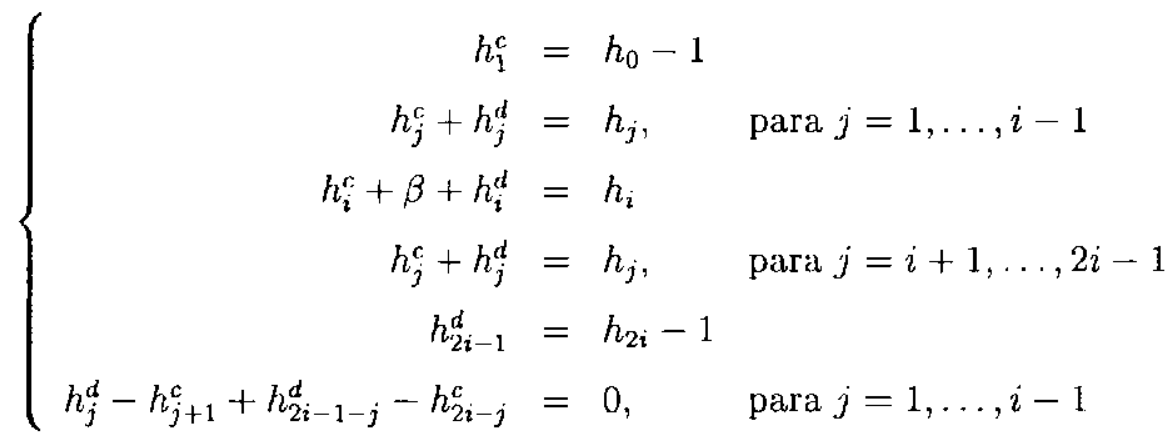

Fixc os valores inteiros não negativos $\left(h_{0}, h_{1}, \ldots, h_{2 i}\right)$ tais que (4.34) tem uma solução inteira não negativa $\left(h_{1}^{c}, h_{1}^{d}, h_{2}^{c}, h_{2}^{d}, \ldots, h_{2 i-1}^{c}, h_{2 i-1}^{d}\right)$. Então este último vetor satisfaz (4.35) abaixo, equivalente a (4.34), obtido multiplicando-se por -1 as equações ímpares até a $i+1$-ésima e as equações pares daí por diante:

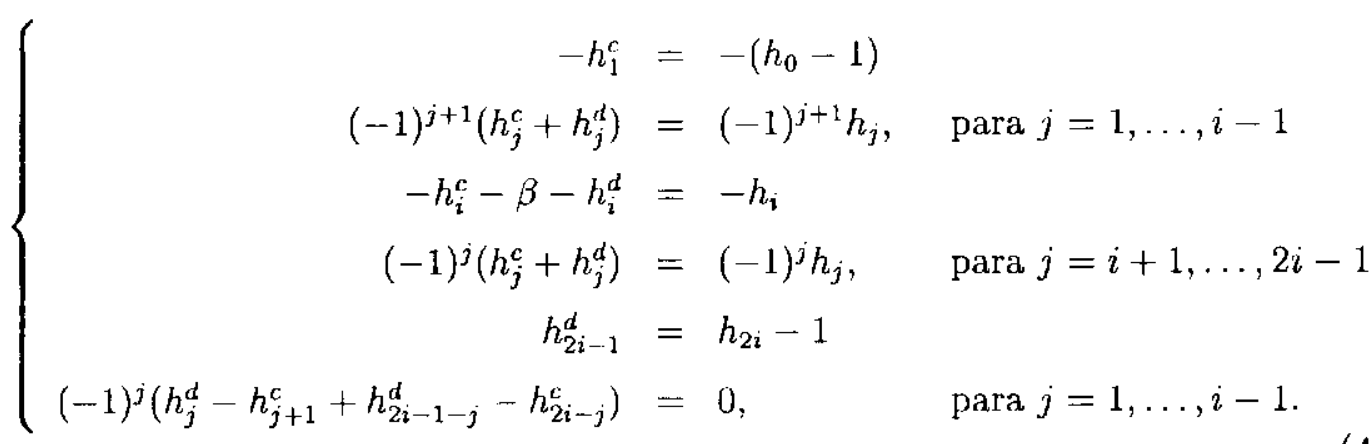

O sistema (4.35) pode ser decomposto, veja o Capítulo 3, em dois sistemas independentes: (4.36) e (4.37). O sistema (4.36) é um problema de fluxo em rede definido sobre um digrafo cuja matriz de incidência é a matriz de coeficientes de (4.4) para $n=2 i-1$. Assim para o caso $i=4$ o digrafo tem precisamente a estrutura descrita na Figura 4.1, mas naturalmente com diferentes rótulos sobre arcos e nós. No caso geral, o digrafo com matriz de incidência dado pela matriz de cocficientes de (4.36) tem $i-2$ ciclos de comprimento quatro mais um caminho não orientado também de comprimento quatro. O $j$-ésimo ciclo contém arcos associados às variávcis $h_{j+1}^{d}, h_{2 i-1-j}^{c}, h_{2 i-1-j}^{d}$ e $h_{j+1}^{c}$, e a orientação dos dois primeiros arcos é oposta à orientação dos dois últimos, com respeito 
a uma orientação arbitrária do ciclo. Nós do $j$-ésimo ciclo estão associados às equações $j+2,2 i+2+j, 2 i-j$ e $2 i+1+j$.

$$
\begin{aligned}
& \left\{\begin{array}{rlrl}
-h_{1}^{c} & =-\left(h_{0}-1\right) & \\
(-1)^{j+1}\left(h_{j}^{c}+h_{j}^{d}\right) & =(-1)^{j+1} h_{j}, \quad & & \text { para } j=1, \ldots, i-1 \\
(-1)^{j}\left(h_{j}^{c}+h_{j}^{d}\right) & =(-1)^{j} h_{j}, & & \text { para } j=i+1, \ldots, 2 i-1 \\
h_{2 i-1}^{d} & =h_{2 i}-1 & \\
(-1)^{j}\left(h_{j}^{d}-h_{j+1}^{c}+h_{2 i-1-j}^{d}-h_{2 i-j}^{c}\right) & =0, & \\
-h_{i-1}^{d}+h_{i+1}^{c} & =-\sum_{j=0}^{i-1}(-1)^{j+1} h_{j}-\sum_{j=i+1}^{2 i}(-1)^{j} h_{j}
\end{array}\right. \\
& \left\{\begin{aligned}
-h_{i}^{c}-\beta-h_{i}^{d} & =-h_{i} \\
h_{i}^{c}-h_{i}^{d} & =\sum_{j=0}^{i-1}(-1)^{j+1} h_{j}+\sum_{j=i+1}^{2 i}(-1)^{j} h_{j}
\end{aligned}\right.
\end{aligned}
$$

Proposição 4.1.4 Se, para um dado conjunto de inteiros não negativos $\left(h_{0}, h_{1}, \ldots, h_{2 i}\right)$ o sistema (4.35) tem uma solução inteira não negativa, então existem inteiros não negativos $\left(\gamma_{0}, \gamma_{1}, \ldots, \gamma_{2 i}\right)$ satisfazendo

$$
\sum_{j=0}^{2 i-k}(-1)^{j+1} \gamma_{j} \begin{cases}=\sum_{j=0}^{2 i}(-1)^{j+1} h_{j}, & \text { se } k=0 \\ \leq \sum_{j=0}^{2 i-k}(-1)^{j+1} h_{j}, & \text { se } 1 \leq k \leq 2 i, k \text { impar } \\ \geq \sum_{j=0}^{2 i-k}(-1)^{j+1} h_{j}, & \text { se } 1 \leq k \leq 2 i, k \text { par }\end{cases}
$$

$e$

$$
\begin{aligned}
& \gamma_{0}=\gamma_{2 i}=1 \\
& \gamma_{j}=\gamma_{2 i-j}, \quad \text { para } 1 \leq j \leq i-1 .
\end{aligned}
$$

O vetor $\gamma$ satisfaz também a condição $\gamma_{i}$ par se, e somente se, $\sum_{j=0}^{2 i}(-1)^{j} h_{j}$ é par.

Demonstração: Se $\gamma$ satisfaz (4.38) e (4.40) então

$$
\begin{aligned}
\sum_{j=0}^{2 i}(-1)^{j+1} \gamma_{j} & = \\
2 \sum_{j=0}^{i-1}(-1)^{j+1} \gamma_{j}+(-1)^{i+1} \gamma_{i} & =\sum_{j=0}^{2 i}(-1)^{j+1} h_{j} .
\end{aligned}
$$


Portanto se $\gamma_{i}=\sum_{j=0}^{2 i}(-1)^{j} h_{j}-2 \sum_{j=0}^{i-1}(-1)^{j+1} \gamma_{j}$ é par, cntão $\sum_{j=0}^{2 i}(-1)^{j} h_{j}$ é também par.

Por outro lado, se $\sum_{j=0}^{2 i}(-1)^{j} h_{j}$ é par então $\Gamma=\sum_{j=0}^{2 i}(-1)^{j+1} h_{j}-2 \sum_{j=i}^{2 i}(-1)^{j+1} h_{j}=$ $\sum_{j=0}^{i-1}(-1)^{j+1} h_{j}+\sum_{j=i}^{2 i}(-1)^{j} h_{j}$ também é, e neste caso, somos capazes de construir, como mostrado na seqüência, uma soluçāo $\gamma$ satisfazendo (4.38), (4.40) e tal que $\gamma_{i}$ é par.

Seja $h^{c d}=\left(h_{1}^{c}, h_{1}^{d}, \ldots, h_{i}^{c}, \beta, h_{i}^{d}, \ldots, h_{2 i-1}^{c}, h_{2 i-1}^{d}\right)$ uma solução inteira não negativa de (4.35). Se $\Gamma$ é par, então é fácil ver de (4.37) que $\beta$ é par. Assuma, sem perda de generalidade, que $h^{c d}$ satisfaz as equaçōes

$$
h_{j}^{c} h_{2 i-j}^{d}=0, \quad \text { for } j=2, \ldots, i \text {. }
$$

Para $2 \leq j \leq i-1$, o truque é o mesmo utilizado na prova da Proposição 4.1.2, isto é, adicione a uma solução arbitrária $h^{\text {cd }}$ de (4.35) a circulação apropriada ao longo do $(j-1)$ ésimo ciclo. Contudo, quando $j=i$, a solução do sistema homogêneo $\tilde{h}^{c d}$ que é adicionada a $h^{c d}$ é tal que $\left(\bar{h}_{i}^{c}, \bar{\beta}, \bar{h}_{i}^{d}\right)=\min \left\{h_{i}^{c}, h_{i}^{d}\right\}(-1,2,-1)$ e o restante das componentes são zero. Note que ambos os tipos de adição preservam a não negatividade e integralidade da solução de (4.35), enquanto a última, a única que pode modificar os valores das componentes $\beta$, preserva sua paridade.

Afirmamos que o vetor $\gamma$ definido por

$$
\begin{aligned}
& \gamma_{0}=\gamma_{2 i}=1 \\
& \gamma_{j}= \begin{cases}h_{j}^{d}-h_{j+1}^{c}, & \text { se } 1 \leq j \leq i-1 \\
\beta, & \text { se } j=i \\
-h_{j-1}^{d}+h_{j}^{c}, & \text { se } i+1 \leq j \leq 2 i-1\end{cases}
\end{aligned}
$$

é uma solução inteira não negativa de (4.38)-(4.40).

A integralidade de $h^{\text {cd }}$ implica a integralidade de $\gamma$. As cquações $2 i+1+j$, para $1 \leq j \leq i-1$, de (4.35) implicam

$$
\begin{aligned}
(-1)^{j}\left(h_{j}^{d}-h_{j+1}^{c}+h_{2 i-1-j}^{d}-h_{2 i-j}^{c}\right) & = \\
(-1)^{j}\left(\gamma_{j}-\gamma_{2 i-j}\right) & =0,
\end{aligned}
$$

o que significa que $\gamma$ satisfaz (4.40). A condição (4.39) é satisfeita por definição.

A não negatividade de $\gamma_{j}$ é trivial para $j=0, i$ e $2 i$, e, para $1 \leq j \leq 2 i-1$, segue de (4.40) e (4.41), como na prova da Proposição 4.1.2. Resta mostrar que $\gamma$ também 
satisfaz (4.38). A prova é análoga à prova da Proposição 4.1.2, assim é conveniente definir o sistema (4.44), equivalente a (4.34), obtido multiplicando-se as equações ímpares por -1 :

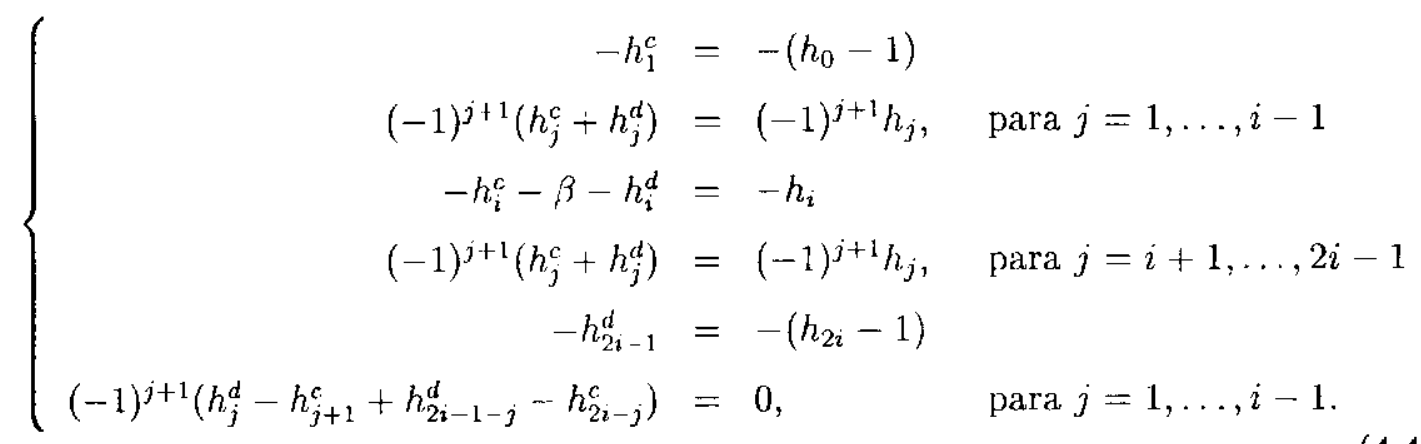

Somando as equações 1 até $2 i+1$ de (4.44) obtemos

$$
\begin{aligned}
-h_{1}^{c}+\sum_{j=1}^{2 i-1}(-1)^{j+1}\left(h_{j}^{c}+h_{j}^{d}\right)-h_{2 i-1}^{d}-\beta & = \\
h_{1}^{d}+\sum_{j=2}^{2 i-2}(-1)^{j+1}\left(h_{j}^{c}+h_{j}^{d}\right)+h_{2 i-1}^{c}-\beta & = \\
\sum_{j=1}^{2 i-2}(-1)^{j+1} h_{j}^{d}+\sum_{j=2}^{2 i-1}(-1)^{j+1} h_{j}^{c}-\beta & = \\
\sum_{j=1}^{2 i-2}(-1)^{j+1} h_{j}^{d}+\sum_{j=1}^{2 i-2}(-1)^{j} h_{j+1}^{c}-\beta & = \\
\sum_{j=1}^{2 i-2}(-1)^{j+1}\left(h_{j}^{d}-h_{j+1}^{c}\right)-\beta & =-\left(h_{0}-1\right)+\sum_{j=1}^{2 i \cdot 1}(-1)^{j+1} h_{j}-\left(h_{2 i}-1\right) \\
& =2+\sum_{j=0}^{2 i}(-1)^{j+1} h_{j} .
\end{aligned}
$$

A soma alternada dos $\gamma$ 's, de acordo com (4.42), resulta em

$$
\begin{aligned}
\sum_{j-0}^{2 i}(-1)^{j+1} \gamma_{j} & =-1+\sum_{j=1}^{i-1}(-1)^{j+1}\left(h_{j}^{d}-h_{j+1}^{c}\right)-\beta+\sum_{j=i+1}^{2 i-1}(-1)^{j+1}\left(-h_{j-1}^{d}+h_{j}^{c}\right)-1 \\
& =-2+\sum_{j=1}^{i-1}(-1)^{j+1}\left(h_{j}^{d}-h_{j+1}^{c}\right)+\sum_{j=i}^{2 i-2}(-1)^{j+1}\left(h_{j}^{d}-h_{j+1}^{c}\right)-\beta \\
& =-2+\sum_{j-1}^{2 i-2}(-1)^{j+1}\left(h_{j}^{d}-h_{j+1}^{c}\right)-\beta
\end{aligned}
$$


Comparando (4.45) e (4.46) concluímos que

$$
\sum_{j=0}^{2 i}(-1)^{j+1} \gamma_{j}=\sum_{j=0}^{2 i}(-1)^{j+1} h_{j},
$$

isto é, $\gamma$ satisfaz a primeira equação em (4.38).

Ao calcular a soma parcial das equações 1 até $2 i+1-\ell$ de (4.44), existem dois casos a considerar:

a) $1 \leq \ell \leq i$

$$
\begin{aligned}
-h_{1}^{c}+\sum_{j=1}^{2 i-\ell}(-1)^{j+1}\left(h_{j}^{c}+h_{j}^{d}\right)-\beta & = \\
h_{1}^{d}+\sum_{j=2}^{2 i-\ell}(-1)^{j+1}\left(h_{j}^{c}+h_{j}^{d}\right)-\beta & = \\
\sum_{j=1}^{2 i-\ell}(-1)^{j+1} h_{j}^{d}-\sum_{j=1}^{2 i-1-\ell}(-1)^{j+1} h_{j+1}^{c}-\beta & = \\
\sum_{j=1}^{2 i-1-\ell}(-1)^{j+1}\left(h_{j}^{d}-h_{j+1}^{c}\right)+(-1)^{2 i+1-\ell} h_{2 i-\ell}^{d}-\beta & =-\left(h_{0}-1\right)+\sum_{j=1}^{2 i-\ell}(-1)^{j+1} h_{j} \\
& =1+\sum_{j=0}^{2 i-\ell}(-1)^{j+1} h_{j} .
\end{aligned}
$$

b) $i+1 \leq \ell<2 i$

$$
\begin{aligned}
-h_{1}^{c}+\sum_{j=1}^{2 i-\ell}(-1)^{j+1}\left(h_{j}^{c}+h_{j}^{d}\right) & = \\
h_{1}^{d}+\sum_{j=2}^{2 i-\ell}(-1)^{j+1}\left(h_{j}^{c}+h_{j}^{d}\right) & = \\
\sum_{j=1}^{2 i-\ell}(-1)^{j+1} h_{j}^{d}-\sum_{j=1}^{2 i-1-\ell}(-1)^{j+1} h_{j+1}^{c} & = \\
\sum_{j=1}^{2 i-\ell}(-1)^{j+1}\left(h_{j}^{d}-h_{j+1}^{c}\right)+(-1)^{2 i+1 \cdot \ell} h_{2 i+1-\ell}^{c} & =-\left(h_{0}-1\right)+\sum_{j=1}^{2 i-\ell}(-1)^{j+1} h_{j} \\
& =1+\sum_{j=0}^{2 i \ell}(-1)^{j+1} h_{j} .
\end{aligned}
$$

Da mesma forma, separamos em duas possibilidades a soma parcial $\sum_{j=0}^{2 i-\ell}(-1)^{j+1} \gamma_{j}$ : 
a) $1 \leq \ell \leq i$

$$
\begin{aligned}
\sum_{j=0}^{2 i-\ell}(-1)^{j+1} \gamma_{j} & =-1+\sum_{j=1}^{i-1}(-1)^{j+1}\left(h_{j}^{d}-h_{j+1}^{c}\right)-\beta+\sum_{j=i+1}^{2 i-\ell}(-1)^{j+1}\left(-h_{j-1}^{d}+h_{j}^{c}\right) \\
& =-1+\sum_{j=1}^{i-1}(-1)^{j+1}\left(h_{j}^{d}-h_{j+1}^{c}\right)+\sum_{j=i}^{2 i-\ell-\ell}(-1)^{j+1}\left(h_{j}^{d}-h_{j+1}^{c}\right)-\beta \\
& =-1+\sum_{j=1}^{2 i-1-\ell}(-1)^{j+1}\left(h_{j}^{d}-h_{j+1}^{c}\right)-\beta
\end{aligned}
$$

Comparando (4.50) e (4.48) concluímos que

$$
\sum_{j=0}^{2 i-\ell}(-1)^{j+1} \gamma_{j}+(-1)^{2 i+1-\ell} h_{2 i-\ell}^{d}=\sum_{j=0}^{2 i-\ell}(-1)^{j+1} h_{j} .
$$

Usando o fato que $h_{j}^{d}>0$ para todo $j=1, \ldots, 2 i-1$, a cquação (4.51) implica

$$
\sum_{j=0}^{2 i-\ell}(-1)^{j+1} \gamma_{j} \begin{cases}\leq \sum_{j=0}^{2 i-\ell}(-1)^{j+1} h_{j}, & \text { se } 1 \leq \ell \leq i, \ell \text { odd } \\ \geq \sum_{j=0}^{2 i-\ell}(-1)^{j+1} h_{j}, & \text { se } 1 \leq \ell \leq i, \ell \text { par }\end{cases}
$$

o que significa que $\gamma$ definido em (4.42) satisfaz as desigualdades em (4.38), para $1 \leq \ell \leq i$.

b) $i+1<\ell \leq 2 i-1$

$$
\sum_{j=0}^{2 i-\ell}(-1)^{j+1} \gamma_{j}=-1+\sum_{j-1}^{2 i-\ell}(-1)^{j+1}\left(h_{j}^{d}-h_{j+1}^{c}\right) .
$$

Comparando (4.53) e (4.49) concluímos que

$$
\sum_{j-0}^{2 i-\ell}(-1)^{j+1} \gamma_{j}+(-1)^{2 i+1-\ell} h_{2 i+1-\ell}^{c}=\sum_{j=0}^{2 i-\ell}(-1)^{j+1} h_{j} .
$$

Usando o fato que $h_{j}^{c}>0$ para todo $j=1, \ldots, 2 i-1$, a equação (4.54) implica que $\gamma$ satisfaz (4.52) para $i+1 \leq \ell<2 i \cdots 1$.

Finalmente, de (4.49) com $\ell=2 i$ temos $-h_{1}^{c}=1-h_{0}$ e como, por hipótese, $h_{1}^{c} \geq 0$, então $-h_{0}<-1=-\gamma_{0}$, a última desigualdade em (4.38).

Assim, estabelecemos que $\gamma$ definido em (4.42) satisfaz as restrições em (4.38). 
Proposição 4.1.5 Se, para um dado conjunto de inteiros não negativos $\left(h_{0}, h_{1}, \ldots, h_{2 i}\right.$, $\left.h_{2 i}\right)$, existem inteiros não negativos $\gamma=\left(\gamma_{0}, \gamma_{1}, \ldots, \gamma_{2 i}\right)$ que satisfazem (4.38)-(4.40), então o sistema (4.34) tem uma solução inteira não negativa.

Demonstração: A prova é por construção. Considere o vetor $h^{c d}$ definido por

$$
\begin{array}{rlrl}
h_{2 i-\ell}^{d} & =(-1)^{\ell+1} \sum_{j=0}^{2 i-\ell}(-1)^{j+1}\left(h_{j}-\gamma_{j}\right), & \text { for } 1 \leq \ell \leq i \\
h_{2 i+1-\ell}^{c} & =(-1)^{\ell+1} \sum_{j=0}^{2 i-\ell}(-1)^{j+1}\left(h_{j}-\gamma_{j}\right), & \text { for } i+1 \leq \ell \leq 2 i \\
h_{\ell}^{d} & =\gamma_{\ell}+h_{\ell+1}^{c}, & & \text { para } 1 \leq \ell \leq i-1 \\
h_{\ell}^{c} & =\gamma_{\ell}+h_{\ell-1}^{d}, & & \text { para } i+1 \leq j \leq 2 i-1 \\
\beta & =\gamma_{i} . &
\end{array}
$$

Claramente, o vetor $h^{c d}$ dado por (4.55)-(4.59) é inteiro, já que $\gamma$ e $h$ o são. Reescrevendo (4.38) como

$$
(-1)^{k+1} \sum_{j=0}^{2 i \cdot k}(-1)^{j+1} \gamma_{j}\left\{\begin{array}{l}
=\sum_{j=0}^{2 i}(-1)^{j+1} h_{j}, \text { se } k=0 \\
\leq(-1)^{k+1} \sum_{j=0}^{2 i-k}(-1)^{j+1} h_{j}, \quad \text { se } 1 \leq k \leq 2 i
\end{array}\right.
$$

concluímos que as componentes de $h^{c d}$ definidas em (4.55) e (4.56) são não negativas. Este fato, por outro lado, juntamente com a hipótese $h \geq 0$ e $\gamma \geq 0$, implica que as componentes definidas em (4.57)-(4.59) são também não negativas.

Dc (4.56) $\operatorname{com} \ell=2 i$ obtemos

$$
h_{1}^{c}=(-1)^{2 i+1}(-1)^{0+1}\left(h_{0}-\gamma_{0}\right)=h_{0}-1
$$

isto é, a primeira equação em (4.34). Por outro lado, (4.55) com $\ell=1$ dá

$$
\begin{aligned}
h_{2 i-1}^{d} & =(-1)^{1+1} \sum_{j=0}^{2 i-1}(-1)^{j+1}\left(h_{j}-\gamma_{j}\right) \\
& =\sum_{j=0}^{2 i}(-1)^{j+1}\left(h_{j}-\gamma_{j}\right)-(-1)^{2 i+1}\left(h_{2 i}-\gamma_{2 i}\right) \\
& =h_{2 i}-1
\end{aligned}
$$

que é a $(2 i+1)$-ésima equação de (4.34). 
As últimas $i-1$ equações de (4.34) são facilmente verificadas:

$$
\begin{aligned}
h_{j}^{d}-h_{j+1}^{c}+h_{2 i-1-j}^{d}-h_{2 i-j}^{c} & =\left(\gamma_{j}+h_{j+1}^{c}\right)-h_{j+1}^{c}+h_{2 i-1-j}^{d}-\left(\gamma_{2 i-j}+h_{2 i-1-j}^{d}\right) \\
& =\gamma_{j}-\gamma_{2 i-j}=0, \quad \text { para } 1 \leq j \leq i-1 .
\end{aligned}
$$

Seja $i+1 \leq \ell \leq 2 i-1$. Somando as equações adequadas em (4.56) obtemos

$$
\begin{aligned}
h_{2 i+1-\ell}^{c}+h_{2 i-\ell}^{c} & =(-1)^{\ell+1}\left(\sum_{j=0}^{2 i-\ell}(-1)^{j+1}\left(h_{j}-\gamma_{j}\right)-\sum_{j-0}^{2 i-\ell-1}(-1)^{j+1}\left(h_{j}-\gamma_{j}\right)\right) \\
& =(-1)^{\ell+1}(-1)^{2 i+1-\ell}\left(h_{2 i-\ell}-\gamma_{2 i-\ell}\right) \\
& =h_{2 i \ell \ell}-\gamma_{2 i-\ell},
\end{aligned}
$$

o que implica, usando (4.57), a $(2 i+1-\ell)$-ćsima equação de(4.34):

$$
\gamma_{2 i-\ell}+h_{2 i+1-\ell}^{c}+h_{2 i-\ell}^{c}=h_{2 i-\ell}^{d}+h_{2 i-\ell}^{c}=h_{2 i \ell} \ell .
$$

Assim, as equaçōos $(1+j)$ de $(4.34)$, para $1 \leq j \leq i-1$, são satisfeitas por $h^{c d}$.

Verificando a $(i+1)$-ésima equação de (4.34):

$$
\begin{aligned}
h_{i}^{c}+\beta+h_{i}^{d} & =(-1)^{i+2} \sum_{j=0}^{2 i-(i+1)}(-1)^{j+1}\left(h_{j}-\gamma_{j}\right)+\gamma_{i}+(-1)^{i+1} \sum_{j=0}^{i}(-1)^{j+1}\left(h_{j}-\gamma_{j}\right) \\
& =(-1)^{i+1}(-1)^{i+1}\left(h_{i}-\gamma_{i}\right)+\gamma_{i} \\
& =h_{i} .
\end{aligned}
$$

Finalmente, seja $1 \leq \ell<i \ldots 1$. Temos

$$
\begin{aligned}
h_{2 i-\ell}^{d}+h_{2 i-1-\ell}^{d} & =(-1)^{\ell+1}\left(\sum_{j=0}^{2 i-\ell}(-1)^{j+l}\left(h_{j}-\gamma_{j}\right)-\sum_{j=0}^{2 i-1-\ell}(-1)^{j+1}\left(h_{j}-\gamma_{j}\right)\right) \\
& =(-1)^{\ell+1}(-1)^{2 i+1-\ell}\left(h_{2 i-\ell}-\gamma_{2 i-\ell}\right) \\
& =h_{2 i-\ell}-\gamma_{2 i-\ell},
\end{aligned}
$$

o que implica, usando (4.57)

$$
h_{2 i-\ell}^{d}+h_{2 i-1-\ell}^{d}+\gamma_{2 i-\ell}=h_{2 i-\ell}^{d}+h_{22 \ell}^{c}=h_{2 i-\ell},
$$

o que completa a verificação que $h^{\text {c.d }}$ satisfaz (4.34). 


\subsubsection{Caso $\mathrm{n}=2 \bmod 4$}

Nesta seção assumimos $n=2 i$, onde $i \geq 3$ c ímpar. Suponha que existam inteiros não negativos $\left\{h_{0}, h_{1}, \ldots, h_{2 i+1}, h_{1}^{c}, h_{1}^{d}, \ldots, h_{2 i}^{c}, h_{2 i}^{d}\right\}$ que satisfazem o sistema linear

$$
\left\{\begin{aligned}
h_{1}^{c} & =h_{0}-1 \\
h_{j}^{c}+h_{j}^{d} & =h_{j}, \quad \text { para } j=1, \ldots, 2 i-1 \\
h_{2 i-1}^{d} & =h_{2 i}-1 \\
h_{j}^{d}-h_{j+1}^{c}+h_{2 i-1-j}^{d}-h_{2 i-j}^{c} & =0, \quad \text { para } j=1, \ldots, i-1
\end{aligned}\right.
$$

Se o sistema (4.61) tem uma solução, então o sistema (4.62), obtido de (4.61) adicionando a variável $\delta$ na $i+1$-ésima equação, também tem, já que qualquer solução de (4.61) pode ser transformada em uma solução de (4.62) tomando $\delta$ zero.

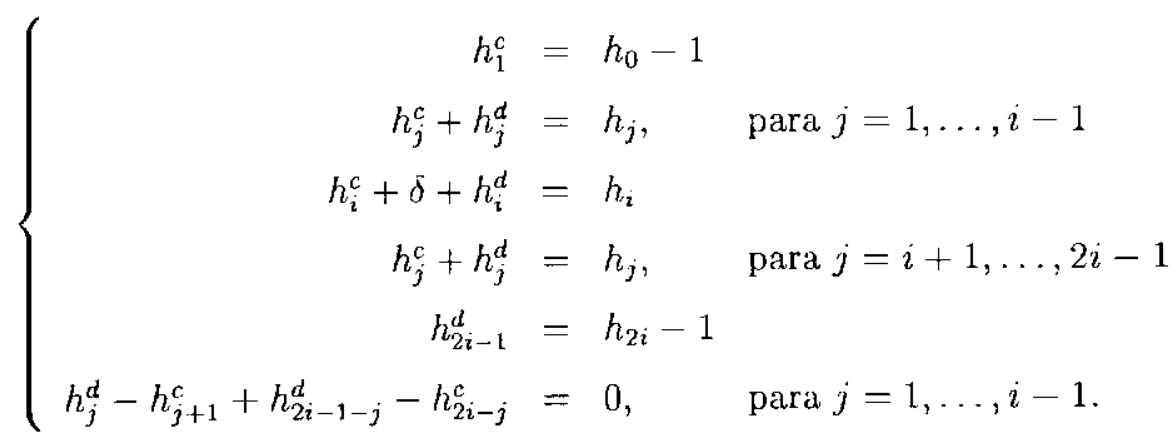

Note que o sistema (4.62) é do mesmo tipo que o sistema (4.34). Além disso, para (4.61) ter uma solução, devemos ter, conforme visto no Capítulo $3, \sum_{j=0}^{i-1}(-1)^{j+1} h_{j}+$ $\sum_{j=i}^{2 i}(-1)^{j} h_{j}$ par. Sc multiplicarmos por -1 as equações ímpares com índice no conjunto $\{1, \ldots, i\}$, e as equações pares com índices maiores, o sistema resultante, equivalente a (4.62), pode ser decomposto em dois sistemas lineares independentes. O primeiro é dado por (4.36) e o segundo por (4.63). Portanto, o primeiro sistema é um problema de fluxo de rede definido sobre um digrafo cuja matriz de incidência é a matriz de coeficientes de (4.4) para $n=2 i-1$. Este digrafo tem $i-2$ ciclos de comprimento quatro mais um caminho não orientado tambérn de comprimento quatro. O j-ésimo ciclo contém arcos associados às variáveis $h_{j+1}^{d}, h_{2 i-1-j}^{c}, h_{2 i-1}^{d} j_{j}$ e $h_{j+1}^{c}$, e a orientaçāo dos primeiros dois arcos é oposta à orientação dos dois últimos, com respeito a uma orientação arbitrária do ciclo. Nós do 
$j$-ésimo ciclo estão associados às equações $j+2,2 i+2+j, 2 i-j$ e $2 i+1+j$.

$$
\left\{\begin{aligned}
-h_{i}^{c}-\delta-h_{i}^{d} & =-h_{i} \\
-h_{i}^{c}+h_{i}^{d} & =\sum_{j=0}^{i-1}(-1)^{j+1} h_{j}+\sum_{j=i+1}^{2 i}(-1)^{j} h_{j}
\end{aligned}\right.
$$

Proposição 4.1.6 Se, para um dado conjunto de inteiros não negativos $\left(h_{0}, h_{1}, \ldots, h_{2 i}\right)$, o sistema (4.61) tem uma solução inteira não negativa, então existem inteiros não negativos $\left(\gamma_{0}, \gamma_{1}, \ldots, \gamma_{2 i}\right)$ satisfazendo

$$
\sum_{j=0}^{2 i-k}(-1)^{j+1} \gamma_{j}\left\{\begin{array}{l}
=\sum_{j=0}^{2 i}(-1)^{j+1} h_{j}, \quad \text { se } k=0 \\
\leq \sum_{j=0}^{2 i-k}(-1)^{j+1} h_{j}, \quad \text { se } 1 \leq k \leq 2 i, k \text { impar } \\
\geq \sum_{j=0}^{2 i-k}(-1)^{j+1} h_{j}, \quad \text { se } 1 \leq k \leq 2 i, k \text { par } \\
\gamma_{0}=\gamma_{2 i}=1 \\
\gamma_{j}=\gamma_{2 i-j}, \text { se } 1 \leq j \leq i-1
\end{array}\right.
$$

e tal que $\gamma_{i}$ é par.

Demonstração: Seja $h^{c d}=\left(h_{1}^{c}, h_{1}^{d}, \ldots, h_{i}^{c}, 0, h_{i}^{d}, \ldots, h_{2 i-1}^{c}, h_{2 i-1}^{d}\right)$ uma solução inteira não negativa de (4.62) obtida tomando uma solução inteira não negativa de (4.61) e estendendo-a a uma solução de (4.62) tomando $\delta$ zero. Empregando o argumento baseado na manipulação de circulações utilizado na prova da Proposição 4.1.2, podemos justificar a afirmação que $h^{\text {cd }}$ satisfaz as equações

$$
h_{j}^{c} h_{2 i-j}^{d}=0, \quad \text { para } j=2, \ldots, i-1 .
$$

Quando $j=i$, a solução para o sistema homogêneo $\tilde{h}^{c d}$ que é adicionada a $h^{c d}$ é tal que $\left(\tilde{h}_{i}^{c}, \tilde{\delta}, \tilde{h}_{i}^{d}\right)=\min \left\{h_{i}^{c}, h_{i}^{d}\right\}(-1,2,-1)$ e o restante das componentes são zero. Assim os valores de $\delta$ na solução considerada săo zero ou um número par positivo.

O restante da prova é análogo à prova da Proposição 4.1.4 de (4.42).

Proposição 4.1.7 Se, para um dado conjunto de inteiros não negativos $\left(h_{0}, h_{1}, \ldots, h_{2 i}, h_{2 i}\right)$, existem inteiros năo negativos $\gamma=\left(\gamma_{0}, \gamma_{1}, \ldots, \gamma_{2 i}\right)$, com $\gamma_{i}$ par, que satisfazem (4.64) (4.65), então o sistema (4.61) tem uma solução inteira näo negativa. 
Demonstração: Primeiro construímos uma solução inteira não negativa $\tilde{h}^{\text {cd }}=$ $\left(h_{1}^{c}, h_{1}^{d}, \ldots, h_{i}^{c}, \delta, h_{i}^{d}, \ldots, h_{2 i-1}^{c}, h_{2 i-1}^{d}\right)$ to $(4.62)$, com $\delta$ par:

$$
\begin{array}{rlrl}
h_{2 i \cdot \ell}^{d} & =(-1)^{\ell+1} \sum_{j=0}^{2 i-\ell}(-1)^{j+1}\left(h_{j}-\gamma_{j}\right), & \text { for } 1 \leq \ell \leq i \\
h_{2 i+1-\ell}^{c} & =(-1)^{\ell+1} \sum_{j=0}^{2 i-\ell}(-1)^{j+1}\left(h_{j}-\gamma_{j}\right), & \text { for } & i+1 \leq \ell \leq 2 i \\
h_{\ell}^{d} & =\gamma_{\ell}+h_{\ell+1}^{c}, & & \text { para } 1 \leq \ell \leq i-1 \\
h_{\ell}^{c} & =\gamma_{\ell}+h_{\ell-1}^{d}, & & \text { para } i+1 \leq j \leq 2 i-1 \\
\delta & =\gamma_{i} . &
\end{array}
$$

Como as equações de (4.62) têm a mesma estrutura que (4.34), a prova da Proposição 4.1.5 podem ser facilmente adaptada para mostrar que $\tilde{h}^{\text {cd }}$ é uma solução inteira não negativa de (4.62). A suposição que $\gamma_{i}$ é par implica que $\delta$ é par.

Finalmente, suponha $\delta=2 k$, onde $k$ é um inteiro não negativo. Afirmamos que $h^{c d}=\left(h_{1}^{c}, h_{1}^{d}, \ldots, h_{i}^{c}+k, h_{i}^{d}+k, \ldots, h_{2 i-1}^{c}, h_{2 i-1}^{d}\right)$ é uma solução inteira não negativa de (4.61). Para ver isto precisamos apenas verificar as equações contendo $h_{i}^{c}$ e $h_{i}^{d}$ :

$$
\begin{aligned}
h_{i} & =h_{i}^{c}+\delta+h_{i}^{d}=h_{i}^{c}+2 k+h_{i}^{d}=\left(h_{i}^{c}+k\right)-\left(h_{i}^{d}+k\right), \\
0 & =h_{i-1}^{d}-h_{i}^{c}+h_{i}^{d}-h_{i+1}^{c}=h_{i-1}^{d}-h_{i}^{c}-k+k+h_{i}^{d}-h_{i+1}^{c}= \\
& =h_{i-1}^{d}-\left(h_{i}^{c}+k\right)+\left(h_{i}^{d}+k\right)-h_{i+1}^{c} .
\end{aligned}
$$

\subsection{Politopos}

Nesta seção estudaremos o poliedro $\mathcal{P}$ constituído pelas soluçōes não negativas de (1) para um dado de índices pré-fixados $\left(h_{0}, \ldots, h_{n}\right)$, que satisfazem as condições de dualidade $\gamma_{k}=\gamma_{n-k}$, para $k=0, \ldots, n$.

Primeiramente mostramos que $\mathcal{P}$ é um politopo, i.e., um poliedro limitado. Isto segue da restrição de não negatividade sobre $\gamma$ e do fato que, somando todos os possíveis pares de desigualdades consecutivas em (1), concluímos $\gamma_{j} \leq h_{j}$, para $j=1, \ldots, n$.

Além disso, mostramos que este politopo tem vértices inteiros, o que implica que $\mathcal{P}$ é o casco convexo dos vetores inteiros em $\mathcal{P}$. ou, equivalentemente, $\mathcal{P}$ é o casco convexo da 
coleção dos vetores de números de Betti que satisfazem as desigualdades de Morse (1).

\subsubsection{Caso $n$ ímpar}

As Proposiçōes 4.1.2 e 4.1 .3 não somente estabelecem que (4.3) tem uma solıção se, e somente sc, (4.5) -(4.7) também tem uma, elas também mostram como construir um $\gamma$ não negativo satisfazendo (4.5) (4.7) de $u m h^{c d}$ não negativo apropriado satisfazendo (4.3) e vice-versa. Isto sugere a existência de algum tipo de relacionamento entre os poliedros definidos pelos dois sistemas de desigualdades.

No Capítulo 3 mostramos que o poliedro composto dos $h^{\text {ct }}$ não negativos satisfazendo (4.3) é um politopo inteiro, isto é, um poliedro limitado com vértices inteiros. Mais ainda, do desenvolvimento neste capítulo podemos extrair uma "receita"para construir todos os $h^{\text {cd }}$ inteiros não negativos deste politopo.

Mostraremos nesta seção que propriedades similares se mantêm para o politopo de $\gamma$ 's não negativos satisfazendo (4.5)-(4.7). Além disso, o conhecimento ganho sobre a geração de $l^{c d}$ viáveis no Capítulo 3 , a construção exibida na prova da Proposição 4.1.3, e as características que serão estabelecidas para este politopo, levam a um mecanismo para construir todos os $\gamma$ 's pertencentes a este politopo.

Para simplificar a exposição, as condições (4.6)-(4.7) serão usadas para eliminar mais da metade das variáveis, a saber $\gamma_{0}, \gamma_{i+1}, \ldots, \gamma_{2 i+1}$. Usando as cquaçōes (4.6)-(4.7), temos que

$$
\sum_{j=0}^{2 i+1-k}(-1)^{j+1} \gamma_{j}=-1+\sum_{j=1}^{\min \{k-1,2 i+1-k\}}(-1)^{j+1} \gamma_{j}
$$

Também note que as condições (4.6) e (4.7) implicam que as restrições correspondendo a $k=0,1$ e $2 i+1$ em (4.5) representam, na verdade, restrições sobre o vetor (fixado) $h=$ $\left(h_{0}, \ldots, h_{n}\right)$. Existc uma correspondência 1-a-1 entre $\gamma=\left(\gamma_{0}, \ldots, \gamma_{2 i+1}\right)$ 's não negativos satisfazendo (4.5)-(4.7) e $\gamma^{r}=\left(\gamma_{1}, \ldots, \gamma_{i}\right)$ 's não negativos satisfazendo (4.72) abaixo. Assim, ao invés de $\mathcal{P}$, podemos considerar o politopo $\mathcal{P}^{r}=\left\{\gamma^{r} \in \mathbb{R}^{i} \mid\right.$ restrições em (4.72) $\}$. 


$$
\begin{gathered}
0=\sum_{j=0}^{2 i+1}(-1)^{j+1} h_{j}, \quad 0 \leq h_{0}-1, \quad 0 \leq h_{2 i+1}-1 \\
\sum_{j=1}^{\min \{k-1,2 i+1 \cdot k\}}(-1)^{j+1} \gamma_{j} \leq 1+\sum_{j=0}^{2 i+1-k}(-1)^{j+1} h_{j}, \text { for } 2 \leq k \leq 2 i, k \text { even } \\
\sum_{j=1}^{\min \{k-1,2 i+1-k\}}(-1)^{j+1} \gamma_{j} \geq 1+\sum_{j=0}^{2 i+1-k}(-1)^{j+1} h_{j}, \text { para } 2 \leq k \leq 2 i, k \text { ímpar } \\
\gamma^{r} \geq 0 .
\end{gathered}
$$

Proposiçāo 4.2.1 O politopo $\mathcal{P}^{r}$ dado por (4.72) satisfaz as seguintes propriedades:

1. Os vértices de $\mathcal{P}^{r}$ são inteiros.

2. Cada vértice de $\mathcal{P}^{r}$ pertence a uma das seguintes faces: $\mathcal{F}_{t}=\left\{\gamma \in \mathcal{P}^{r}\right.$ $\left.\sum_{j=1}^{i}(-1)^{j+1} \gamma_{j}=1+\sum_{j=1}^{i}(-1)^{j+1} h_{j}\right\}$ ou $\mathcal{F}_{0}=\left\{\gamma \in \mathcal{P}^{r} \mid \gamma_{i}=0\right\}$.

3. Cada $\gamma^{r}$ não negativo (inteiro) em $\mathcal{F}_{t}$ corresponde a um $h^{\text {cd }}$ não negativo inteiro satisfazendo (4.3).

\section{Demonstração:}

1. Seja $A$ a matriz de coeficientes do sistema de desigualdades em $\mathcal{P}^{r}$, com exceção das restrições de não negatividade. A variável $\gamma_{j}$ aparece apcnas na linhas $3+j$ até $3+2 i-j$, com coeficient,es $(-1)^{j+1}$. Assim a matriz $0,1 \bar{A}=\left(\tilde{a}_{i j}\right)=\left(\left|a_{i j}\right|\right)$ tem a propriedade de 1's consecutivos, o que implica que é totalmente unimodular, ver $[13,15]$. Como $A$ é obtida de $\tilde{A}$ multiplicando colunas pares por $-1, A$ é também totalmente unimodular. Finalmente note que os elementos do lado direito nas desigualdades que definem o politopo $\mathcal{P}^{r}$ são claramente inteiros. Assim, o politopo $\mathcal{P}^{r}$ definido por (4.72) tem vértices inteiros, ou equivalentemente, é o casco convexo dos vetores inteiros satisfazendo as desigualdades em (4.72).

2. Seja $\bar{\gamma}^{r} \in \mathcal{P}^{r}$ e seja $M \bar{\gamma}^{r}=q$ o conjunto de restrições que estão saturadas em $\bar{\gamma}^{r}$. Então $\bar{\gamma}^{r}$ é vértice de $\mathcal{P}$ se, e somente se, o posto de $M$ é igual a $i$. Mas $\bar{A}$ contém precisamente $i$ colunas, o que implica que $M$ não pode conter uma colına de zeros. Como existem apenas duas desigualdades contendo $\gamma_{i}$, pelo menos uma delas deve estar saturada em $\bar{\gamma}$, caso contrário a $i$-ésima coluna de $M$ será o vetor 
nulo. Portanto se $\bar{\gamma}$ é um vértice ele deve pertencer a uma das faces $\mathcal{F}_{t}=\left\{\gamma \in \mathcal{P}^{r}\right.$ $\left.(-1)^{i+1} \sum_{j=1}^{i}(-1)^{j+1} \gamma_{j} \leq(-1)^{i+1}\left(1+\sum_{j=1}^{i}(-1)^{j+1} h_{j}\right)\right\}$ ou $\mathcal{F}_{0}=\left\{\gamma \in \mathcal{P}^{r} \mid \gamma_{i}=0\right\}$.

3. Se $\gamma^{r} \in \mathcal{P}^{r}$ (inteiro) pertence a $\mathcal{F}_{t}$, então $\gamma$ não negativo dado por $\gamma_{0}=\gamma_{2 i+1}=$ 1 e $\gamma_{2 i+1-j}=\gamma_{j}$, para $j=1, \ldots, i$ satisfaz (4.5) (4.7) e satura a desigualdade correspondente a $k=i+1 \mathrm{em}(4.5)$. Mas então $h^{c \cdot d}$ dado pelas equações (4.27)(4.32) é um vetor nāo negativo (inteiro) satisfazendo (4.3), como mostrado na prova da Proposição 4.1.3.

Suponha que o sistema de equaçōes (4.4) admite soluções não negativas. Seja $\tilde{h}^{\text {cd1 }}$ a solução inteira não negativa que também satisfaz as condições de complementaridade dadas em (4.8). Seja $\bar{\gamma}=\left(\tilde{\gamma}_{0}, \tilde{\gamma}_{1}, \ldots, \tilde{\gamma}_{2 i+1}\right)$ a solıção inteira não negativa de (4.5) (4.7) dada por (4.9). Sua restrição $\tilde{\gamma}^{r}$ representa um papel especial em $\mathcal{P}^{r}$, como evidenciado na próxima proposião.

Proposição 4.2.2 Suponha que o sistema de equaçöes (4.4) admite soluções não negativas. Então o politopo $\mathcal{P}^{r}$ pode ser reescrito como

$$
\mathcal{P}^{r}=\left\{0 \leq \gamma^{r} \in \mathbb{R}^{i} \mid(-1)^{k+1} \sum_{j=0}^{k}(-1)^{j+1} \gamma_{j} \leq(-1)^{k+1} \sum_{j=0}^{k}(-1)^{j+1} \tilde{\gamma}_{j}, \text { for } 1 \leq k \leq i\right\} .
$$

Além disso, $\tilde{\gamma}^{r}$ é um vértice de $\mathcal{P}^{r}$ e também seu vetor máximo, componente-a-componente.

Demonstração: Se (4.4) admite soluções não negativas, as restrições $0=$ $\sum_{j=0}^{2 i+1}(-1)^{j+1} h_{j}, 0 \leq h_{0}-1$ e $0 \leq h_{2 i+1}-1$ são redundantes e podem ser descartadas. Agora note que

$$
\min \{k-1,2 i+1-k\}= \begin{cases}k-1, & \text { se } 2 \leq k \leq i \\ i=k \cdots 1=2 i+1-k, & \text { se } k=i+1 \\ 2 i+1-k, & \text { se } i+2 \leq k \leq 2 i\end{cases}
$$

Portanto, a soma parcial $\sum_{j=1}^{\ell}(-1)^{j+1} \gamma_{j}$, para $1 \leq \ell \leq i-1$, aparece duas vezes em (4.72), :quando $k=\ell+1$ e quando $k=2 i+1-\ell$. Como $\ell+1$ e $2 i+1-\ell$ são ou ambos

\footnotetext{
${ }^{1}$ Se as condições de complementaridade (4.8) estão satisfeitas então o subgrafo induzido pelo suporte de $\tilde{h}^{\text {cd }}$ é uma floresta, o que implica que $\tilde{h}^{\text {cd }}$ é a única solução com um tal suporte.
} 
ímpares ou ambos pares, podemos juntar estas duas desigualdades como segue:

$\sum_{j=1}^{\ell}(-1)^{j+1} \gamma_{j} \begin{cases}\geq 1+\max \left\{\sum_{j=0}^{2 i-\ell}(-1)^{j+1} h_{j}, \sum_{j=0}^{\ell}(-1)^{j+1} h_{j}\right\}, & \text { se } 1 \leq \ell \leq i-1, \ell \text { par } \\ \leq 1+\min \left\{\sum_{j=0}^{2 i-\ell}(-1)^{j+1} h_{j}, \sum_{j=0}^{\ell}(-1)^{j+1} h_{j}\right\}, & \text { se } 1 \leq \ell \leq i-1, \ell \text { ímpar. }\end{cases}$

O fato que $\tilde{\gamma}$ é uma solução inteira não negativa de (4.5) (4.7) implica que $\bar{\gamma}^{r} \in \mathcal{P}^{r}$. Além disso, usando (4.7), as equaçōes (4.17) e (4.22), para $2 \leq \ell \leq i$ e $k=2 i+2-\ell$ (assim $i+2 \leq k \leq 2 i)$, obternos

$$
\begin{gathered}
\sum_{j=0}^{2 i+1-\ell}(-1)^{j+1} \tilde{\gamma}_{j}+(-1)^{2 i+2-\ell} \tilde{h}_{2 i+1-\ell}^{d}=\sum_{j=0}^{\ell-1}(-1)^{j+1} \tilde{\gamma}_{j}+(-1)^{2 i+2-\ell} \tilde{h}_{2 i+1-\ell}^{d}=\sum_{j=0}^{2 i+1-\ell}(-1)^{j+1}(4 A .74) \\
\sum_{j=0}^{2 i+1-k}(-1)^{j+1} \tilde{\gamma}_{j}+(-1)^{2 i+2-k} \tilde{h}_{2 i+2-k}^{c}=\sum_{j=0}^{\ell-1}(-1)^{j+1} \tilde{\gamma}_{j}+(-1)^{\ell} \tilde{h}_{\ell}^{c}=\sum_{j=0}^{\ell-1}(-1)^{j+1} h f(4.75)
\end{gathered}
$$

Como, por (4.8), $\tilde{h}_{\ell}^{c} \tilde{h}_{2 i+1-\ell}^{d}=0$, pelo menos uma das duas desigualdades acima envolvendo a soma parcial $\sum_{j=0}^{\ell-1}(-1)^{j+1} \tilde{\gamma}_{j}$, deve ser satisfeita como igualdade. Assim $\tilde{\gamma}^{r}$ satisfaz:

$$
\sum_{j=0}^{\ell-1}(-1)^{j+1} \bar{\gamma}_{j}= \begin{cases}\max \left\{\sum_{j=0}^{2 i+1-\ell}(-1)^{j+1} h_{j}, \sum_{j=0}^{\ell} 1(\cdots-1)^{j+1} h_{j}\right\}, & \text { se } 2 \leq \ell \leq i, \ell \text { impar } \\ \min \left\{\sum_{j=0}^{2 i+1-\ell}(-1)^{j+1} h_{j}, \sum_{j=0}^{\ell-1}(-1)^{j+1} h_{j}\right\}, & \text { se } 2 \leq \ell \leq i, \ell \text { par }\end{cases}
$$

o que implica (4.73) para $k \neq i$, levando em conta que $\tilde{\gamma}_{0}=1$. Finalmente, a desigualdade em (4.72) correspondendo a $k=i$ é satisfeita como igualdade por $\tilde{\gamma}$, como visto em (4.20) e $\operatorname{assim}$

$$
\sum_{j=0}^{i}(-1)^{j+1} \tilde{\gamma}_{j}=\sum_{j=0}^{i}(-1)^{j+1} h_{j}
$$

Os casos pares e ímpares podem ser combinados pela multiplicação adequada, produzindo as desigualdades desejadas, que, juntamente com as restrições de não negatividade, produzem uma definição alternativa para $\mathcal{P}^{r}$ :

$$
\begin{aligned}
(-1)^{\ell+1} \sum_{j=1}^{\ell}(-1)^{j+1} \gamma_{j} & <(-1)^{\ell+1} \sum_{j=1}^{\ell}(-1)^{j+1} \bar{\gamma}_{j}, \quad \text { para } 1 \leq \ell \leq i \\
\gamma^{r} & >0
\end{aligned}
$$

Isto completa a prova da equivalência entre (4.72) e (4.73).

Finalmente, a primeira desigualdade $\operatorname{cm}(4.77)$ dá $\gamma_{1}<\tilde{\gamma}_{1}$, e, se acrescentamos as desigualdades $j-1$ e $j$ de (4.77) obtemos $\gamma_{j} \leq \tilde{\gamma}_{j}$. Assim $\tilde{\gamma}^{r}$ é o vetor máximo, componentea-componente, de $\mathcal{P}$. Então $\tilde{\gamma}^{r}$ deve ser um vértice de $\mathcal{P}^{r}$. 
A Proposição 4.2.1 e o argumento na prova da Proposição 4.1 .3 expressada em (4.26) implica que $\mathcal{F}_{0}$ é a projeção de $\mathcal{F}_{t}$ sobre o hiperplano $\gamma_{i}=0$, e $\mathcal{P}$ é o casco convexo de $\mathcal{F}_{t} \cup \mathcal{F}_{0}$. Também pela Proposição 4.2.1, cada vetor (inteiro) em $\mathcal{F}_{t}$ pode ser obtido de um $h^{c d}$ correspondente satisfazendo (4.3) (ou, equivalentemente, (4.4)), embora isto não venha a constituir, em geral, uma correspondência 1-a-1. Agora, dada uma solução particular inteira $\tilde{h}^{\text {cd }}$, que satisfaça (4.8), podemos construir todos os vetores inteiros em $\mathcal{F}_{l}$ pela adição sucessiva de circulações à solução particular e cálculo do $\gamma^{r}$ correspondente. O seguinte exemplo ilustra estes fatos em um contexto concreto.

Exemplo Seja $n=2 i+1=7,\left(h_{0}, \ldots, h_{7}\right)=(2,5,11,10,5,3,3,3)$. Usando fórmulas deselvolvidas no Capítulo 3 para a solução geral $h^{\text {cd }}$ de (4.4) podemos obter a solução (particular) $\tilde{h}^{\text {cd }}$ que satisfaz $(4.8), \tilde{h}^{\text {cd }}=(1,4,3,8,5,5,5,0,3,0,1,2)$, exibida na Figura 4.2. Todas as outras soluções inteiras não negativas de (4.4) podem ser obtidas acrescentando-se múltiplos inteiros da circulação unitária ao longo do ciclo 1 , $h^{c d !}=(0,0,1,-1,0,0,0,0,-1,1,0,0)$, e/ou da circulação unitária ao longo do ciclo 2 , $h^{c d 2}=(0,0,0,0,1,-1,-1,1,0,0,0,0)$. De $\tilde{h}^{c t}$ obtemos o vetor $\tilde{\gamma}=(1,1,3,5,5,3,1,1), \mathrm{e}$, usando a Proposição 4.2.2, o politopo $\mathcal{P}^{r}$ é dado pelas desigualdades

$$
\begin{aligned}
\gamma_{1} & \leq 1 \\
\gamma_{1}-\gamma_{2} & \geq-2 \\
\gamma_{1}-\gamma_{2}+\gamma_{3} & \leq 3 \\
\gamma_{1}, \gamma_{2}, \gamma_{3} & \geq 0
\end{aligned}
$$

A Figura 4.3 contém quatro vistas do politopo $\mathcal{P}^{r}$. Neste exemplo nenhuma desigualdades é redundante, e o politopo tem seis faces. A face $\mathcal{F}_{0}$ está sempre escondida, mas podemos ver com a ajuda da Figura 4.4, que esta face é a projeção da face do topo $\mathcal{F}_{l}$ sobre o plano $\gamma_{3}=0$. O vetor máximo $\bar{\gamma}^{r}$ está rotulado sobre a primeira vista de $\mathcal{P}^{r}$.

Para ver a relação entre as soluções $h^{c d}$ obtidas como somas de circulações e o $\gamma^{r}$ correspondente, é mais conveniente olhar para o "esqueleto" do politopo $\mathcal{P}^{r}$, descrito na Figura 4.4(a), juntamente com o reticulado determinada pelos vetores inteiros no quadrante não negativo. Os pontos inteiros não negativos pertencentes a $\mathcal{P}^{r}$ são enfatizados. Denote por $\gamma^{1}=\tilde{\gamma}^{r}, \gamma^{2}, \ldots, \gamma^{7}$ os vetores inteiros pertencentes a $\mathcal{F}_{t}$, no sentido antihorário. Note que acrescentar $h^{\text {cd } 1}$ a $h^{c d}$ tem o efeito de subtrair 1 de $\gamma_{1}$ e $\gamma_{2}$. Similarmente, 


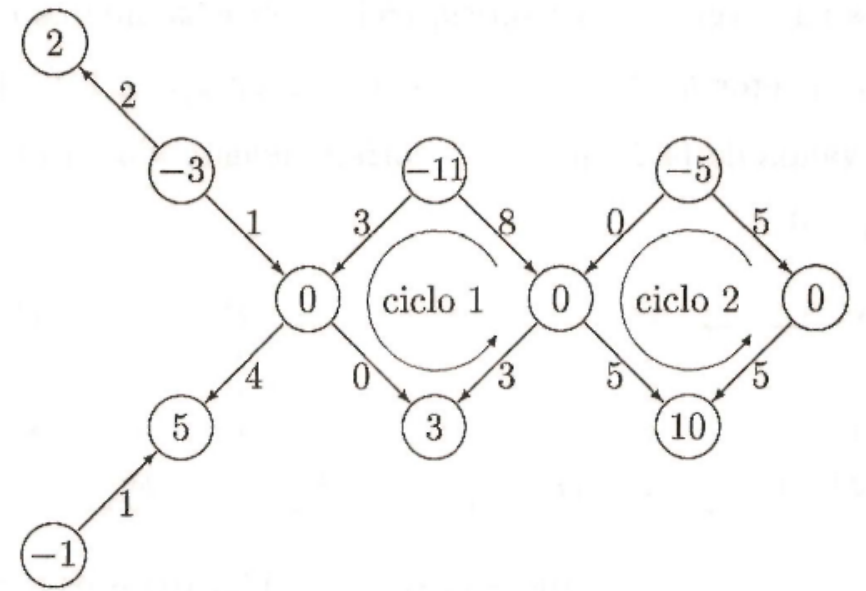

Figura 4.2: Solução $\tilde{h}^{c d}$ do exemplo.
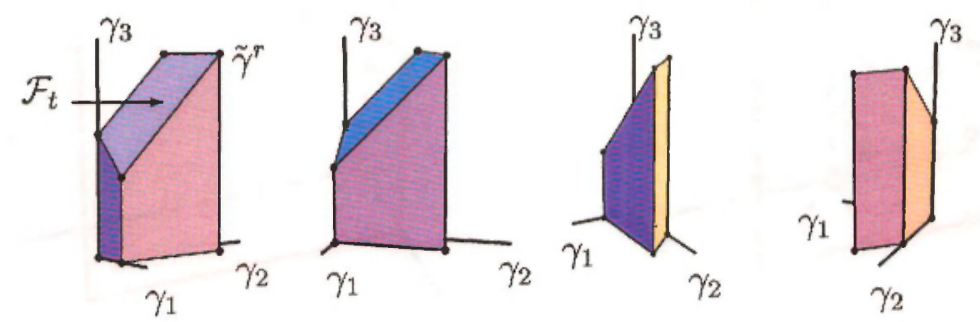

Figura 4.3: Quatro vistas do politopo $\mathcal{P}^{r}$. 
acrescentar $h^{c d 2}$ a $h^{\text {cd }}$ tem o efeito de subtrair 1 de $\gamma_{2}$ e $\gamma_{3}$. A Figura 4.4(b) focaliza $\mathcal{F}_{t}$ e seus pontos inteiros. O seguinte diagrama resume as operações com circulações sobre $h^{\text {cd }}$ necessárias para gerar todos os pontos em $\mathcal{F}_{t}$. Note que não existe uma correspondência 1-a-1 entre os inteiros não negativos $h^{\text {cd }}$ satisfazendo (4.4) e os inteiros não negativos $\gamma^{r}$ em $\mathcal{F}_{t}$. Por exemplo, o vetor $h^{\text {cd(4) }}+h^{\text {cd } 2}=(1,4,4,7,8,2,2,3,2,1,1,2)$ é uma solução inteira não negativa válida de (4.4), mas o $\gamma^{r}$ correspondente viola as restrições de não negatividade, pois $\gamma_{2} \geq 0$.

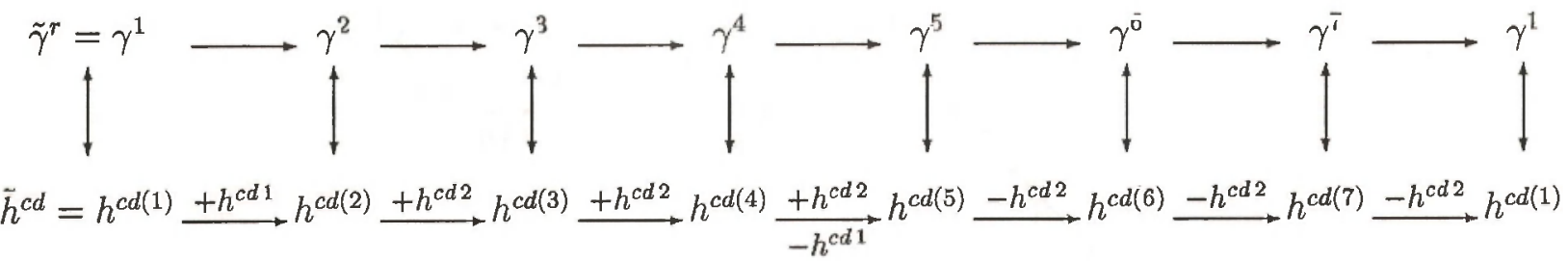

O conhecimento de $\gamma^{1}, \ldots, \gamma^{7}$ de fato torna possível calcular todos os pontos inteiros em $\mathcal{P}^{r}$, já que eles ficam em um dos segmentos $\left[\gamma^{i}, \hat{\gamma}_{i}\right]$, para $i=1, \ldots, 7$, onde $\dot{\gamma}_{j}^{i}=\gamma_{j}^{i}$, para $j \neq 3$, e $\dot{\gamma}_{3}=0$.

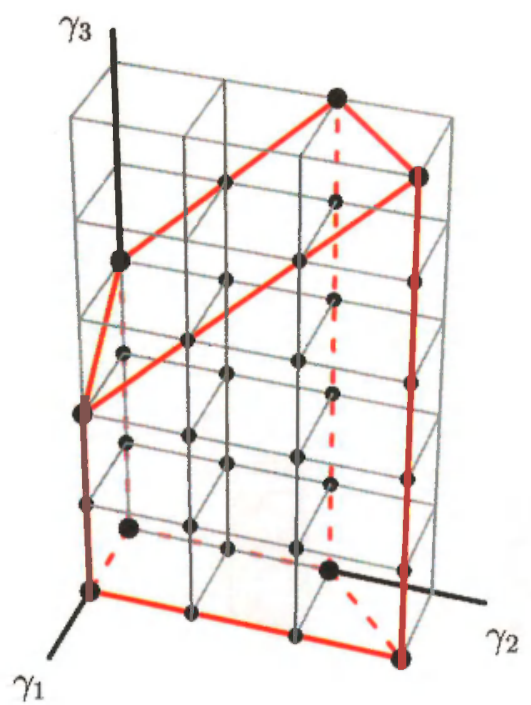

(a) Grade dos pontos inteiros em $\mathcal{P}^{r}$.

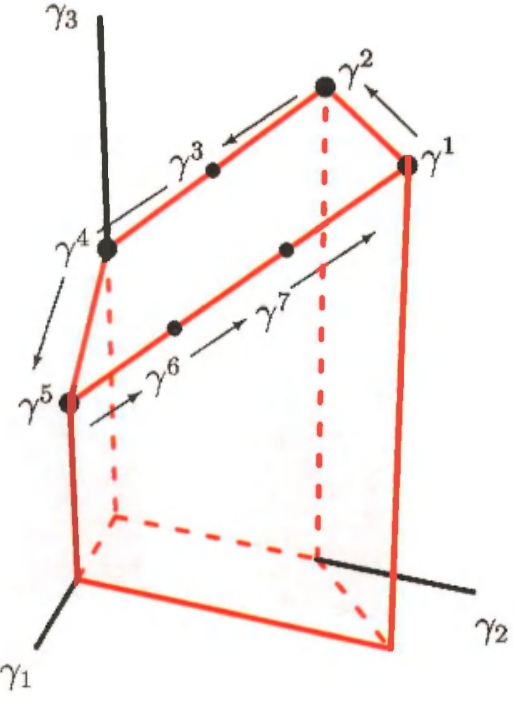

(b) Pontos inteiros na face $\mathcal{F}_{t}$ de $\mathcal{P}^{r}$.

Figura 4.4: Esqueleto do politopo $\mathcal{P}^{r}$. 


\subsubsection{Caso $\mathrm{n}=0 \bmod 4$}

Suponha $n=2 i$, onde $i \geq 2$ é par. Considere o sistema linear em $\gamma=\left\{\gamma_{0}, \gamma_{1}, \ldots, \gamma_{2 i}\right\}$ constituído por (4.38) (4.40) e as restrições de não negatividade (4.78) abaixo.

$$
\gamma \geq 0
$$

onde o vetor $\left(h_{0}, \ldots, h_{2 i}\right)$ é tal que $\sum_{j=0}^{2 i}(-1)^{i} h_{j}$ é par.

A primeira desigualdade em (4.38) pode ser usada para eliminar $\gamma_{i}$ do sistema. Isto é realizado ao substituir

$$
\gamma_{i}=(-1)^{i+1}\left(\sum_{j=0}^{2 i}(-1)^{j+1} h_{j}-\sum_{j=0}^{i-1}(-1)^{j+1} \gamma_{j}-\sum_{j=i+1}^{2 i}(-1)^{j+1} \gamma_{j}\right) .
$$

nas equaçöes contendo $\gamma_{i}$ em (4.38), i.e., aquelas correspondentes a $k=1, \ldots, i$. A restrição correspondente a um $k \in\{1, \ldots, i\}$ genérico é transformada como segue:

$$
\begin{aligned}
& \left.(-1)^{k+1}\left(\sum_{\substack{j=0 \\
j \neq i}}^{2 i-k}(-1)^{j+1} \gamma_{j}+(-1)^{2(i+1)}\left(-\sum_{j=0}^{i-1}(-1)^{j+1} \gamma_{j}-\sum_{j=i+1}^{2 i}(-1)^{j+1} \gamma_{j}\right)\right)\right) \\
= & -(-1)^{k+1} \sum_{j=2 i+1-k}^{2 i}(-1)^{j+1} \gamma_{j} \\
\leq & (-1)^{k+1}\left(\sum_{j=0}^{2 i-k}(-1)^{j+1} h_{j}-(-1)^{2(i+1)} \sum_{j=0}^{2 i}(-1)^{j+1} h_{j}\right) \\
= & -(-1)^{k+1} \sum_{j=2 i+1-k}^{2 i}(-1)^{j+1} h_{j}
\end{aligned}
$$

Usando (4.40), as restrições acima tornam-se

$$
\begin{aligned}
-(-1)^{k+1} \sum_{j=2 i+1-k}^{2 i}(-1)^{j+1} \gamma_{j} & =-(-1)^{k+1} \sum_{j=2 i+1-k}^{2 k}(-1)^{j+1} \gamma_{2 i-j} \\
& =-(-1)^{k+1} \sum_{j=0}^{k-1}(-1)^{j+1} \gamma_{j} \\
& \leq-(-1)^{k+1} \sum_{j=2 i+1-k}^{2 i}(-1)^{j+1} h_{j},
\end{aligned}
$$


e $\gamma_{i} \geq 0$ implica

$$
\begin{aligned}
(-1)^{i+1}\left(\sum_{j=0}^{2 i}(-1)^{j+1} h_{j}-\sum_{j=0}^{i-1}(-1)^{j+1} \gamma_{j}-\sum_{j=i+1}^{2 i}(-1)^{j+1} \gamma_{j}\right) & = \\
(-1)^{i+1}\left(\sum_{j=0}^{2 i}(-1)^{j+1} h_{j}-\sum_{j=0}^{i-1}(-1)^{j+1} \gamma_{j}-\sum_{j=i+1}^{2 i}(-1)^{j+1} \gamma_{2 i-j}\right) & = \\
(-1)^{i+1}\left(\sum_{j=0}^{2 i}(-1)^{j+1} h_{j}-\sum_{j=0}^{i-1}(-1)^{j+1} \gamma_{j}-\sum_{\ell=0}^{i-1}(-1)^{j+1} \gamma_{\ell}\right) & = \\
(-1)^{i+1}\left(\sum_{j=0}^{2 i}(-1)^{j+1} h_{j}-2 \sum_{j=0}^{j=1}(-1)^{j+1} \gamma_{j}\right) & \geq 0 .
\end{aligned}
$$

Portanto existe uma correspondência 1-a-1 entre as soluçōes $\gamma=\left(\gamma_{0}, \gamma_{1}, \ldots, \gamma_{2 i}\right)$ de (4.38)-(4.78) e as soluções $\gamma^{r}=\left(\gamma_{1}, \ldots, \gamma_{i-1}\right)$ de (4.80). Assim, podcmos simplificar nosso estudo de $\mathcal{P}$ considerando, em seu lugar, $\mathcal{P}^{r}=\left\{\gamma^{r} \in \mathbb{R}^{i-1} \mid\right.$ restrições em (4.80) $\}$.

$$
\begin{aligned}
& 0>1-h_{2 i} \\
& \sum_{j=1}^{k}(-1)^{j+1} \gamma_{j}\left\{\begin{array}{l}
\leq 1+\sum_{j=2 i-k}^{2 i}(-1)^{j+1} h_{j}, \quad \text { se } 1<k \leq i-1, k \text { impar } \\
\geq 1+\sum_{j=2 i-k}^{2 i}(-1)^{j+1} h_{j}, \quad \text { se } 1 \leq k \leq i-1, k \text { par }
\end{array}\right. \\
& (-1)^{i} \sum_{j-1}^{i-1}(-1)^{j+1} \gamma_{j} \geq(-1)^{i}\left(1+\frac{1}{2} \sum_{j=0}^{2 i}(-1)^{j+1} h_{j}\right) \\
& \sum_{j=1}^{2 i-k}(-1)^{j+1} \gamma_{j} \begin{cases}\leq 1+\sum_{j=0}^{2 i-k}(-1)^{j+1} h_{j}, & \text { se } i+1 \leq k \leq 2 i-1, k \text { ímpar } \\
\geq 1+\sum_{j-0}^{2 i-k}(-1)^{j+1} h_{j}, & \text { se } i+1 \leq k<2 i-1, k \text { par }\end{cases} \\
& 0 \geq 1-h_{0} \\
& \gamma_{j}>0, \quad \text { for } 1 \leq j \leq i-1
\end{aligned}
$$

Proposição 4.2.3 O politopo $\mathcal{P}^{r}$ definido por (4.80) tem vértices inteiros e cada $\gamma^{r}$ (inteiro) no politopo corresponde a um $h^{\text {cd }}$ näo negativo (inteiro) satisfazendo (4.34). Cada 
vértice de $\mathcal{P}^{r}$ pertence a uma das três faces:

$$
\begin{aligned}
& \mathcal{F}_{t}=\left\{\gamma \in \mathcal{P}^{r} \mid \sum_{j=1}^{i-1}(-1)^{j+1} \gamma_{j}=1+\min \left\{\sum_{j=i+1}^{2 i}(-1)^{j+1} h_{j}, \sum_{j=i+1}^{2 i}(-1)^{j+1} h_{j}\right\}\right\} \\
& \mathcal{F}_{b}=\left\{\gamma \in \mathcal{P}^{r} \mid \sum_{j=1}^{i-1}(-1)^{j+1} \gamma_{j}=1+\frac{1}{2} \sum_{j=0}^{2 i}(-1)^{j+1} h_{j}\right\} \\
& \mathcal{F}_{0}=\left\{\gamma^{r} \in \mathcal{P}^{r} \mid \gamma_{i-1}=0\right\} .
\end{aligned}
$$

Demonstração: $\Lambda$ matriz de coeficientes $A$ correspondente as desigualdades em (4.80), exceto as de não negatividade, é uma matriz $0, \pm 1$. É fácil ver que a matriz $0,1 \tilde{A}=\left(\left|a_{i j}\right|\right)$ tem propriedade de 1's consecutivos e assim é totalmente unimodular. Como $A$ é obtida de $\tilde{A}$ multiplicando as colunas pares por -1 , esta última matriz é também totalmente unimodular. Levando em conta o fato que o lado direito de (4.80) é inteiro, concluímos que todos os vértices em $\mathcal{P}^{r}$ são inteiros.

A correspondência entre pontos inteiros de $\mathcal{P}^{r}$ e $h^{c d}$ satisfazendo (4.34) imita a prova do item 3 da Proposição 4.2.1.

O argumento que justifica a última afirmação é similar ao dado na prova do item 2 da Proposição 4.2.1. Para que um $\bar{\gamma}^{r}$ em $\mathcal{P}^{r}$ seja um vértice, o conjunto de restrições saturadas em $\bar{\gamma}^{r}$ deve incluir uma das quatro desigualdades envolvendo $\gamma_{i-1}$ em (4.80), repetidas abaixo por conveniência. Note que usamos o fato que $i$ é par.

$$
\begin{aligned}
\sum_{j=1}^{i-1}(-1)^{j+1} \gamma_{j} & \leq 1+\sum_{j=i+1}^{2 i}(-1)^{j+1} h_{j} \\
\sum_{j=1}^{i-1}(-1)^{j+1} \gamma_{j} & \leq 1+\sum_{j=i+1}^{2 i}(-1)^{j+1} h_{j} \\
\sum_{j=1}^{i-1}(-1)^{j+1} \gamma_{j} & \geq 1+\frac{1}{2} \sum_{j=0}^{2 i}(-1)^{j+1} h_{j} \\
\gamma_{i-1} & \geq 0
\end{aligned}
$$

As desigualdades (4.81)-(4.82) são equivalentes às desigualdades abaixo.

$$
\sum_{j=1}^{i-1}(-1)^{j+1} \gamma_{j} \leq 1+\min \left\{\sum_{j=i+1}^{2 i}(-1)^{j+1} h_{j}, \sum_{j=i+1}^{2 i}(-1)^{j+1} h_{j}\right\} .
$$


Como uma das três desigualdades (4.85), (4.83) ou (4.84), deve estar saturada em um vértice, o vértice deve pertencer a $\mathcal{F}_{t}, \mathcal{F}_{b}$ ou $\mathcal{F}_{0}$, respectivamente.

A próxima proposição é a análoga à Proposição 4.2 .2 para o caso $n=0 \bmod 4$. Como antes, escolhemos o vetor $\breve{\gamma}$ correspondente à solução $\bar{h}^{c d}$ de (4.35) satisfazendo (4.41).

Proposição 4.2.4 Assuma que (4.35) tem uma solução não negativa. Seja $\tilde{\gamma}$ a solução inteira não negativa de (4.38)-(4.40) correspondendo a $\tilde{h}^{\text {cd }}$, a solução inteira não negativa de (4.35) que satisfaz (4.41). O politopo $\mathcal{P}^{r}$ pode ser reeescrito como

$\mathcal{P}^{r}=\left\{\begin{array}{l|}0 \leq \gamma^{r} \\ (-1)^{k+1} \sum_{j=0}^{k}(-1)^{j+1} \gamma_{j} \leq(-1)^{k+1} \sum_{j=0}^{k}(-1)^{j+1} \gamma_{j}, \quad \text { para } 1 \leq k \leq i-1 \\ \sum_{j=0}^{i-1}(-1)^{j+1} \gamma_{j} \geq \frac{1}{2} \sum_{j=0}^{2 i}(-1)^{j+1} h_{j}\end{array}\right\}$

Além disso, $\tilde{\gamma}$ (resp., $\tilde{\gamma}^{r}$ ) é um vértice e o vetor máximo em $\mathcal{P}\left(\right.$ resp. $\left.\mathcal{P}^{r}\right)$, componentea-componente.

Demonstração: Se assumimos que (4.35) tem uma solução, as desigualdades $0 \geq 1-$ $h_{2 i}$ e $0 \geq 1-h_{0}$ são redundantes e podem ser eliminadas. Agrupando o restante das desigualdades em (4.38) temos

$$
\begin{aligned}
\sum_{j=1}^{k}(-1)^{j+1} \gamma_{j}\left\{1+\min \left\{\sum_{j=2 i-k}^{2 i}(-1)^{j+1} h_{j}, \sum_{j=0}^{k}(-1)^{j+1} h_{j}\right\}\right. & \text { se } 1 \leq k \leq i-1, k \text { ímpar } \\
\geq 1+\max \left\{\sum_{j=2 i-k}^{2 i}(-1)^{j+1} h_{j}, \sum_{j=0}^{0}(-1)^{j+1} h_{j}\right\} & \text { se } 1 \leq k \leq i-1, k \text { par } \\
\sum_{j=1}^{i-1}(-1)^{j+1} \gamma_{j} \geq 1+\frac{1}{2} \sum_{j-0}^{2 i}(-1)^{j+1} h_{j} & \text { for } 1 \leq j \leq i-1
\end{aligned}
$$

Agora $\tilde{\gamma}$ satisfaz a primeira equação em (4.38) e (4.51). Usando a primeira equação 
em (4.38) para eliminar $\tilde{\gamma}_{i}$ em (4.51), e então usando (4.40), temos

$$
\begin{aligned}
& -\sum_{j=2 i+1-k}^{2 i}(-1)^{j+1} \tilde{\gamma}_{j}+(-1)^{2 i+1-k} h_{2 i-k}^{d} \\
= & -\sum_{j=0}^{k-1}(-1)^{j+1} \tilde{\gamma}_{j}+(-1)^{2 i+1-k} h_{2 i-k}^{d} \\
= & -\sum_{j=2 i+1-k}^{2 i}(-1)^{j+1} h_{j}, \quad \text { para } 1 \leq k \leq i
\end{aligned}
$$

A equação (4.54) pode ser reescrita como

$$
\sum_{j=0}^{k-1}(-1)^{j+1} \tilde{\gamma}_{j}+(-1)^{k} h_{k}^{c}=\sum_{j=0}^{k-1}(-1)^{j+1} h_{j}, \quad \text { para } 2 \leq k \leq i .
$$

As equações (4.88) (4.89), os fatos que $\tilde{h}^{\text {cd }}$ satisfaz (4.8), $\tilde{\gamma}_{0}=1$ e que $\tilde{\gamma}^{r}$ satisfaz (4.80) implicam

$$
\min \left\{\sum_{j=2 i-k}^{2 i}(-1)^{j+1} h_{j}, \sum_{j=0}^{k}(-1)^{j+1} h_{j}\right\}=\sum_{j=0}^{k}(-1)^{j+1} \tilde{y}_{j}, \quad \text { para } 1 \leq k \leq i-1, k \text { impar }
$$

$\mathrm{e}$

$$
\max \left\{\sum_{j=2 i-k}^{2 i}(-1)^{j+1} h_{j}, \sum_{j=0}^{0}(-1)^{j+1} h_{j}\right\}=\sum_{j-0}^{k}(-1)^{j+1} \tilde{\gamma}_{j}, \quad \text { para } 1 \leq k \leq i-1, k \text { par. }
$$

Substituindo as expressões acima em (4.87) obtemos (4.86).

Com respeito da última afirmação da proposição, note que a primeira desigualdade em (4.87) lê-se $\gamma_{1}<\tilde{\gamma}_{1}$ e as desigualdades correspondentes a $k=\ell-1$ e $k=\ell$ implicam $\gamma_{\ell} \leq \check{\gamma}_{\ell}$, para $2 \leq \ell_{1}-1$. Portanto $\tilde{\gamma}^{r}$ é o vetor máximo, componente-a-componente, de $\mathcal{P}^{r}$. Isto implica que ele é um vértice de $\mathcal{P}^{r}$. Finalmente, (4.79) e (4.86) implicam, usando a fato que $i$ é par,

$$
\gamma_{i}=2\left(\sum_{j=0}^{i-1}(-1)^{j+1} \gamma_{j}-\sum_{j=0}^{2 i}(-1)^{j+1} h_{j}\right) \leq 2\left(\sum_{j=0}^{i-1}(-1)^{j+1} \tilde{\gamma}_{j}-\sum_{j=0}^{2 i}(-1)^{j+1} h_{j}\right),
$$

que dá um limite superior para $\gamma_{i}$, realizado por $\tilde{\gamma}_{i}$. Assim $\tilde{\gamma}$ é o vetor máximo em $\mathcal{P}$, componente-a-componente, e portanto um vértice de $\mathcal{P}$.

O próximo exemplo ilustra a relação entre as soluçōes inteiras nāo negativas $h^{c d}$ de (4.35) e os vetores inteiros $\gamma^{r}$ de $\mathcal{P}^{r}$. 
Exemplo Seja $n=2 i=8$ e $\left(h_{0}, \ldots, h_{8}\right)=(3,5,7,8,5,2,2,2,2)$. A Figura 4.5 dá uma representação não ortodoxa para as restriçōes (4.35). Note que os dois arcos pendurados nos dois nós mais à direita representam a mesma variável, a saber $h_{4}^{d}$. Isto não está de acordo com as convençōes assumidas para problemas de flucos em redes. Assim, enquanto $h^{c d 1}$ e $h^{c d 2}$, estão associados aos ciclos 1 e 2 como no último exemplo, existe uma terceira "circulação" $h^{\text {cd } 3}$ cujo suporte é dado por $\left(h_{4}^{c}, \beta, h_{4}^{d}\right)=(1,-2,1)$. No entanto, se levarmos esta anomalia em consideração, podemos ainda usar a figura para rapidamente calcular todas as possíveis soluções inteiras, quc podem ser obtidas acrescentando múltiplos inteiros de $h^{c d i}$, para $1 \leq i \leq 3$, à soluçāo $\tilde{h}^{c d}$ satisfazendo (4.41) descrita na Figura 4.5.

$$
\begin{aligned}
\gamma_{1} & \leq 1 \\
\gamma_{1}-\gamma_{2} & \geq-1 \\
\gamma_{1}-\gamma_{2}+\gamma_{3} & \leq 1 \\
\gamma_{1}-\gamma_{2}+\gamma_{3} & \geq 0 \\
\gamma_{1}, \gamma_{2}, \gamma_{3} & \geq 0
\end{aligned}
$$

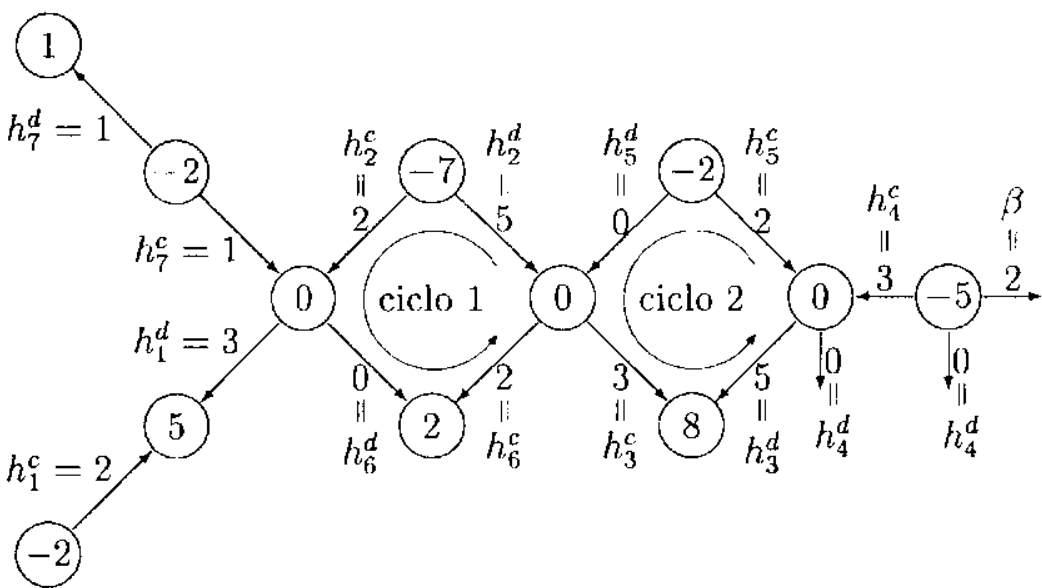

Figura 4.5: Solução $\tilde{h}^{c d}$ do exemplo.

O Politopo $\mathcal{P}^{r}$ correspondente a um determinadao dado é mostrado na Figura 4.6. Todos os inteiros $\gamma^{r}$ podem ser obtidos começando no vetor máximo $\tilde{\gamma}^{r}$ e adicionando múltiplos inteiros apropriados dos vetores $(-1,-1,0),(0,-1,-1),(0,0,-1),(1,0,-1) \mathrm{e}$ 
$(-1,0,0)$. A operação correspondente sobre $h^{\text {cd }}$ é mostrada abaixo.

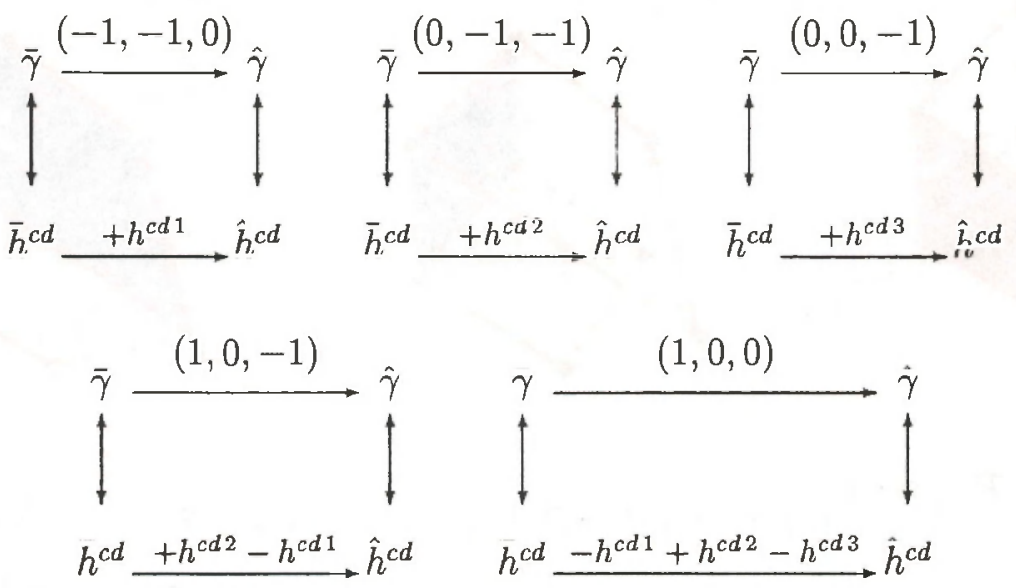

\subsubsection{Caso $n=2 \bmod 4$}

Suponha $n=2 i$, onde $i \geq 3$ é ímpar, e $\sum_{j=o}^{i \cdot i}(-1)^{j+1} h_{j}$ è par. As restrições (4.64) são uma cópia de (4.38) e pode ser manipulada como na seção 4.2 .2 para obter o sistema equivalente menor (4.80) em $\gamma^{r}=\left(\gamma_{1}, \ldots, \gamma_{i-1}\right)$. As Proposições 4.2 .3 e 4.2 .4 assim também se mantêm para o caso $n=2 \bmod 4$, com a regra para a construção de $h^{\text {:d }}$ ligeiramente diferente correspondendo a um dado $\gamma$ satisfazendo (4.64) dado no final da prova da Proposição 4.1.7. 

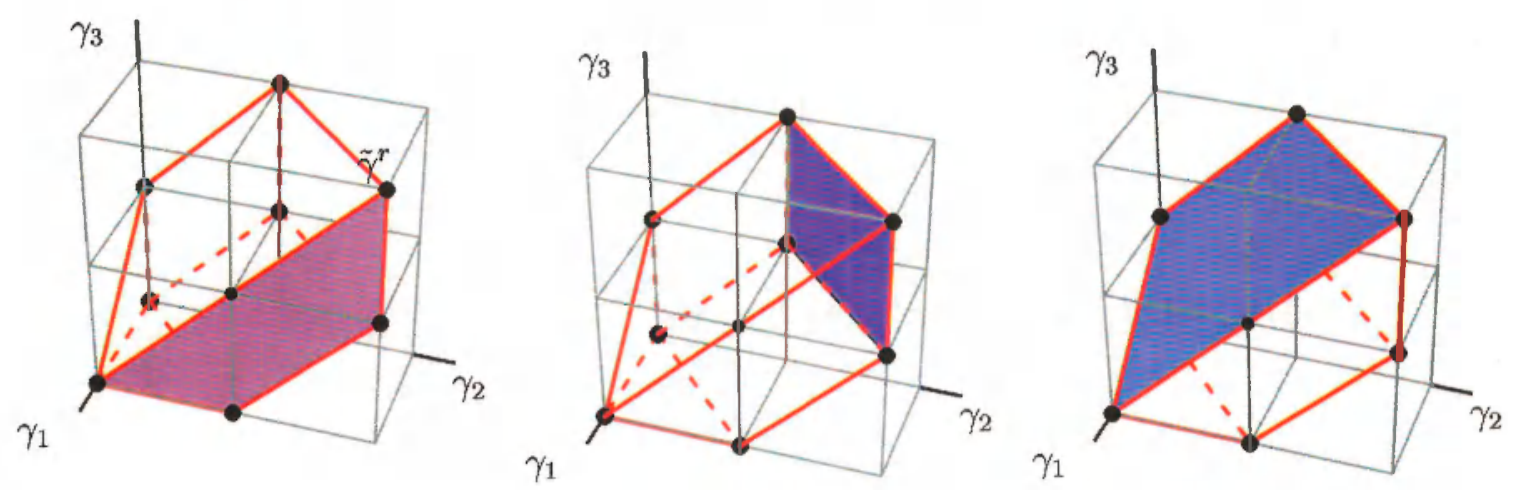

(a) $\mathcal{F}^{1}=\left\{\gamma^{r} \in \mathcal{P}^{r} \mid \gamma_{1}=\right.$ $1\}$.

(b) $\mathcal{F}^{2}=\left\{\gamma^{r} \in \mathcal{P}^{r} \mid \gamma_{1}-\gamma_{2}=-1\right\}\left(\right.$ c) $\mathcal{F}^{3}=\mathcal{F}_{t}=\left\{\gamma^{r} \in \mathcal{P}^{r} \mid\right.$ $\left.\gamma_{1}-\gamma_{2}+\gamma_{3}=1\right\}$.
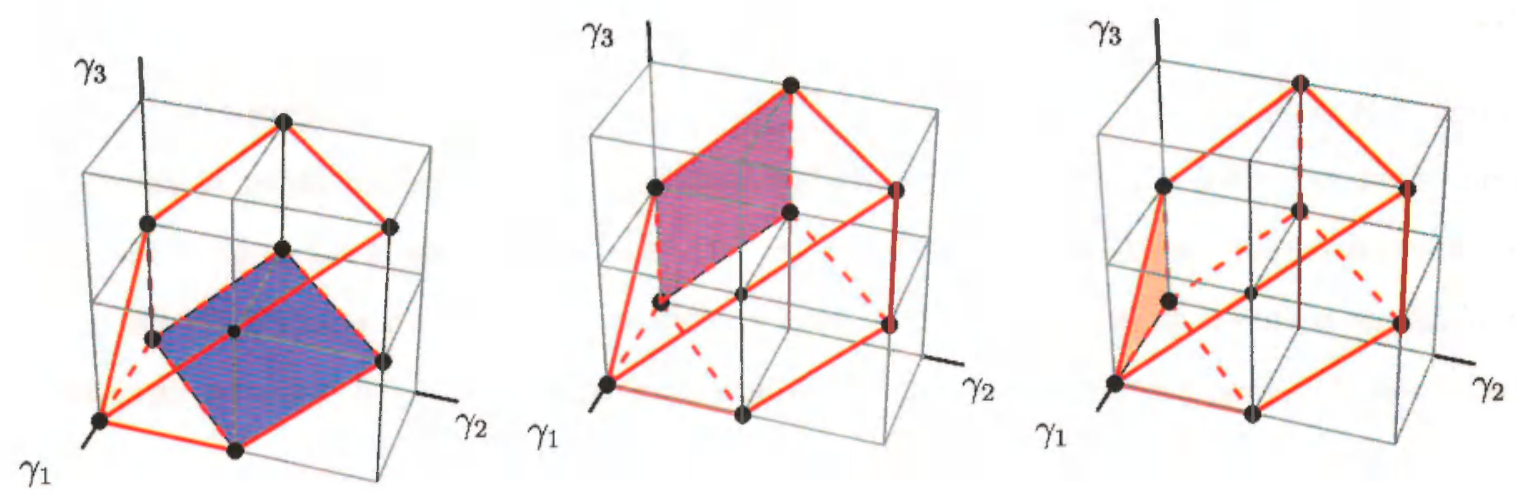

(d) $\mathcal{F}^{4}=\mathcal{F}_{b}=\left\{\gamma^{r} \in \mathcal{P}^{r}\right.$ $\left.\gamma_{1}-\gamma_{2}+\gamma_{3}=0\right\}$.

(e) $\mathcal{F}^{5}=\left\{\gamma^{r} \in \mathcal{P}^{r} \mid \gamma_{1}=\right.$

(f) $\mathcal{F}^{6}=\left\{\gamma^{r} \in \mathcal{P}^{r} \mid \gamma_{2}=\right.$ $0\}$.

$0\}$.

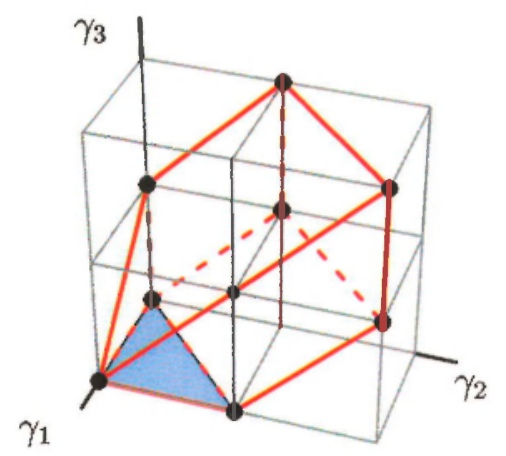

(g) $\mathcal{F}^{7}=\mathcal{F}_{0}=\left\{\gamma^{r} \in \mathcal{P}^{r} \mid \gamma_{3}=0\right\}$.

Figura 4.6: Faces de $\mathcal{P}^{r}$ e grade inteira. 


\section{Capítulo 5}

\section{Grafos de Lyapunov, Desigualdades de Poincaré-Hopf e Desigualdades de}

\section{Morse}

Neste capítulo trataremos dos grafos de Lyapunov em toda sua generalidade, isto é, consideraremos grafos em dimensão maior ou igual a dois com cycle number $\kappa$. Estaremos generalizando os resultados dos Capítulos 3 e 4 . Mais precisamente mostraremos os seguintes dois resultados que generaliza o Teorema de Continuação demonstrado no Capítulo 3.

Teorema 5.0.5 Considere um fluxo sobre um bloco isolante $B$ e seu semi-grafo de Lyapunov $L_{B}$ onde o vértice $v$ associado a $B$ é rotulado com $\left(h_{0}(v), \ldots, h_{n}(v), \kappa_{v}\right)$ então $L_{B}$ admite continuações a semi-grafos do tipo Morse com cycle rank maior ou igual a $\kappa_{v}$ se e somente se, $L_{B}$ satisfaz as desigualdades de Poincaré-Hopf (2.20)-(2.16). Mais ainda, $\kappa_{v} \leq \min \left\{h_{1}-\left(e^{*}+h_{0}-1\right), h_{n-1}-\left(e^{+}+h_{n}-1\right)\right\}$.

O Teorema 5.0.5 implica no seguinte teorema.

Teorema 5.0.6 Todo grafo abstrato de Lyapunov $L\left(h_{0}, \ldots, h_{n}, \kappa\right)$ que satisfaz as desigualdades de Poincaré-Hopf (2.20) (2.16) em cada vértice pode ser continuado a um grafo abstrato de Lyapunou do tipo Morse com cycle rank maior ou igual a $\kappa$. Mais ainda, $\kappa \leq \min \left\{h_{1}-\left(h_{0}-1\right), h_{n-1}-\left(h_{n}-1\right)\right\}$. 
No Capítulo 4 foi mostrado que o caso particular das desigualdades de Poincaré-Hopf (2.21)-(2.23) com $\kappa=0$ são condições necessárias e suficientes para as desigualdades de Morse generalizadas (1) se manterem para algum vetor de números de Betti. Neste capítulo mostraremos que:

Teorema 5.0.7 Um grafo abstrato Lyapunov $L\left(h_{0}, \ldots, h_{n}, \kappa\right)$ satisfaz as desigualdades de Poincaré-Hopf (2.21)-(2.23) se, e somente se, $L\left(h_{0}, \ldots, h_{n}, \kappa\right)$ satisfaz as desigualdades de Morse (1) e a desigualdade $\gamma_{1} \geq \kappa$ para algum vetor de números de Betti $\left(\gamma_{0}, \ldots, \gamma_{n}\right)$.

Também neste capítulo estabeleceremos que as desigualdades (1), $\gamma_{1} \geq \kappa$ e as restrições que um vetor de números de Betti devem satisfazer, ou seja, $\gamma_{0}=\gamma_{n}=1, \gamma_{j}=\gamma_{n-j}$, para $j=1, \ldots, n-1, \gamma \geq 0$, definem um politopo inteiro, como no capítulo anterior. Mais precisamente mostraremos o seguinte resultado.

Teorema 5.0.8 Um grafo $L\left(h_{0}, \ldots, h_{n}, \kappa\right)$ que satisfaz as desigualdades de Poincaré-Hopf determina o politopo de Morse $\mathcal{P}_{\kappa}\left(h_{0}, \ldots, h_{n}\right)$. Mais ainda, temos $\mathcal{P}_{\kappa_{1}}\left(h_{0}, \ldots, h_{n}\right) \subset$ $\mathcal{P}_{\kappa_{2}}\left(h_{0}, \ldots, h_{n}\right)$, para $\kappa_{1} \geq \kappa_{2}$.

Estes resultados generalizam resultados análogos obtidos no Capítulo 4 para o caso $\kappa=0$. Note que se $\kappa=0, \mathcal{P}_{\kappa}\left(h_{0}, \ldots, h_{n}\right)=\mathcal{P}\left(h_{0}, \ldots, h_{n}\right)$.

Este capítulo está dividido nas seguintes seçoes. Na Seção 5.1, mostraremos que o sistema $h_{\kappa}^{c d}$ tem uma solução se, e somente se, as desigualdades de Poincaré-Hopf para blocos isolantes estão satisfeitas. Logo, provaremos um teorema de continuação para grafos abstratos de Lyapunov com a presença de ciclos. Na Seção 5.2 apresentaremos o sistema reduzido $h_{\kappa}^{c d}$ que tem uma solução se, e somente se, as desigualdades de Poincaré-Hopf para variedades fechadas são satisfeitas. Usamos as soluçōes do sistema reduzido $h_{\kappa}^{\text {cd }}$ para estabelecer a suficiência das desigualdades de Poincaré-Hopf para que as desigualdades de Morse sejam satisfeitas e por último, provamos a necessidade. Na Seção 5.3 descrevemos o politopo de Morse e provamos o Teorema 5.0.8. Também, propriedades geométricas adicionais do politopo serão apresentadas. 


\subsection{Resultados de Continuação}

A prova do Teorema 5.0.5 é dividida nas duas seguintes proposições. No Capítulo 3 foi demonstrada a seguinte proposição $\operatorname{com} \kappa=0$.

Proposição 5.1.1 Um vértice do tipo sela v rotulado com $\left(0, h_{1}, \ldots, h_{n-1}, 0, \kappa\right)$ pode ser explodido a um semi-grafo de Lyapunov do tipo Morse com cycle rank maior ou igual a $\kappa$ e $\kappa \leq \min \left\{h_{1}-\left(e^{-}-1\right), h_{n-1}-\left(e^{+}-1\right)\right\}$, se, e somente se, o sistema $h_{\kappa}^{c d}$ (4.1) ou (4.2) tem uma solução inteira não negativa $\left(h_{1}^{c}, h_{1}^{d}, \ldots, h_{n-1}^{c}, h_{n \cdot 1}^{d}\right)$.

Demonstração: Segue diretamente dos passos descritos no algoritmo de explosão do vértice tipo sela para a construção dos sistemas that $h_{\kappa}^{c d}$.

No Capítulo 3 consideramos as seguintes formas gerais dos sistemas lineares (4.1) e (4.2):

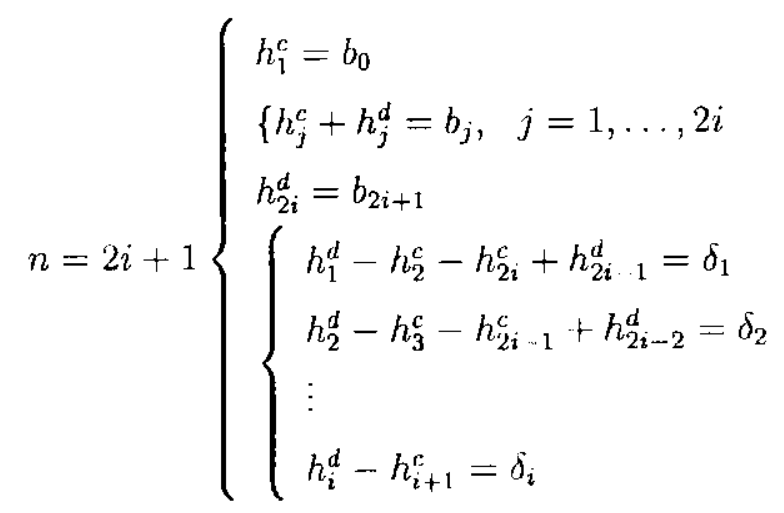

oll

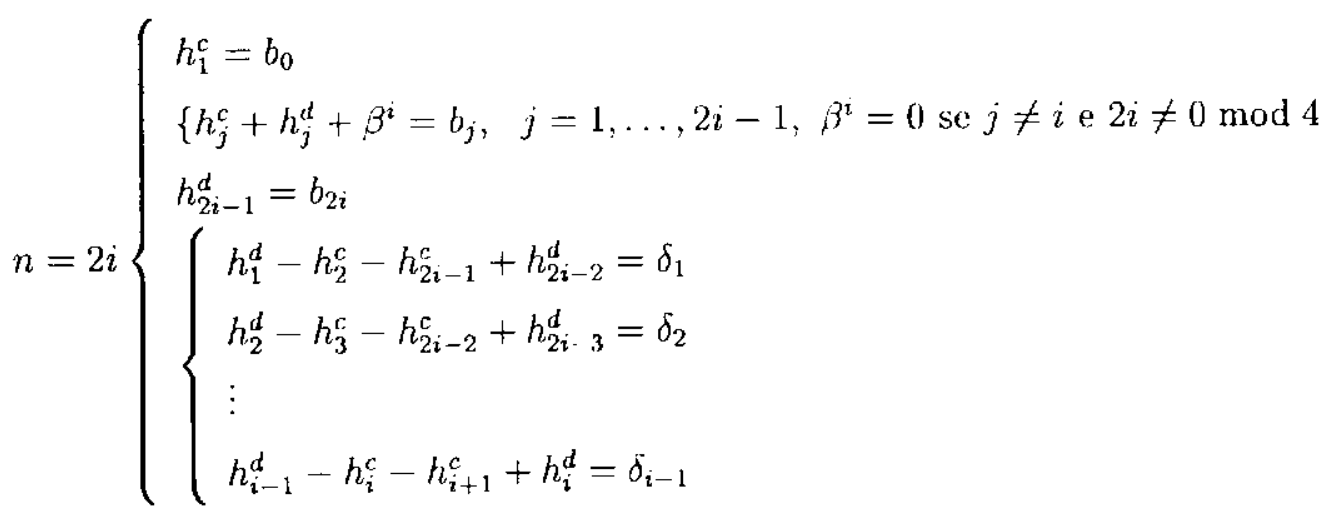


Os casos especiais (4.1) e (4.2) podem ser obtidos com as seguintes substituições:

$$
\begin{aligned}
& n=2 i+1\left\{\begin{aligned}
b_{0} & =-1+e+\kappa \\
b_{j} & =h_{j}, \quad \text { para } j=1, \ldots, 2 i \\
b_{2 i+1} & =-1+e^{+}+\kappa \\
\delta_{j} & =B_{j}^{+}-B_{j}^{-}, \quad \text { para } j=1, \ldots, i-1 \\
\delta_{i} & =\left(B_{i}^{+}-B_{i}^{-}\right) / 2
\end{aligned}\right. \\
& n=2 i\left\{\begin{aligned}
b_{0} & =-1+e^{-}+\kappa \\
b_{j} & =h_{j}, \quad \text { for } j=1, \ldots, 2 i-1 \\
b_{2 i} & =-1+e^{+}+\kappa \\
\delta_{j} & =B_{j}^{+}-B_{j}^{-}, \quad \text { para } j=1, \ldots, i-1 .
\end{aligned}\right.
\end{aligned}
$$

Foi também mostrado no Capítulo 3 que o sistema (5.1) pode ser visto como um problema de fluxo em rede por meio de uma mudança adequada de sinais em metade das equações. As variáveis $h^{\text {cd }}$ são interpretadas como fluxos sobre os arcos da rede e cada equação pode ser lida como "fluxo que entra - fluxo que sai = constante (demanda/suprimento) associada ao nó". As redes correspondentes a $n=2 i+1$ são descritas nas Figuras 5.1 e 5.2, com as constantes associadas aos nós indicadas dentro dos nós. $\mathrm{Na}$ imersão planar adotada nesta figura, a forma em zig-zag da componente digrafo da rede forma uma seqüência de losangos com um losango "aberto" em uma das extremidades. Arcos correspondentes as variáveis $\left(h_{1}^{c}, h_{1}^{d}, h_{2}^{c}, h_{2}^{d}, \ldots, h_{2 i}^{c}, h_{2 i}^{d}\right)$, nesta ordem, formam um caminho não orientado Euleriano cobrindo todo o digrafo.

Quando $n=2 i+1$ e $i$ é par, o digrafo é um pouco diferente, ver o ciclo mais à direita no digrafo da Figura 5.2. Ambos os digrafos contêm uma cadeia de $i-1$ ciclos de comprimento quatro e a seqüência de arcos associadas com $\left(h_{1}^{c}, h_{1}^{d}, h_{2 i}^{c}, h_{2 i}^{d}\right)$ formam um caminho não orientado que é adjacente ao primeiro ciclo. Os arcos no $j$-ésimo ciclo estão associados com as variáveis $h_{j+1}^{d}, h_{2 i-j}^{c}, h_{2 i-j}^{d}$ e $h_{j+1}^{c}$, e a orientação dos dois primeiros arcos é oposta a orientação dos dois últimos, com respeito a uma orientação arbitrária do ciclo.

O conjunto das equações (5.2) pode ser visto como um problema de fluxo em rede com restrições adicionais. A Figura 5.3 representa a "rede" para o caso par $n=0$ mod 4 . As aspas são necessárias pelo fato de que este problema não pode ser classificado como 


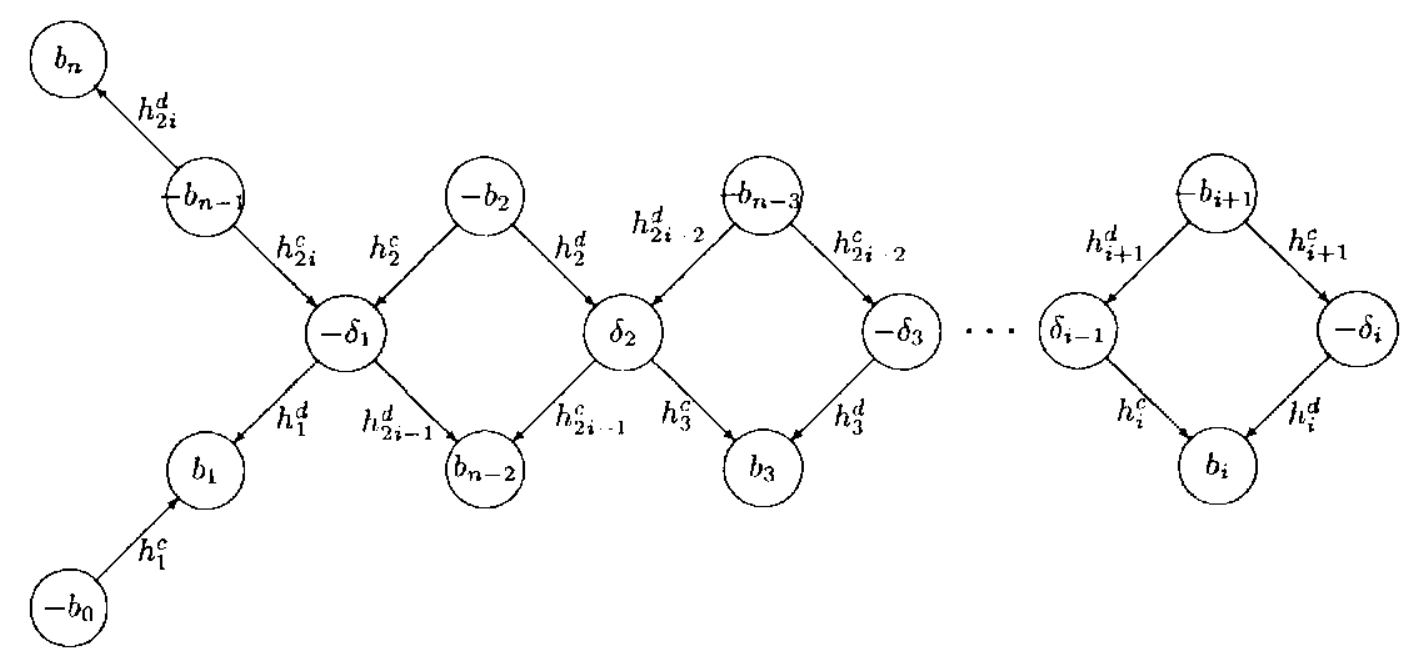

Figura 5.1: Rede para o caso $n=2 i+1, i$ ímpar.

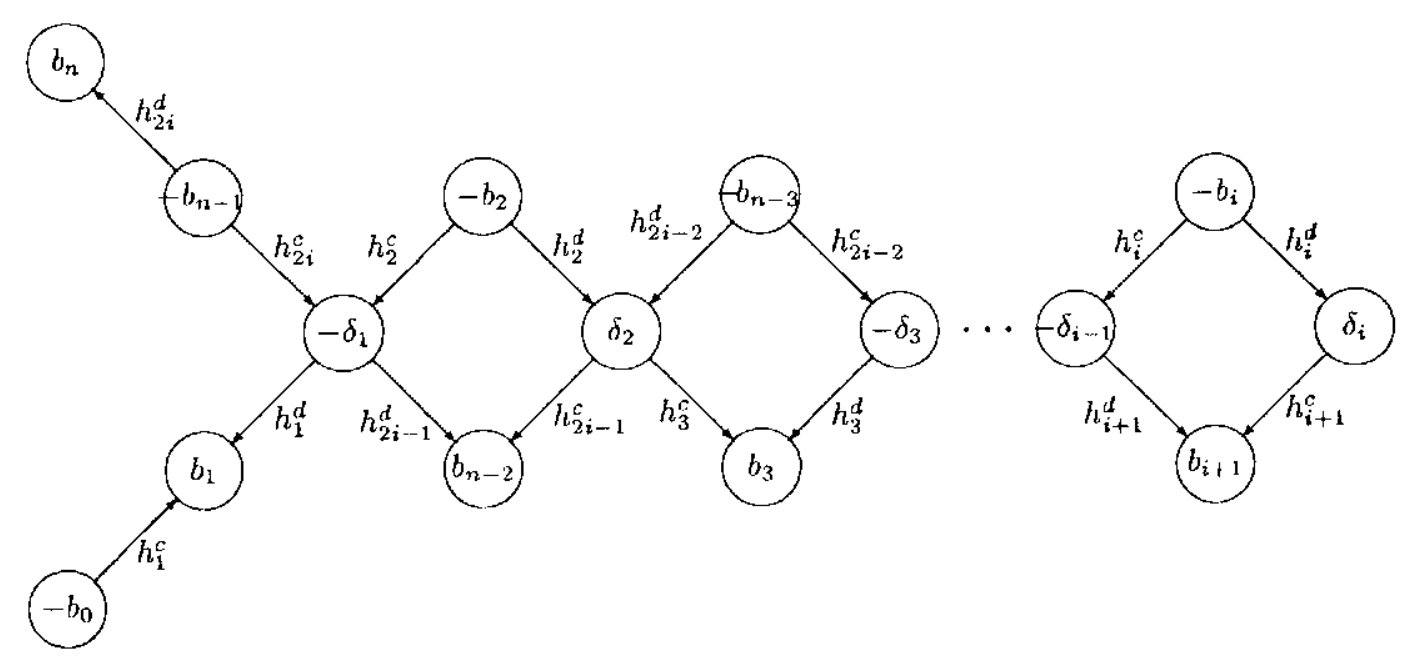

Figura 5.2: Rede para $n=2 i+1, i$ par. 
um problema clássico de fluxo em rede. Note na figura os arcos verticais apontando para baixo na direita. Estes arcos correspondem à mesma variável no sistema linear, o que não é permitido no modelo clássico. Poderíamos associar a cada arco uma variável diferente, digamos $\tilde{h}_{i}^{d}$ e hath $h_{i}^{d}$, e acrescentar a restrição adicional $\tilde{h}_{i}^{d}=h a t h_{i}^{d}$, criando assim um problema de fluxo com restriçōes adicionais. O fato que os arcos estão "pendurados", ou sejam, não terminam em nós, não é problema: pode-se criar um nó que reccba todos estes arcos e colocar como constante para este nó o negativo da soma das constantes de todos os outros nós, pois a equação associada a este nó seria redundante. Note, no entanto, que, se fixamos os valores de $h_{i}^{d}$ obtemos um problema de fluxo em rede usual.

A Figura 5.4 descreve a "rede" correspondente ao caso $n=2 \bmod 4$. Novamente o problema não é um problema clássico de fluxo em rede, as considerações feitas no parágrafo anterior podem ser aplicadas a este caso também. Ambos os problemas são suficientemente próximos do problema clássico, sendo fácil realizar as adaptações necessárias à teoria geral e aos métodos para pode empregá-los na resolução deste caso especial. Portanto, para simplificar a discussão, iremos nos referir a todos estes problemas como problemas de fluxo em rede, chamando atenção para as considerações especiais que os casos pares requerem quando aparecem.

A solução geral do sistema (5.1) (ou (5.2)) é a soma de uma solução particular e uma solução da versão homogênea do sistema, isto é, uma solução que satisfaz a condição "fluxo que entra = fluxo que sai"em todo nó. $\Lambda$ primeira é chamada de fluxo e, a última, de circulação. Assim o conjunto de circulações é um subespaço linear, o espaço nulo da matriz dos coeficientes do sistema linear. Rockafellar em [20] mostrou que um vetor de um espaço linear é a soma de vetores elementares deste espaço. Um vetor elementar de um subespaço é um vetor deste subespaço com suporte mínimo. É bem conhecido que as circulações elementares são aquelas cujo suporte corresponde a um ciclo simples no digrafo.

Portanto as soluções elementares de (5.1) e (5.2) são fáceis de determinar. Nos casos ímpar e par existern uma circulaçăo elementar associada a cada ciclo. Nos casos pares existe uma circulação elementar adicional, associada com aos fluxos na extremidade direita dos digrafos descritos nas Figuras 5.3 e 5.4. No caso $n=2 i=0 \bmod 4$ (resp., $n=2 i=$ $2 \bmod 4)$, esta circulação adicional tem $\left(h_{i}^{c}, \beta^{i}, h_{i}^{d}\right)=(1,-2,1)\left(\operatorname{resp} .,\left(h_{i}^{c}, h_{i}^{d}\right)=(11)\right)$ e 


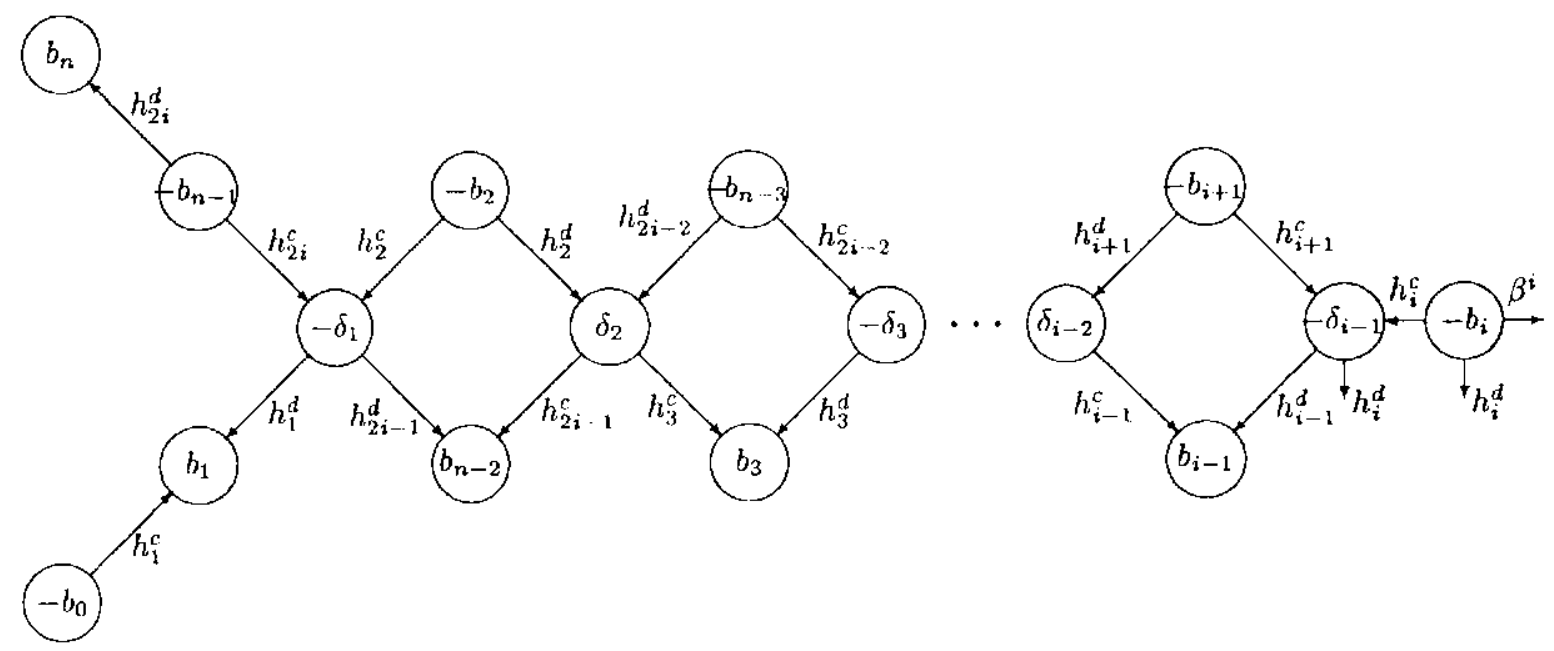

Figura 5.3: Rede para o caso $n=2 i=0 \bmod 4$.

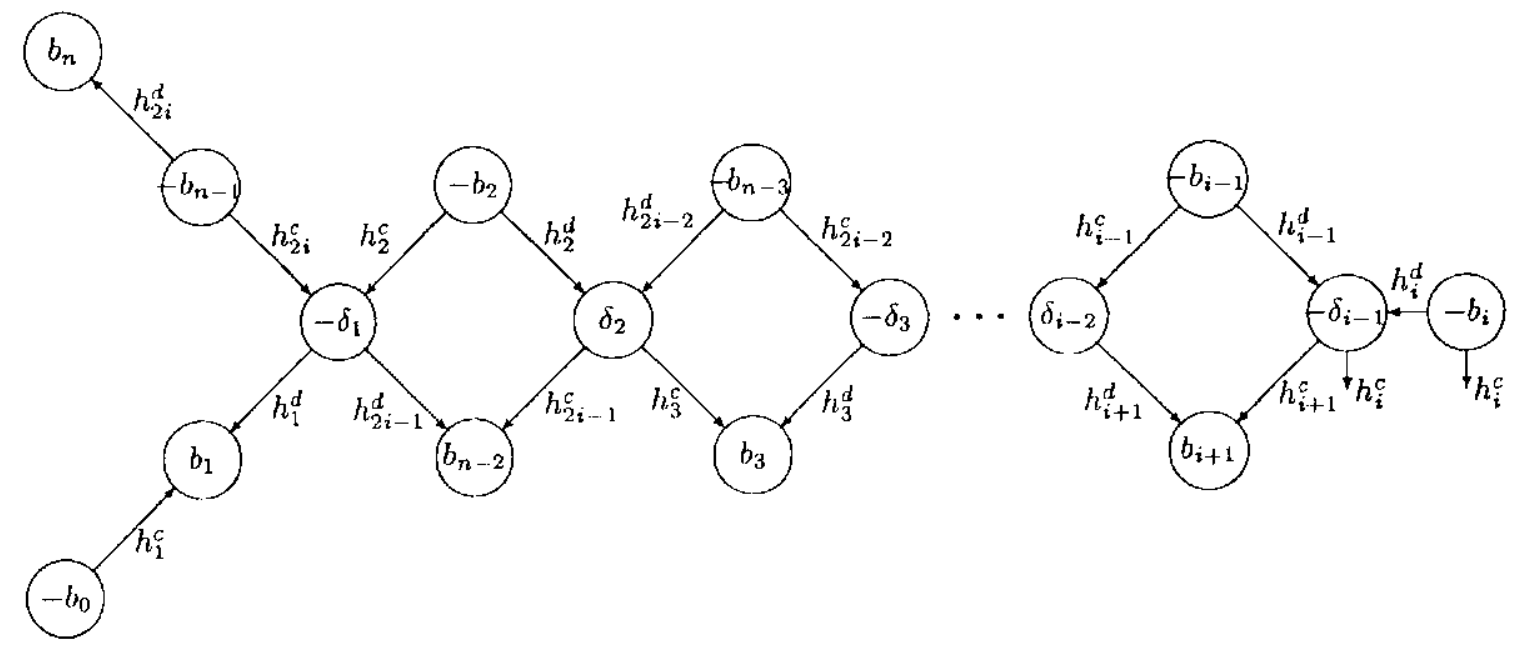

Figura 5.4: Rede para o caso $n=2 i=2 \bmod 4$. 
componentes restantes iguais a zero. Finalmente, se removermos um arco de cada ciclo e o arco associado a $h_{i}^{d}\left(\right.$ resp., $\left.h_{i}^{c}\right)$ no caso $n=2 i=0 \bmod 4$ (resp., $\left.n=2 i=2 \bmod 4\right)$ ), os arcos restantes formam uma árvore. As colunas da matriz de coeficientes do sisterna $h_{\kappa}^{c d}$ associadas ans arcos desta árvore são linearmente independentes e assim este subsistema tem uma solução única, se admitir solução (a submatriz do subsistema tem mais linhas do que colunas). Uma solução de um sistema linear cujo suporte corresponde a colunas linearmente independentes da matriz de coeficientes do sistema é chamada uma solução básica. Geometricamente, estas soluçöes básicas correspondem a pontos extremos, ou vértices, do poliedro definido pelas equaçōes de equilíbrio de fluxo e restrições de não negatividade. Pode-se também mostrar que cada vértice está associado a alguma árvore. Soluções básicas do sistema (5.1) (ou (5.2)) correspondentes a alguma árvore conhecida são fáceis de calcular: comece nas folhas da árvore (nós com apenas um arco incidente) e determine os fluxos dos arcos incidentes a estes nós; feito isso retire estes nós e arcos da árvore, modificando adequadamente as constantes dos nós adjacentes aos arcos que foram retirados e repita o processo até a retirada de todos os arcos. Em linguagem algébrica, isto é equivalente a permutar as linhas e colunas da submatriz associada ao problema de fluxo na árvore de modo a torná-la triangular e então resolver o sistema linear correspondente por substituição.

Proposição 5.1.2 Os sistemas $h_{\kappa}^{c d}(4.1)$ e (1.2) têm soluções inteiras não negativas se, $e$ somente se, as desigualdades de Poincaré-Hopf (2.20), (2.7) e (2.16), para blocos isolantes estão satisfeitas. Mais ainda, o conjunto de todas as soluçöes para o sistema $h_{\kappa}^{\text {cd }}$ podem ser obtidas de uma única solução básica e das circulações elementares do problema de fluxo em redes correspondente.

Demonstração: O truque para mostrar a equivalência entre os problemas de fluxo em rede e as desigualdades de Poincaré-Hopf no Capítulo 3 foi dividir o problema de fluxo em rede em um conjunto de $i$, no caso $n$ impar, ou $i+1$, no outro caso, problemas independentes menores de fluxo de rede. O primeiro passo tomado foi dividir o problema em dois. Isto foi feito eliminando do sistema as variáveis $h_{1}^{c}, h_{1}^{d}, h_{n-1}^{c}$ e $h_{n-1}^{d}$, cujos valores são facilmente determinados do sistema. Em termos de rede, isto é equivalente a dividir a rede em duas, como descrito na Figura 5.5. 


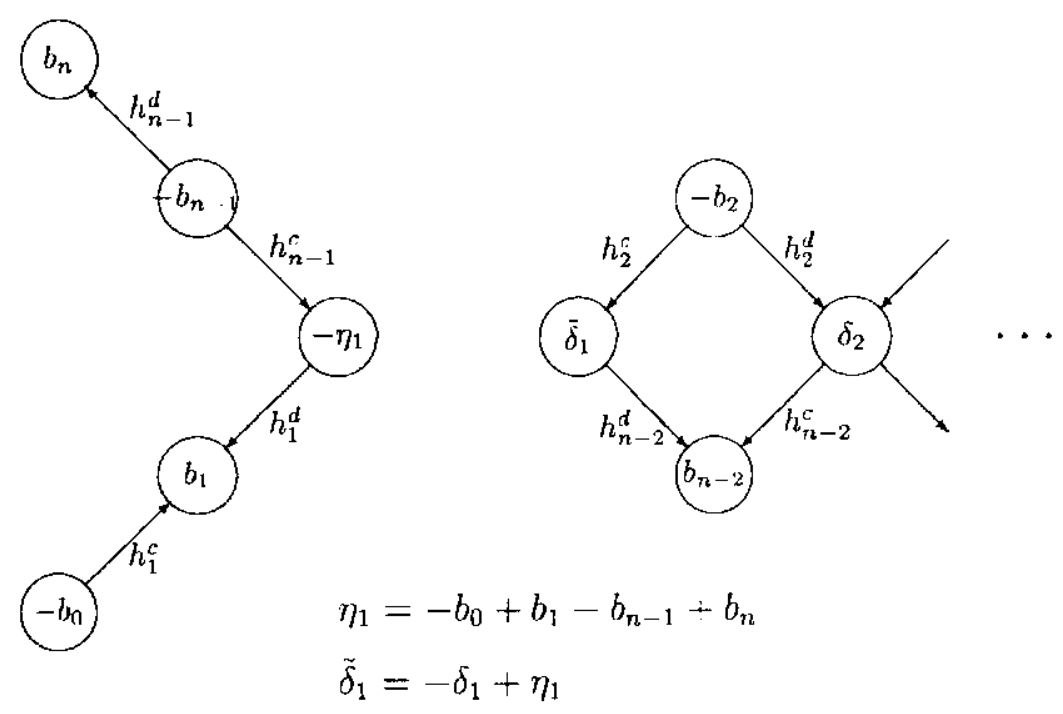

Figura 5.j̃: Dividindo o problema em dois.

Quatro variáveis em (5.1), ou (5.2), são determinadas de forma única:

$$
\left\{\begin{array} { r l } 
{ - h _ { 1 } ^ { c } } & { = - b _ { 0 } } \\
{ h _ { 1 } ^ { c } + h _ { 1 } ^ { d } } & { = b _ { 1 } } \\
{ - h _ { n - 1 } ^ { c } - h _ { n - 1 } ^ { d } } & { = - b _ { n - 1 } } \\
{ h _ { n - 1 } ^ { d } } & { = b _ { n } }
\end{array} \Rightarrow \left\{\begin{array}{rl}
h_{1}^{c} & =b_{0} \\
h_{1}^{d} & =b_{1}-b_{0} \\
h_{n-1}^{d} & =b_{n} \\
h_{n-1}^{c} & =b_{n-1}-b_{n}
\end{array}\right.\right.
$$

A rede da esquerda da Figura 5.5 contém as quatro equaçōes de (5.1), ou (5.2), mostradas acima, mais a equação redundante (por definição de $\eta_{1}$ ) $h_{n-1}^{c}-h_{1}^{d}=-\eta_{1}$. Substituindo os valores determinados acima na (única) equação de (5.1), ou (5.2), que contém as quatro variáveis $h_{1}^{d}, h_{2}^{c}, h_{n-1}^{c}$ e $h_{n-2}^{d}$, obtemos

$$
\begin{aligned}
h_{1}^{d}-h_{2}^{c}-h_{n-1}^{c}+h_{n-2}^{d} & =\delta_{1} \\
& \Downarrow \\
h_{2}^{c}-h_{n-2}^{d} & =-\delta_{1}+\eta_{1},
\end{aligned}
$$

que é precisamente a equação associada ao nó da extremidade esquerda da rede na direita da Figura 5.5. Como o resto da rede é uma cópia da rede original, todas as equações restantes estāo representadas nesta rede. Portanto o problema de fluxo de rede original foi dividido com sucesso em dois problemas de fluxo de rede independentes.

A mesma divisão pode ser feita em cada um dos nós na linha intermediária da rede, os nós com grau 4. A Figura 5.6 fornece as próximas três divisōes. Note que $\tilde{\delta}_{j}$ dá a 
soma cumulativa das constantes associadas aos nós à esquerda do nó com constante $\pm \delta_{j}$ inclusive.

$$
\tilde{\delta}_{j}=\sum_{k=0}^{j}\left((-1)^{k+1} b_{k}+(-1)^{k} b_{n-k}\right)+\sum_{k-1}^{j}(-1)^{k} \delta_{k}, \quad \text { para } j=1, \ldots, i-1 .
$$

Assim todos as constantes dos nós das redes independentes produzidos pelas divisões podem ser expressas em termos das constantes originais.
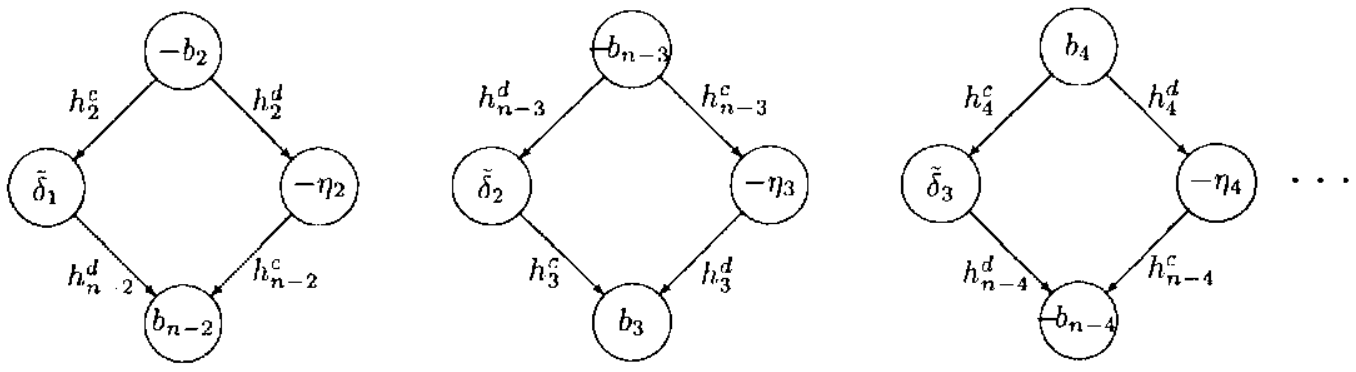

$$
\begin{aligned}
& \eta_{1}=-b_{0}+b_{1}-b_{n-1}+b_{n} \\
& \tilde{\delta}_{1}=-\delta_{1}+\eta_{1} \\
& \eta_{2}=\tilde{\delta}_{1}-b_{2}+b_{n-2}
\end{aligned}
$$$$
\tilde{\delta}_{2}=\delta_{2}+\eta_{2}
$$$$
\tilde{\delta}_{3}=-\delta_{3}+\eta_{3}
$$$$
\eta_{3}=\tilde{\delta}_{2}+b_{3}-b_{n-2}
$$$$
\eta_{4}=\tilde{\delta}_{3}+b_{4}-b_{n-4}
$$

Figura 5.6: Divisão seguinte.

A Figura 5.7 fornece a última divisão para todos os casos. Note que a divisão de redes correspondentes ao caso $n=2 i+1$ produz apenas dois tipos de redes: a rede "caminho" na esquerda da Figura 5.5 e a rede "ciclo", cuja componente do digrafo é um ciclo não orientado de comprimento 4 . Um representante típico desta última rede é ilustrado na Figura 5.8. Por outro lado, a divisão de redes correspondentes ao caso $n=2 i$ produzem três tipos de redes, dois deles coincidem com os obtidos no primeiro caso, mas o último não é em geral uma rede pois contém os problemáticos arcos "pendurados", associados ao mesmo fluxo, ver Figura 5.7.

O problema de determinar se (5.1) (ou (5.2)) tem uma solução inteira não negativa se reduz a verificar se cada um dos sistemas lineares independentes produzidos com a divisão tem tal solução. Isto segue diretamente, dada a estrutura simples dos sistemas lineares independentes.

O primeiro sistema linear, correspondendo à rede caminho, é o mais fácil, pois tem solução única. Levando em conta que $b$ e $\delta$ são vetores inteiros não negativos, a única 

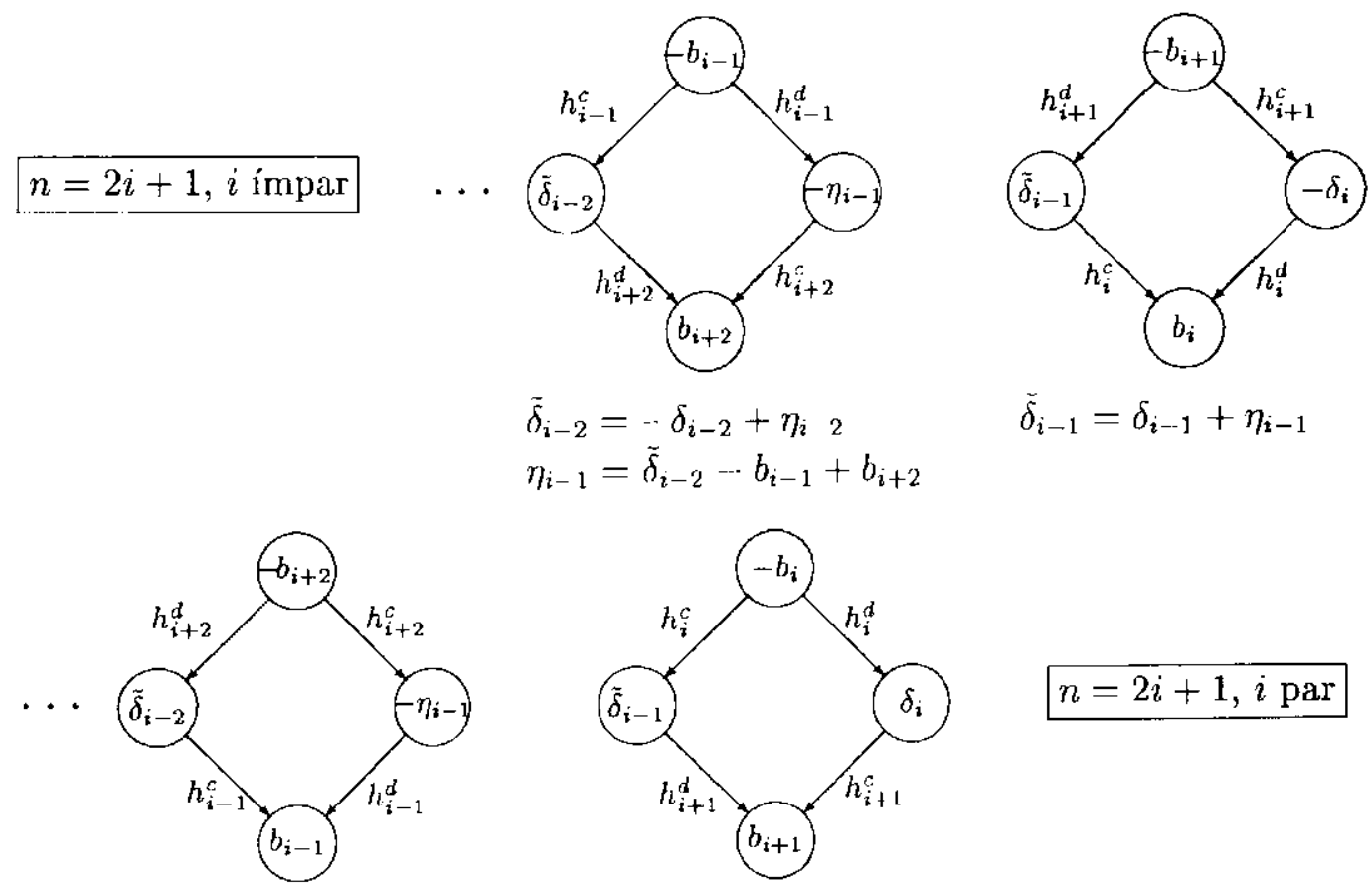

$n=\overline{2 i}+1, i \mathrm{par}$

$\bar{\delta}_{i-2}=\delta_{i-2}+\eta_{i-2}$

$\tilde{\delta}_{i-1}=-\delta_{i-1}+\eta_{i-1}$

$\eta_{i-1}=\tilde{\delta}_{i-2}-b_{i-1}+b_{i+2}$

$n=2 i=0 \bmod 4$
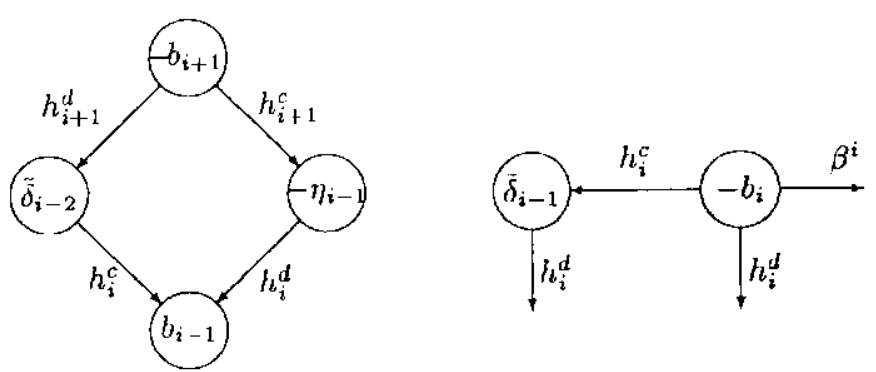

$$
\begin{aligned}
& \tilde{\delta}_{i-2}=\delta_{i-2}+\eta_{i-2} \\
& \eta_{i-1}=\tilde{\delta}_{i \cdot 2}-b_{i+1}+b_{i-1}
\end{aligned}
$$$$
\bar{\delta}_{i-1}=-\delta_{i-1}+\eta_{i} 1
$$
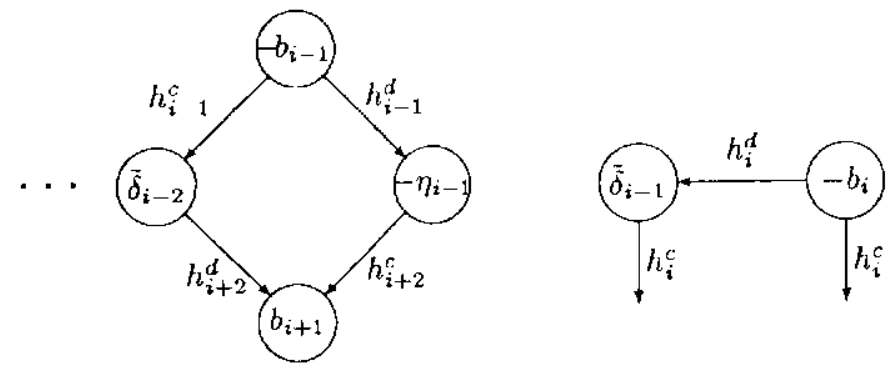

$n=2 i=2 \bmod 4$

$\bar{\delta}_{i-2}=-\delta_{i-2}+\eta_{i-2}$

$\tilde{\delta}_{i-1}=-\delta_{i-1}+\eta_{i}$

$\eta_{i \cdot 1}=\tilde{\delta}_{i-2}-b_{i 1}+b_{i+2}$

Figura 5.7: Hltimos problemas independentes. 
solução, dada em (5.5), é trivialmente inteira. Assim a solução será não negativa e inteira se, e somente se, as seguintes desigualdades são satisfeitas:

$$
\begin{aligned}
b_{0} & \geq 0 \\
b_{1} & \geq b_{0} \\
b_{n} & \geq 0 \\
b_{n-1} & \geq b_{n} .
\end{aligned}
$$

Foi mostrado no Capítulo 3 que o sistema linear correspondendo à rede ciclo da Figura 5.8 tem uma soluçấo inteira não negativa se, e somente se, as seguintes condições se verificam:

$$
\begin{aligned}
-\theta+\alpha+d+\xi & =0 \\
d & \geq-\alpha \\
\theta & >\alpha
\end{aligned}
$$

Além disso, a solução geral (soluçāo particular mais múltiplos de circulações elementares) para este sistema linear é $(x, y, z, w)=(\alpha, \theta-\alpha, d, 0)+m(1,-1,-1,1)$. O número de soluções inteiras não-negativas é o número de valores inteiros admissíveis para $m$, isto é, aqueles para os quais $(x, y, z, w)$ é não negativo. Note que (5.8) está satisfeita por construção para todas as redes produzidas pela divisão, exceto a última rede obtida na decomposição da rede original quando $n=2 i+1$. Assim para $n=2 i+1$, uma condição no conjunto de condições necessárias e suficientes para a existência de soluções inteiras não negativas de (5.1) é

$$
\begin{aligned}
0 & =\tilde{\delta}_{i-1}+(-1)^{i+1} b_{i}+(-1)^{i} b_{i+1}+(-1)^{i} \delta_{i} \\
& =\sum_{k=0}^{i-1}\left((-1)^{k+1} b_{k}+(-1)^{k} b_{n} k\right)+\sum_{k=1}^{i-1}(-1)^{k} \tilde{\delta}_{k}+(-1)^{i+1} b_{i}+(-1)^{i} b_{i+1}+(-1)^{i} \delta_{i} \\
& =\sum_{k=0}^{i}\left((-1)^{k+1} b_{k}+(-1)^{k} b_{n-k}\right)+\sum_{k=1}^{i}(-1)^{k} \delta_{k}
\end{aligned}
$$

Substituindo os valores de $b$ e $\delta$ dados por (5.3) na equação (5.10) obtemos a igualdade de Poincaré-Hopf (2.7), que deve ser satisfeita no caso $n=2 i+1$.

Se $n=2 i=0 \bmod 4$, então foi mostrado no Capítulo 3 que o víltimo sistema linear, que envolve $h_{i}^{c}, h_{i}^{d}$ e $\beta^{i}$ (ver Figura 5.7), tem uma solução inteira não negativa se, e 


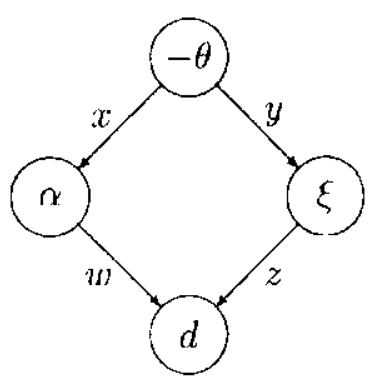

Figura 5.8: Exemplo genérico de ciclo de rede.

somente se, as seguintes desigualdades são satisfeitas:

$$
\begin{aligned}
& b_{i} \geq-\tilde{\delta}_{i-1} \\
& b_{i} \geq \tilde{\delta}_{i-1}
\end{aligned}
$$

A solução geral deste sistema é $\left(h_{i}^{c}, \beta^{i}, h_{i}^{d}\right)=\left(\tilde{\delta}_{i-1}, b_{i}-\bar{\delta}_{i-1}, 0\right)+m(1,-2,1)$. Novamente, o número de soluções inteiras não-negativas é o número de valores inteiros admissíveis para $m$, que, neste caso, são aqueles para os quais $\left(h_{i}^{c}, \beta^{i}, h_{i}^{d}\right)$ é não negativo.

No caso $n=2 i=2 \bmod 4$, o último sistema tem a solução única $\left(h_{i}^{c}, h_{i}^{d}\right)=\left(\left(b_{i}-\right.\right.$ $\left.\left.\tilde{\delta}_{i \cdot 1}\right) / 2,\left(b_{i}+\tilde{\delta}_{i-1}\right) / 2\right)$, que é não negativa e inteira se, e somente se,

$$
\begin{aligned}
b_{i}-\tilde{\delta}_{i-1} & \text { é } \\
b_{i} & \geq-\tilde{\delta}_{i-1} \\
b_{i} & \geq \tilde{\delta}_{i-1} .
\end{aligned}
$$

Note que temos os meios para construir soluções para todos os sistemas, e logo, para o sistema original. Com a exceção dos sistemas com solução única, gerar soluções envolve adicionar múltiplos das circulações elementares.

Finalmente, substituindo(5.3) (ou (5.4) nas condiçöes apropriadas, dentre as obtidas $(5.7),(5.7),(5.9),(5.8),(5.11),(5.12)$ e (5.13), concluímos que o sistema $h_{\kappa}^{\text {cd }}$ tem uma soluçāo se, e somente se, as desigualdades de Poincaré-Hopf (2.20)-(2.16) são satisfeitas.

As Proposiçōes 5.1.1 e 5.1.2 implicam no Teorema 5.0.5 e conseqüentemente parte do Teorema 5.0.6, já que cada explusão de um vértice corresponde a uma solução inteira não negativa do sistema $h_{\kappa}^{c d}$.

Em outras palavras, mostramos que se cada vértice de um grafo de Lyapunov $L\left(h_{0}, \ldots, h_{n}, \kappa\right)$ satisfaz as desigualdades de Poincaré-Hopf para blocos isolantes (2.20) (2.16), o grafo admite uma continuação a um grafo do tipo Morse com cycle rank 
maior ou igual a $\kappa$. Considerando a implosão do grafo $L\left(h_{0}, \ldots, h_{n}, \kappa\right)$ é fácil ver que $\kappa<\min \left\{h_{1}-\left(h_{0}-1\right), h_{n-1}-\left(h_{n}-1\right)\right\}$.

Dado $L\left(h_{n}, \ldots, h_{n}, \kappa\right)$ satisfazendo as desigualdades de Poincaré-Hopf para variedades fechadas (2.21)-(2.23), o conjunto de todas as possíveis continuações determinam a família $\cup_{j \geq \kappa} \mathcal{L}\left(h_{0}, \ldots, h_{n}, j\right)$ de grafos de Lyapunov $L\left(h_{0}, \ldots, h_{n}, j\right), \kappa \leq j \leq \min \left\{h_{1}-\left(h_{0}-1\right)\right.$, $\left.h_{n-1}-\left(h_{n}-1\right)\right\}$.

O número de continuações, isto é, o número de fluxos inteiros não negativos, foi calculado no Capítulo 3 para $\kappa=0$. Pela Proposição 5.1.2, este número é o número de múltiplos admissíveis da circulação elementar adequada em cada subproblema independente. Como os valores de $h_{1}^{c}, h_{1}^{d}, h_{2 i}^{c}$ e $h_{2 i}^{d}$ são unicamente determinados, isto é o número de fluxos inteiros não negativos das redes menores obtidos após a eliminaçāo destas quatro variáveis do sistema de equações (5.1) ou (5.2). é fácil verificar que esta eliminação resultará na mesma subrede, indenpendentemente do valor de $\kappa$. Assim, o número total de continuações é simplesmente o número de valores possíveis de $\kappa\left(1+\min \left\{h_{1}-\left(h_{0}-1\right), h_{n-1}-\left(h_{\mathfrak{n}}-1\right)\right\}\right)$ vezes o número de continuações para $\kappa=0$.

\subsection{Resultados sobre as desigualdades de Morse}

Como foi mostrado na seção anterior, todo grafo abstrato de Lyapunov $L\left(h_{0}, \ldots, h_{n}, \kappa\right)$ que satisfaz as desigualdades de Poincaré-Hopf para variedades fechadas (2.21)-(2.23) podem se continuados a algum grafo na família $\cup_{j>\kappa} \mathcal{L}\left(h_{0}, \ldots, h_{n}, j\right)$ de grafos de Lyapunov $L\left(h_{0}, \ldots, h_{n}, j\right), \kappa \leq j \leq \min \left\{h_{1}-\left(h_{0}-1\right), h_{n-1}-\left(h_{n}-1\right)\right\}$. Cada grafo nesta família pode ser associado a uma solução inteira não negativa para o sistema $h_{\kappa}^{\text {cd }}$ adequado.

Também, usando $B_{j}^{-}=B_{j}^{+}, e^{-}=h_{0}$ e $e^{+}=h_{n}$ obtemos as versões particulares (5.14) e (5.15) dos sistemas (4.1) e (4.2). Logo, o Corolário 5.2.1 segue diretamente da Proposição 5.1.2.

Corolário 5.2.1 Os sistemas abaixo tem uma solução inteira não negativa $\left(h_{1}^{c} h_{1}^{d}, \ldots\right.$, $\left.h_{n}^{c}{ }_{1} h_{n}^{d}{ }_{1}\right)$ se, e somente se, as desigualdades de Poincaré. Hopf para variedades fechadas 
(2.21)-(2.23) são satisfeitas.

$$
\begin{gathered}
n=2 i+1\left\{\begin{array}{l}
h_{0}-1-h_{1}^{c}+\kappa=0 \\
\left\{h_{j}=h_{j}^{c}+h_{j}^{d}, j=1, \ldots, 2 i\right. \\
h_{n}-1-h_{2 i}^{d}+\kappa=0
\end{array}\right. \\
\left\{\begin{array}{l}
h_{1}^{d}-h_{2}^{c}-h_{2 i}^{c}+h_{2 i}^{d} \quad=0 \\
h_{2}^{d}-h_{3}^{c}-h_{2 i-1}^{c}+h_{2 i-2}^{d}=0 \\
\vdots \\
h_{i}^{d}-h_{i+1}^{c}=0
\end{array}\right. \\
n=2 i\left\{\begin{array}{l}
h_{0}-1-h_{1}^{c}+\kappa=0 \\
\left\{\begin{array}{l}
h_{j}=h_{j}^{c}+h_{j}^{d}+\beta^{i}, \quad j=1, \ldots, 2 i-1, \beta^{i}=0 \text { se } j \neq i e 2 i \neq 0 \bmod 4 \\
h_{n}-1-h_{2 i-1}^{d}+\kappa=0
\end{array}\right. \\
\left\{\begin{array}{l}
h_{1}^{d}-h_{2}^{c}-h_{2 i-1}^{c}+h_{2 i-2}^{d}=0 \\
h_{2}^{d}-h_{3}^{c}-h_{2 i-2}^{c}+h_{2 i-3}^{d}=0 \\
\vdots \\
h_{i-1}^{d}-h_{i}^{c}-h_{i+1}^{c}+h_{i}^{d}=0
\end{array}\right.
\end{array}\right.
\end{gathered}
$$

Nos referimos aos sistemas acima como sistemas $h_{\kappa}^{c d}$ reduzidos.

Vale a pena enfatizar que a prova construtiva da equivalência entre a existência de soluçōes inteiras não negativas para o sistema reduzido $h_{\kappa}^{c d}$ e a viabilidade das desigualdades de Poincaré-Hopf (2.21) (2.23) também produz os métodos para construir todas as soluções do sistema reduzido $h_{\kappa}^{c d}$, um fato que será explorado na Seção 5.3 .

A prova que as desigualdades de Poincaré-Hopf implicam as desigualdades de Morse é também construtiva. Produziremos fórmulas para produzir um vetor de números de Betti $\left(\gamma_{0}, \ldots, \gamma_{n}\right)$ que satisfaz as desigualdades de Morse de solıçöes básicas do sistema $h^{c d}$ reduzido (apropriado).

Primeiro consideramos o caso $n=2 i+1$. Seja $h^{c d}$ uma soluçāo inteira não negativa do sistema $h_{\kappa}^{\text {cd }}$ reduzido (5.14). Assumiremos que esta solução satisfaz a condição de complementaridade

$$
h_{j}^{c} h_{2 i+1-j}^{d}=0, \quad \text { para } j=2, \ldots, i
$$


Isto segue do fato que se $(\hat{x}, \hat{y}, \hat{z}, \hat{w})$ é uma solıção inteira não negativa do problema do fluxo na rede ciclo descrita na Figura 5.8, então $\left(x^{\prime}, y^{\prime}, z^{\prime}, w^{\prime}\right)=(\hat{x}, \hat{y}, \hat{z}, \hat{w})-$ $\min \{x, w\}(-1,1,1,-1)$ é ainda uma outra solução inteira não negativa, satisfazendo $x^{\prime} w^{\prime}=0$. Assim, dada uma solução inteira não negativa arbitrária $h^{c d}$ podemos transformá-la em uma soluçāo inteira não negativa satisfazendo (5.16) somando múltiplos adequados das circulaçōes elementares. Observe que esta nova solução é uma solução básica.

Suponhamos $n=2 i=0$ mod 4 . Sem perda de generalidade podemos considerar uma solução inteira não negativa do sistema $h_{\kappa}^{c d}$ reduzido (5.15) que satisfaz (5.16). O argumento para $2 \leq j \leq i-1$ é o mesmo que antes. Agora se $\left(\hat{h}_{i}^{c}, \hat{\beta}^{i}, \hat{h}_{i}^{d}\right)$ é uma solução inteira não negativa do último problema independente descrito na Figura 5.7, então $\left(\tilde{h}_{i}^{c}, \tilde{\beta}^{i}, \tilde{h}_{i}^{d}\right)=\left(\hat{h}_{i}^{c}, \hat{\beta}^{i}, \hat{h}_{i}^{d}\right)+\min \left\{\hat{h}_{i}^{c}, \hat{h}_{i}^{d}\right\}(-1,2,-1)$ é uma outra solução deste subproblema que satisfaz (5.16).

Para cuidar do caso $n=2 i=2 \bmod 4$, introduzirnos o sistema auxiliar abaixo.

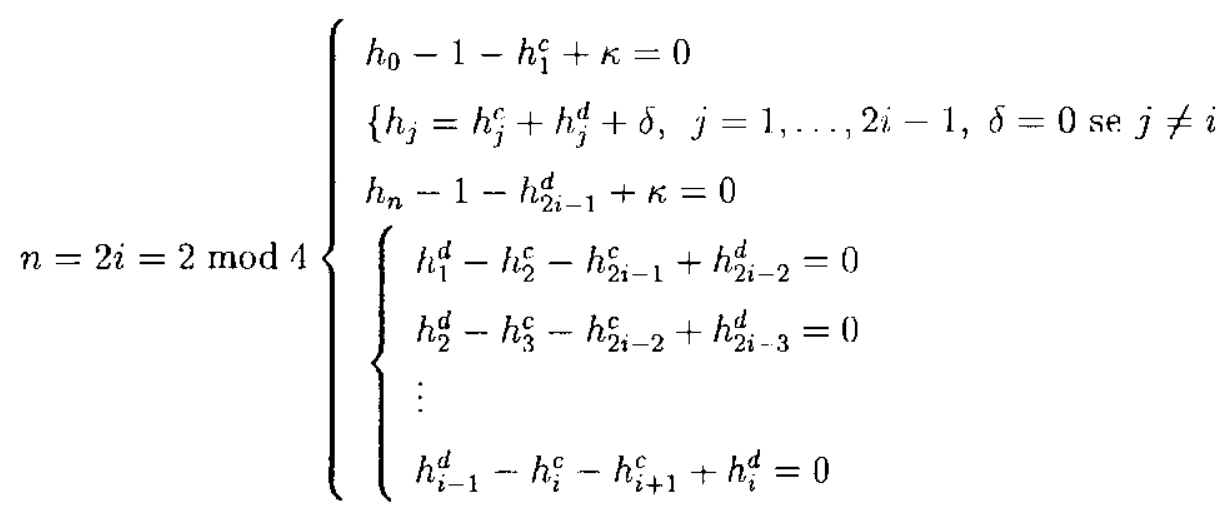

Se (5.15) tem uma solução inteira não negativa $\left(h_{1}^{c}, h_{1}^{d}, \ldots, h_{i}^{c}, h_{i}^{d}, \ldots, h_{2 i}^{c}, h_{2 i}^{d}\right)$, então (5.17) tem a solução $\left(h_{1}^{c}, h_{1}^{d}, \ldots, h_{i}^{c}, 0, h_{i}^{d}, \ldots, h_{2 i}^{c}, h_{2 i}^{d}\right)$ (simplesmente faça $\delta=0$ ). A vantagem de introduzir (5.17) é que agora temos espaço de manobra para transformar uma dada solução inteira não negativa $\left(h_{1}^{c}, h_{1}^{d}, \ldots, h_{i}^{c}, 0, h_{i}^{d}, \ldots, h_{2 i}^{c}, h_{2 i}^{d}\right)$ de (5.17) em uma que satisfaça (5.16) da mesma forma como feito no caso $n=2 i=0 \bmod 4$.

Dadas estas soluçōes básicas pode ser mostrado que as fórmulas abaixo definem vetores 
de números de Betti $\left(\gamma_{0}, \ldots, \gamma_{n}\right)$ que satisfazem as desigualdades de Morse.

$$
\begin{gathered}
\gamma_{0}=\gamma_{2 i+1}=1 \\
\gamma_{j}= \begin{cases}h_{1}^{d}-h_{2}^{c}+\kappa, & \text { se } j=1 \\
h_{j}^{d}-h_{j+1}^{c}, & \text { se } 2 \leq j<i \\
h_{i}^{d}, & \text { se } j=i \\
h_{i+1}^{c}, & \text { se } j=i+1 \\
-h_{j-1}^{d}+h_{j}^{c}, & \text { se } i+2 \leq j \leq 2 i-1 \\
-h_{2 i-1}^{d}+h_{2 i}^{c}+\kappa, & \text { se } j=2 i\end{cases} \\
\quad \gamma_{0}=\gamma_{2 i}=1
\end{gathered}
$$

O único papel da condição de complementaridade (5.16) é garantir a nāo negatividade de $\gamma$ definido acima. Outras soluçōes dos sistemas $h_{\kappa}^{c d}$ reduzidos levam a vetores de números de Betti que satisfazem as desigualdades de Morse e as condições de dualidade mas podem ter componentes negativas. Estes vetores de números de Betti não serão considerados neste contexto.

Dado o Corolário 5.2.1, a parte da suficiência do Teorema 5.0.7 é estabelecida pela seguinte proposiçāo, que foi provada no Capítulo 4 para $\kappa=0$.

Proposiçāo 5.2.2 Se o sistema $h_{\kappa}^{c d}$ reduzido tem uma solução inteira não negativa, então existe um vetor de números de Betti inteiro não negativo $\left(\gamma_{0}, \ldots, \gamma_{n}\right)$ que satisfaz as desigualdades de Morse (1) e a desigualdade $\gamma_{1}>\kappa$.

\section{Demonstração:}

Caso $n=2 i+1$.

Por definiçāo (5.18), $\gamma$ é claramente inteiro. As equações $h_{j}^{d}-h_{j+1}^{c}-h_{2 i+1, j}^{c}+h_{2 i-j}^{d}=0$ em (5.14), para $j=1, \ldots, i-1$, implicam $\gamma_{j}=\gamma_{2 i+1-j}$, para $j=1 \ldots, i-1, i+2, \ldots, 2 i$. 


\section{5. Grafos de Lyapunov, Desigualdades de Poincaré-Hopf e Desigualdades de Morse}

Da mesma forma, as equações $h_{i}^{d}-h_{i+1}^{c}=0 \mathrm{em}$ (5.14) implicam que $\gamma_{i}=\gamma_{i+1}$. Portanto o vetor $\gamma$ dado por (5.18) satisfaz as condições $\gamma_{j}=\gamma_{2 i+1-j}$, para $j=0, \ldots, 2 i+1$.

Para mostrar que $\gamma \geq 0$ é suficiente mostrar que $\gamma_{j}$ ou $\gamma_{2 i+1-j}$, para $1 \leq j \leq i-1$, é não negativo, já que $\gamma_{j}=\gamma_{2 i+1-j}$, para $j=0, \ldots, 2 i+1, \gamma_{i}=\gamma_{i+1}=h_{i}^{d} \geq 0$ e $\gamma_{0}=\gamma_{2 i+1}=1$.

A definição de $\gamma$ e (5.16) implicam isto, já que $h_{j+1}^{c} h_{2 i-j}^{d}=0 \Rightarrow\left\{\begin{array}{l}h_{j+1}^{c}=0 \Rightarrow \gamma_{2 i+1-j}=\gamma_{j}=h_{j}^{d}-h_{j+1}^{c}=h_{j}^{d} \geq 0 \\ 011 \\ h_{2 i-j}^{d}=0 \Rightarrow \gamma_{j}=\gamma_{2 i+1-j}=-h_{2 i-j}^{d}+h_{2 i+1-j}^{c}=h_{2 i+1-j}^{c} \geq 0\end{array} \quad\right.$ para $2 \leq j \leq i-1$ e $h_{2}^{c} h_{2 i-1}^{d}=0 \Rightarrow\left\{\begin{array}{l}h_{2}^{r}=0 \Rightarrow \gamma_{2 i}=\gamma_{1}=h_{1}^{d}-h_{2}^{c}-\kappa=h_{1}^{d}+\kappa \geq \kappa \geq 0 \\ \text { ou } \\ h_{2 i-1}^{d}=0 \Rightarrow \gamma_{1}=\gamma_{2 i}=h_{2 i-1}^{d}+h_{2 i}^{c}+\kappa=h_{2 i}^{c}+\kappa \geq \kappa \geq 0\end{array} \quad\right.$ para $2 \leq j<i-1$.

Dado que $h_{j}=h_{j}^{c}+h_{j}^{d}, j=1, \ldots, 2 i$, temos que:

$$
\begin{aligned}
\sum_{j=0}^{2 i+1}(-1)^{j+1} h_{j}= & -h_{0}+h_{1}^{c}+\sum_{j=1}^{2 i-1}(-1)^{j+1}\left(h_{j}^{d}-h_{j+1}^{c}\right)-h_{2 i}^{d}+h_{2 i+1} \\
= & -1+\kappa+\sum_{j=1}^{2 i-1}(-1)^{j+1}\left(h_{j}^{d}-h_{j+1}^{c}\right)-\kappa+1 \\
= & -\gamma_{0}+h_{1}^{d}-h_{2}^{c}+\kappa+\sum_{j=2}^{i-1}(-1)^{j+1}\left(h_{j}^{d}-h_{j+1}^{c}\right) \\
& +(-1)^{i+1} h_{i}^{d}-(-1)^{i+1} h_{i+1}^{c} \\
& +\sum_{j-i+1}^{2 i-2}(-1)^{j+1}\left(h_{j}^{d}-h_{j ! 1}^{c}\right)+h_{2 i-1}^{d}-h_{2 i}^{c}-\kappa+\gamma_{2 i+1} \\
= & -\gamma_{0}+\gamma_{1}+\sum_{j=2}^{i}(-1)^{j+1} \gamma_{j}+(\cdots 1)^{i+1} \gamma_{i}+(--1)^{i+2} \gamma_{i+1} \\
& +\sum_{j-i+1}^{2 i-2}(-1)^{j+1}\left(-\gamma_{j ! 1}\right)+(-1)^{2 i+1} \gamma_{2 i}+\gamma_{2 i+1} \\
= & \sum_{j=0}^{2 i+1}(-1)^{j+1} \gamma_{j} .
\end{aligned}
$$

Logo, provamos a igualdade de Morse, e primeira equação em (1). 
Analogamente, pode ser mostrado que

$$
\begin{aligned}
\sum_{j=0}^{\ell}(-1)^{j+1} h_{j} & =-h_{0}+h_{1}^{c}+\sum_{j=1}^{\ell-1}(-1)^{j+1}\left(h_{j}^{d}-h_{j+1}^{c}\right)+(-1)^{\ell+1} h_{\ell}^{d} \\
& =-h_{0}+h_{1}^{c}+\sum_{j=1}^{\ell}(-1)^{j+1}\left(h_{j}^{d}-h_{j+1}^{c}\right)+(-1)^{\ell+1} h_{\ell+1}^{c} .
\end{aligned}
$$

As equações acima implicam que

$$
\sum_{j=0}^{\ell}(-1)^{j+1} h_{j}= \begin{cases}\sum_{j=0}^{\ell}(-1)^{j+1} \gamma_{j}+(-1)^{\ell+1} h_{\ell}^{d}, & \text { se } i+1 \leq \ell \leq 2 i \\ \sum_{j=0}^{i}(-1)^{j+1} \gamma_{j}, & \text { se } \ell=i \\ \sum_{j=0}^{\ell}(-1)^{j+1} \gamma_{j}+(-1)^{\ell+1} h_{\ell+1}^{c}, & \text { se } 1 \leq \ell \leq i-1 .\end{cases}
$$

Assim, levando em consideração a não negatividade de $\left(h_{1}^{c}, h_{1}^{d}, \ldots, h_{2 i}^{\mathrm{c}}, h_{2 i}^{d}\right)$, obtemos todas exceto a última desigualdade de Morse a partir de (5.20).

Pelo sistema $h_{(\kappa)}^{c d}$ reduzido temos que $h_{0}=h_{1}^{c}-(\kappa-1)$. Como $\gamma_{0}=1$, isto implica que $h_{0}=h_{1}^{c}-\kappa+\gamma_{0}$. Como $h_{1}^{c} \geq \kappa$ se $h_{0} \geq \gamma_{0}$, fica assim estabelecida a última desigualdade de Morse.

\section{Caso $n=2 i$.}

Este caso é análogo ao caso de dimensão ímpar. A integralidade e não negatividade

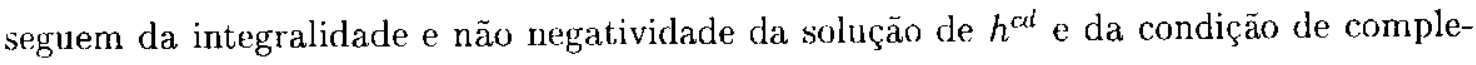
mentaridade. Com relação à satisfação das desigualdades de Morse, daremos detalhes para o caso $2 i=0 \bmod 4$. Os argumentos podem ser facilmente adaptados para o caso $2 i=2 \bmod 4$ simplesmente substituindo $\beta^{2}$ por $\delta$. 
Somando as primeiras $2 i+1$ equações de (5.15) temos

$$
\begin{aligned}
\sum_{j-0}^{2 i}(-1)^{j+1} h_{j}= & -h_{0}+h_{1}^{c}+\sum_{j-1}^{2 i-2}(-1)^{j+1}\left(h_{j}^{d}-h_{j+1}^{c}\right)+(-1)^{i+1} \beta^{i}+h_{2 i-1}^{d}-h_{2 i} \\
= & -1+\kappa+\sum_{j=1}^{2 i-2}(-1)^{j+1}\left(h_{j}^{d}-h_{j+1}^{c}\right)+(-1)^{i+1} \beta^{i}+\kappa-1 \\
= & -\gamma_{0}+h_{1}^{d}-h_{2}^{c}+\kappa+\sum_{j=2}^{i-1}(-1)^{j+1}\left(h_{j}^{d}-h_{j+1}^{c}\right)+(-1)^{i+1} \beta^{i} \\
& +\sum_{j=i}^{2 i-3}(-1)^{j+1}\left(h_{j}^{d}-h_{j+1}^{c}\right)-h_{2 i-2}^{d}+h_{2 i-1}^{c}+\kappa-\gamma_{2 i} \\
= & -\gamma_{0}+\gamma_{1}+\sum_{j=2}^{i \cdots 1}(-1)^{j+1} \gamma_{j}+(-1)^{i+1} \gamma_{i} \\
& +\sum_{j=i}^{2 i-2}(-1)^{j+1}\left(-\gamma_{j+1}\right)+\gamma_{2 i-1}-\gamma_{2 i} \\
= & \sum_{j=0}^{2 i}(-1)^{j+1} \gamma_{j} .
\end{aligned}
$$

Logo, provamos a igualdade de Morse.

As próximas equações são obtidas de modo análogo:

$$
\begin{aligned}
& \sum_{j=0}^{\ell}(-1)^{j+1} h_{j}=\left\{\begin{array}{c}
-h_{0}+h_{1}^{c}+\sum_{j-1}^{\ell-1}(-1)^{j+1}\left(h_{j}^{d}-h_{j+1}^{c}\right)+(-1)^{i+1} \beta^{i}+(-1)^{\ell+1} h_{\ell}^{d}, \\
\text { se } i \leq \ell \leq 2 i-1 \\
-h_{0}+h_{1}^{c}+\sum_{j-1}^{\ell}(-1)^{j+1}\left(h_{j}^{d}-h_{j+1}^{c}\right)+(-1)^{\ell+1} h_{\ell+1}^{c}, \\
\text { se } 1 \leq \ell<i
\end{array}\right. \\
& = \begin{cases}\sum_{j-0}^{\ell}(-1)^{j+1} \gamma_{j}+(-1)^{\ell+1} h_{\ell}^{d}, & \text { se } i \leq \ell \leq 2 i-1 \\
\sum_{j=0}^{\ell}(-1)^{j+1} \gamma_{j}+(-1)^{\ell+1} h_{\ell+1}^{c}, & \text { se } 1 \leq \ell \leq i-1 .\end{cases}
\end{aligned}
$$

A não negatividade de $\left(h_{1}^{c}, h_{1}^{d}, \ldots, h_{2 i}^{c}, h_{2 i}^{d}\right)$ implica em todas exceto a última desigualdade de Morse. A última desigualdade é estabelecida exatamente como no caso $n=2 i+1$.

Provamos a seguir a necessidade das desigualdades de Poincaré-Hopf no Teorema 5.0.7 para as desigualdades de Morse serem satisfeitas. 


\section{Conclusão da prova do Teorema 5.0.7:}

Pela desigualdade (1) de (1) temos que

$$
h_{1} \geq \gamma_{1}-\gamma_{0}+h_{0}
$$

Usando o fato que $\gamma_{1} \geq \kappa$ temos

$$
h_{1} \geq \kappa-1+h_{0}
$$

Pela dualidade dos índices de Conley e usando o fato que $\gamma_{n-1} \geq \kappa$ temos

$$
h_{n-1} \geq \kappa-1+h_{n}
$$

e portanto obtemos as desigualdades (1) de (2.21).

Para $j=2, \ldots, i$, segue o procedimento descrito abaixo. Da desigualdade (j) de (1) temos que

$$
\begin{aligned}
h_{j} & \geq \gamma_{j}-\gamma_{j-1}+\ldots \ldots \pm \gamma_{2} \pm \gamma_{1} \pm \gamma_{0}+h_{j-1}-h_{j-2}+\ldots \ldots \pm h_{2} \pm h_{1} \pm h_{0} \\
& \geq-\gamma_{j-1}+-\ldots \pm \gamma_{2} \pm \gamma_{1} \pm \gamma_{0}+h_{j-1}-h_{j-2}+-\ldots \pm h_{2} \pm h_{1}+h_{0} .
\end{aligned}
$$

Logo,

$$
h_{j}+\gamma_{j \cdot 1}-\gamma_{j-2}-+\ldots \pm \gamma_{2} \pm \gamma_{1} \pm \gamma_{0} \geq h_{j-1}-h_{j \cdots 2}+-\ldots \pm h_{2} \pm h_{1} \pm h_{0}
$$

Também segue das desigualdades de Morse duais que

$$
\gamma_{j-1} \cdot \gamma_{j-2}+-\ldots \pm \gamma_{2} \pm \gamma_{1} \pm \gamma_{0} \leq h_{n}(j \cdot 1)-h_{n-(j-2)}+-\ldots \pm h_{n-2} \pm h_{n \cdots 1} \pm h_{n}
$$

Substituindo (5.23) em (5.22) temos que

$$
\begin{gathered}
h_{j}+h_{n \cdots(j-1)}-h_{n-(j-2)}+-\ldots \pm h_{n-2} \pm h_{n-1} \pm h_{n} \geq h_{j-1}-h_{j-2}+\cdots \ldots \pm h_{2} \pm h_{1} \pm h_{0} \\
\Rightarrow h_{j} \geq-\left(h_{n-(j-1)} \cdots h_{j-1}\right)+\left(h_{n-(j-2)}-h_{j-2}\right)-+\ldots \pm\left(h_{n-1}-h_{1}\right) \pm\left(h_{n}-h_{0}\right)
\end{gathered}
$$

Multiplicando por (-1) temos que

$$
-h_{j} \leq\left(h_{n-(j-1)}-h_{j-1}\right)-\left(h_{n-(j-2)}-h_{j-2}\right)+-\ldots+\left(h_{n-1}-h_{1}\right)+\left(h_{n}-h_{0}\right)
$$

Usando a dualidade dos índices de Conley temos que

$$
-\bar{h}_{j} \leq\left(\bar{h}_{n-(j-1)}-\bar{h}_{j-1}\right)-\left(\bar{h}_{n \cdots(j-2)}-\bar{h}_{j-2}\right)+-\ldots \pm\left(\bar{h}_{n-1}-h_{1}\right) \pm\left(\bar{h}_{n}-\bar{h}_{0}\right)
$$


Finalmente multiplicando por $(-1)$ e usando a dualidade dos índices de Conley, $\bar{h}_{j}=$ $h_{n-j}$, temos que

$$
h_{n-j} \geq\left(h_{n-(j-1)}-h_{j} 1\right)-\left(h_{n-(j-2)}-h_{j-2}\right)+-\ldots \pm\left(h_{n-1}-h_{1}\right)+\left(h_{n}-h_{0}\right)
$$

Logo obtemos as desigualdades (j) de $(2.21) \operatorname{com} \mathrm{j}=2, \ldots, \mathrm{i}-1$.

Para obter as desigualdades nas dimensões médias o procedimento é análogo. Contudo, devemos observar que se $n=2 i$ temos que $n-i=i$ e $\log h_{i}$ é dual a ele mesmo. Se $n=2 i+1$ o dual de $h_{i}$ é $h_{i+1}$.

Apresentaremos um exemplo na Figura 5.9 de um grafo de Lyapunov em dimensão 7, que pode possivelmente representar um fluxo sobre ıma variedade $M$ de dimensão 7 . Se este é o caso, lembre que cada aresta do grafo representa uma variedade $N$ conexa e fechada de dimensāo 6 vezes um intervalo e logo é necessário somente rotular com os números de Betti $\left(\beta_{1}, \beta_{2}, \beta_{3}\right)$ da variedade $N$. Na Figura 5.9 o grafo abstrato de Lyapunov $L\left(h_{0}, h_{1}, \ldots, h_{7}, \kappa\right)=L(2,4,1,2,1,1,4,1, \kappa=2)$ é apresentado. Teoricamente, cada vértice $v$ deve ser rotulado com $\left(h_{0}(v), h_{1}(v), \ldots, h_{7}(v), \kappa_{v}\right)$. Contudo, se este vetor contém muitos zeros, por exemplo, $(0,1,0,0,0,0,0,0, \kappa=0)$ adotamos a notação alternativa $h_{1}=1$ conforme mencionamos na introdução. Sempre que um vértice é rotulado com $h_{j}=1, j \neq 0,7$, também especificaremos se o vértice é $j$-desconectante, $j$-d, on $(j-1)$-conectante, $(j-1)$-c.

$$
\begin{aligned}
& \begin{aligned}
h_{7} & =1 \\
& v-\mathrm{c} \quad h_{1}=1
\end{aligned}\left\{\begin{array}{l}
(0,0,0) \\
\left(0,2,1,2,1,1,3,0, \kappa_{v}=1\right) \\
(0,0,0)
\end{array}\right. \\
& (0,0,0), \quad(0,0,0) \\
& \text { 6-d } h_{6}=1 \sum_{(0,0,0)}^{\left(0, c, h_{1}=1\right.}
\end{aligned}
$$

Figura 5.9: Grafo abstrato de Lyapunov $L(2,4,1,2,1,1,4,1, \kappa=2)$

Na Figura 5.10 apresentamos duas possíveis continuaçōes de $L(2,4,1,2,1,1,4,1, \kappa=$ 
2) que pertencem à família $\mathcal{L}(2,4,1,2,1,1,4,1, \kappa=2)$. Note que qualquer grafo em $\mathcal{L}(2,4,1,2,1,1,4,1, \kappa=2)$ deve ter cycle number $2 \leq \leq \min \left\{h_{1}-\left(h_{0}-1\right), h_{6}-\left(h_{7}-1\right)\right\}=$ 3. Lembre que $\kappa$ está relacionado ao primeiro número de Betti $\gamma_{1}$ da variedade $M$ pela desigualdade $\gamma_{1} \geq \kappa$.
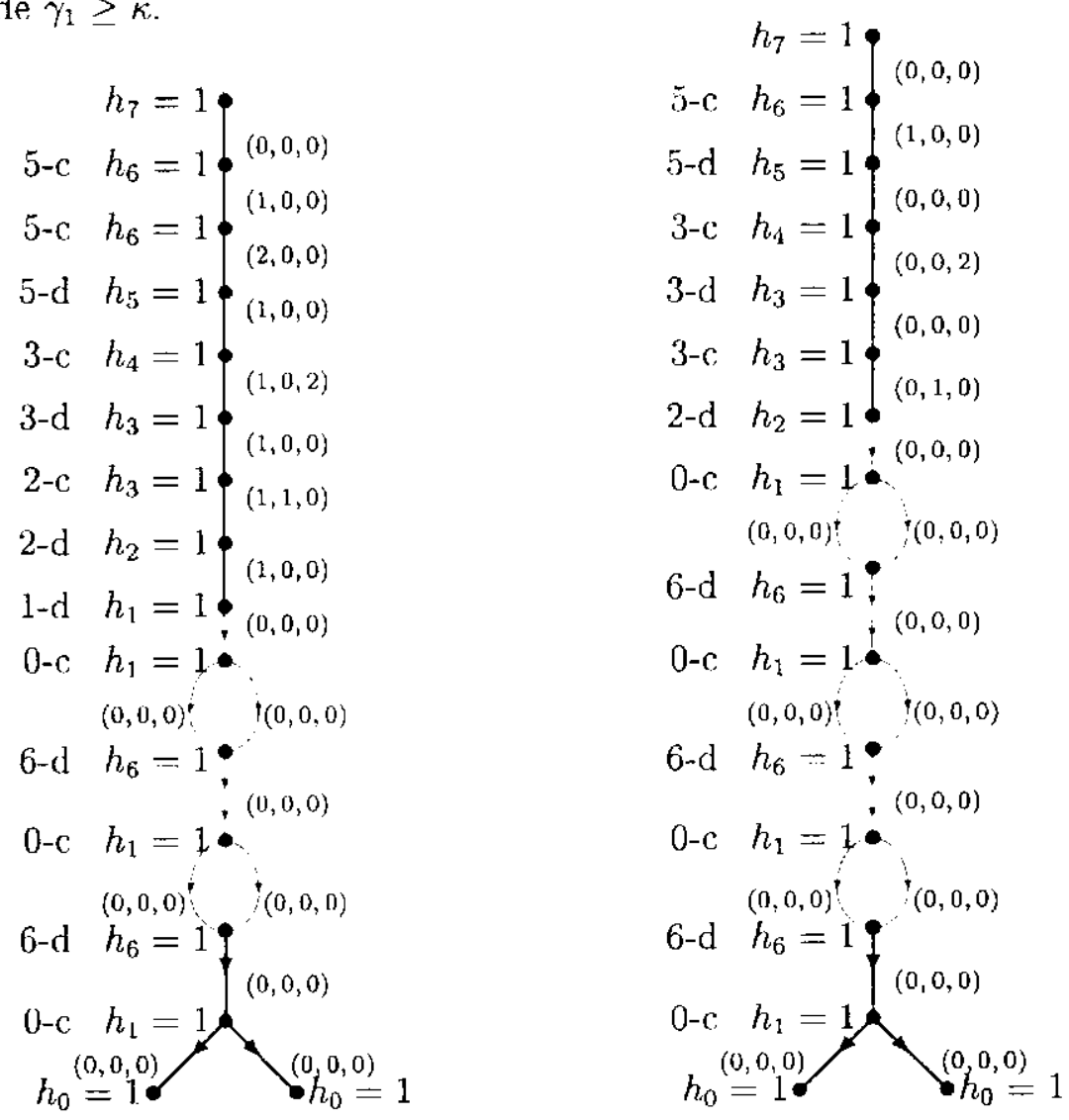

Figura 5.10: Grafos na família $\mathcal{L}(2,4,1,2,1,1,4,1, \kappa=2)$.

Finalmente, na Figura 5.11 apresentamos a implosão de $L(2,4,1,2,1,1,4,1, \kappa=2)$.

\subsection{Politopo de Morse}

Considere um conjunto de dados de índice pré-fixado $\left(h_{0}, h_{1}, \ldots, h_{n}\right)$ e scja $\kappa$ um inteiro no intervalo $\left[0, \min \left\{h_{1}-\left(h_{0}-1\right), h_{\eta-1}-\left(h_{n}-1\right)\right\}\right]$. A permissão da existência de ciclos adiciona uma desigualdade extra $\gamma_{1} \geq \kappa$ ao conjunto das desigualdades de Morse. Este conjunto maior de desigualdades, mais as restriçōes de contorno $\gamma_{0}=\gamma_{n}=1$, as restrições 


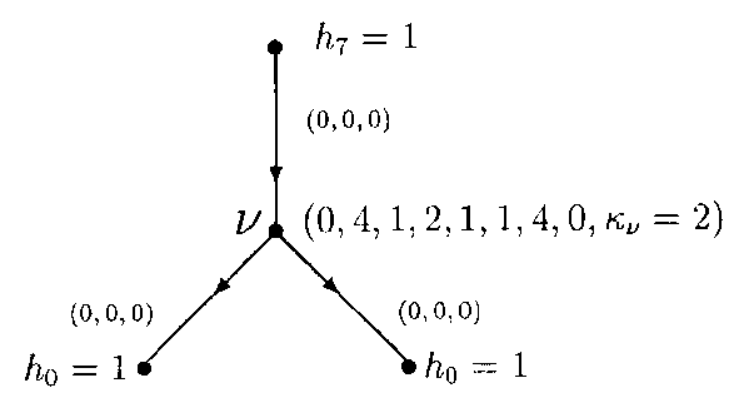

Figura 5.11: Implosão de $L(2,4,1,2,1,1,4,1, \kappa=2)$.

de dualidade $\gamma_{j}=\gamma_{n-j}$, para $j=0, \ldots, n$, e as restriçōes não negativas $\gamma \geq 0$, definem um poliedro $\mathcal{P}_{\kappa}\left(h_{0}, \ldots, h_{n}\right)$, que por simplicidade nos referimos como $\mathcal{P}_{\kappa}$, em $\mathbb{R}^{n+1}$, que scrá o assunto desta seção. Este poliedro é de fato um politopo, isto é, um poliedro limitado, já que $0 \leq \gamma \leq h_{j}$ (temos o limite superior pelas desigualdades $(j)$ e $(j-1)$ de $(1)$ ), para $1 \leq j \leq n-1$. Muito será herdado do estudo do politopo de Morse feito no capítulo anterior, já que a adição da desigualdade $\gamma>\kappa$ conduzirá a pequenas modificações do politopo.

O conjunto de soluções não negativas do sistema $h_{\kappa}^{c d}$ reduzido (casos especiais de (4.1) e (4.2)) também constituem um politopo. É notável que ambos os politopos tenham vértices inteiros, e que exista uma relação (apesar não 1-a-1) entre os elementos inteiros em cada politopo.

\subsubsection{Caso $n$ ímpar}

O estudo de $\mathcal{P}_{\kappa}$ pode ser significantemente simplificado considerando o politopo $\mathcal{P}_{\kappa}^{r}$ reduzido, obtido eliminando $\gamma_{0}, \gamma_{i+1}, \ldots, \gamma_{2 i+1}$, usando as equaçöes de contorno e dualidade. Estas condições implicam que

$$
\sum_{j=0}^{2 i+1-k}(-1)^{j+1} \gamma_{j}=-1+\sum_{j=1}^{\min \{k-1,2 i+1-k\}}(-1)^{j+1} \gamma_{j}
$$


Portanto, $\mathcal{P}_{\kappa}^{r}$ é o conjunto de $\gamma^{r} \in \mathbb{R}^{i}$ que satisfaz

$$
\begin{aligned}
0=\sum_{j=0}^{2 i+1}(-1)^{j+1} h_{j}, \quad 0 \leq h_{0}-1, \quad 0 \leq h_{2 i+1}-1 \\
\sum_{j-1}^{\min \{k-1,2 i+1-k\}}(-1)^{j+1} \gamma_{j} \leq 1+\sum_{j=0}^{2 i+1-k}(-1)^{j+1} h_{j}, \text { para } 2 \leq k \leq 2 i, k \text { par } \\
\sum_{j-1}^{\min \{k-1,2 i+1-k\}}(-1)^{j+1} \gamma_{j} \geq 1+\sum_{j=0}^{2 i+1-k}(-1)^{j+1} h_{j}, \text { para } 2 \leq k \leq 2 i, k \text { impar } \\
\gamma^{r} \geq 0 \\
\gamma_{1} \geq \kappa .
\end{aligned}
$$

Claramente, existe uma correspondência 1-a-1 entre $\gamma^{r}=\left(\gamma_{1}, \gamma_{2}, \ldots, \gamma_{i}\right)$ em $\mathcal{P}_{\kappa}^{r}$ e $\gamma=$ $\left(\gamma_{0}, \gamma_{1}, \ldots, \gamma_{2 i+1}\right)$ em $\mathcal{P}_{\kappa}$

A proposição seguinte estende os resultados obtidos no Capítulo 4 para $\kappa=0$.

Proposição 5.3.1 O politopo $\mathcal{P}_{\kappa}^{r}$ dado por (5.27) satisfaz as seguintes propriedades:

1. Os vértices de $\mathcal{P}_{\kappa}^{r}$ são inteiros.

2. Cada vértice de $\mathcal{P}_{\kappa}^{r}$ pertence a uma das faces: $\mathcal{F}_{t}=\left\{\gamma \in \mathcal{P}_{\kappa}^{r} \mid \sum_{j=1}^{i}(-1)^{j+1} \gamma_{j}=\right.$ $\left.1+\sum_{j=1}^{i}(-1)^{j+1} h_{j}\right\}$ ou $\mathcal{F}_{0}=\left\{\gamma \in \mathcal{P}_{\kappa}^{r} \mid \gamma_{i}=0\right\}$

3. Se $\tilde{\gamma}^{r} \in \mathcal{F}_{t}$, então $\left(\tilde{\gamma}_{1}, \ldots, \tilde{\gamma}_{i-1}, 0\right) \in \mathcal{F}_{0}$, isto é, $\mathcal{F}_{0}$ é a projeçäo de $\mathcal{F}_{t}$ sobre o plano $\gamma_{i}=0$.

4. Cada $\gamma^{r}$ (inteiro) em $\mathcal{F}_{t}$ corresponde a um $h^{\text {cd }}$ (inteiro) näo negativo satisfazendo (5.14).

\section{Demonstração:}

1. No Capitulo 4 foi mostrado que a matriz de coeficientes correspondendo a todas, exceto a última, as desigualdades em (5.27) é totalmente unimodular. Mas a linha de coeficientes correspondendo à última desigualdade é $(1,0, \ldots, 0)$. Anexando uma tal linha a uma matriz totalmente unimodular preserva esta propriedade. Como o lado direito é obviamente inteiro, os vértices de $\mathcal{P}_{\kappa}^{r}$ são inteiros. 
2. A prova para a proposição correspondente no Capítulo 4 pode ser aplicada literalmente aqui.

3. Apenas duas das desigualdades em (5.27) contém $\gamma_{i}: \gamma_{i} \geq 0$ e. $(-1)^{i+1} \sum_{j=1}^{i}(-1)^{j+1} \gamma_{j} \leq(-1)^{i+1}\left(1+\sum_{j-0}^{i}(-1)^{j+1} h_{j}\right)$ (a desigualdade obtida quando $k=i+1)$. A última é equivalente a

$$
(-1)^{i+1}(-1)^{i+1} \gamma_{i}=\gamma_{i} \leq(-1)^{i+1}\left(1-h_{0}+h_{1} \sum_{j=2}^{i}(-1)^{j+1}\left(h_{j}-\gamma_{j}\right)\right)
$$

Para $\tilde{\gamma}^{r} \in \mathcal{F}_{t}$ a desigualdade acima

$$
\tilde{\gamma}_{i}=\tilde{u}=(-1)^{i+1}\left(1-h_{0}+h_{1} \sum_{j=2}^{i}(-1)^{j+1}\left(h_{j}-\tilde{\gamma}_{j}\right)\right) .
$$

Portanto $\left(\tilde{\gamma}_{1}, \ldots, \tilde{\gamma}_{i-1}, \xi\right) \in \mathcal{P}_{\kappa}^{r}$ para $0 \leq \xi \leq \tilde{u}$.

4. Dado um $\gamma^{r}$ (inteiro) $\mathrm{cm} \mathcal{F}_{t}$, considere sua extensāo natural $\gamma$ in $\mathcal{P}_{\kappa}$, isto é, seja $\gamma_{0}=\gamma_{2 i+1}=1$ e $\gamma_{2 i+1-\ell}=\gamma_{\ell}$, para $1 \leq \ell \leq i$. A prova da Proposiçāo 4.1.3 do Capítulo 4 pode ser facilmente adaptada para mostrar que o vetor $h^{\text {cd }}$ definido abaixo é uma solução (inteira) não negativa para (5.14):

$$
\begin{aligned}
& h_{2 i}^{d}=-\sum_{j=0}^{2 i}(-1)^{j+1}\left(h_{j}-\gamma_{j}\right)+\kappa, \\
& h_{2 i+1-\ell}^{d}=(-1)^{\ell} \sum_{j-0}^{2 i+1 \cdot \ell}(-1)^{j+1}\left(h_{j}-\gamma_{j}\right), \text { para } 2 \leq \ell \leq i \\
& h_{2 i+2 \ell}^{c}=(-1)^{\ell} \sum_{j=0}^{2 i+1} \ell(-1)^{j+1}\left(h_{j}-\gamma_{j}\right), \text { para } i+2 \leq \ell \leq 2 i \\
& h_{1}^{c}=h_{0}-\gamma_{0}+\kappa, \\
& h_{1}^{d}=\gamma_{1}+h_{2}^{c}-\kappa \\
& h_{\ell}^{d}=\gamma_{\ell}+h_{\ell+1}^{c}, \quad \text { para } 2 \leq \ell \leq i-1 \\
& h_{\ell}^{c}=\gamma_{\ell}-h_{\ell-1}^{d}, \quad \text { para } i+2 \leq \ell \leq 2 i-1 \\
& h_{2 i}^{c}=\gamma_{2 i}+h_{2 i-1}^{d}-\kappa \text {, } \\
& h_{i}^{d}=\gamma_{i} \\
& h_{i+1}^{r}=\gamma_{i+1} \text {. }
\end{aligned}
$$


A Proposição 5.3.1 assim implica que $\mathcal{P}_{\kappa}$ é o casco convexo dos vetores dos números de Betti inteiros não negativos que satisfazem as desigualdades de Morse e a desigualdade $\gamma_{1} \geq \kappa$. Suponha que exista soluçōes inteiras não negativas de (5.14). Seja $\vec{h}^{c d}$ a solução inteira não negativa do sistema reduzido $h_{0}^{c d}$ que satisfaz as condiçōes de complementaridade (5.16). Seja $\bar{\gamma}$ o vetor dos números de Betti em $\mathcal{P}_{\kappa}$ construídos de $\bar{h}^{\text {cd }}$ usando $(5.18)$.

Proposiçāo 5.3.2 Suponha que o sistema reduzido $h_{\kappa}^{c d}(5.14)$ admite soluções não negativas. $\mathcal{P}_{\kappa}^{r}$ pode ser reescrito como

$\mathcal{P}_{\kappa}^{r}=\left\{0 \leq \gamma^{\tau} \in \mathbb{R}^{i} \mid \gamma_{1} \geq \kappa, e(-1)^{k+1} \sum_{j=0}^{k}(-1)^{j+1} \gamma_{j} \leq(-1)^{k+1} \sum_{j=0}^{k}(-1)^{j+1} \bar{\gamma}_{j}\right.$, para $\left.1 \leq k \leq i\right\}$.

Além disso, $\bar{\gamma}$ é o vetor máximo de $\mathcal{P}_{\kappa}^{r}$, componente a componente.

Demonstraçāo: Esta proposição foi provada no Capítulo 4 para $\kappa=0$. Assim a primeira afirmação é trivialmente verdadeira, já que $\mathcal{P}_{\kappa}^{r}=\mathcal{P}_{0}^{r} \cap\left\{0 \leq \gamma^{\tau} \in \mathbb{R}^{i} \mid \gamma_{1} \geq \kappa\right\}$. Agora note que se $\bar{h}^{\text {cl }}$ é a solução do sistema reduzido $h_{0}^{c d}$, então $\bar{h}^{\text {cd }}(\kappa)$ dado por

$$
\begin{array}{rlr}
\dot{h}_{1}^{c}(\kappa) & =\bar{h}_{1}^{c}+\kappa, & \\
\bar{h}_{1}^{d}(\kappa) & =\bar{h}_{1}^{d}-\kappa=h_{1}-\left(h_{0}-1\right)-\kappa, & \\
\dot{h}_{j}^{c}(\kappa) & =\bar{h}_{j}^{c}, & \\
\bar{h}_{j}^{d}(\kappa) & =\bar{h}_{j}^{d}, & \text { para } 2 \leq j \leq 2 i-1 \\
\bar{h}_{2 i}^{c}(\kappa) & =h_{2 i}^{c}-\kappa=h_{2 i}-\left(h_{2 i+1}-1\right)-\kappa, & \\
\bar{h}_{2 i}^{d}(\kappa) & =\bar{h}_{2 i}^{d}+\kappa . &
\end{array}
$$

ć uma soluçāo inteira não negativa do sistema reduzido $h_{\kappa}^{c d}$, para inteiros $\kappa \mathrm{cm}\left[0, \min \left\{h_{1}-\right.\right.$ $\left.\left(h_{0}-1\right), h_{2 i}-\left(h_{2 i+1}-1\right)\right\}$ ]. Além disso, o vetor de números de Betti correspondente dado por (5.18) é o mesmo $\bar{\gamma}$. Portanto $\bar{\gamma}^{r} \in \mathcal{P}_{\kappa}^{r}$ para $\kappa$ em $\left[0, \min \left\{h_{1}-\left(h_{0}-1\right), h_{2 i}-\left(h_{2 i+1}-1\right)\right\}\right]$. Como $\mathcal{P}_{\kappa_{1}}^{r} \supset \mathcal{P}_{\kappa_{2}}^{r}$ se $\kappa_{1} \leq \kappa_{2}$, e $\gamma^{r}$ pertence a $\mathcal{P}_{\kappa}^{r}$, para todo $\kappa$ em $\left[0, \min \left\{h_{1}-\left(h_{0}-\right.\right.\right.$ 1), $\left.h_{2 i}-\left(h_{2 i+1}-1\right)\right\}$ ], concluímos que $\bar{\gamma}^{\tau}$ é o vetor máximo de $\mathcal{P}_{\kappa}^{r}$, para todo $\kappa \mathrm{cm}$ $\left[0, \min \left\{h_{1}-\left(h_{0}-1\right), h_{2 i}-\left(h_{2 i+1}-1\right)\right\}\right]$.

Os vários fatos estabelecidos até agora são ilustrados no próximo exemplo. Note que $\mathcal{P}_{\kappa}^{r}$ é também o casco convexo de $\mathcal{F}_{t} \cup \mathcal{F}_{0}$. Além disso, como $\mathcal{F}_{0}$ é a projeção de $\mathcal{F}_{t}$ e todos 
os elementos em $\mathcal{F}_{t}$ podem ser obtidos das soluções do sistema $h_{\kappa}^{c d}$ reduzido, temos meios, ainda que indiretos, de obter todos os elementos em $\mathcal{P}_{\kappa}^{r}$ dos vetores $h^{c d}$.

Exemplo Seja $n=2 i+1=7 \mathrm{e}\left(h_{0}, \ldots, h_{7}\right)=(1,5,11,10,5,3,4,3)$. A solução do sistema reduzido $h_{0}^{c d}$ que satisfaz $(5.16) \dot{c} \bar{h}^{c d}=(0,5,3,8,5,5,5,0,3,0,2,2)$, e as circulações elementares são $h^{\text {cd } 1}=(0,0,1,-1,0,0,0,0,-1,1,0,0)$ e $h^{\text {cd } 2}=$ $(0,0,0,0,1,-1,-1,1,0,0,0,0)$, correspondendo a ciclos 1 e 2 descritos na Figura 5.12. O elemento máximo de $\mathcal{P}_{0}$ é $\check{\gamma}=(1,2,3,5,5,3,2,1)$. Assim, a Proposição 5.3 .2 implica que politopo $\mathcal{P}_{\kappa}^{r}$ é dado pelas desigualdades

$$
\begin{aligned}
\gamma_{1} & \leq 2 \\
\gamma_{1}-\gamma_{2} & \geq-1 \\
\gamma_{1}-\gamma_{2}+\gamma_{3} & \leq 4 \\
\gamma_{1} & \geq \kappa \\
\gamma_{1}, \gamma_{2}, \gamma_{3} & \geq 0
\end{aligned}
$$

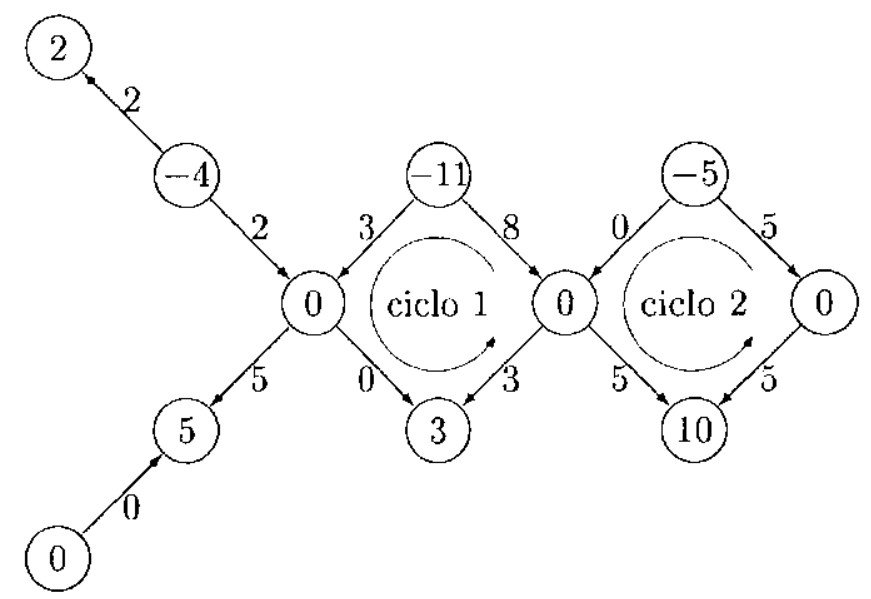

Figura 5.12: Solução $\tilde{h}^{\text {cd }}$ do exemplo.

Neste caso $\kappa$ pode assumir os valores 0,1 e 2. A Figura 5.13 descreve os três politopos, delineando suas arestas e enfatizando seus elementos inteiros. A relação entre dois $\gamma^{r}$ s em $\mathcal{F}_{t}$ e seus $h^{c d}$ 's correspondentes pode ser obtida pela base na seguinte tabela. 


\begin{tabular}{c|c}
$\hat{\gamma}^{r}-\tilde{\gamma}^{r}$ & $\hat{h}^{c d}-\tilde{h}^{c d}$ \\
\hline$(-1,-1,0)$ & $h^{c d 1}$ \\
$(0,-1,-1)$ & $h^{c d 2}$
\end{tabular}

Assim a solução do sistema reduzido $h_{0}^{\text {cd }}$ correspondente a $\hat{\gamma}^{r}=(1,2,5)=\bar{\gamma}^{r}+(-1,-1,0)$ é $h^{c d}=\bar{h}^{c d}+h^{c d 1}=(0,5,4,7,5,5,5,0,2,1,2,2)$. Da mesma forma, se $\hat{\gamma}^{r}=(1,1,4)=$ $\bar{\gamma}^{r}+(-1,-2,-1)=\bar{\gamma}^{r}+(-1,-1,0)+(0,-1,-1)$, então $h^{c d}=\bar{h}^{c d}+h^{c d 1}+h^{c d 2}=$ $\left(0,5,4,7,6,4,4, \lambda_{3}, 2,1,2,2\right)$.

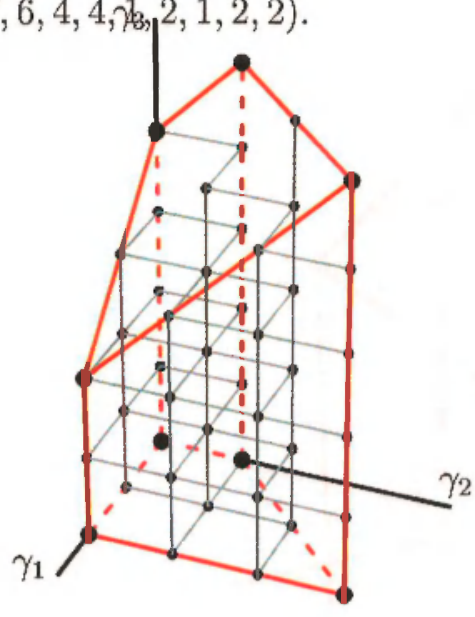

(a) $\mathcal{P}_{0}^{r}$ e seus elementos inteiros.

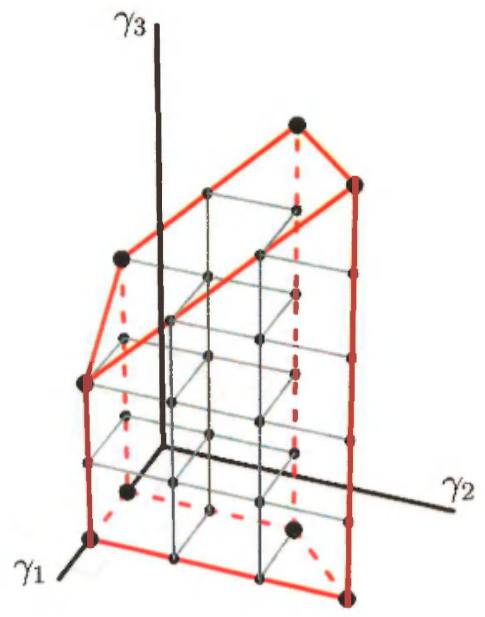

(b) $\mathcal{P}_{1}^{r}$ e seus elementos inteiros.

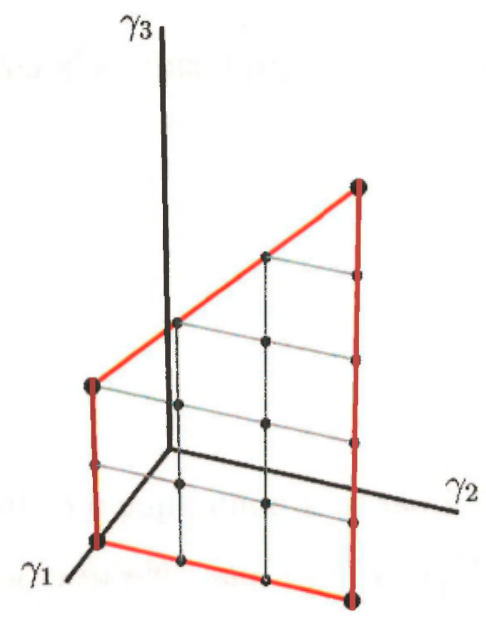

(c) $\mathcal{P}_{2}^{r}$ e seus elementos inteiros.

Figura 5.13: Politopos $\mathcal{P}_{\kappa}^{r}$. 
Apenas como curiosidade mostramos na Figura 5.14 todos os $\gamma^{r}$ 's inteiros que são gerados de soluções inteiras, não necessariamente não negativas, para o sistema reduzido $h_{\kappa}^{c d}$. Como o fluxo no ciclo 1 pode variar de 0 a 3 e no ciclo 2 de 0 a 5 , existem 24 fluxos inteiros não negativos que resolvem o sistema $h_{\kappa}^{c d}$. Como esperado, os $\gamma^{r}$ 's correspondentes satisfazem as desigualdades de Morse (de fato, eles pertencem a $\left\{\gamma^{r} \in \mathbb{R}^{3} \mid \gamma_{1}-\gamma_{2}+\gamma_{3}=\right.$ 4\}), mas não são necessariamente não negativos.

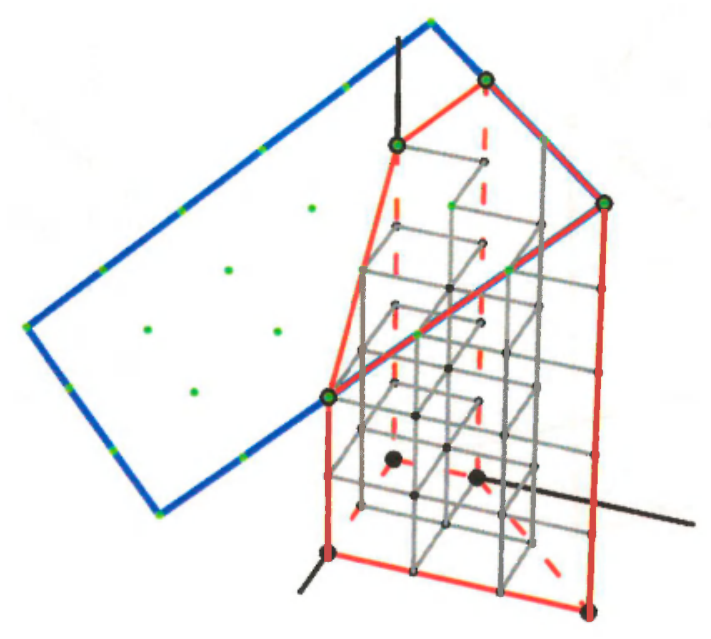

Figura 5.14: Face estendida $\mathcal{F}_{t}$ produzida pela circulação.

\subsubsection{Caso $n$ par}

Inicialmente, seja $n=2 i$, onde $i \geq 2$ é par, e suponha que o conjunto de dados de índice pré-fixado $\left(h_{0}, \ldots, h_{2 i}\right)$ é tal que $\sum_{j=0}^{i i}(-1)^{j} h_{j}$ è par. Neste caso temos as condições de dualidade para eliminar $\gamma_{i+1}, \ldots, \gamma_{2 i-1}$, as condições de fronteira para eliminar $\gamma_{0}$ e $\gamma_{2 i}$, e a primeira equação em (1) para eliminar $\gamma_{\imath}$ do sistema de desigualdades que define $\mathcal{P}_{\kappa}$. Como antes, o sistema resultante de desigualdades $(5.28)$ em $\left(\gamma_{1}, \ldots, \gamma_{i-1}\right)$ define um politopo $\mathcal{P}_{\kappa}^{r}$ cujos elementos estão em correspondência 1-â-1 com os elementos de $\mathcal{P}_{\kappa}$. 
Detalhes da dedução de (5.28) são provados no Capítulo 4.

$$
\begin{aligned}
& 0 \geq 1-h_{2 i} \\
& \sum_{j=1}^{k}(-1)^{j+1} \gamma_{j}\left\{\begin{array}{l}
\leq 1+\sum_{j=2 i \cdot k}^{2 i}(-1)^{j+1} h_{j}, \text { se } 1 \leq k \leq i-1, k \text { ímpar } \\
\geq 1+\sum_{j=2 i-k}^{2 i}(-1)^{j+1} h_{j}, \text { se } 1 \leq k \leq i-1, k \text { par }
\end{array}\right. \\
& (-1)^{i} \sum_{j-1}^{i-1}(-1)^{j+1} \gamma_{j} \geq(-1)^{i}\left(1+\frac{1}{2} \sum_{j=0}^{2 \imath}(-1)^{j+1} h_{j}\right) \\
& \sum_{j=1}^{2 i-k}(-1)^{j+1} \gamma_{j}\left\{\begin{array}{l}
\leq 1+\sum_{j-0}^{2 i-k}(-1)^{j+1} h_{j}, \quad \text { se } i+1 \leq k \leq 2 i-1, k \text { ímpar } \\
\geq 1+\sum_{j=0}^{2 i-k}(-1)^{j i 1} h_{j}, \quad \text { se } i+1 \leq k<2 i-1, k \text { par }
\end{array}\right. \\
& 0 \geq 1-h_{0} \\
& \gamma_{j} \geq 0, \quad \text { para } 1 \leq j \leq i-1 \\
& \gamma_{1} \geq \kappa \text {. }
\end{aligned}
$$

A seguinte proposiçāo foi provada no Capítulo 4 para o caso $\kappa=0$.

Proposição 5.3.3 O politopo $\mathcal{P}_{\kappa}^{r}$ definido por (5.28) tem vértices inteiros e cada $\gamma^{r}$ (inteiro) no politopo corresponde a um $h^{\text {cd }}$ (inteiro) näo negativo satisfazendo (5.15). Cada vértice de $\mathcal{P}_{\kappa}^{r}$ pertence a uma das três faces:

$$
\begin{aligned}
& \mathcal{F}_{t}=\left\{\gamma \in \mathcal{P}_{\kappa}^{r} \mid \sum_{j=1}^{i-1}(-1)^{j+1} \gamma_{j}=1+\min \left\{\sum_{j=i+1}^{2 i}(-1)^{j+1} h_{j}, \sum_{j=i+1}^{2 i}(-1)^{j+1} h_{j}\right\}\right\} \\
& \mathcal{F}_{b}=\left\{\gamma \in \mathcal{P}_{\kappa}^{r}: \sum_{j=1}^{i-1}(-1)^{j+1} \gamma_{j}=1+\frac{1}{2} \sum_{j=0}^{2 i}(-1)^{j+3} h_{j}\right\} \\
& \mathcal{F}_{0}=\left\{\gamma^{r} \in \mathcal{P}_{\kappa}^{r} \mid \gamma_{i-1}=0\right\} .
\end{aligned}
$$

Demonstração: A prova no Capítulo 4 pode ser facilmente adaptada ao caso $\kappa \neq 0$, como feito para Proposição 5.3.1.

As desigualdades definindo $\mathcal{P}_{\kappa}^{r}$ podem ser bem simplificadas se recorrcmos ao uso de $\bar{\gamma}$, o vetor dos números de Betti em $\mathcal{P}_{\kappa}$ correspondendo a solução $\bar{h}^{c d}$ do sistema reduzido $h_{\kappa}^{c d}$ 


\section{5. Grafos de Lyapunov, Desigualdades de Poincaré-Hopf e Desigualdades de Morse}

que satisfaz as condiçōes de complementaridade (5.16). A próxima proposição é análoga à Proposição 5.3.2 para o caso $n=0 \bmod 4$.

Proposição 5.3.4 Suponha que (5.15) tem uma solução não negativa. O politopo $\mathcal{P}_{\kappa}^{r}$ pode visto como

$$
\mathcal{P}_{\kappa}^{r}=\left\{0 \leq \gamma^{r} \mid \begin{array}{rl}
(-1)^{k+1} \sum_{j=0}^{k}(-1)^{j+1} \gamma_{j} & \leq(-1)^{k+1} \sum_{j=0}^{k}(-1)^{j+1} \ddot{\gamma}_{j}, \quad \text { para } 1 \leq k \leq i-1 \\
\sum_{j=0}^{i=1}(-1)^{j+1} \gamma_{j} & \geq \frac{1}{2} \sum_{j=0}^{2 i}(-1)^{j+1} h_{j} \\
\gamma_{1} & \geq \kappa
\end{array}\right\}
$$

Além disso, $\bar{\gamma}^{r}$ é o vetor máximo em $\mathcal{P}_{\kappa}^{r}$, componente a componente.

Demonstração: A adaptação da prova da proposição correspondente no Capítulo 4 imita o que foi feito na prova da Proposição 5.3.2.

Desenvolvimentos análogos, isto é, redução no número de variáveis e versōes das Proposições 5.3 .3 e 5.3 .4 podem ser obtidas para os casos $n=2 \bmod 4$ diretamente.

Concluímos esta seção com a observação que a família $\mathcal{L}\left(h_{0}, \ldots, h_{n}, \kappa\right)$ está associada ao $\mathcal{P}_{\kappa}^{r}$. Esta associação é estabclecida pelo fato que qualquer grafo $\mathrm{cm} \mathcal{L}\left(h_{0}, \ldots, h_{n}, \kappa\right)$ tem um conjunto de dados fixados que determinam $\mathcal{P}_{\kappa}^{r}$. Embora todo grafo em $\mathcal{L}\left(h_{0}, \ldots, h_{n}, \kappa\right)$ satisfaça as desigualdades de Morse para qualquer vetor de números de Betti em $\mathcal{P}_{\kappa}^{r}$ é um problema aberto se estes grafos podem ser realizados como fluxos sobre variedades com estes números de Betti.

O que sabemos é que para uma dada continuação, $L\left(h_{0}, h_{1}^{c}, h_{1}^{d}, \ldots, h_{n-1}^{c}, h_{n-1}^{d}, h_{n}, \kappa\right)$, do grafo $L\left(h_{0}, h_{1}, \ldots, h_{n-1}, h_{n}, \kappa\right)$ existe uma correspondência com um elemento inteiro $v$ sobre a face estendida $\mathcal{F}_{t}$ de $\mathcal{P}\left(h_{0}, \ldots, h_{n}\right)$ como na Figura 5.14. De fato, este elemento em $L\left(h_{0}, h_{1}^{c}, h_{1}^{d}, \ldots, h_{n-1}^{c}, h_{n-1}^{d}, h_{n}, \kappa\right)$ que define as coordenadas do vetor dos números de Betti que determinam $v$. Também, nāo existe interpretação topológica para os grafos $L\left(h_{0}, h_{1}^{c}, h_{1}^{d}, \ldots, h_{n-1}^{c}, h_{n-1}^{d}, h_{n}, \kappa\right)$ que determinam vetores de números de Betti fora do quadrante não negativo. 


\section{Lista de Figuras}

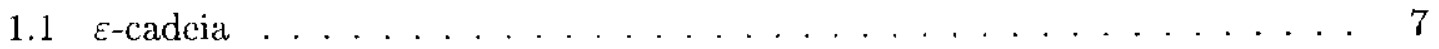

1.2 Função de Lyapunov. . . . . . . . . . . . . . . . . . . . . . . . . . . . . . . 8

1.3 Grafo de Lyapunov. . . . . . . . . . . . . . . . . . . . . . . . . . 9

1.4 Grafo abstrato de Lyapunov em dimensão $7 \ldots$. . . . . . . . . . . . . 10

1.5 Conjunto invariante isolado. . . . . . . . . . . . . . 12

1.6 Par-índice. . . . . . . . . . . . . . . . . . . . 12

1.7 Par-Índice . . . . . . . . . . . . . . . . . . . 13

1.8 Blocos isolantes para selas em dimensão $2 \ldots \ldots \ldots \ldots$

1.9 Blocos isolantes para selas em dimensäo $3 \ldots \ldots \ldots$

1.10 Passos para obtenção de $\mathcal{H}(S) \ldots \ldots \ldots \ldots \ldots$

1.11 Passos para obtençāo de $\mathcal{H}(S) \ldots \ldots \ldots \ldots \ldots \ldots$

1.12 Passos para obtenção de $\mathcal{H}(S) \ldots \ldots \ldots \ldots \ldots \ldots \ldots$

1.13 Passos para obtenção de $\mathcal{H}(S) . \ldots \ldots \ldots \ldots \ldots$

1.14 Continuaçāo do índice de Conley. . . . . . . . . . . . . . . . 16

$1.15 \Lambda$ lça $H$ a ser colada. . . . . . . . . . . . . . . . . 17

1.16 Colando alças em dimensão $2 \ldots \ldots \ldots \ldots \ldots \ldots$

1.17 Os três possiveis efeitos algébricos. . . . . . . . . . . . . 18

1.18 Semi-grafos de Lyapunov para selas em dimensão $2 \ldots \ldots$

1.19 Semi-grafos de Lyapunov para selas em dimiensão $3 \ldots \ldots$. . . . . . . . . . 19

1.20 Condições locais para um grafo de Lyapunov do tipo Morse. . . . . . . . 20

1.21 Vértice a ser explodido. . . . . . . . . . . . . . . . . 23

1.22 Explosões do vértice . . . . . . . . . . . . . . . . . 24

3.1 Vértice do tipo sela generalizada. . . . . . . . . . . . . 41 
3.2 Explosão partial de um vértice do tipo sela generalizada. . . . . . . . . . 42

3.3 Vértice Repulsor. . . . . . . . . . . . . . . . . . . . . . 43

3.4 Explosāo parcial de um vértice repulsor. . . . . . . . . . . . . . 43

3.5 Vértice a ser explodido. . . . . . . . . . . . . . . . . 45

3.6 Explosāo das arestas incidentes negativamente. . . . . . . . . . . . . 46

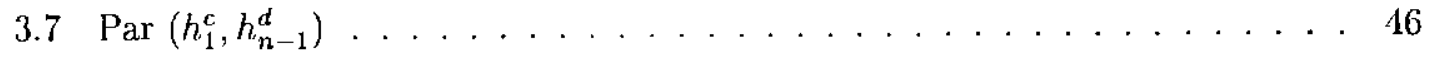

3.8 Arestas incidentes negativamente explodidas e ciclos inseridos. . . . . . . 47

3.9 Explosão linear: $L$ e $L^{\dagger} \ldots \ldots \ldots \ldots \ldots$

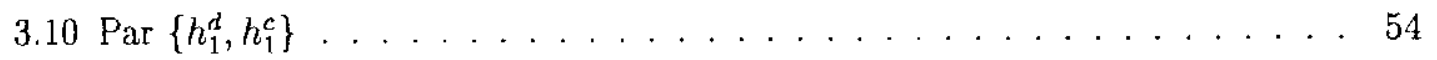

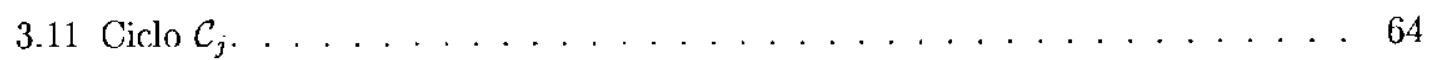

3.12 Estrutura de digrafo $\mathcal{G} \ldots \ldots \ldots \ldots 7$

3.13 O subgrafo $\mathcal{G}_{j}$ consiste dos ciclos não-orientados $\mathcal{C}_{j}$, para $j=1, \ldots, i-2 . \quad 84$

3.14 o subgrafo $\mathcal{G}_{i-1} \ldots \ldots \ldots \ldots \ldots \ldots$

3.15 Estrutura do digrafo $\mathcal{G} \ldots \ldots \ldots \ldots \ldots$

4.1 Examplo de rede, para $i=3 \ldots \ldots \ldots$. . . . . . . . . . . . . . . . .

4.2 Soluçāo $\tilde{h}^{\text {cd }}$ do exemplo. . . . . . . . . . . . . . . . . . 127

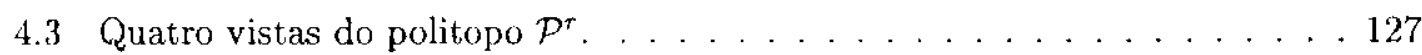

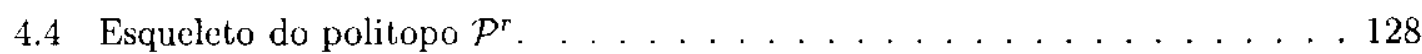

4.5 Solução $\tilde{h}^{\text {cd }}$ do exemplo. . . . . . . . . . . . . . . . . . 134

4.6 Faces de $\mathcal{P}^{r}$ e grade inteira. . . . . . . . . . . . . . 136

5.1 Rede para o caso $n=2 i+1, i$ ímpar. . . . . . . . . . . 141

$5.2 \quad$ Rede para $n=2 i+1, i$ par. . . . . . . . . . . . . 141

5.3 Rede para o caso $n=2 i=0 \bmod 4 \ldots \ldots \ldots 143$

5.4 Rede para o caso $n=2 i=2 \bmod 4 \ldots \ldots \ldots . \ldots \ldots$

5.5 Dividindo o problema em dois. . . . . . . . . . . . . . . . . 145

5.6 Divisão seguinte. . . . . . . . . . . . . . . . . . 146

5.7 Hltimos problemas independentes. . . . . . . . . . . . . . . . 147

5.8 Exemplo genérico de ciclo de rede. . . . . . . . . . . . . . . . 149

5.9 Grafo abstrato de Lyapunov $L(2,4,1,2,1,1,4,1, \kappa=2) \ldots \ldots \ldots$

5.10 Grafos na família $\mathcal{L}(2,4,1,2,1,1,4,1, \kappa=2) \ldots \ldots \ldots \ldots$ 
5.11 Implosão de $L(2,4,1,2,1,1,4,1, \kappa=2) \ldots \ldots \ldots \ldots \ldots$

5.12 Solução $\bar{h}^{c d}$ do exemplo. . . . . . . . . . . . . . . . . 164

5.13 Politopos $\mathcal{P}_{\kappa}^{r} \ldots \ldots \ldots \ldots \ldots$. . . . . . . . . . . . . . . . . . . .

5.14 Face estendida $\mathcal{F}_{t}$ produzida pela circulação. . . . . . . . . . . . 166 


\section{Tabela de Símbolos}

\section{Ordem alfabética}

$B_{j}^{+} \quad$ soma dos $j$-ćsimos números de Betti das arestas incidentes positivamente

$B_{j}^{-} \quad$ soma dos $j$-ćsimos números de Betti das arestas incidentes negativamente

$D^{j} \quad j$-disco unitário fechado

$e^{+} \quad$ aresta incidente positivamente

$e^{-} \quad$ aresta incidente negativamente

$f \quad$ função de Lyapunov

$g(M) \quad$ gênero de Comea

$H \quad$ handle $D^{\ell} \times D^{n-\ell}$

$h_{j} \quad$ dimensão do índice homológico de Conley

$\breve{h}_{j} \quad$ dimensāo do índice homológico de Conley para o fluxo reverso

$\mathcal{H}(S) \quad$ índice de Conley

$\mathrm{CH}_{*}(\mathrm{~S}) \quad$ índice homológico de Conely

I intervalo

$J \quad$ intervalo aberto

L $\quad$ grafo de Lyapunov

$L\left(h_{0}, \ldots, h_{n}, \kappa\right)$ grafo abstrato de Lyapunov

$L_{B} \quad$ semi-grafo

$\mathcal{L}\left(h_{0}, \ldots, h_{n}, \kappa\right)$ família de grafos de Lyapunov

$M \quad$ variedade orientável fechada de dimensão $n$

$N \quad$ bloco isolante

$\mathrm{N}^{+} \quad$ conjunto de entrada para o fluxo

$\mathrm{N}^{-} \quad$ conjunto de saída para o fluxo 


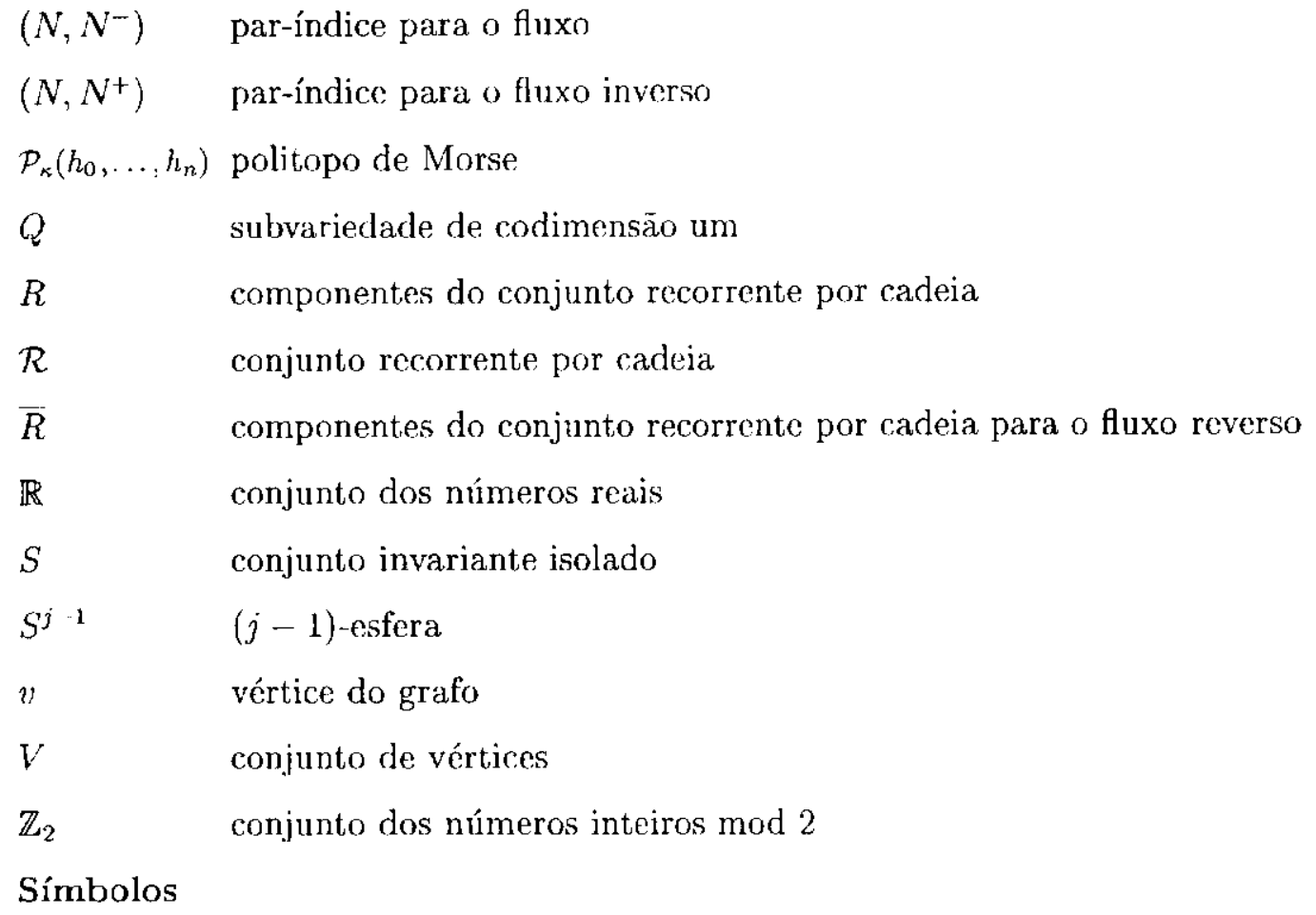

Símbolos

$\sim \quad$ relação de equivalência

\section{Letras gregas}

$\begin{array}{ll}\phi_{t} & \text { fluxo contínuo Phi } \\ \phi_{-t} & \text { fluxo reverso } \\ \kappa & \text { número de ciclos } \\ \kappa_{v} & \text { número de ciclos do vértice } \\ \kappa_{L} & \text { cycle rank } \\ \gamma & \text { número de Betti de } M \\ \beta & \text { número de Betti das arestas do grafo }\end{array}$




\section{Referências Bibliográficas}

[1] A. Bachem and M. Grötschel. New aspects of polyhedral theory. In: B. Korte, ed., Applied Modern Mathematics. North-Holland, Amsterdam, 1982.

[2] M. A. Bertolim, M. P. Mello and K. A. de Rezende. Lyapunov graph continuation. Technical Report IMECC-UNICAMP, RP46/01. Available in //www.ime.unicamp.br/rel.pesq/2001/rp46-01.html. To appear in Ergodic Theory Dynam. Systems.

[3] M. A. Bertolim, M. P. Mello and K. A. de Rezende. Poincaré-Hopf Inequalities. Technical Report IMECC-UNICAMP, RP 19-02. Available in //www.ime.unicamp.br/rel pesq/2002/rp19-02.html.

47] M. A. Bertolim, M. P. Mello and K. A. de Rezende. Lyapunov Graphs, PoincaréHopf and Morse Inequalities. Technical Report IMECC-UNICAMP, RP 40-02. Available in //www.ime.unicamp.br/rel pesq/2002/rp40-02.html. Subrnitted to Annals of Mathematics.

[5] C. Conley. Isolated invariant sets and the Morse index. CBMS Regional Conference Series in Mathematics, 38. Amcrican Mathematical Society, Providence, R.I., 1978.

[6] O. Cornea. The genus and the fundamental group of high-dimensional manifolds. Stud. Cerc. Mat. 41 (1989), no. 3, 169-178.

[7] R. N. Cruz and K. A. de Rezende. Gradient-like flows on high-dimensional manifolds. Ergodic Theory Dynam. Systems 19 (1999), no. 2, 339-362. 
[8] R. N. Cruz and K. A. de Rezende. Cycle rank of Lyapunov graphs and the genera of manifolds. Proceedings of the American Mathematical Society 126 (1998), no. $12,3715-3720$.

[9] K. A. de Rezende. Smale flows on the three-sphere. Transactions of the American Mathematical Society 303 (1987), no. 1, 283-310.

[10] K. A. de Rezende. Gradient-like flows on 3-manifolds. Ergodic Theory Dynam. Systems 13 (1993), no. 3, 557-580.

[11] K. A. de Rezende and R. D. Franzosa. Lyapunov graphs and flows on surfaces. Trans. Amer. Math. Soc. 340 (1993), no. 2, 767-784.

[12] J. Franks. Nonsingular Smale flows on $S^{3}$. Topology 24 (1985), no. 3, 265-282.

[13] D. R. Fulkerson and O. A. Gross. Incidence matrices and interval graphs. Pacific Journal of Mathematics 15 (1965), 835-855.

[14] F. Granot and A. F. Veinott, Jr. Substitutes, complements and ripples in network flows. Mathematics of Operations Research, 10(3), August 1985.

[15] A. J. Hoffman and J. B. Kruskal. Integral boundary points of convex polyhedra. In Linear Inequalities and Related Systems (H. W. Kuhn and A. W. Tucker, eds.), 223-246, Annals of Mathematics Studies, No. 38, Princeton University Press, Princeton, N.J., 1956.

[16] C. McCord. Poincaré-Lefschetz duality for the homology Conley index. Trans. Amer. Math. Soc. 329 (1992), no. 1, 233-252.

[17] J. Milnor. Morse Theory. Based on lecture notes by $M$. Spivak and R. Wells. Annals of Mathematics Studies, No. 51 Princenton Lniversity Press, Princeton, N. J. 1963.

[18] H. Poincaré. Second complément à l'analysis situs Proceedings of the London Mathematical Society, 32 (1900) 277-308 ireprinted in Ouvres. de Henri Poincaré, Tome VI. Gauthier-Villars, Paris, 1953, pp.338-370.] 
[19] J. F. Reincck. Continuation to Cradient Flows. Duke Mathematical Journal 64 (1991), no. 2, 261-269.

[20] R. T. Rockafellar. The elementary vectors of a subspace of $\mathbb{R}^{n}$. In R. C. Bose and T. A. Dowling, editors, Combinatorial Mathematics and its Applications, pages 104-127. University of North Carolina Press, 1969.

[21] A. Schrijver. Theory of Lincar and Integer Programming. Wiley, Chichester, 1986.

[22] W. T. Tutte Connectvity in Graphs. Univ. Toronto Press, Toronto, 1966. 


\section{Índice Remissivo}

$(\ell-1)$-conectante, 18

$\beta$-invariante, 18

$\ell$-desconcctante, 17

algoritmo

da explosão, 40

da explosão para $n=2,53$

aresta

direcionada, 11

do grafo, 9

semi-aresta, 11

Betti

vetor de números de, 2,10

bloco isolante, 12

ciclo elementar, 46

conjunto

invariante, 11

isolado. 11

recorrente por cadeia, 7

Conley

continuação do índice de, 15

dualidade do índice de, 29

Teorema de, 8

continuação, 15

de um grafo, 23 do índice de Conley, 15

Teorema de, 39

prova, 95

Cornea

gênero de, 2

cycle rank, 2

desigualdades de Morse duais, j

dualidade do índice de Conley, 29

explosão de um vértice, 22

fluxo

do tipo gradiente, 8

função de Lyapunov, 1, 8

grafo

continuação de um, 23

de Lyapunov, 1, 9

implosão, 23

semi-grafo direcionado, 11

grafo abstrato

de Lyapunov do tipo Morse, 20

de Iyapunov em dimensão $n, 1,9$

gènero de Cornea, 2

implosĩo do grafo $L, 23$

Lyapunov 
função de, 1,8

grafo abstrato de, 9

grafo abstrato de Lyapunov do tipo

Morse, 20

grafo abstrato em dimensão $n, 1$

grafo de, 1, 9

Morse

desigualdades duais, 5

grafo abstrato de Lyapunov do tipo,

20

politopo de, 4

par-índice, 11

politopo de Morse, 4

ponto recorrente por cadeia, 7

semi-arestas, 11

semi-grafo direcionado, 11

sistema $h_{\kappa}^{c d}, 51$

Sistemas Lineares

soluções, 54

soluções para os Sistemas Lineares, 54

\section{Teorema}

de Conley, 8

de Continuação, 39

prova, 95

de equivalência, 99

teoria de alças, 16

totalmente unimodular, 56

vetor de números de Betti, 2, 10

vizinhança isolante, 11

vértice

atrator, 41

do grafo, 9

do tipo sela, 41

generalizada, 41

repulsor, 41 